University of Louisville

ThinkIR: The University of Louisville's Institutional Repository

Electronic Theses and Dissertations

$12-2011$

\title{
Metal organic chemical vapor deposition of InGaN alloys on nanowire substrates.
}

Chandrashekhar Pendyala

University of Louisville

Follow this and additional works at: https://ir.library.louisville.edu/etd

\section{Recommended Citation}

Pendyala, Chandrashekhar, "Metal organic chemical vapor deposition of InGaN alloys on nanowire substrates." (2011). Electronic Theses and Dissertations. Paper 1111.

https://doi.org/10.18297/etd/1111

This Doctoral Dissertation is brought to you for free and open access by ThinkIR: The University of Louisville's Institutional Repository. It has been accepted for inclusion in Electronic Theses and Dissertations by an authorized administrator of ThinkIR: The University of Louisville's Institutional Repository. This title appears here courtesy of the author, who has retained all other copyrights. For more information, please contact thinkir@louisville.edu. 


\title{
METAL ORGANIC CHEMICAL VAPOR DEPOSITION OF InGaN ALLOYS ON NANOWIRE SUBSTRATES
}

\author{
By \\ Chandrashekhar Pendyala \\ Master of Science, Cleveland State University, 2006
}

\begin{abstract}
A Dissertation
Submitted to the Faculty of the

J. B. Speed School of Engineering, University of Louisville

in Partial fulfillment of the Requirements

for the Degree of

Doctor of Philosophy

Department of Chemical Engineering

University of Louisville

Louisville, KY 40292
\end{abstract}

December 2011 
Copyright 2011 by Chandrashekhar Pendyala

All rights reserved 


\title{
METAL ORGANIC CHEMICAL VAPOR DEPOSITION OF InGaN ALLOYS ON NANOWIRE SUBSTRATES
}

\author{
By \\ Chandrashekhar Pendyala \\ Master of Science, Cleveland State University, 2006
}

A Dissertation Approved on

November 7, 2011

By the Following Dissertation Committee

Dr. Mahendra K. Sunkara (Dissertation Director)

\begin{tabular}{c}
\hline Dr. Thomas Starr \\
\hline Dr. Gamini U. Sumanasekera \\
\hline Dr. Moises Carreon \\
\hline
\end{tabular}

Dr. Bruce W. Alphenaar

Dr. Madhu Menon 


\section{DEDICATION}

This Dissertation is dedicated to my parents and sister Visala Pendyala, Venkata Ramana Murthy Pendyala and Kavita Pendyala 


\section{ACKNOWLEDGEMENTS}

First of all, I would like to thank Dr. Mahendra Sunkara for giving me an opportunity to work in his group and for this constant guidance and support. I thoroughly enjoyed my work with the excellent synthesis and characterization facilities in the lab.

I would also like to thank Dr. Sumanasekera, Dr. Starr, Dr. Carreon, Dr.

Alphenaar and Dr. Menon for serving on my thesis committee and for their valuable suggestions.

I would also like to thank the past and current members of my group. I had an enjoyable experience working with them. I would like to thank Jeong for designing the MOCVD reactor and for helping me become more hands on. I would also like to thank Dr. Hari Chandrasekaran, Dr. Sreeram Vaddiraju and Dr. Hongwei Li for helping me in nanowire growth studies.

Finally, I would like to thank my parents and sister for their constant encouragement throughout. 


\begin{abstract}
METAL ORGANIC CHEMICAL VAPOR DEPOSITION OF InGaN ALLOYS ON NANOWIRE SUBSTRATES
\end{abstract}

\author{
Chandrashekhar Pendyala
}

November 2011

Rising environmental concerns due to our rising population ad energy demand along with our excessive dependence on fossil fuels has created an urgent need to find clean, renewable and carbon free source of energy. Photoelectrochemical (PEC) water splitting is a clean and carbon free process where hydrogen is produced from water and sunlight using a semiconductor. To date, no material has been found that meets the stringent requirements of band gap, band edge positions and stability for spontaneous water splitting. It is however possible to use two materials to meet the criteria. In this regard, InGaN alloys with indium rich composition are interesting materials. However, very little is understood about the synthesis of thick $(\sim 200-300 \mathrm{~nm})$, single crystal InGaN layers for PEC applications.

Heteroepitaxial growth of InGaN films on planar substrates induces phase segregation due to stress. Here, we proposed to investigate the role of nanowires as strain relaxing substrates to mitigate phase segregation. GaN nanowires with controlled 
orientation and small diameters were synthesized on various substrates by controlling the temperature and material flux to control the nuclei formation. The mechanism to control the growth mode using equilibrium solubility was validated with the III-Sb system.

InGaN layers with controlled composition were synthesized on the GaN nanowires in a custom built MOCVD reactor. The InGaN layers are single crystalline, without any phase segregation. It was observed that only nanowires with diameters $<30$ $\mathrm{nm}$ led to the observation while nanowires with larger diameters $(\sim 100 \mathrm{~nm})$ act as planar substrates resulting in polycrystalline growth. The heteroepitaxial growth was observed to evolve from initial InGaN islands coalescing into single crystalline shell on the GaN nanowires. Morphology of the InGaN shells was observed to depend on the orientation of the $\mathrm{GaN}$ nanowire substrates with $\mathrm{c}-\mathrm{GaN}$ nanowires resulting in hexagonal shell while a-GaN nanowires had rectangular shell.

We also investigated a novel material system GaSbN using theoretical techniques for its applicability toward PEC water splitting. The electronic structure of GaSbN system with dilute antimony was investigated using theoretical simulations. Results indicate that only very small antimony content $(<10 \%)$ is required to achieve the right band gap. Most importantly, the band edges of GaSbN alloy seem to straddle the water splitting potentials that makes it a potential direct water splitting material. 
TABLE OF CONTENTS

PAGE

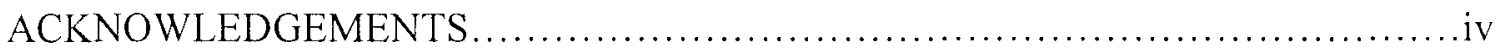

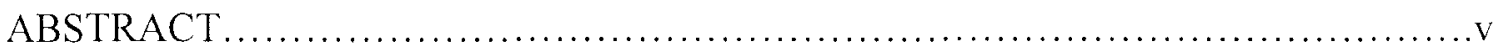

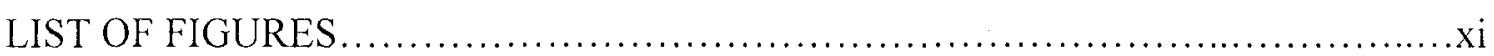

CHAPTER

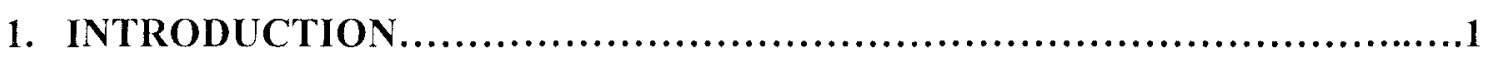

1.1 ENERGY AND ENVIRONMENTAL CHALLENGES ..............................

1.2 ISSUES WITH HYDROGEN PRODUCTION ........................................

1.3 PHOTOELECTROCHEMICAL WATER SPLITTING................................................

1.3.1 PEC Water Splitting: Basics ..........................................................

1.3.2 PEC Water Splitting: Challenges.................................................

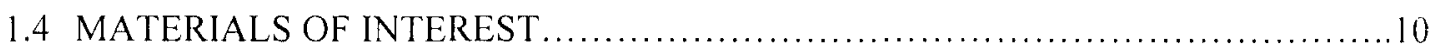

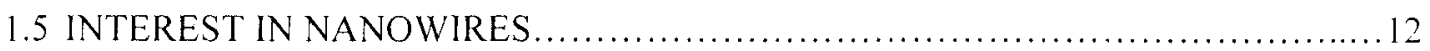

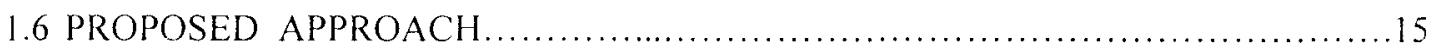

1.7 ORGANIZATION OF THESIS .....................................................

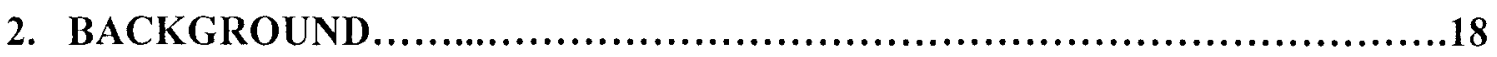

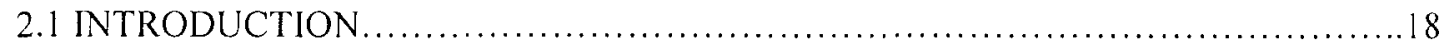

2.2 REVIEW OF PEC WATER SPLITTING MATERIALS..............................18

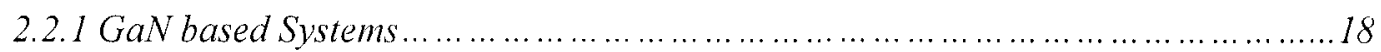




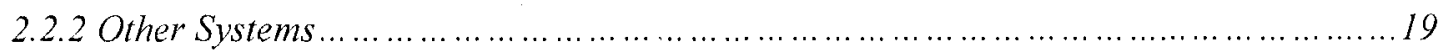

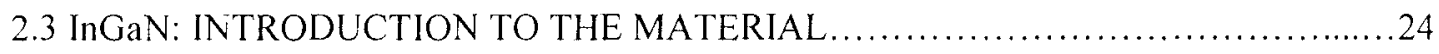

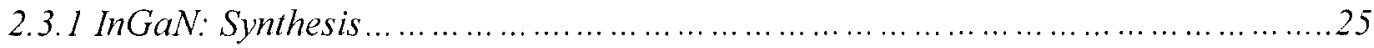

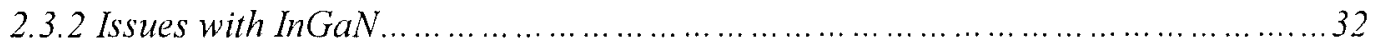

2.4 REVIEW OF HETEROEPITAXIAL GROWTH OF InGaN $\ldots \ldots \ldots \ldots \ldots \ldots \ldots \ldots \ldots \ldots$

2.5 HETEROEPITAXIAL GROWTH ON NANOWIRE SUBSTRATES .................46

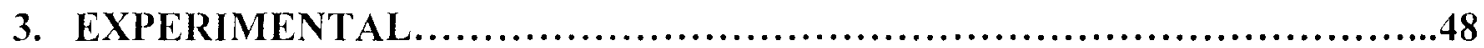

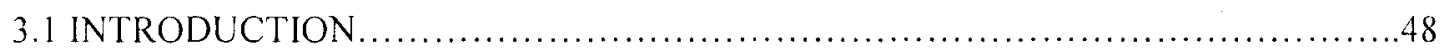

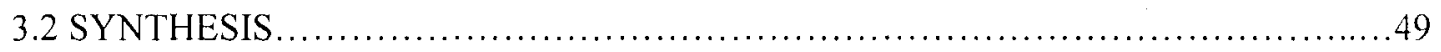

3.2.1 Reactive vapor transport reactor configurations ................................. 49

3.2.2 Metal Organic Chemical Vapor Deposition reactor ..................................52

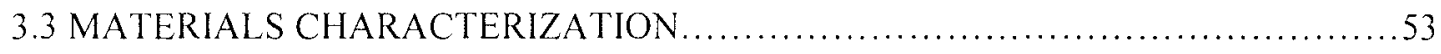

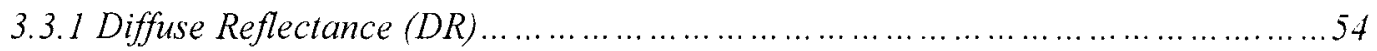

3.3.2 Selected Area Electron Diffraction (SAED) .................................... 55

3.3.3 Convergent Beam Electron Diffraction (CBED) ...................................57

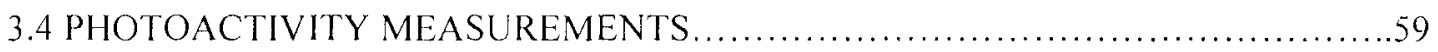

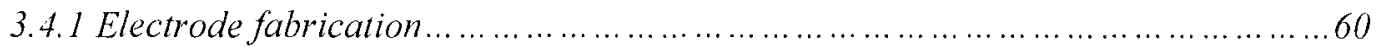

3.4.2 Illuminated Open Circuit Potential measurement ...................................6 60

3.4.3 Photocurrent measurement ...................................................... 61

4. MOCVD REACTOR DESIGN AND MODELING...........................63

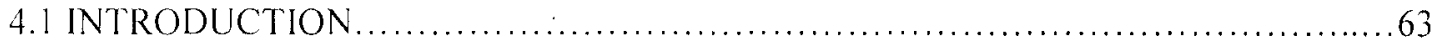

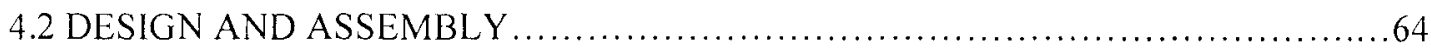

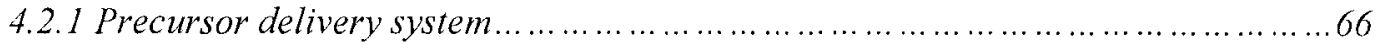

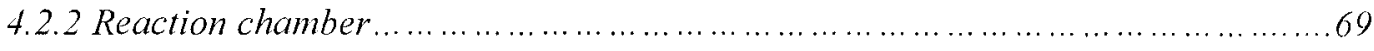

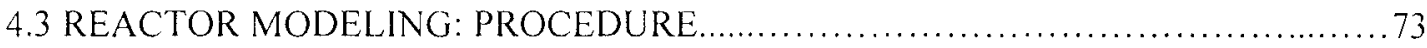

viii 


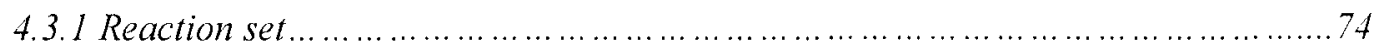

4.3.2 Modeling procedure ...................................................... 76

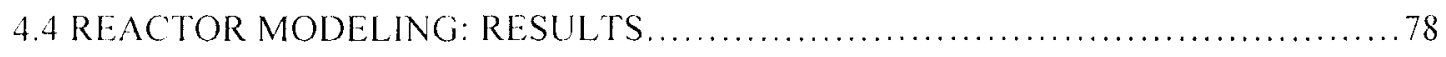

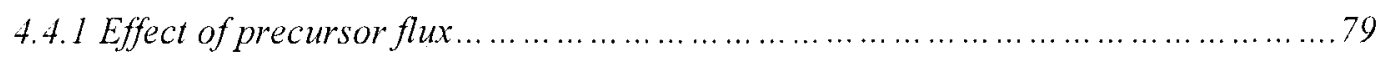

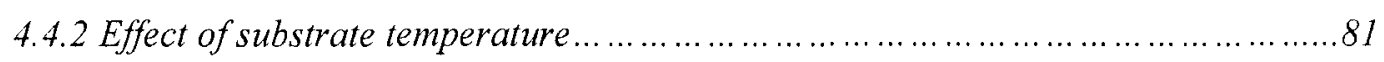

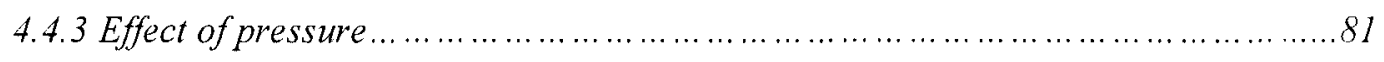

4.4.4 Effect of separation of the substrate and showerhead .......................... 83

5. SYNTHESIS OF GaN AND III-ANTIMONIDE NANOWIRES...............89

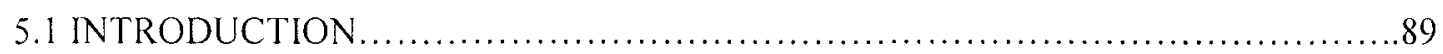

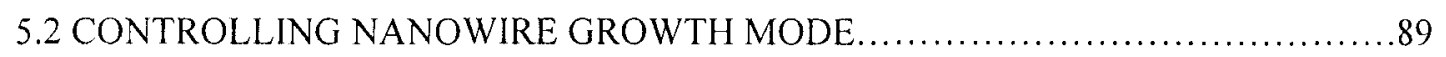

5.2.1 Low melting metal mediated nanowire growth ................................. 9 I

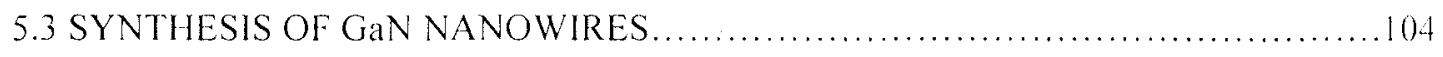

5.3.I Self-catalyzed synthesis of GaN nanowires................................. 104

5.3.2 Copper catalyzed synthesis of GaN nanowires.................................... 109

6. SYNTHESIS OF InGaN ON GaN NANOWIRES...........................114

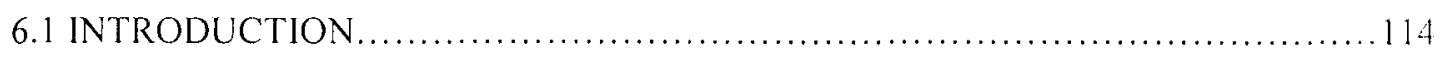

6.2 InGaN GROWTH ON GaN NANOWIRE SUBSTRATES $\ldots \ldots \ldots \ldots \ldots \ldots \ldots \ldots \ldots 114$

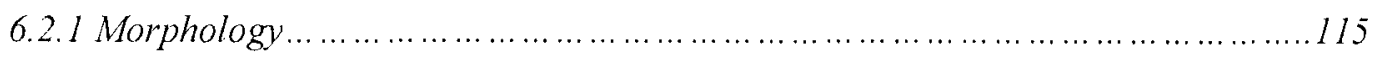

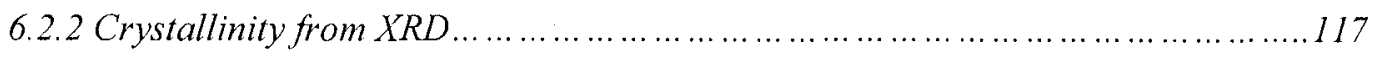

6.2 .3 Band gap from diffuse reflectance ......................................... 119

6.2.4 Epitaxy and composition form electron diffraction ............................ 121

6.2.5 Uniformity of Indium/Gallium distribution in InGaN growth......................126

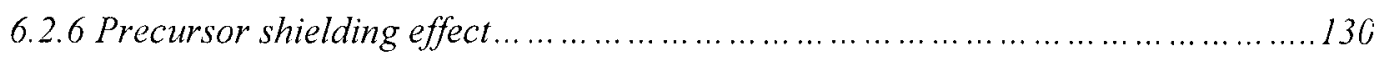

6.2.7 Orientation dependent heteroepitaxial growth of InGaN on GaN NWs ............. 133

6.3 PHOTOACTIVITY OF InGaN LAYERS ...................................... 142 


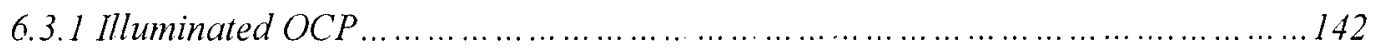

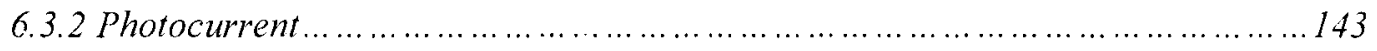

7. GaSbN: A NOVEL MATERIAL SYSTEM............................... 148

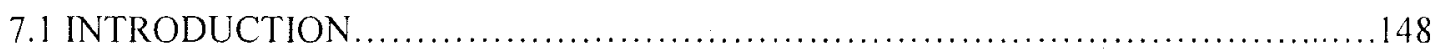

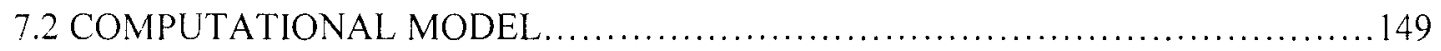

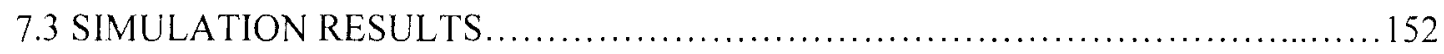

7.4 IMPLICATIONS OF BAND GAP REDUCTION ................................ 155

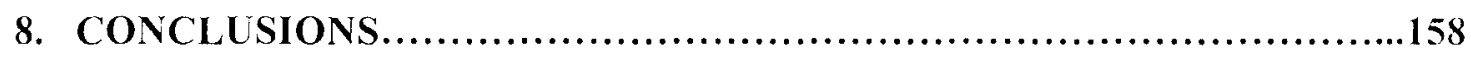

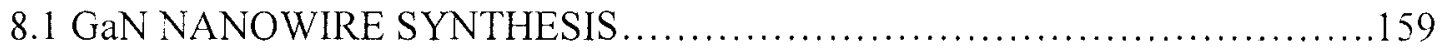

8.2 REACTOR MODELING ................................................ 159

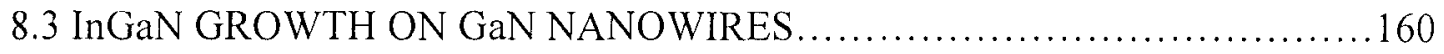

8.4 PHOTOACTIVITY MEASUREMENTS ................................. 160

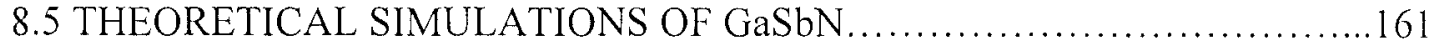

9. RECOMMENDATIONS.................................................... 162

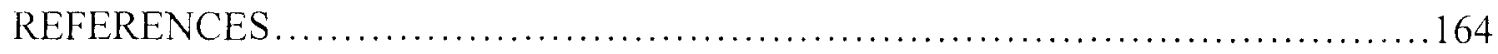

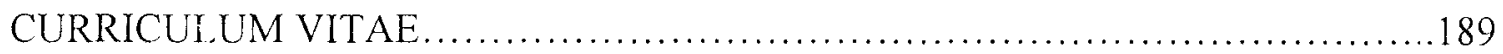




\section{LIST OF FIGURES}

Figure 1.1: Schematic of the band bending and the water splitting process in a semiconductor under illumination. Generation of electron hole pairs is followed by the separation of charges to the two electrodes where the complimentary water redox reactions occur. The barrier causes the majority charge carriers to flow to the counter electrode and the minority carriers drive a reaction at the semiconductor electrode. Oxygen evolution occurs on the a) n-type electrode while hydrogen evolution occurs on a b) p-type semiconductor surface.

Figure 1.2: Schematic of the straddle required for a material to drive the water splitting reaction. For an n-type material, the electrons would drive the hydrogen evolution reaction and holes with act to drive the oxygen evolution reaction.

Figure 1.3: Schematic of the various compensatory architectures for PEC water splitting. a) Two semiconductor p-n cell. b) PV compensated PEC tandem cell and c) Dual PV metal electrode cell.

Figure 1.4: Dual material PEC cell designs a) Schematic of a PEC cell with a p-type and n-type electrode. Minority charges from each electrode drive the two water splitting reactions. Materials must be selected such that the minority band edge straddles one of the redox potentials. 
b) A stacked electrode where the materials have to be selected with proper conductivity type and band alignment such that majority carriers are transported without barriers to the back contact and minority carriers are transported to the surface.

Figure 1.5: Schematic illustrating the advantage of nanowire architecture over planar thin film in terms of increased surface area available for reactions and smaller length scale for minority carriers to reach the surface (reducing recombination).

Figure 1.6 a) Schematic of the ideal nanowire growth pattern on metal substrates. The vertical arrays would help in a uniform growth of the InGaN layers. b) The central GaN nanowire core would be coated with a singie crystalline shell of InGaN of the desired band gap (composition). Finally, a protective coating can be grown on the $\operatorname{InGaN}$ to protect from any corrosion.

Figure 2.1 Schematic of the band alignment of the double sided CdSe and $\mathrm{CdS}$ coating on the $\mathrm{ZnO}$ nanowire arrays. The two materials act as visible light absorbers and inject electrons into the $\mathrm{ZnO}$ scaffold ${ }^{73}$. Similar approach of sensitization has also been studied for $\mathrm{ZnSe}$ on $\mathrm{ZnO}$ nanostructures ${ }^{75}$ which showed much improved performance under visible light illumination. Figure 2.2 Schematic of the reaction processes in a) MBE and b) MOCVD growth. In MBE, the pure metal atoms impinge onto the substrate and react on the substrate at high temperature to form the product. b) In MOCVD, the reactive precursors start reactions and partial pyrolysis form adducts and 
particles in gas phase. The surface reactions are carried out by adducts (adatoms) that are not pure metal atoms which then have different reaction properties and diffusion rates than in MBE.

Figure 2.3 Generic schematic of the MOCVD growth process showing the different growth regimes.

Figure 2.4 Phase diagram of the InGaN alloy system showing the immiscibility gap. The binodal curve shows the instability of the alloy extending throughout the composition range. The dashed spinodal line is indicative of spontaneous segregation. Although narrower in spread, runs through the entire composition. Under the normal synthesis conditions, the alloys with $40-55 \%$ indium composition are thermodynamically unstable ${ }^{43}$.

Figure 2.5 Dark field TEM images of InGaN layers on GaN with a) $12 \%$ indium showing a weak speckled contrast and b) $22 \%$ indium showing bands with higher contrast ${ }^{133}$.

Figure 2.6 Schematic of the mechanism of the formation of "v" defects on InGaN quantum wells a) led by the stacking faults (at high indium content) and b) by the threading dislocations from the substrate into the epi layers ${ }^{137}$.

Figure 2.7 Manifestations of strain relaxation in InGaN films on GaN substrates.

a) Formation of displaced triangular sections due to slip along the planes

b) formation of dislocation half loops via the introduction of misfit dislocations.

Figure 2.8 Manifestations of strain relaxation in InGaN films showing 
the role of dislocations in the substrates. a) Propagation of dislocations from the substrate into the growing film forming pyramidal pits for growth on regular substrate while b) Formation of periodic array of misfit dislocations for growth on ELOG grown films.

Figure 2.9 Observed morphology of InGaN growth on GaN substrates.

a) Small pits formed due to the opening of dislocations at the InGaN/GaN interface. b) Large pits running all the way into the GaN layer, formed due to the opening of the dislocations at the GaN/Sapphire interface. c) pits formed due to thermal erosion.

Figure 3.1 Schematic of the reactor illustrating the heater, substrate and source material position. In the case of GaN powder synthesis, only gallium metal was placed on the susceptor while for c-GaN nanowires, GaN powder along with the substrate and for a-GaN nanowires gallium metal and the substrate were used.

Figure 3.2 Schematic of the reactor illustrating the heater, source and substrate set up for III-Sb nanowire synthesis.

Figure 3.3 Schematic illustrating the instrument and principle of Diffuse Reflectance measurement.

Figure 3.5 a) SAED spot pattern from the GaN nanowire in b) tilted to $[100]$ zone axis.

Figure 3.6 Schematic of the formation of discs in CBED.

Figure 3.7: a) is a sample CBED pattern from the bright spot on a GaN 
nanowire in b).

Figure 3.8 Schematic of the appearance of the electrode after the glass

sheath is attached and all the epoxies are applied and cured.

Figure 3.9 Schematic of the illuminated OCP measurement to check the photoactivity of the sample.

Figure 3.10 Schematic of the three electrode set up used for photocurrent measurements. InGaN/GaN on stainless steel is the working electrode and a platinum wire is the counter electrode with a $\mathrm{Ag} / \mathrm{AgCl}$ reference electrode.

Figure 4.1 Schematic of the MOCVD reactor system. The precursor delivery system is equipped with independent controls for the supply of four precursors. The reaction chamber is a water cooled stainless steel vessel. The gases enter from the top via a showerhead for uniform precursor distribution onto a substrate heated using an induction heater.

Figure 4.2 Schematic of the precursor delivery system.

Figure 4.3 is the schematic of the bubbler and the gas pressure and flow control setup used to control the precursor flux.

Figure 4.4 a)-b) Schematics of the top plate of the reaction chamber.

Two isolated concentric groves are made in the plate. The plate has two isolated chambers for water cooling (connections at the top) and showerhead via a perforated stainless steel plate (opens at the bottom). c) and d) are the schematics of the bottom plate showing a similar water cooling chamber as well as the teflon sleeve for a vacuum tight sealing of the copper tube of the induction heater. The separation between the showerhead (fixed) and 
the substrate is changed by moving the heater assembly.

Figure 4.5 Schematic of the reaction chamber with the heater assembly

showing the chamber, the showerhead, the substrate placement, the Teflon

sleeve holding the copper tubing containing the copper coils for induction

heating using the circuit box at the bottom.

72

Figure 4.6 Schematic of the vertical stagnation flow system showing the

flow pattern of the precursors from the showerhead to the substrate.

Figure 4.7 Meshing of the symmetric half of the reactor. The regions of interest (close to the substrate and walls) are meshed tightly and the volume is under a relaxed mesh.

Figure 4.8 Representative 3 -d plot showing the dependence of growth rate as a function of the parameters described in table 4.2. All the plots have temperature in the $\mathrm{x}$ and distance from the center of the substrate on the $\mathrm{y}$ axis.

Figure 4.9: Comparison of growth rates of $\mathrm{GaN}$ as a function of material flux.

The flow rate of carrier gas $\mathrm{N}_{2}$ was increased three times from $5 \mathrm{sccm}$ to 15

sccm. A linear change in growth rate is observed.

Figure 4.10 Comparison of growth rates as a function of temperature.

The growth rate shows a very little increase with temperature (almost constant at small separation distances) with the effect being pronounced at higher pressures and at larger distances from the center of the substrate. Figure 4.11: Effect of substrate showerhead separation on the growth rate and uniformity of deposition. The growth rate drastically drops from $0.5 \mathrm{inch}$ to 1.5 inch at the center but is almost constant at the ends of the substrate. 
Figure 4.12 SEM images of the InGaN growth on GaN nanowires showing the effect of substrate and showerhead separation on the growth rate. The growth at a) 4 inch separation is only $100-125 \mathrm{~nm}$ in 15 minutes while the growth at b) 1 inch separation is about 250-350 nm thick for the same duration. The scale bar in the images is $500 \mathrm{~nm}$.

Figure 4.13 Growth rate plots at different separation, pressure and temperature for $5 \mathrm{sccm}$ N2 flow.

Figure 4.14 Growth rate plots at different separation, pressure and temperature for $15 \mathrm{sccm} \mathrm{N}_{2}$ flow.

Figure 5.1 Schematic illustrating the process sequence of catalyst assisted VLS growth. The catalyst droplet causes the cracking of the gas phase species and dissolution of the growth species and further dissolution causes supersaturation. Precipitation occurs at the liquid substrate interface leading to the tip led growth of a nanowire.

Figure 5.2: SEM micrographs of the two different nanowire growth modes. a) Tip led growth of silicon nanowires. b) Bulk nucleation of bismuth nanowires out of one gallium droplet.

Figure 5.3 Binary phase diagrams of a) Ga-Si and b) Au-Si depicting the very small and large eutectic compositions in the two systems.

Figure 5.4 Phase diagram of the Ga-Sb system. The three regions are marked for being very different in eutectic composition and equilibrium solubility to study the role of the same in controlling nanowire growth mode. Region 1 and region 3 are the two eutectics at excess gallium and 
excess antimony regions. Region 2 is markec as the range for gallium tip led growth.

Figure 5.5 a) SEM micrograph of the GaSb nanowires synthesized on quartz substrates under antimony rich conditions. b) Magnified view of the GaSb nanowires showing the tip. c) High resolution TEM image of the nanowire showing the single crystalline nature of the nanowire. d) FFT pattern of the nanowire showing the $<101>$ growth direction. e) Raman spectrum of the GaSb nanowires showing the characteristic $229 \mathrm{~cm}^{-1}$ peak. f) NIR PL emission from the GaSb nanowires showing peak at $0.73 \mathrm{eV}$ the characteristic band edge emission of $\mathrm{GaSb}^{175}$. Figure 5.6 TEM EDS line scan along the GaSb nanowire and the tip. The nanowire is $\mathrm{GaSb}$ with equal and uniform distribution along the length while the tip is almost pure antimony and very little residual gallium in the same proportion as the eutectic composition.

Figure 5.7 Estimated critical nuclei diameters for GaSb system for various antimony compositions. Regions 2 and 3 with the large equilibrium solubility have large critical nuclei sizes and region 1 with low solubility has much smaller critical nuclei size.

Figure 5.8 SEM EDS elemental map of GaSb nanowire. The absence of antimony at the tip and it showing up in the gallium map is a clear indication of the tip being pure gallium. The scale bar is $1 \mu \mathrm{m}$. 100 Figure 5.9 Phase diagram of the In-Sb system showing the two eutectics $(\sim 1 \%$ solubility in indium rich conditions and $32 \%$ solubility 
in the antimony rich region).

Figure $5.10 \mathrm{SEM}$ micrographs of the as synthesized InSb nanowires where the tip led growth is clearly evident. The scale bar is $100 \mathrm{~nm}$.

Figure 5.11 SEM EDS elemental map of InSb nanowire showing excess antimony at the tip indicating an antimony tip led growth of the nanowires.

The scale bars are $100 \mathrm{~nm}$.

Figure 5.12 Template for process and substrate selection for controlled growth of GaN nanowires. Metal substrates can always be used to grow nanowires using the developed process window whereas nanowire growth on semiconductor or insulators is heavily affected by the wetting behavior of gallium.

Figure 5.13 SEM micrograph of a) "c" plane and b) "a" plane oriented GaN nanowires synthesized on stainless steel.

Figure 5.14 SEM micrographs of "a" plane oriented GaN nanowires synthesized on quartz substrates.

Figure 5.15 SEM micrographs of "c" plane oriented GaN nanowires grown on copper substrate.

Figure 5.16 SEM micrographs of "c" plane oriented GaN nanowires synthesized on GaN substrate 108

Figure 5.17 SEM micrographs of "c" plane oriented GaN nanowires synthesized on silicon substrate.

Figure 5.18 SEM images of the coiled GaN nanowire morphology of GaN grown using copper tips. The nanowires are 10 s of microns in length 
ard $10-50 \mathrm{~mm}$ in diameter. The coil could be the result of constant copper droplet instability on the growing nanowire.

Figure 5.19 SEM images of the copper catalyzed GaN nanowires showing the straight nanowire growth suitable for vertical array format. The nanowires are $\sim 100 \mathrm{~nm}$ in diameter and $2-5 \square \mathrm{m}$ in length. Faceting of the tip is clearly evident.

Figure 5.20 SEM images of the branched growth on straight GaN nanowires. The branches are also copper tip led with diameters in the 10-20 $\mathrm{nm}$ range. The branches are straight to some length and then bend $120^{\circ}$ possibly due to droplet instability.

Figure 5.21 HRTEM images of the $120 \mathrm{o}$ bent GaN nanowire branch. The arm as well as the bend does not have any stacking faults/kinks. The orientation of the nanowire is preserved as GaN has a six fold symmetry which also could be the reason for the observed bend angle of the nanowire.

Figure 6.1 SEM micrograph of the evolution of InGaN growth a)-c) c-GaN and d)-f) a-GaN nanowires. Island growth, coalescence followed by a shell formation is the general observation. The hexagonal islands grow along the length of the $\mathrm{c}-\mathrm{GaN}$ nanowires while growth is perpendicular to the length in the case of a-GaN nanowires. Figure 6.2 Compilation of the XRD peaks obtained from the synthesized InGaN alloys. XRD peaks from $\mathrm{GaN}$ and $\mathrm{InN}$ are included to show that the peaks lie within the two composition extremities. 
Figure 6.3 Compilation of all the band gaps measured on the InGaN

samples measured via diffuse reflectance the band gaps of all

the $\mathrm{InGaN}$ alloy compositions from $0.7 \mathrm{eV}$ to $3.4 \mathrm{eV}$.

Figure 6.4 Representative HRTEM image of the InGaN growth on GaN

nanowire. InGaN layer has a larger lattice constant that the GaN nanowire

as indicated in the figure.

Figure 6.5 Sample SAED spot patterns obtained from InGaN layers on

GaN nanowires. The difference in the patterns is the relative separation

of the spots which is indicative of heteroepitaxial structures with different

lattice parameters. Presence of only two identical symmetric sets of spot patterns

shows single crystalline growth.

123

Figure 6.6 Representative SAED pattern with an intensity profile showing the two sets of peaks. Peaks closer to the center are InGaN and GaN peaks are farther from the center. Spots chosen are within the red rectangle in the SAED pattern.

Figure 6.7 Compilation of the intensity profiles from the SAED patterns.

All the measurements are normalized wrt GaN peak. The increased spacing

of the InGaN peaks from the GaN peak is a measure of the increasing

shift in lattice constant translating to increasing indium content InGaN alloys.

We have synthesized InGaN alloys from InN all the way to GaN. Each

InGaN peak is labeled with two numbers. The first is the estimated

Indium content measured from the peak positions and the

second number is the composition measured from TEM EDS.

125 
Figure 6.8 TEM EDS elemental map of InGaN growtin on "c" plane oriented GaN nanowire. The nanowire is clearly visible in the gallium map and leaves a conspicuously blank pattern in the indium map. The uniform distribution of gallium and indium is clear in the maps. An image of the InGaN islands on GaN nanowire is also included for comparison.

Figure 6.9 TEM EDS elemental map of InGaN growth on "a" plane oriented $\mathrm{GaN}$ nanowire. The nanowire is clearly visible in the gallium map and leaves a conspicuously blank pattern in the indium map. The uniform distribution of gallium and indium is clear in the maps. An image of the InGaN islands on $\mathrm{GaN}$ nanowire is also included for comparison.

Figure 6.10 Measured lattice parameters of $\mathrm{InGaN}, \mathrm{GaN}$ and $\mathrm{InN}$ via CBED. The measured compositions show that on nanowires from one sample there is a minor composition variation and overall it matched with that measured from other techniques. All the measurements are consistent with the characteristic wurtzite c/a ratio 1.6 .

Figure 6.11 SEM micrograph of the extremely high density of GaN nanowires. The density gradient increasing from the top to the bottom (substrate surface) is also stown.

Figure 6.12 SEM micrographs of the variation in InGaN growth as a function of the distance from the top demonstrating the shielding of the precursor flux reaching the lower layers due to the high density of the nanowires. The wires on the top layer have a uniform conformal coating but the growth tends to be more scattered and island like in the middle 
regions. At the lower region, close to the substrate, there is practically no growth of InGaN.

Figure 6.13 Schematic of the hexagonal GaN crystal structure. c-GaN nanowires have a hexagonal cross section bounded by six non polar (10-10) facets while a-GaN nanowires have a rectangular cross section bounded by alternating pairs of polar (0001) and non-polar (10-10) facets.

Figure 6.14 Schematic of the growth mechanism of InGaN growth on GaN nanowires. a) InGaN growth on a-GaN nanowires leads to a nonpolar hexagonal shell on the nanowire while b) growth on a-GaN nanowire leads to the formation of a semi-polar shell on the nanowire.

Figure 6.15 SEM micrograph of the InGaN growth on GaN nanowires. Islands with different sizes are shown indicating that the islands form at the very initial stages followed by the island growth at constant aspect ratio.

Figure 6.16 Schematic of the two possible views of the hexagonal pyramidal islands. The base is equal to 1.72 times the lattice constant " $a$ " when only two facets are visible whereas the measured base would be twice the lattice constant "a" when three facets are visible.

Figure 6.17 Aspect of the pyramidal islands of different sizes and different InGaN compositions. Number of facets of the pyramid visible in the plan view controls the ratio. The steps form a pyramidal shape at very initial stages and grow in size maintain the aspect ratio. The size of the pyramids has been measured from $<5 \mathrm{~nm}$ (smallest) to $>100 \mathrm{~nm}$ (largest), corroborating our proposed growth mechanism. 
Figure 6.18 Iiluminated open circuit potential (photo-voltage) measurements on InGaN layers on GaN nanowires. The potentials become more negative upon illumination which indicates the materials being n-type. The measured OCP is constant over time reflecting on the stability of the material.

Figure 6.19 Photocurrent measurements on the In $\mathrm{GaN} / \mathrm{GaN}$ electrode. Potential scan was performed from $1 \mathrm{~V}$ to $-0.5 \mathrm{~V}$ vs OCP at a scan rate of $10 \mathrm{mV} / \mathrm{sec}$. Photocurrent onset occurs at about $0.45 \mathrm{~V}$ wrt $\mathrm{Ag} / \mathrm{AgCl}$. All the measurements were conducted at an illumination intensity of $100 \mathrm{~mW} / \mathrm{cm}^{2}$

Figure 6.20 Photocurrent obtained in two samples prepared under the same conditions. The reduced current in the second sample indicates a high resistance contact.

Figure 6.21 Photocurrent as a function of illumination intensity. A linear increase is observed with illumination intensity indicating that the carrier generation is the limiting step.

Figure 6.22 Test of stability of the electrode over time. The photocurrent is almost constant over a period of $5 \mathrm{hrs}$ which indicates no degradation of the material.

Figure 6.23 SEM images of the nanowires (a) before and (b) after PEC testing. The InGaN layer needs to be tested for composition to determine the extent of photocorrosion if any.

Figure 7.1 a) Optimized 72-atom wurtzite GaN supercell with two substitutional $\mathrm{Sb}$ atoms (in green). b) Optimized 48-atom zinc blende GaSb supercell 
with two substitutional $\mathrm{N}$ atoms (in blue) ${ }^{195}$.

Figure 7.2 Calculated band gaps for GaSbxN1-x and $\mathrm{GaSb}_{1-\mathrm{x}} \mathrm{N}_{\mathrm{x}}$ alloys as a function of concentration ${ }^{196}$. Experimentally reported band gap values for $\mathrm{GaSb}_{1-x} \mathrm{~N}_{\mathrm{x}}$ alloys are also included for comparison.

Figure 7.3 a) Calculated band structure, allowed optical transitions and b)

PDOS for the GaSb $\mathrm{N}_{1-x}(0.05<x<0.06)$ alloy $^{196}$.

Figure 7.4 A qualitative comparison of the band edge of GaSbN alloys wrt the hydrogen and oxygen water splitting potentials. The $\mathrm{GaSb}_{1-x} \mathrm{~N}_{\mathrm{x}}$ band edge positions are plotted using values from literature whereas the $\mathrm{GaSb}_{\mathrm{x}} \mathrm{N}_{1-\mathrm{x}}$ values are the calculated values in this study ${ }^{196}$. It is evident that the $\mathrm{GaSb}_{\mathrm{x}} \mathrm{N}_{1-\mathrm{x}}$ alloy straddles the water splitting reaction potentials. 
CHAPTER 1

\section{INTRODUCTION}

\subsection{Energy and Environmental Challenges}

The demand for energy has been on the rise since the beginning of industrial age. According to the US Department of Energy, the energy demand has risen from 0.7 TW in $1900^{1}$ to $14 \mathrm{TW}$ in $2005^{2}$ and according to the current trends, the demand is projected to double by $2050^{3}$. The heart of the problem is that the primary source of our energy supply is fossil fuels: coal, natural gas and oil. It has been estimated that $87 \%$ of the total supply of energy in 2005 was from fossil fuels ${ }^{3}$. Coal and natural gas are primarily used for heating and electricity generation while the liquid hydrocarbon fuels are used for transportation applications. Current known resources of coal and natural gas are

estimated to last for atleast 118 years $^{4}$ at the current rate of usage. Estimations of how long the hydrocarbon fuels will last is flawed as there is much speculation over the actual reserves and the currently discovered reserved but the currently available reserves are expected to last for another 50 years. This however is a debatable figure with reports of discoveries of new non-conventional reserves. 
Problem with these numbers is the geographic and dynamically changing demographics that are leading to a very unstable geopolitical situation. The major producers are not the major consumers ${ }^{5,6}$ which along with very unstable governments in countries with major oil reserves make the whole situation very volatile. This situation is strained further by increasing world population ${ }^{7}$ wherein the majority of the increase is concentrated in India and China ${ }^{8}$ which creates a supply and demand imbalance. It is also a concern for USA in terms of energy security as it has only about $3 \%^{6}$ of total oil reserves and is the highest energy consumer in the world ${ }^{9}$ with increasing energy use. The numbers would be a little different in the rest of the world but specific to USA, petroleum accounted for $47 \%$ of the total fossil fuel based energy consumption with coal and natural gas accounting for $27 \%$ and $26 \%$ respectively ${ }^{10}$.

Burning of the fossil fuels releases copious amounts of $\mathrm{CO}_{2}$ and $\mathrm{CO}$ which are greenhouse gas that causes global warming. Hence, an increased dependence on fossil fuels translates to increased greenhouse gases emission. Industrialized countries as well as rapidly growing economies ${ }^{11}$ are putting immense strain on the natural balance by pumping huge amounts of $\mathrm{CO}_{2}{ }^{12,13}$. Hence, the dwindling fossil fuels along with the associated environmental problems make it imperative to look for alternative sources of energy.

The available alternative sources ${ }^{14}$ of energy are solar, wind, tide, geothermal and nuclear. Of these, nuclear energy is promising but concerns related to safety and spent fuel handling limits its mass acceptance. Geothermal energy is natural but is very location specific and only a very small fraction can be extracted profitably ${ }^{15}$. Similar cases can be made out of hydro, tidal and wind energy where they can be useful only in specific 
locations and are also intermittent. Such systems can be an aid to the grid but cannot be solely depended upon.

Out of all the sources, solar energy is the most widely available and inexhaustible source and is in so much abundance that it can provide for all the energy needs of the world. The vastness of solar energy hitting earth can be estimated by the fact that the energy from sun in a year will be twice as much as the "TOTAL" energy from all available reserves of non-renewable sources of coal, oil, gas and uranium combined ${ }^{16}$. In other words, solar energy reaching the earth surface is $89000 \mathrm{TW}^{17}$ compared to the current usage of $14 \mathrm{TW}$ and will be able to handle any projected increase in demand. There are a variety of ways of utilizing solar energy. The most widely used is solar cells where the sunlight is directly absorbed by a semiconductor material to create electron hole pairs which are then separated to generate electricity. This is a direct way to use solar energy and has met with considerable success. However, the intermittent nature of sunlight makes it imperative to have a solar cell in conjunction with an efficient energy storage auxiliary to store energy during daytime for use when light is not available. Also, a direct current (DC) is generated in a solar cell which has to be converted to alternating current $(\mathrm{AC})$ which leads to $4-12 \% \operatorname{losses}^{18}$. The most important limitation for portable applications of solar cells is the where the limited cell area and the high energy density required for transportation. Solar cells can be used to charge batteries for transportation but changing the entire infrastructure for battery driven transport is not feasible as the battery technology is still in development phase with very limited range of operation and are slow to charge. Also, solar thermal can has been studied to be a viable option but it requires expensive high temperature resistant materials, is geographically limited and 
cannot be used for transportation ${ }^{19}$. A prudent route would be to use an alternate high energy density fuel with minimal carbon footprint.

Solar energy can also be used to create a reactive system where high energy density fuels can be synthesized as energy carriers. The created fuels can be used on demand for various applications. Solar energy can be used to create hydrogen from $\mathrm{H}_{2} \mathrm{O}$ or methanol from $\mathrm{CO}_{2}$. Of the two available options, a hydrogen based economy has been shown to be viable ${ }^{20}$ as $\mathrm{H}_{2}$ is clean and if used in a fuel cell is completely free of carbon. The methanol cycle involves carbon which is not the complete solution to the problem. Hence, using hydrogen looks to be one of the most promising options.

\subsection{Issues with hydrogen production}

In the current HYDROCARBON economy, petroleum is used as the primary fuel for all energy needs. Combustion is the main reaction where copious amounts of carbon dioxide and carbon monoxide are always produced apart from many other undesirable gases.

Hydrogen has long been regarded as the solution to the problems associated with our excessive dependence on fossil fuels. The finite availability of the primary fuel coal was recognized as early as 1874 by Jules Verne where he made the comment that "water will be the coal of the future"21 hinting on the use of hydrogen derived from water electrolysis. The use of hydrogen (derived from water) as a transportation fuel was

suggested in the 1930s by Rudolf Erren ${ }^{22}$ with the goal to reduce automotive emissions and reduce oil imports into England. As early as 1973, Francis Bacon suggested the use 
of hydrogen as an energy storage system ${ }^{23}$. Although hydrogen was being looked as an alternative to fossil fuels very early in the game, lack of required technology, the huge availability of oil along with the ever growing need for fuel to maintain the rapid industrial growth meant that fossil fuels took were preferred. It was only the realization that fossil fuels lead to excessive pollution and greenhouse gas emissions that sent scientists scrambling for clean and renewable energy alternatives. John Turner has made some very thorough and insightful points in his commentary on sustainable hydrogen production where he points out the systemic problems with some hydrogen production methods and suggests some options to move forward towards a hydrogen economy ${ }^{20}$.

Hydrogen is clean, abundant and when used in fuel cells to generate electricity is carbon free. However, the current production methods are the reason people are skeptical about large scale implementation of hydrogen based technologies. Current hydrogen production methods include steam reforming and water-gas shift reaction as shown in equations 1.1 and 1.2

$$
\begin{aligned}
& \mathrm{CH}_{4}+\mathrm{H}_{2} \mathrm{O} \rightarrow \mathrm{CO}+3 \mathrm{H}_{2} \\
& \mathrm{CO}+\mathrm{H}_{2} \mathrm{O} \rightarrow \mathrm{CO}_{2}+\mathrm{H}_{2}
\end{aligned}
$$

As seen in the reactions, carbon dioxide and carbon monoxide are also produced that add to the carbon footprint. This defeats the purpose of using hydrogen with the motivation of using a clean fuel. The idea of an onboard reforming system coupled with a fuel cell for transportation application was rejected since the cost of producing hydrogen by reforming is low at industrial scale but becomes substantial at the small scale for portable applications ${ }^{24}$. Hence, cost and non-zero carbon emissions make this approach 
impractical. Hydrogen is also produced from sources other than fossil fuels. Biomass reforming and water electrolysis are the most popular in this regard. Biomass reforming again is a carbon based process which apart from the carbon based emissions also creates novel issues. The cultivation of biomass will become more expensive with the increase in commercialization. Also, this approach also has the same issues with large scale storage and transportation for portable/transportation solutions. Water electrolysis is a direct method where water molecules are split into the constituent gases by passing electric current. Electricity from any source can be used to drive the process. Since water electrolysis in an energy intensive and inefficient process, utilizing energy from grid makes the entire process 3 to 10 times more expensive than hydrogen produced from coal and natural gas ${ }^{25}$.

Solar Cells can be used to provide the electricity needed to drive the reaction but the process with one photovoltaic and an electrolytic component becomes a balance of system problem with reduced efficiency whereas a direct system with photocatalyst to directly split water would be more efficient ${ }^{26,27}$. Water can also be split directly at high temperatures (1000-1200 C) by a SOLARTHERMAL process. The system requires materials that can withstand temperatures close to $2000 \mathrm{C}$ making it very expensive due to the exotic materials needed. Recently, the development ${ }^{28}$ of a novel membrane reactor technology has reduced the temperature constraints. $100 \mathrm{~m}^{2}$ of solar thermal system can produce 1 kilogram of hydrogen per hour. However, the technology is still in development and needs complex solar tracking and high precision and quality reflectors to attain the temperatures. Point being: it is still a very small scale and localized 
production system and a very costly one at that, still retaining all the storage and transportation issues.

All the methods of hydrogen production discussed so far are either expensive, inefficient or carbon intensive. The main arguments against the hydrogen economy are the means of hydrogen generation and storage technologies. The current methods are still hydrocarbon dependent or are too expensive. Also, they are heavily reliant on extensive balance of systems. New carbon independent methods need to be developed to enable the hydrogen based systems viable. One of the most promising solutions that merit discussion is photoelectrochemical (PEC) water splitting.

\subsection{Photoelectrochemical water splitting}

\subsubsection{PEC water splitting: Basics}

PEC water splitting is a process where a semiconductor material generates charge carriers upon illumination which then drive the water splitting process. Before going into the details of the process, it is important to understand the basic phenomena of semiconductor electrolyte interface. The system consists of a semiconductor electrode and a metal counter electrode immersed in an electrolyte solution. The bands (conduction and valence) of a semiconductor are flat when not in contact with another material (liquid or solid). In this state, the position of the Fermi level, closer to the conduction or the valence band, is determined by the material being $\mathrm{n}$ or $\mathrm{p}$ type.

Once the semiconductor is immersed in the electrolyte, the Fermi levels equilibrate i.e. charge balancing occurs. An n-type semiconductor, where the Fermi level 
is closer to the conduction band, loses electrons into the electrolyte. This effectively moves the Fermi level down. Since the position of the bands is pinned at the semiconductor-electrolyte interface, as shown in the schematic 1.1, band bending occurs. The amount of bending is a function of the dopant density in the material as well as separation of the two Fermi levels. For an n-type semiconductor, since the Fermi level moves down upon equilibration, the bands bending as shown in figure 1.3aa is termed as upward. As the electrons migrate into the electrolyte, the charges balance and there is a field now to prevent any more electron transfer into the electrolyte. A similar process occurs for p-type semiconductors where the band bending is downward as the Fermi level has to rise at equilibration as shown in figure $1.1 \mathrm{~b}$.

In a semiconductor, electrons are in the valence band and the conduction band states are unfilled. Upon excitation by energy equal to or greater than the band gap, the electrons from the valence band cross over to the conduction band to an excited state. As the electrons leave the valence band, to counter the induced charge imbalance, holes are generated in the valence band. There are as many holes in the valence band as there are excited electrons in the conduction band. Now, the electrons have to relax since they are excited. The relaxation process requires transfer to a lower energy state. The electrons can recombine with the holes in the valence band by releasing energy equal to the band gap which is known as luminescence. 
(a)

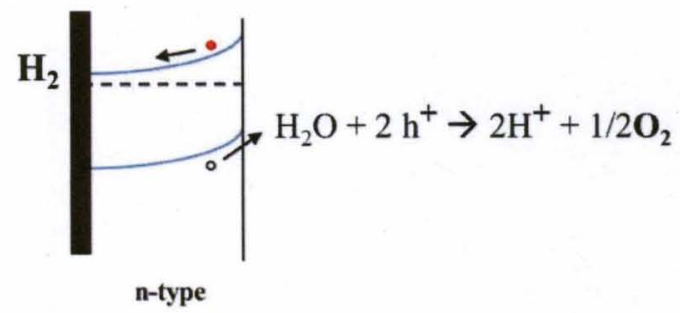

(b)

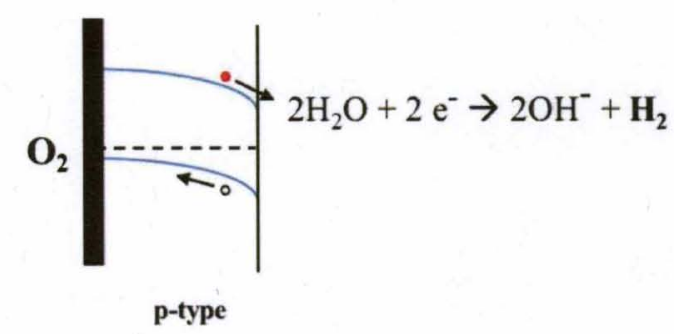

Figure 1.1 Schematic of the band bending and the water splitting process in a semiconductor under illumination. Generation of electron hole pairs is followed by the separation of charges to the two electrodes where the complimentary water redox reactions occur. The barrier causes the majority charge carriers to flow to the counter electrode and the minority carriers drive a reaction at the semiconductor electrode. Oxygen evolution occurs on the a) n-type electrode while hydrogen evolution occurs on a b) p-type semiconductor surface.

The water splitting process involves using the excited electrons in the conduction band and the holes in the valence band to drive the water splitting reactions. If the transport through the material is fast enough wherein the electrons/holes move to the other electrode and drive the water splitting reaction, the loss due to recombination is prevented. Equation 1.1 and 1.2 show the water splitting reactions 


$$
\begin{aligned}
& \mathrm{H}_{2} \mathrm{O}+2 \mathrm{~h}^{+} \rightarrow 2 \mathrm{H}^{+}+1 / 2 \mathrm{O}_{2} \\
& 2 \mathrm{H}_{2} \mathrm{O}+2 \mathrm{e}^{-} \rightarrow 2 \mathrm{OH}^{-}+\mathrm{H}_{2}
\end{aligned}
$$

The two redox reactions have to be driven simultaneously and the gases evolve at the two electrodes depending on the conductivity type of the material as shown in figure 1.2. A careful look at the process makes is very clear that the semiconductor is the most important part of the entire system. A semiconductor must satisfy a number of band gap, band edge and transport criteria to be considered a potential PEC water splitting material. An insightful review on the PEC water splitting process and the state of the art has been presented by $\mathrm{T}$ Bak and co-authors in their review article on water splitting ${ }^{29}$.

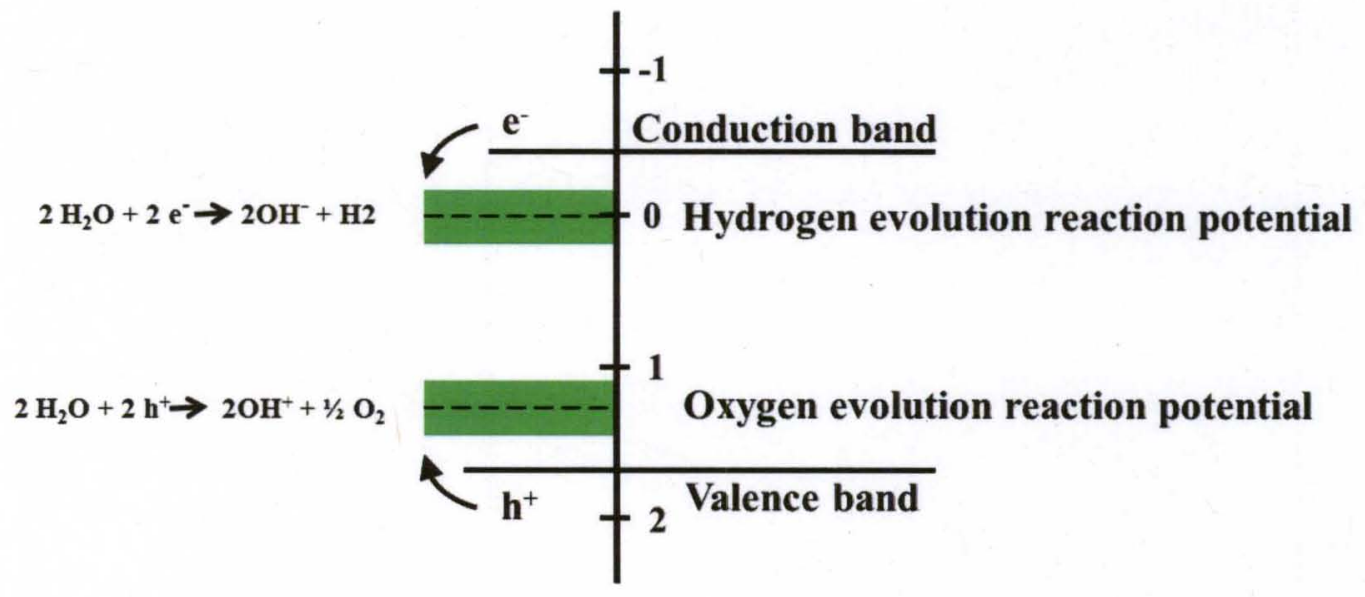

Figure 1.2 Schematic of the straddle required for a material to drive the water splitting reaction. For an n-type material, the electrons would drive the hydrogen evolution reaction and holes with act to drive the oxygen evolution reaction. 


\subsubsection{PEC water splitting: material criteria}

Since the demonstration of photoelectrochemical water splitting by Fujishima and Honda ${ }^{30}$ using $\mathrm{TiO}_{2}$ electrode, researchers have been focused on new materials and devising novel integration techniques for generating $\mathrm{H}_{2}$ from water. The goal has always been to achieve spontaneous (unassisted) water splitting directly from sunlight. Thermodynamically, the conversion of one molecule of $\mathrm{H}_{2} \mathrm{O}$ into $\mathrm{H}_{2}$ and $1 / 2 \mathrm{O}_{2}$ under standard conditions has a free energy change $(\Delta \mathrm{G})$ of $237.2 \mathrm{KJ} / \mathrm{mol}$. According to Nernst equation, this converts to a $\Delta \mathrm{E}^{\circ}=1.23 \mathrm{~V}$. Upon illumination, the generated electron hole pairs should have energy at least equal to $1.23 \mathrm{eV}$ so that they can drive the reactions. However, the losses at the electrode interfaces require about $1.7 \mathrm{eV}$ energy difference between the generated electrons and holes in a material to drive the water splitting reaction. Hence, a semiconductor should have a band gap greater than $1.7 \mathrm{eV}$ to generate charge carriers with sufficient energy.

However, from a practical standpoint, the energy spread of sunlight plays the controlling role in the selection of band gap. Maximum sunlight is in the energy range of 1.7 to $2.2 \mathrm{eV}^{31}$. Hence, it is prudent to use the maximum energy input available to maximize the water splitting efficiency. A direct band gap in the 1.7-2.2 eV range would be a good choice as the maximum solar energy would be available for absorption. Direct band gap allows for the maximum absorption as indirect band gap requires absorption of a phonon as well which makes the process inefficient.

Apart from the band gap, the band edges of the material must have the right energy positions wherein the charge carriers can drive the water splitting reactions ${ }^{32}$. The 
band edges of the material should straddle the water splitting reaction potentials. As shown in the schematic in figure 1.2, the conduction band of the material should be higher than the hydrogen evolution reaction potential and the valence band should be lower than the oxygen evolution potential. The straddle gap is the extra energy for the charge carriers to drive the reactions.

Overall, a material should have a direct band gap in the $1.7-2.3 \mathrm{eV}$ range with the band edges straddling the water splitting reaction potentials. However, these criteria deal with the carrier generation aspect. There are other factors such that actually determine if the material would really work to split water. In a water splitting cell, the semiconductor is the working electrode and a platinum wire is the counter electrode. Upon illumination, the semiconductor absorbs the energy and generates electron hole pairs. The charge carriers would recombine releasing energy if they are not separated. The semiconductor should have excellent charge transport properties wherein the generated majority charge carriers can be transported from the generation sites to the counter electrode via the back contact.

So far, the criteria involved material properties that would allow a material to split water efficiently. The final criteria address the practicality of the material for long term device application. For large scale acceptance of the material for water splitting, the material should be stable in the solution. Most materials with the band gap $<2.3 \mathrm{eV}$ are unstable in solutions and the materials that are stable have a wide band gap that limits the amount of solar energy absorbed. 


\subsubsection{PEC water splitting: Challenges}

Finding the right material is the main problem in the challenge for spontaneous water splitting. So far, no material, neither the binary systems ${ }^{33},{ }^{34}$ (limited by their discrete properties) nor the ternary systems ${ }^{33}, 35$ (band gap can be tailored with composition between the band gaps of the two binary extremities) has been found that can satisfy all the criteria. The materials have either too small or too large band gaps o and if the band gaps are in the right range, the band edges do not straddle wherein extra energy is required to drive the water splitting process. Hence, it is imperative to look for new materials as well as develop new device structures that can make use of the existing materials.

Apart from the direct PEC water splitting using a single material that fits all criteria, compensatory designs can actually be developed so that materials that are partially suitable can be used. In their pioneering work on PEC water splitting using $\mathrm{TiO}_{2}$ electrodes $^{30}$, Fujishima and Honda, mention that using a " $\mathrm{p}$ " type semiconductor as the other electrode instead of platinum may increase the water splitting efficiency. This also points to the need to alter the PEC water splitting cell configuration to achieve higher performance. This is a direct means to remove the expensive metal electrode as well as provide additional carriers at the other electrode to drive the reaction with better efficiency.

The problem with most materials is that the band edges do not straddle. This can be compensated ${ }^{36,37}$ externally by connecting a potential source using a solar cell to supply the additional energy required. Tandem PV-PEC cell architecture ${ }^{38}$ can be 
incorporated to internally compensate the required energy which would need choosing materials with the right band edge positions. The various compensatory architectures are illustrated in figure 1.3. A single material cell would be the preferred alternative as the $\operatorname{architectures}^{37}$ shown in figure 1.3, although work, need a careful selection of materials and the competing reactions have also to be suppressed.

(a)

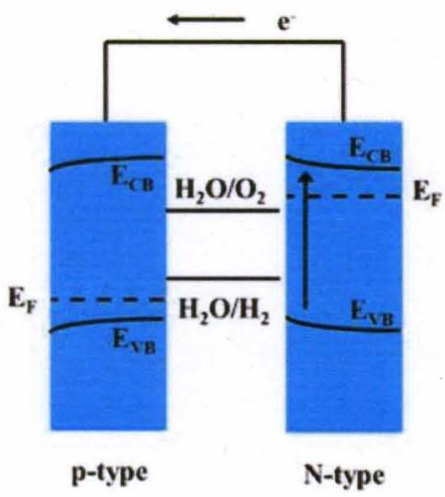

(b)

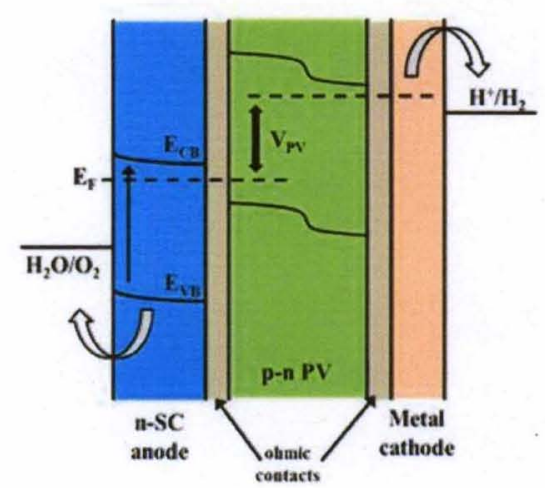

(c)

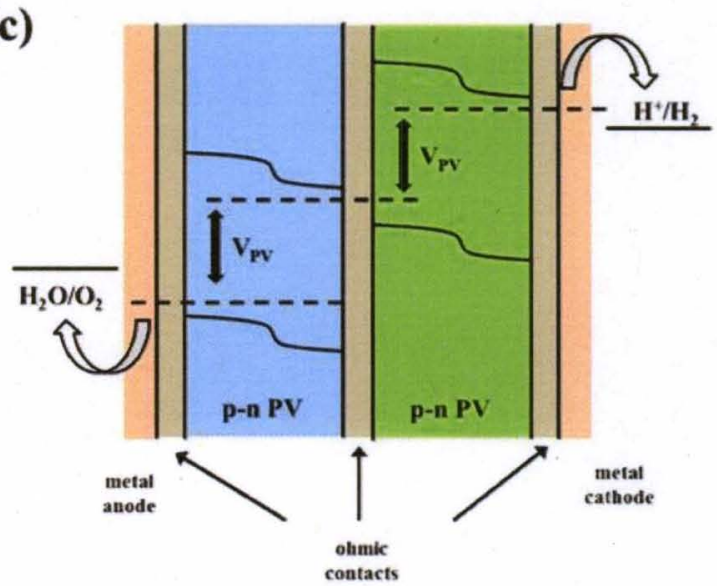

Figure 1.3: Schematic of the various compensatory architectures for PEC water splitting.

a) Two semiconductor p-n cell. b) PV compensated PEC tandem cell and c) Dual PV metal electrode cell. 


\subsection{Materials of Interest}

Our interest spans the search for a single material direct PEC water splitting system (figure 1.2) as well as a compensatory system wherein two different materials or $p$ and $\mathrm{n}$ type of the same material can be used to spontaneously drive the water splitting process (figure 1.3 a). GaN is a wide band gap material that straddles the water splitting potential window ${ }^{39}$ and has been shown to have excellent stability in aqueous solutions. A GaN based ternary alloy is hence expected to have similar properties of its starting material. This is the basis for our interest in InGaN as a potential PEC material. InGaN has a tunable band gap from $0.8 \mathrm{eV}$ to $3.4 \mathrm{eV}$. Although projections of band edges of InGaN show it does not straddle the water splitting potentials, it can be used in a compensatory architecture for water splitting as it has excellent absorbing characteristics.

InGaN can be used in two material system as well as tandem architectures. Two electrode systems with $\mathrm{p}$ and $\mathrm{n}$ type materials can be devised wherein minority band edge of each material straddles one of the water splitting reaction potentials. A combination of $\mathrm{n}-\mathrm{TiO} 2$ and $\mathrm{p}-\mathrm{GaP}$ has been shown to split water without the need for any external bias ${ }^{40}$. However, the efficiencies will be limited due to the high band gap of $\mathrm{TiO}_{2}$. As shown in the schematic in figure 1.4, InGaN alloys with $2 \mathrm{eV}$ band gap can be used as the oxygen evolution electrode as the conduction band is positive to the hydrogen evolution potential. A complimentary material needs to be selected which has the valence band negative to the oxygen evolution reaction. 
(a)

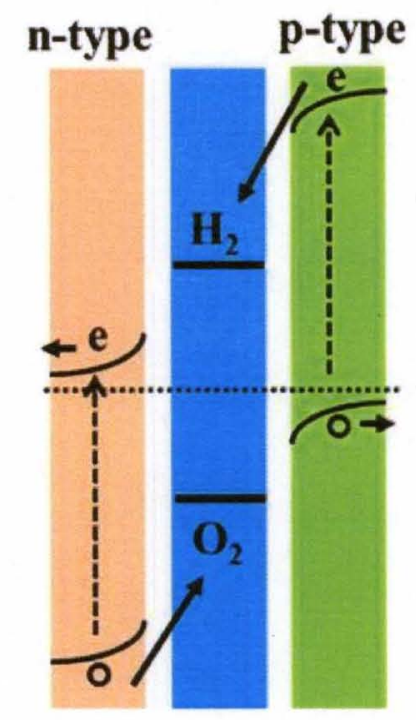

(b)

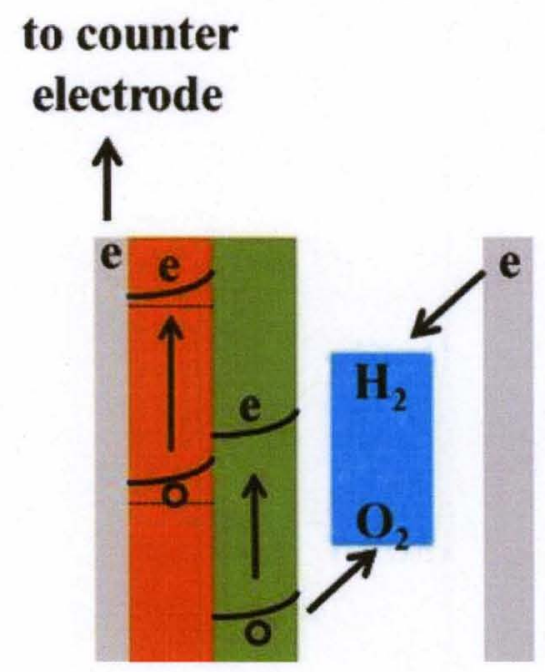

Figure 1.4 Dual material PEC cell designs a) Schematic of a PEC cell with a p-type and n-type electrode. Minority charges from each electrode drive the two water splitting reactions. Materials must be selected such that the minority band edge straddles one of the redox potentials. b) A stacked electrode where the materials have to be selected with proper conductivity type and band alignment such that majority carriers are transported without barriers to the back contact and minority carriers are transported to the surface.

Alternatively, as shown in the figure $1.4 \mathrm{~b}$, a stacked structure can also be developed. Here, material selection is very critical as misalignment can cause recombination at the interface as seen in the $\mathrm{Si} / \mathrm{TiO}_{2}$ core shell nanowire structure ${ }^{41}$. The band edges have to be matched such that the majority carriers flow to the back contact and minority carriers flow to the surface. 
A similar band gap tailoring can be achieved in GaNSb system and it is our other system of interest. Composition based properties will be estimated theoretically to determine the composition for the required band gap of $2 \mathrm{eV}$ and the band structure will be calculated to check for straddling. GaSbN can be one of the complementary materials for dual material system with InGaN if the band edges are in the right position. This is a further motivation to estimate the electronic structure of $\mathrm{GaSbN}$ accurately.

\subsection{Interest in nanowires}

Nanowires are the frontiers of modern technology. Apart from directly evident advantages of huge surface area enhancement for the same device area compared to thin films, nanowires also offer enhanced single crystalline charge transport pathways. Figure 1.4 shows the schematic comparing nanowire and planar architecture. For light absorption, penetration depth is defined as the minimum thickness of film required for carrier generation which is a direct derivative of the absorption coefficient of the material. Also, as seen in figure 1.5 , in thin films, the generated carrier has to travel enormous lengths much larger than the carrier diffusion length (before recombination) which leads to losses due to recombination. In nanowires, light can be absorbed along the length providing the absorption and the generated carriers can be effectively separated as the minority carriers have to travel very small distances (radius of the nanowire) to reach the surface and perform the reactions while the majority charge carriers can be transported to the back contact through the single crystalline nanowire core. It has 
actually been estimated that device performance improves when nanowires are used instead of films ${ }^{42}$.

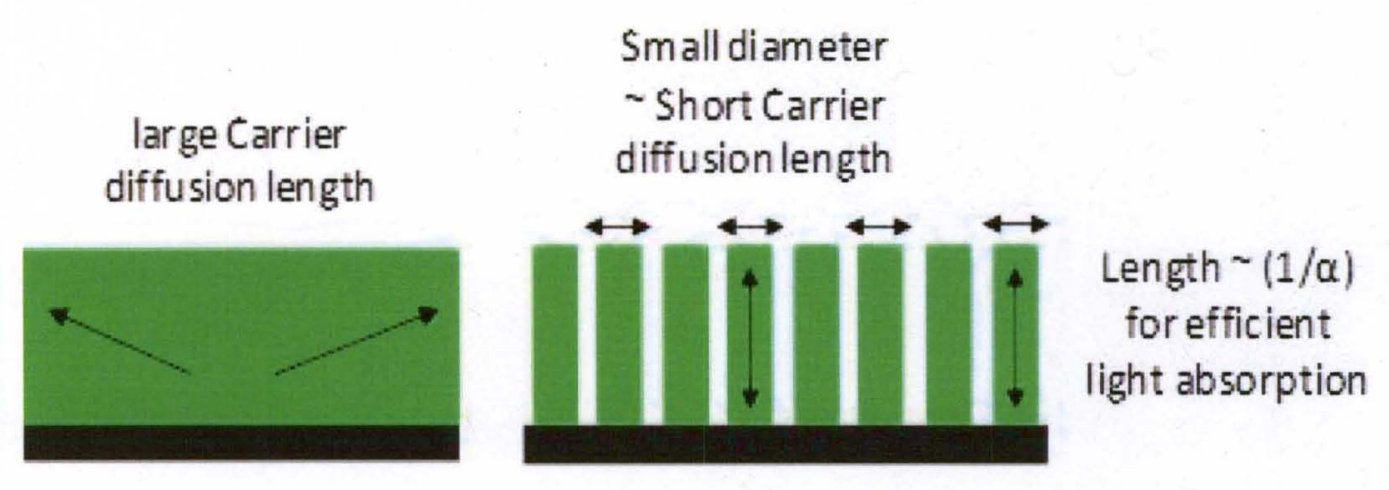

Figure 1.5 Schematic illustrating the advantage of nanowire architecture over planar thin film in terms of increased surface area available for reactions and smaller length scale for minority carriers to reach the surface (reducing recombination).

Another issue that makes nanowires more interesting is the problem in synthesis of single crystalline ternary alloys. InGaN has been known to be an unstable system ${ }^{43}$ at the required high indium content (for $2 \mathrm{eV}$ band gap) and synthesis of thick films has always been observed to result in phase segregation ${ }^{44}$ and formation of dislocations ${ }^{45}$. Synthesis of InGaN on heteroepitaxial substrates is always hampered by lattice mismatch. This leads to build up of strain energy in the growing film that is a function of the thickness and the lattice mismatch with the substrate. Critical thickness is the thickness of the film where the energy in the film increases to the point where it becomes favorable to segregate into indium rich and gallium rich phases or growth of dislocations. A material of lattice constant $\mathrm{a}_{\mathrm{f}}$ growing on a substrate of lattice constant $\mathrm{a}_{\mathrm{s}}$, the misfit is given by equation 1.1 


$$
f=\frac{a_{s}-a_{f}}{a_{s}}
$$

The elastic strain energy per unit surface of a film of thickness " $t$ " of a material with Poisson's ratio " $v$ " and shear modulus " $\mu$ " is given as 46

$$
E=2 \mu f^{2} t\left(\frac{1+\nu}{1-\nu}\right)
$$

As the thickness of the film increases, the strain energy also increases according to equation 1.2. The formation of indium rich quantum $\operatorname{dots}^{47}$ and phase segregation is a common feature in InGaN thin film growth ${ }^{48,49}$. Nanowires have been shown to have efficient strain relaxing properties. It has also been calculated that the critical thickness for phase segregation to occur is much larger in the case of nanowires than thin films ${ }^{50}$ which is a testament to the enhanced strain relaxing properties of nanowires ${ }^{51}$. The strain induced by the shell can be shared by the small diameter core which could act as a semirigid substrate. Very recently, silicon ${ }^{52}$, iron oxide ${ }^{53}$ and branched ${ }^{54} /$ Core shell $\mathrm{TiO}_{2} / \mathrm{Si}^{41}$ nanowires have been studied for their light harvesting and PEC water splitting performance and have shown promising performance. Shell growth on nanowires can also be used to passivate surface states to improve charge optical properties ${ }^{55}$.

Synthesis of InGaN nanowires has only been reported only via halide $\mathrm{CVD}^{56,57}$ and others were limited to a maximum indium content of $30 \%$ which is not suitable for PEC water splitting ${ }^{58}$. Growth of InGaN on nanowires has also been attempted for quantum wells ${ }^{59,60}(<10 \mathrm{~nm})$ which are too thin for light absorption as well as layers on thick GaN nanowires ${ }^{61}$ (nanowire diameter $>100 \mathrm{~nm}$ ) where phase segregation was observed. The regime of growing InGaN films on thin $\mathrm{GaN}$ nanowires has not been investigated. 


\subsection{Our approach}

Synthesis of thick InGaN alloy films on planar substrates has been a problem due to phase segregation. Nanowires on the other hand have inherent advantages in surface area and charge transport due to their aspect ratio and have been shown to have excellent strain relaxation properties. Our study is geared towards studying the heteroepitaxial growth process of ternary $\mathrm{InGaN}$ alloys on $\mathrm{GaN}$ nanowires. We propose that nanowires of reasonably small diameter would act as compliant semi-rigid substrates that would accommodate a part of the stress on the growing InGaN film, helping in mitigating phase segregation.

Towards this objective, the first step is the controlled synthesis of $\mathrm{GaN}$ nanowires on various substrates. Device applications require the charge carriers to move from the wires to the counter electrode via the back contact. Growing the nanowires directly on the metal substrates allows for the creation of the back contact without the need for post synthesis transfer of the nanowires. Hence, GaN nanowires will be synthesized on metallic as well as semiconductor substrates. Mechanism of GaN nanowire growth on various substrates and the subsequent orientation control will be examined allowing for direct synthesis of $\mathrm{GaN}$ on various substrates. This study will not only allow for the synthesis of phase stable InGaN alloys for evaluation of InGaN as a PEC material but also provides a framework for the development of nanowire based architectures while using nanowires as strain relaxing substrates. Also, the orientation dependence of InGaN growth on the underlying GaN nanowire substrates will be studied. 
Synthesized of InGaN layers on GaN nanowire substrates will be conducted in a custom built MOCVD reactor. Optimum reactor and process conditions will be modeled to achieve the best growth conditions. Comprehensive analysis of the growth mechanism and properties of the synthesized alloy films will be performed to understand the true nature of heteroepitaxial growth on nanowire substrate. This is where the idea of nanowires acting as strain relaxing substrates will be put to test.

As shown in the schematic in figure 1.6a, the ideal device structure consists of vertical arrays of $\mathrm{GaN}$ nanowires on metal substrate. Such as architecture would not only provide a direct electrode structure but aid in a uniform growth of InGaN layers on the nanowires. The shell material can be doped $\mathrm{p}$ type if needed. Also, as shown in figure $1.6 \mathrm{~b}$, a protective layer can also be coated onto the InGaN layer and the metal substrate to protect the semiconductor from degradation as well as the metal from stray reactions with the electrolyte. The thin layer can be coated with ALD (atomic layer deposition) would prevent corrosion but allow charge carriers to reach the surface via tunneling.

Apart from synthesis of InGaN, a new ternary system GaSbN (with dilute antimony alloying) would be investigated theoretically for its composition dependent band gap and band edge positions. Knowledge of the composition required to achieve a $2 \mathrm{eV}$ band gap will allow the design of proper synthesis experiments. 
(a)

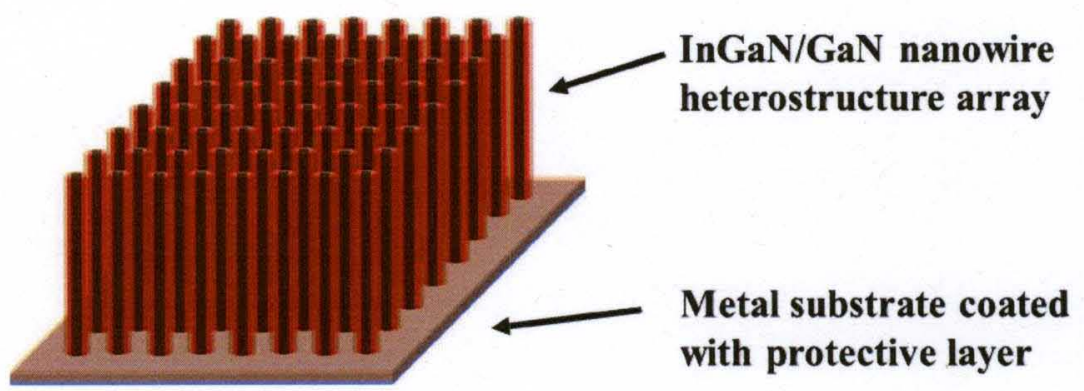

(b)

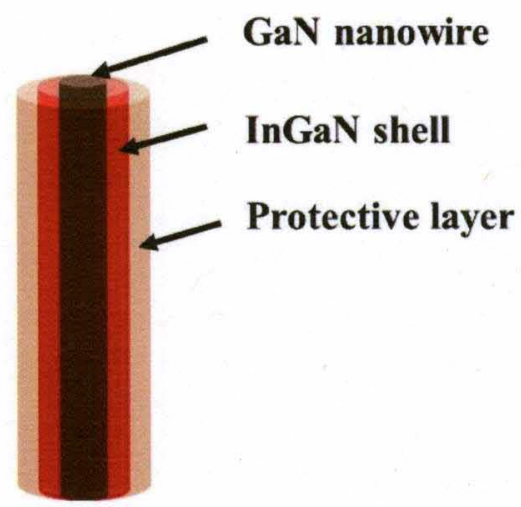

Figure 1.6 a) Schematic of the ideal nanowire growth pattern on metal substrates. The vertical arrays would help in a uniform growth of the InGaN layers. b) The central GaN nanowire core would be coated with a single crystalline shell of InGaN of the desired band gap (composition). Finally, a protective coating can be grown on the $\mathrm{InGaN}$ to protect from any corrosion. 


\subsection{Organization of thesis}

The thesis is divided into nine chapters. The first chapter is an introduction to the need to the study. It also includes the rationale for selection of material systems ( $\mathrm{InGaN}$ and $\mathrm{GaSbN}$ ) and our interest in nanowire based architectures. The second chapter includes a thorough literature survey on the current state of the art in PEC water splitting, the status of the materials search, an in depth look at InGaN as a material system, the issues with $\mathrm{InGaN}$ and work done on heteroepitaxial film growth and on nanowire substrates. The third chapter contains experimental procedures for the synthesis and characterization performed. Fourth chapter has the details on the reactor design, flow and results on reaction modeling.

The fifth chapter has the results from all the nanowire synthesis experiments and studies on the growth mechanism and control of nanowire growth mode. The sixth chapter includes the results from the InGaN synthesis experiments. Results from the band gap, composition and structural characterization are included to show the composition controlled synthesis of InGaN alloys. A discussion on the observed dependence of the InGaN growth on the nanowire orientation is also presented.

The sixth chapter contains the procedure and results of the theoretical estimation of the electronic properties of $\mathrm{GaSbN}$ alloy system and its applicability towards spontaneous PEC water splitting. Conclusions from the study are included in chapter eight and recommendations are presented in chapter nine. 


\section{CHAPTER 2}

\section{BACKGROUND}

\subsection{Introduction}

This chapter includes a review on the current state of the art in materials search for PEC water splitting. It also includes a detailed discussion on InGaN as a material system and the associated issues for PEC water splitting. We will also discuss the work done on nanowire based architectures for the mitigation of phase segregation.

\subsection{Review of PEC water splitting materials}

\subsubsection{GaN based systems}

As discussed above, our materials of interest are $\mathrm{InGaN}$ and $\mathrm{GaSbN}$. However, $\mathrm{GaN}$ and other $\mathrm{GaN}$ based systems have also been studied for their water splitting properties. The large band gap of $\mathrm{GaN}$ makes the material suitable for water splitting only under UV light which is very small part of the solar spectrum. Hence, the little interest in direct water splitting using GaN. p-GaN has been shown to have good stability 
in aqueous solutions. Hence, alloys based on $\mathrm{GaN}$ are being increasingly studied. GaPN and GaAsPN were evaluated for their PEC performance ${ }^{35}$ but these materials have the conduction band edges positive to hydrogen evolution potential requiring external bias.

Recently, $\mathrm{GaN}: \mathrm{ZnO}^{62,63}$ ternary system has shown some promising results. Here, the low performance of the alloy was attributed to the synthesis in powder form which enhances recombination. Also, the synthesis is a very slow and long process. Further characterization of the alloy is needed to study its stability and the actual composition suitable for water splitting.

Very limited work has been done on InGaN alloys for water splitting. P-InGaN electrodes (with indium content $<22 \%$ ) were shown to have better performance than $\mathrm{p}-\mathrm{GaN}^{64}$ but are still not in the right band gap regions. $\mathrm{HBr}$ was used as the electrolyte in this study which is not safe. In another study, InGaN films of $20 \%$ and $40 \%$ indium were tested for their PEC performance. The low indium content film did not have any phase segregation while the $40 \%$ indium containing film shows phase segregation from the shoulders in the PL spectrum.

\subsubsection{Other systems}

As discussed earlier, many material systems have been studied for their PEC water splitting properties as single material systems as well as in tandem cell or compensated architectures. 
Materials like CdSe and $\mathrm{Fe}_{2} \mathrm{O}_{3}$ are close to straddling and have the right band gap. CdSe has the band gap but is very prone to photocorrosion. It was also shown to not catalyze the reaction ${ }^{65}$. However, using sacrificial electro donors makes CdSe nanoribbons a usable electrode ${ }^{66}$. This result shows that size constraint could be a useful tool in improving the material performance. Iron oxide films have poor charge transport properties $^{67}$ as the material is a known mott insulator. It has been shown that compared to the penetration depth of $118 \mathrm{~nm}$ at $500 \mathrm{~nm}^{68}$ the hole diffusion length has been measured to be $2-4 \mathrm{~nm}^{69}$ which means that a $100 \mathrm{~nm}$ thick film is required for the light to penetrate and generate charge carriers. However, the generated holes never make it out to the surface and are lost due to recombination, destroying the efficiency of the material to act as a PEC water splitting semiconductor. As a single material system, CdSe might not be very interesting. However, CdSe quantum dots in conjunction with other host materials have been employed to improve the water splitting efficiency of the host material. Here CdSe acts as a sensitizer absorbing sunlight and injecting electrons into the host material. CdSe quantum dot sensitization has been shown to improve the water splitting efficiency in $\mathrm{ZnO}$ nanorods $\mathrm{s}^{70}$ nanotubes ${ }^{71}$ as well as n-doped $\mathrm{TiO}_{2}$ nanorod arrays ${ }^{72}$. Au loading into n-doped TiO2 along with CdSe sensitization showed increased enhancement over the visible spectrum.

A new approach using a double sided ${ }^{73} \mathrm{CdS}$ and $\mathrm{CdSe}$ coated $\mathrm{ZnO}$ nanorod arrays showed improved performance under white light. Multiple material electrode made with $\mathrm{CdSe}$ and $\mathrm{CoS}$ was studied for unbiased water splitting ${ }^{74}$. Figure 2.5 shows the setup employed for the double sided $\mathrm{CdSe}$ and $\mathrm{CdS}$ coated $\mathrm{ZnO}$ nanorod arrays. Here, the two materials act as electron injectors while absorbing light in the visible region with the $\mathrm{ZnO}$ 
scaffold absorbing in the UV and acting as a charge transport pathway for the injected electrons. Enhancement is observed in terms of light absorption range and this provides a template to improve the performance of other systems as well. However, as shown in the figure 2.1, the band edge positions have to be right to have an efficient injection as well as prevent recombination. Furthermore, the stability of such systems has to be determined for practical applications.

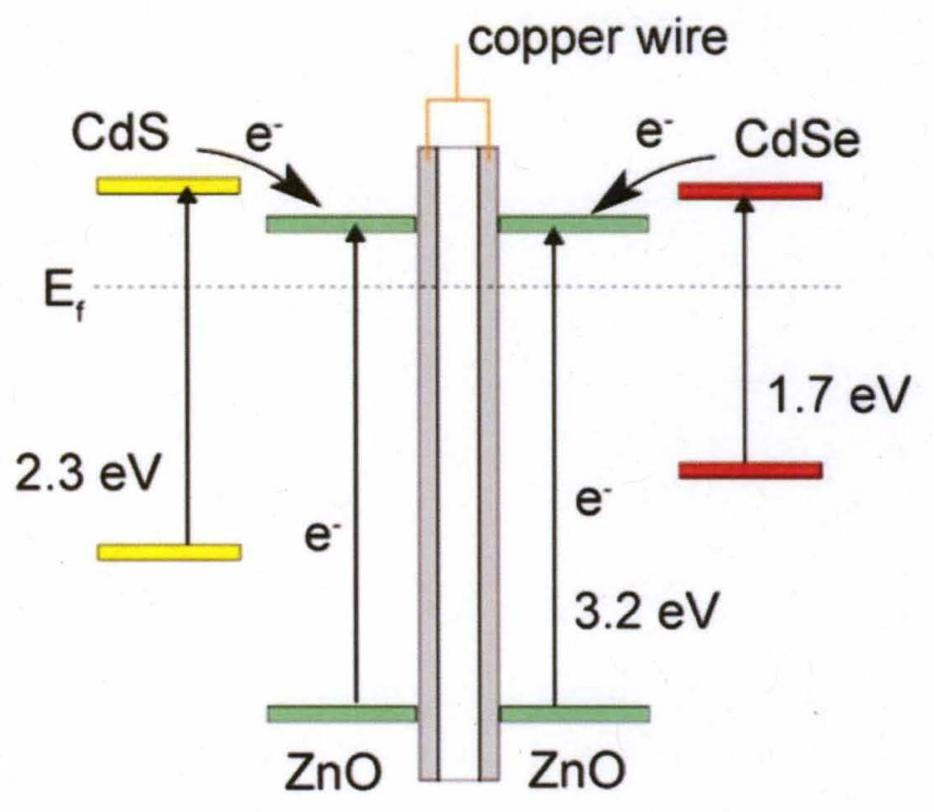

Figure 2.1 Schematic of the band alignment of the double sided CdSe and CdS coating on the $\mathrm{ZnO}$ nanowire arrays. The two materials act as visible light absorbers and inject electrons into the $\mathrm{ZnO}$ scaffold ${ }^{73}$. Similar approach of sensitization has also been studied for $\mathrm{ZnSe}$ on $\mathrm{ZnO}$ nanostructures ${ }^{75}$ which showed much improved performance under visible light illumination. 
Recently, there has been a lot of work on improving the photoactivity of hematite that has shown great promise and improved results ${ }^{76}$. Very interesting is the fact that most of them employ nanowire based structures that help improve the charge transport properties. The material is modified by changing the morphology or doping or a combination of both to improve the PEC water splitting efficiency. Nanostructured hematite films prepared by the thermal oxidation of DC arc sputter deposited iron films at $255 \mathrm{C}$ resulted in improved photoactivity. Various other approaches such as doping with aluminum ${ }^{77}$, silicon $^{78}, \operatorname{tin}^{79}$ and platinum ${ }^{80}$ and surface passivation with alumina ${ }^{81}$. All these approaches are with one material system.

Similar to the sensitization of $\mathrm{CdSe}$ and $\mathrm{ZnSe}$, dual material approach has also been employed with hematite systems. $\mathrm{WO}_{3}-\mathrm{Fe}_{2} \mathrm{O}_{3}$ host-scaffold method showed improved visible light absorption ${ }^{67}$ than bare $\mathrm{Fe}_{2} \mathrm{O}_{3}$. Nanostructured $\mathrm{WO}_{3} / \mathrm{BiVO}_{4}$ electrodes showed improved light absorption due to enhanced visible absorption from $\mathrm{BiVO}_{4}$ and charge separation at the interface of the two materials. Wide band gap semiconductors such as $\mathrm{GaN}, \mathrm{TiO} 2$ and $\mathrm{ZnO}$ have band edge positions that straddle the water redox potentials but the band gap is too high that makes the material inefficient absorber limiting the carrier generation. Doping with nitrogen in the case of $\mathrm{ZnO}^{82}$, $\mathrm{TiO}_{2}{ }^{83}$ and $\mathrm{WO}_{3}{ }^{84}$ as well as phase transformation into $\mathrm{W}_{2} \mathrm{~N}$ nanowire arrays has been attempted to move the absorption into the visible region with some success. Sensitization and electron injection using a lower band gap material has shown some improvement but the spontaneous visible water splitting still remains elusive. Also, stability in such cases needs to be examined thoroughly as interfaces can create new pathways for recombination. 
A screening approach has been recently proposed for metal oxide systems that could have potential implications based on their being mott insulators or not ${ }^{85}$. This includes two very necessary approaches to make the material work as a stable PEC photoanode. From the standpoint of stability, hydrogen evolution at the material surface would help against corrosion by oxidation. This however requires the material to be ptype when the minority carriers (electrons) would drive the hydrogen evolution reaction. Most of the oxides have large band gaps and are inherently n-type that leads to oxygen evolution at the surface. Small band gap materials can still have stability issues in aqueous solutions. The most important point here is that transition metal oxides have a small band gap and their valence bands are much lower than the oxygen evolution potential. This makes straddling the hydrogen potential by conduction band almost impossible in most materials which has indeed been observed. Doping or alloying has been attempted to be a way to solve this problem but without much success. Localization of states at the band edges finally controls the charge transport and is heavily influenced by the dopant or alloying species. In many of the transition metal oxides, both the bands are localized due to the metal cations making them "Mott-Hubbard" insulators. This argument is applicable for binary as well as ternary metal oxides.

We have seen that binary materials are not suitable for direct water splitting and have not shown unbiased water splitting even with many configurations. This combined with the fact that ternary semiconductors are solid solutions with composition dependent band gap makes these materials interesting. Although many ternary alloys can be synthesized with band gaps in the rage useful for PEC water splitting, only a few have been tested and so far none has band edges straddling the redox potential. 
Most of the work has been focused towards the non-oxide ternary semiconductors and $\mathrm{InGaN}$ has been the most studied material in terms of growth and properties. The interest in InGaN started with LEDs ${ }^{86,87}$ but it has started to gather attention for application in solar cells ${ }^{88,89}$ and more recently water splitting ${ }^{90,91}$. Other ternary system of interest is $\mathrm{GaInP}_{2}{ }^{92,93}$ and $\mathrm{GaAsPN}^{35}$. Both have issues with valence band being positive of the oxygen evolution potential making unbiased water splitting possible. A water splitting system with a PV cell in tandem using GaInP2 electrode resulted in the highest recorded water splitting efficiency of $12.4 \%{ }^{38}$. p-GaInP $P_{2}$ was also used as a photocathode in conjunction with a nanostructured hematite and $\mathrm{WO}_{3}$ photoanode but the device was very limited in current generation and efficiency even at high illuminations of $1 \mathrm{~W} / \mathrm{cm}^{2}$ possible due to hematite ${ }^{93}$. Very recently, a GaN: $\mathrm{ZnO}$ solid solution ${ }^{62,94}$ has been shown to be a promising material system for water splitting. The efficiency has been very low and further investigation is needed on study of the band edge positions are necessary.

Hence there is a need for continued search for materials for water splitting. It is clear that none of the single band gap systems have efficiencies close to that of a PV assisted system ${ }^{37}$. However, the synthesis of such band edge engineered systems is a challenge and expensive which is a major drawback.

\subsection{InGaN: introduction to the material}

InGaN is a logical material system to be investigated as it is a ternary derivative of $\mathrm{GaN}$ and $\mathrm{InN}$. GaN is stable in aqueous solutions ${ }^{84}$ and its band edges straddle water 
splitting potentials ${ }^{39}$. The interest in InGaN arises from the composition dependent band gap tunable from $3.4 \mathrm{eV}(\mathrm{GaN})$ to $0.7 \mathrm{eV}(\mathrm{InN})$. Table 2.1 lists the basic structural and electronic properties of $\mathrm{GaN}$ and $\mathrm{InN}$.

Table 2.1 Basic properties of interest of $\mathrm{GaN}^{95}$ and $\mathrm{InN}$. InGaN has a composition dependent variation for the structural and optical properties.

\begin{tabular}{|ccc|}
\hline Property & GaN & InN \\
\hline Crystal structure & wurtzite & Wurtzite \\
\hline Lattice constant a (A) & 3.189 & 3.533 \\
\hline Lattice constant c (A) & 5.178 & 5.693 \\
\hline $\begin{array}{c}\text { Synthesis } \\
\text { temperature (C) }\end{array}$ & $850-1000$ & $500-700$ \\
\hline Band gap (eV) & 3.4 & 0.8 \\
\hline
\end{tabular}

\subsubsection{InGaN Synthesis}

\section{Methods}

InGaN alloy is a solid solution all the way from $\mathrm{GaN}$ to $\mathrm{InN}$. Hence, the various process conditions (temperature, pressure, flow rate) are used to control the composition of the alloy. Various techniques such as thermal $\mathrm{CVD}^{96,97,98}, \mathrm{HVPE}^{58,56,57,99}$,

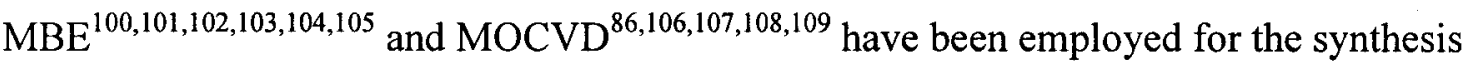
of InGaN films and nanowires. The interest in InGaN is mostly due to its optical properties mainly as quantum well structures for LEDs that requires very thin layers $(<$ $10 \mathrm{~nm}$ ). Very recently, InGaN is being increasingly investigated for its light absorption 
properties for applications such as solar cells and water splitting. These applications require thick (100-200 nm) single crystalline InGaN films for efficient light absorption. Thick InGaN alloy films and films with high indium content have always been marred by phase segregation ${ }^{110}$.

Each of the synthesis techniques has its own set of advantages and disadvantages. Thermal CVD using Gallium, indium and ammonia is very inexpensive but the problem is in the independent control of indium and gallium flux. This can be done using independent heating of the materials but control is still not exact. Another route in thermal CVD is to use GaN and InN powders which still need independent heating. Commonly, a dual zone tube furnace is used where the precursors are heated upstream and generated vapors travel downstream to a colder zone to react or deposit onto a substrate. Apart from temperature and pressure, position of the substrate is critical in such reactors.

HVPE employs gallium chloride and indium chloride along with ammonia for the synthesis. HVPE is the only method that has been reported to lead to synthesis of InGaN nanowires with the complete range of Indium compositions ${ }^{56,57}$. The fluxes were controlled independently but the issue here is the chloride species that can be detrimental to the metal parts of the reactor. The setup is similar to that in CVD with the exception that the chloride precursors can be heated in the tube or a carrier gas can be bubbled to carry vapors independently into the reactor.

MBE utilizes metal Knudsen cells for controlled supply of material flux onto a substrate. The substrate is placed at the center of a sphere with the material cells arranged so that the flux is focused at the center. The system is run at very low pressures $\left(10^{-7}\right.$ 
Torr) and at a very small growth rate that leads to the formation of single crystals of very high quality. However, the MBE apparatus is very expensive and the growth rates add to the operating costs. Also, the substrate size is limited and uniformity over large area is an issue.

MOCVD is the upcoming method for the synthesis of good quality single crystalline materials. Here, metalorganic precursors (TMGa/TEGa and TMIn/TEIn) are supplied into the chamber in vapor phase. This allows for a very precise control over the material flux. MOCVD has the advantage of high growth rate and excellent flux control. However, the precursors are commonly pyrophoric which makes elaborate safety mechanisms necessary making the system expensive. The supply of precursors in vapor phase makes this a very customizable process. Various reactor shapes have been employed and modeled to improve uniformity of growth and maximize material use. Typically, vertical flow is used for increased uniformity. A showerhead is used to distribute the precursors over the entire substrate area for uniform precursor distribution. However, horizontal quartz tube reactors with an external IR heating source are also very common. Also, modeling of various reactor configurations has shown that the uniformity and growth rates are very specific functions of the reactor geometry which means that each type of reactor has to be tuned for the best growth parameters.

\section{Growth mechanism}

MBE and MOCVD of InGaN are quite different not only in terms of precursors employed but also in the nature of the growth process. Figure 2.2 shows the basic mechanisms of MBE and MOCVD reactions indicating the differences. In MBE, as shown in figure 2.2a, the group III and group V atoms impinge on the substrate where 
they react. This is a purely kinetic controlled process at the very low pressures. Reactions occur only at the surface which makes the process clean without any parasitic reactions. Such process and the low pressures allows for an accurate thickness monitoring using RHEED.

In MOCVD however, as shown in figure 2.2b, Trimethylallium/Triethylgallium, Trimethylindium/Triethylindium are the most commonly employed precursors. The species are not pure group III atoms but organic groups linked to gallium and indium atoms. The feature of these precursors is that partial pyrolysis occurs even at low temperatures along with parasitic reactions with the carrier gases away from the substrate in the reactor volume. The reactions, affected by the process conditions, have been observed to interfere in the growth of clean uniform surfaces via the formation of particles in the gas phase ${ }^{111}$. Suppression of such reactions is critical especially in the growth of single crystalline films. This can be obtained by pulsed precursor injection as well as having isolated precursor injection lines into the reactor and having the substrate close to the injection ports. 
(a)
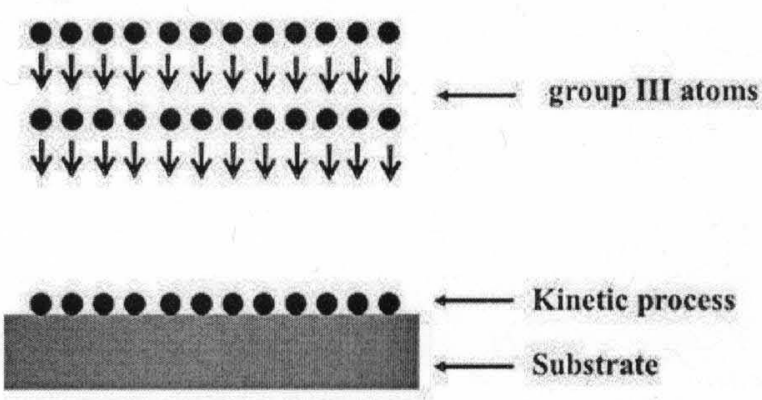

(b)

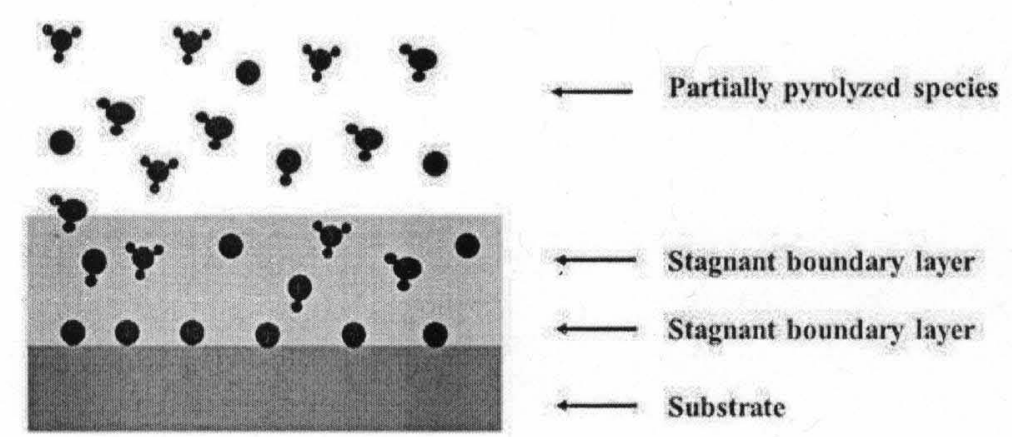

Figure 2.2 Schematic of the reaction processes in a) MBE and b) MOCVD growth. In MBE, the pure metal atoms impinge onto the substrate and react on the substrate at high temperature to form the product. b) In MOCVD, the reactive precursors start reactions and partial pyrolysis form adducts and particles in gas phase. The surface reactions are carried out by adducts (adatoms) that are not pure metal atoms which then have different reaction properties and diffusion rates than in MBE.

As shown in figure $2.2 \mathrm{~b}$, the MOCVD process is not a simple kinetic process. The process shifts from kinetically controlled where the growth rate is directly dependent on the reaction rate to diffusion controlled where the kinetics are not the limiting step but the diffusion of species from the reactor volume to the substrate through the boundary 
layer. This offers the most control over the growth process as species diffusion is influenced and hence controlled by the precursor flux. Further increase in temperature moves the system to a thermodynamically controlled regime where the adatom growth rate is affected by the increased mobility of adatoms and increased detachment of adatoms at the high temperatures. The plot of growth rate vs. temperature shown in figure 2.3 illustrates the process. The best growth zone is at the highest temperature in the diffusion limited region where the growth rate is high and crystal quality is high at the high temperature.

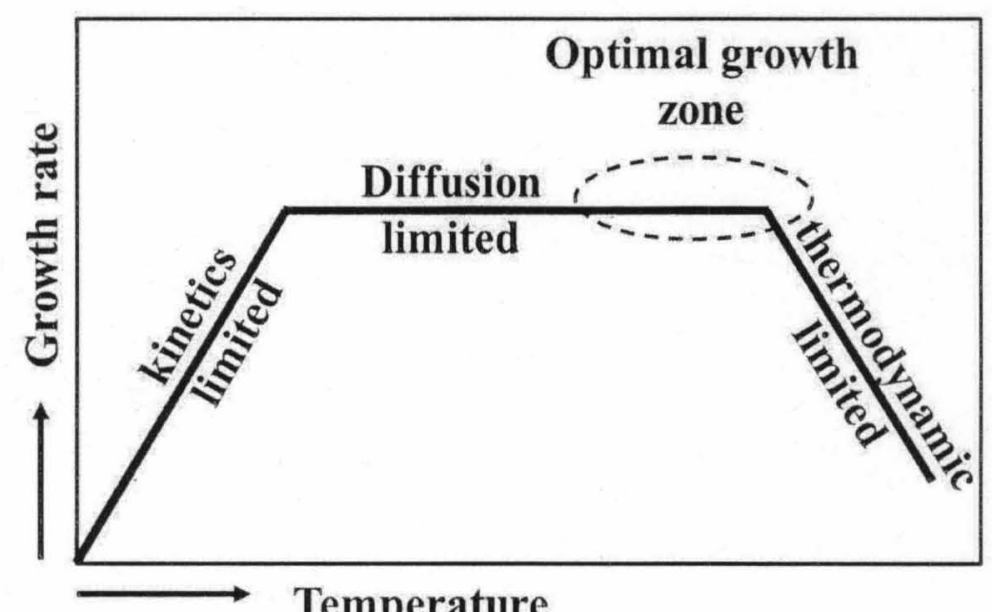

Figure 2.3 Generic schematic of the MOCVD growth process showing the different growth regimes.

Partial pyrolysis leads to the formation of numerous intermediate (adduct) gas and surface species during MOCVD growth. InGaN due to the presence of indium and gallium species requires elaborate detection of species as numerous inter species reactions and adduct formations can occur among the indium and gallium adducts. GaN 
has been studied extensively for the reactions with some work on InN as well. Table 2.2 lists the vapor phase and surface reactions leading to the formation of $\mathrm{GaN}$.

Table 2.2 Gas phase and surface reactions for MOCVD growth of MOCVD ${ }^{112}$.

\begin{tabular}{|c|}
\hline Reactions \\
\hline Gas phase reactions \\
\hline $\mathrm{TMG}+\mathrm{NH}_{3} \rightarrow \mathrm{TMG}: \mathrm{NH}_{3}$ \\
\hline $\mathrm{TMG}: \mathrm{NH}_{3} \rightarrow \mathrm{TMG}+\mathrm{NH}_{3}$ \\
\hline TMG: $\mathrm{NH}_{3} \rightarrow \mathrm{DMG}: \mathrm{NH}_{2}+\mathrm{CH}_{4}$ \\
\hline $3 \mathrm{DMG}: \mathrm{NH}_{2} \rightarrow \mathrm{TGNCY}$ \\
\hline Surface reactions \\
\hline $\mathrm{TMG}: \mathrm{NH}_{3}+2 \mathrm{~S} \rightarrow \mathrm{GaN}(\mathrm{s})+3 \mathrm{CH}$ \\
\hline DMG: $\mathrm{NH}_{2}+2 \mathrm{~S} \rightarrow \mathrm{GaN}(\mathrm{s})+2 \mathrm{CH}$ \\
\hline $\mathrm{TGNCY}+6 \mathrm{~S} \rightarrow 3 \mathrm{GaN}(\mathrm{s})+6 \mathrm{CH}_{4}$ \\
\hline
\end{tabular}

The reactions for the formation of the adducts TMG:NH3 and DMG:NH2 are spontaneous which is measured and hence shows that adduct formation is indeed the dominant mechanism in MOCVD process.

\section{Role of process parameters on InGaN MOCVD growth}

The adatoms form on the substrate and the process conditions define the attachment/detachment rates which determines the final growth ${ }^{110}$. InGaN synthesis is usually performed at temperatures more than $\mathrm{InN}$ and less than $\mathrm{GaN}$ synthesis temperatures. At these conditions, $\mathrm{InN}$ has a higher vapor pressure than $\mathrm{GaN}$ causing 
reduced indium incorporation ${ }^{113}$. This process is influenced by the process parameters (temperature, precursor III/V ratio and pressure).

Reaction temperature has a drastic influence on the MOCVD process. The effect increases for ternary systems. In the case of binary system, lower temperatures lead to the formation of structural defects as the adatom diffusion is limited at lower temperatures. This can also lead to the inclusion of impurities into the lattice. At high temperatures, there is increased desorption, larger diffusion of adatoms leading to better crystal quality. Also, nitrogen vacancies are observed due to increased nitrogen desorption. In the case of ternary systems such as InGaN, the effect is magnified. Indium species incorporation occurs at low temperatures while $\mathrm{GaN}$ reaction is a high temperature process. InGaN synthesis is at intermediate temperatures that are too high for $\mathrm{InN}$ and low for $\mathrm{GaN}$. This causes preferential desorption of indium adatoms leading to reduced indium content in the alloy ${ }^{114}$. Synthesis of $\operatorname{InGaN}$ at lower temperatures causes reduced gallium incorporation and degrades crystal quality by favoring defect formation due to reduced adatom diffusion. Also, cracking of ammonia is inefficient at low temperatures.

The III-V ratio also is critical in control of composition especially in ternary systems. Low III-V ratio causes increased indium incorporation ${ }^{115}$ but reduced surface mobility ${ }^{16,117}$ leading to the formation of structural defects. However, a high III-V ratio causes excess adatoms on the surface leading to decomposition and indium droplet formation without reaction as well as formation of nitrogen vacancies that is detrimental to the material properties ${ }^{118}$. Specific to the ternary system, the relative flux $\mathrm{In} / \mathrm{Ga}$ also needs to be controlled in conjunction with the other parameters. At high temperatures, a 
higher indium flux increases indium content but the incorporation plateaus at higher temperatures. This has also been observed in the case of HVPE growth of InGaN nanorods $^{119}$.

System pressure has been observed to influence the indium incorporation in the alloy film. At lower pressures, indium incorporation was observed to increase attributed to the increased diffusion into the boundary layer (shown in figure $2.2 \mathrm{~b}$ ) at the lower pressures ${ }^{120,121}$. This observation could be specific to species since the diffusion rates are material specific.

\subsubsection{Issues with InGaN}

\section{Thermodynamic Instability}

It has been estimated that InGaN alloy is thermodynamically unstable ${ }^{43}$ almost throughout the composition range and phase segregation is a mechanism to attain stability. Figure 2.4 is the phase diagram of the InGaN alloy system showing the extent of the immiscibility gap. The binodal curve is the liquidus line where any alloy composition in the canopy is likely to separate into gallium rich and indium rich compositions like to segregate into the compositions marked by the intersection of a horizontal line through the composition and the liquidus line. 


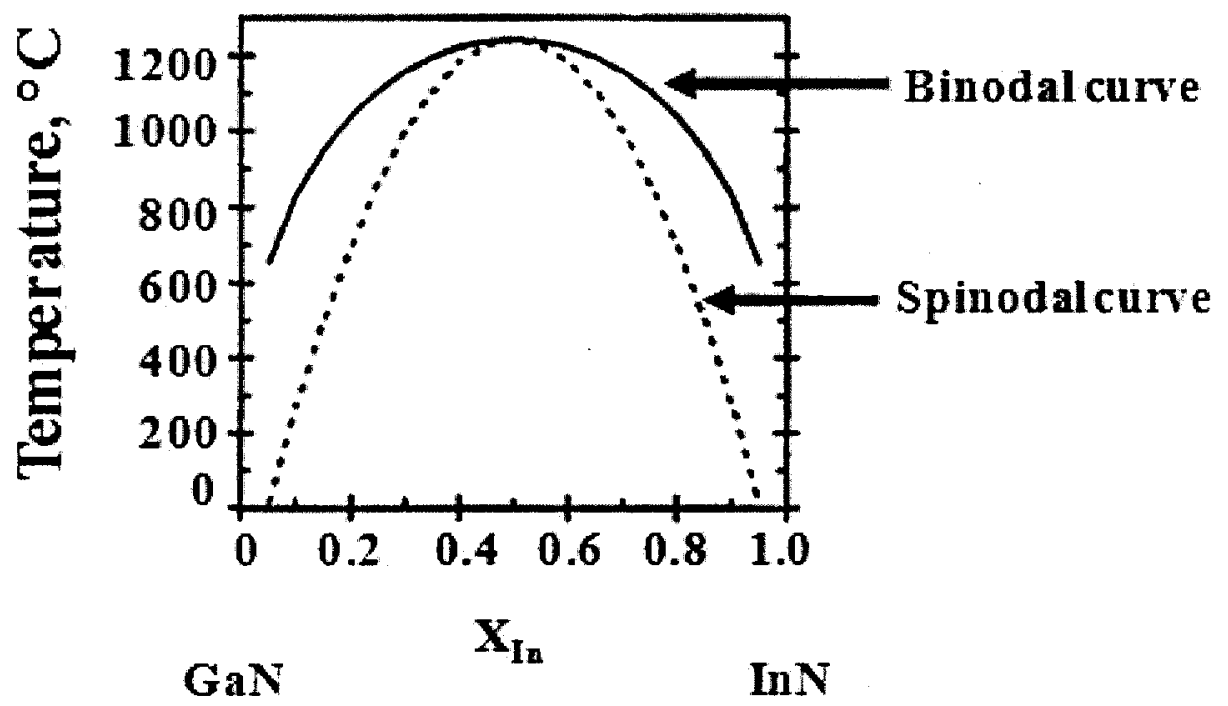

Figure 2.4 Phase diagram of the InGaN alloy system showing the immiscibility gap. The bimodal curve shows the instability of the alloy extending throughout the composition range. The dashed spinodal line is indicative of spontaneous segregation. Although narrower in spread, runs through the entire composition. Under the normal synthesis conditions, the alloys with $40-55 \%$ indium composition are thermodynamically unstable ${ }^{43}$.

The instability is attributed ${ }^{122}$ to the induced strain in the alloy owing to the size difference between the gallium and indium atoms ${ }^{123}$. The addition of larger indium atom (atomic radius $1.56 \mathrm{~A}^{124}$ ) into $\mathrm{GaN}$ lattice (gallium atomic radius $1.35 \mathrm{~A}^{125}$ ) causes distortion in the lattice creating stresses in the material and local disturbance in the electronic distribution. 


\section{Strained heteroepitaxial growth}

Apart from the inherent instability, the manner of growth of InGaN has also contributed to the observed phase segregation. Heteroepitaxial substrates such as $\mathrm{GaN}$ and sapphire were used for InGaN thin film growth due to the lack of native substrates. The lattice constant of $\mathrm{InGaN}$ is dependent on its composition spanning $\mathrm{GaN}$ to InN. Assuming a linear variation and growth on "c" plane substrates, the \% lattice mismatch for a $50 \%$ indium content alloy wrt GaN, InN and sapphire ${ }^{126}$ according to equation 1.1 is $4.9 \%, 4.5 \%$ and $16 \%$. Lattice mismatch of various substrates w.r.t a $50 \%$ indium containing InGaN alloy is shown in table 2.3. This emphasizes the fact that there is a very limited availability of native substrates for InGaN film growth. Surprisingly, silicon has similar lattice spacing and could be used as a native substrate. However, use of silicon as a substrate for $\mathrm{InGaN}$ was limited due to the opto-electronic device requirements. $\mathrm{ZnO}$ could be an interesting alternative as the material has a high band gap and InGaN can be used as an absorber layer on the material. The issue here is the expensive growth of single crystalline $\mathrm{ZnO}$ substrates. Similar synthesis issues are also encountered in the case of $\mathrm{LiAlO}_{2}$ and $\mathrm{MgO}$.

Strain relaxation can occur via a variety of mechanisms that primarily depend on the composition and film thickness. The epi layer can be pseudomorphically strained to accommodate the stress as a compliant film. The lattice constant of the epi layer in the growth plane gets distorted to match the substrate lattice parameter. However, the vertical lattice constant can relax in the growing film. This effect actually is magnified in the case of nanowires. Inter-diffusion of species at the interface is another mechanism of strain relaxation. The atoms in the epi layer rearrange and diffuse into the substrate, destroying 
the abruptness of the interface ${ }^{127}$. This phenomenon is very dependent on the diffusion coefficient of the epi layer atoms in the substrate which is material specific.

Table 2.3 Lattice mismatch of various substrates for $\operatorname{In}_{0.5} \mathrm{Ga}_{0.5} \mathrm{~N}$ alloy

\begin{tabular}{|cc|}
\hline Material & Lattice mismatch \% \\
\hline Gallium Nitride & 4.9 \\
\hline Sapphire & 16.3 \\
\hline Silicon Carbide & 9.8 \\
\hline Boron Nitride & 7.6 \\
\hline Germanium & 3.7 \\
\hline Silicon & 0.1 \\
\hline Zinc oxide & 4.5 \\
\hline LiAlO & 5.1 \\
\hline $\mathrm{MgO}$ & 8.8 \\
\hline
\end{tabular}

Stress build-up in thicker films or higher compositions can reach a value when the relaxation can occur via formation of defects/dislocations or by increasing the surface energy of the film. This can lead to roughening of the film surface. Such growth is not preferred for the growth of single crystalline films but has been used for the synthesis of quantum dots ${ }^{128}$. On the other hand, if the built up strain energy in the film reaches a critical value wherein the formation of misfit dislocations becomes energetically favorable, plastic strain relaxation occurs via the formation of misfit dislocations. 
In the case of InGaN, the interplay of two factors: indium content and film thickness compete in the stress build up and relaxation processes.

\section{Kinetic phase segregation}

InGaN alloy system is also affected by kinetic factors that are specific to the growth process. The difference in diffusion coefficients of the indium and gallium atoms causes intermixing in the bulk causing phase segregation. Different species diffuse randomly in the alloy forming random compositions which is a way to relax stress locally. This has been shown to be a temperature dependent process and can be controlled via low temperature growth ${ }^{129}$. At high temperatures indium atoms have a higher diffusion coefficient causing indium to move out of the bulk to the surface and desorb ${ }^{130}$.

\subsection{Review of heteroepitaxial growth of InGaN}

Heteroepitaxial growth of InGaN films has always been accompanied by phase segregation in various manifestations ${ }^{110}$ such as formation of defects and indium/gallium rich segregated regions indicative of strain relaxation. Phase segregation has been observed to depend on the thickness of layers and the composition. Figure 2.5 shows the dark field TEM image of InGaN layer on GaN substrate where the bark and bright contrast bands are indicative of phase segregation. The domains with larger indium content show stronger contrast variations which are evidence for larger phase segregation. 

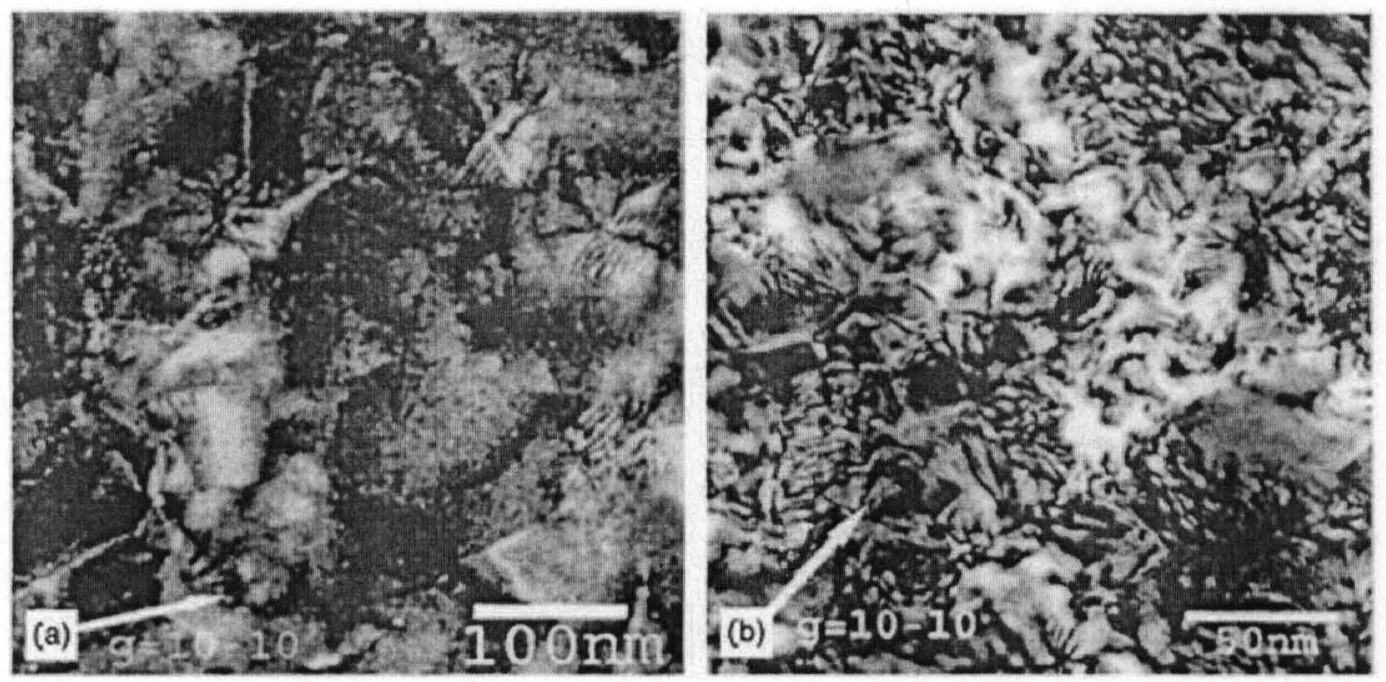

Figure 2.5 Dark field TEM images of InGaN layers on GaN with a) $12 \%$ indium showing a weak speckled contrast and b) $22 \%$ indium showing bands with higher contrast ${ }^{131}$.

Here, in layers close to the substrate, no contrast bands are observed. At low indium content $<12-14 \%$ there was no phase segregation. However, as the indium content increased, composition modulated regions form in the film ${ }^{131}$. Here, thin layers with high indium content $>12 \%$ also did not show any phase segregation (contrast in dark field images) possibly due to coherent strain that might inhibit phase segregation ${ }^{44}$. A composition pulling mechanism ${ }^{132}$ is proposed wherein initially indium incorporation is limited due to the strain. This reduces the driving force for phase separation. Also, the lower indium content in the initial layers increases the critical thickness reducing phase segregation. This seems to work for low indium content alloys.

Structural defects have been consistently observed in the growing InGaN epi layers. The defects sometimes carry over from the substrate aiding in strain relaxation but phase segregation is also favored at the defect lines creating local recombination centers. 
For thick InGaN layers, new dislocations can be generated in the film as a means to relax stress. This has been observed to be dependent on the growth parameters. As discussed earlier, higher temperatures and slower growth rates has been observed to form lower density of dislocations ${ }^{133}$ as the atoms get time to rearrange also increasing crystal quality. InGaN layers on $\mathrm{GaN}$ films have been observed to undergo strain relaxation via the formation of $\mathrm{V}$ shaped pits on the surface where the threading dislocations generated in the films open on the surface ${ }^{134}$. In cases where threading dislocations are absent, strain relaxation occurs via the formation of periodic arrays of misfit dislocations ${ }^{134}$. Stacking faults were also observed to lead to the formation of " $\mathrm{v}$ " shaped pits on InGaN surface ${ }^{135}$. The formation of "v" defects was also observed to depend upon the indium content and thickness of GaN film as shown in figure 2.6.

(a)

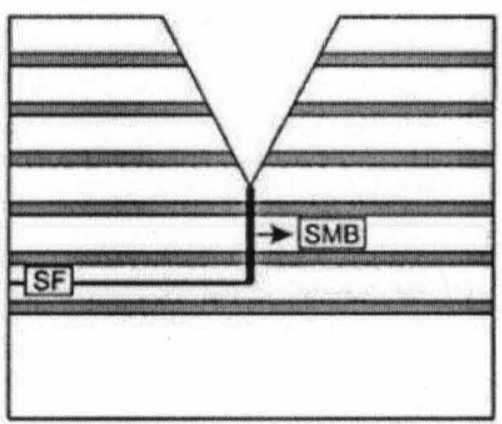

InGaN (b)

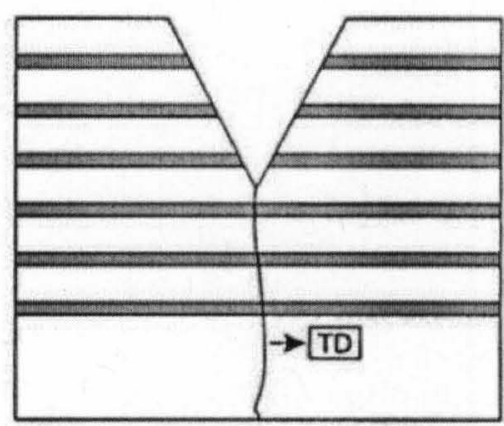

GaN

Figure 2.6 Schematic of the mechanism of the formation of "v" defects on InGaN quantum wells a) led by the stacking faults (at high indium content) and b) by the threading dislocations from the substrate into the epi layers ${ }^{135}$. 
Dislocations in InGaN layers have been also observed to not to extend to the surface but form pin holes at the interface attributed to the Matthews-Blakeslee model ${ }^{136}$. Dislocations in InGaN layers are the sites for phase segregation ${ }^{137}$ as they are energetically favorable sites than the rest of the lattice. Also, such dislocations were also shown to be non-radiative centers ${ }^{136}$ unlike the formation of quantum dots that act as radiative recombination sites. In some cases, strain has been shown to prevent phase segregation by limiting the segregation kinetics ${ }^{138,43}$.

Local plastic strain relaxation in InGaN films on GaN substrates was observed to occur via a "punch out" mechanism or the formation of dislocation loops ${ }^{139}$, as shown in the figure 2.7 . Noticeably, the films were $100 \mathrm{~nm}$ thick and the indium content only $11 \%$. Here, strain relaxation was observed to occur via the formation of misfit dislocations in the InGaN layer which along with the glide along the slip systems, lead to the punched out appearance shown in figure $2.7 \mathrm{a}$ where triangular regions are displaced due to the slip. In some cases, growth on bulk $\mathrm{GaN}$ substrates was also observed to lead to the formation of radial dislocation half loops as shown in figure $2.7 \mathrm{~b}$.

The presence of dislocations in the substrate was also observed to play an important role in the quality and growth of the growing InGaN film. Strain relaxation in the InGaN film on GaN occurred via the formation of misfit dislocations that propagated into the growing film which is also seen to depend on the indium content ${ }^{140}$. The number density and propagation distance of the dislocation loops was attributed to the increasing misfit stress again governed by the indium content of the film. Slip of the basal planes was also observed to lead to the formation of InGaN ledges on the GaN mesas. However, 
the distribution of species in the film was not reported which would demonstrate the role of plastic strain relaxation in controlling phase segregation.
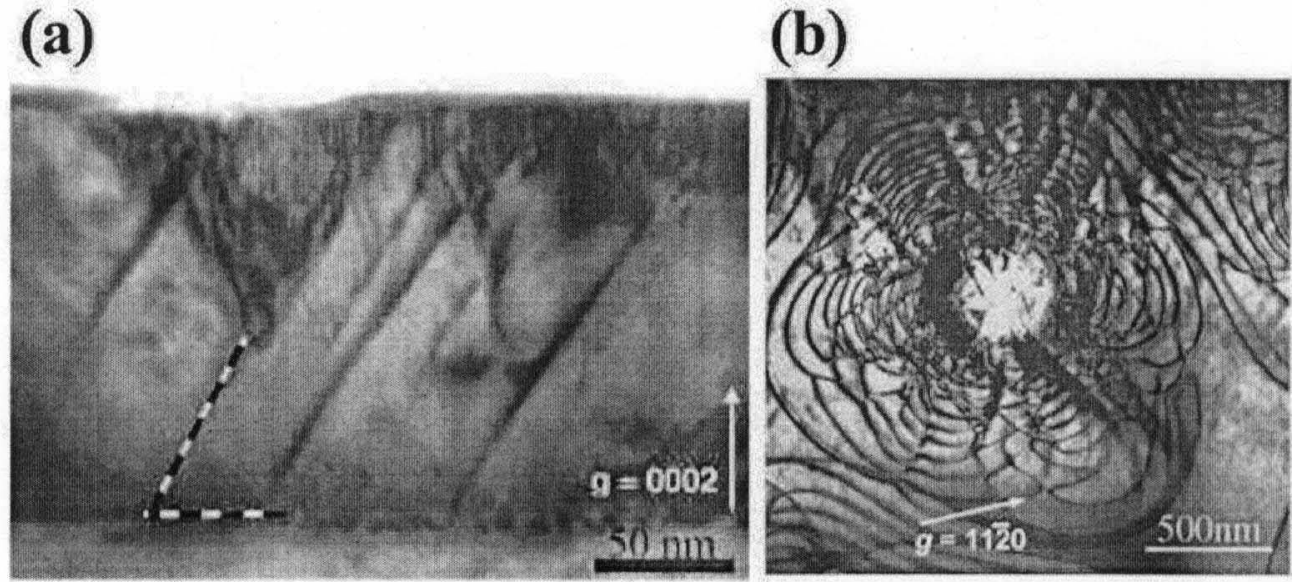

Figure 2.7 Manifestations of strain relaxation in $\mathrm{InGaN}$ films on $\mathrm{GaN}$ substrates. a)

Formation of displaced triangular sections due to slip along the planes b) formation of dislocation half loops via the introduction of misfit dislocations ${ }^{139}$.

The role of the lattice mismatched substrate on the InGaN growth is also demonstrated with studies of $\mathrm{InGaN}$ growth on a GaN layer on sapphire as well as on GaN layers grown via epitaxial lateral overgrowth (ELOG) method $^{134}$. It was observed that for a traditional film, initial InGaN growth is pseudomorphic where the threading dislocations migrate from the substrate into the film opening up into pyramidal pits as shown in figure 2.8 a, whereas for ELOG samples, in the absence of any dislocations in the substrate, a periodic array of dislocations is generated in the growing layers as shown in figure $2.8 \mathrm{~b}$, as means of strain relaxation. Although the composition distribution was not analyzed in the study, the granular appearance in figure $2.8 \mathrm{a}$ as well as the strained regions in figure $2.8 \mathrm{~b}$ could be indicative of phase segregation. 


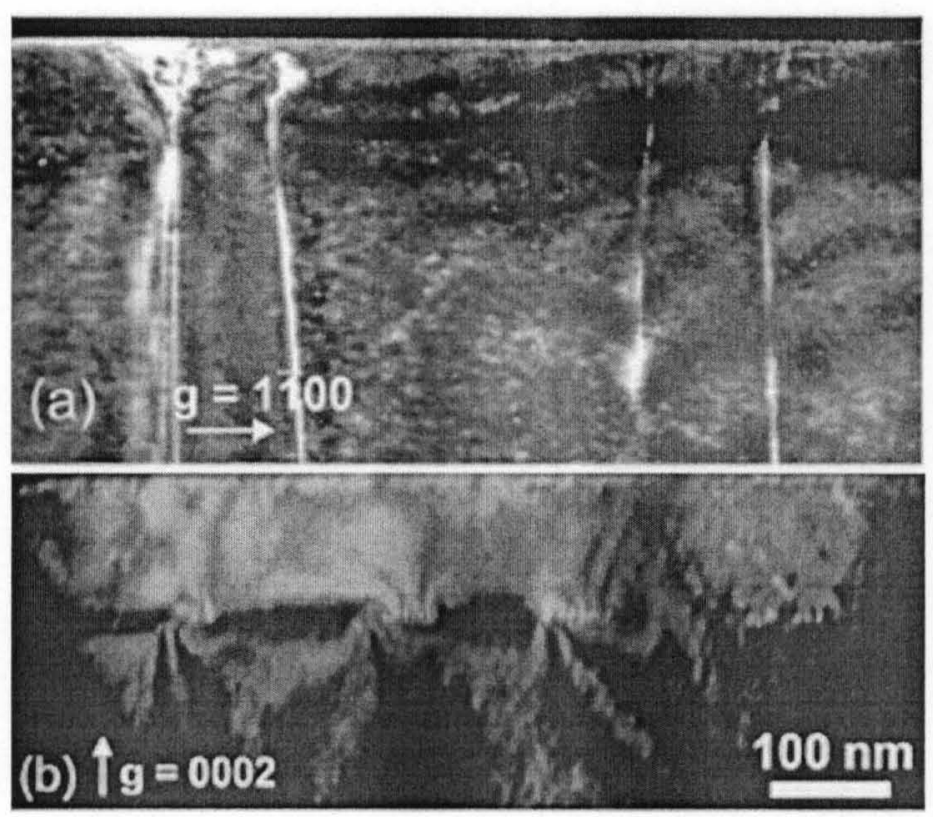

Figure 2.8 Manifestations of strain relaxation in InGaN films showing the role of dislocations in the substrates. a) Propagation of dislocations from the substrate into the growing film forming pyramidal pits for growth on regular substrate while b) Formation of periodic array of misfit dislocations for growth on ELOG grown films ${ }^{141}$.

Formation of etch pits of varying sizes was observed for the growth of $200 \mathrm{~nm}$ thick InGaN films on GaN substrates ${ }^{142}$. Three distinct growth modes were observed. Small etch pits with indium rich regions were observed to develop at the $\mathrm{InGaN} / \mathrm{GaN}$ interface which would be due to the opening up of a threading dislocation at the interface as shown in figure 2.9 a. Very large V-pits running through the InGaN as well as the GaN layers were also observed as shown in figure $2.9 \mathrm{~b}$. These could be due to the threading dislocations that open at the $\mathrm{GaN} / \mathrm{sapphire} \mathrm{interface.} \mathrm{A} \mathrm{third} \mathrm{kind} \mathrm{of} \mathrm{pit} \mathrm{formation} \mathrm{shown}$ in figure $2.9 \mathrm{c}$ was observed for growth at high temperatures and it attributed to thermal 
erosion where the dynamics of the absorption and desorption processes shift towards desorption causing the pit formation. The formation of indium rich region at the base of the pits in figure $2.9 \mathrm{a}$ is a clear indicative of phase separation.
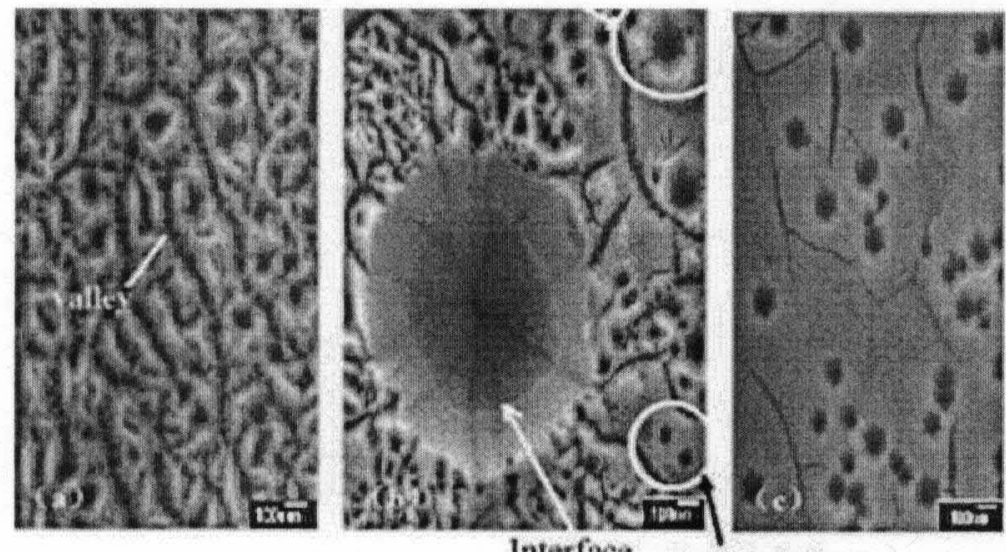

Interface In-rich defect

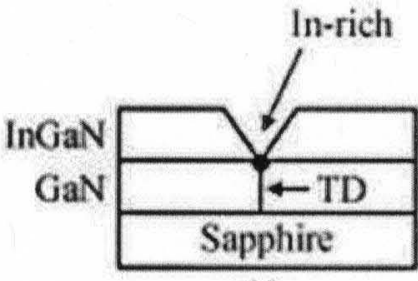

(a)

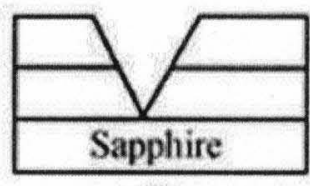

(b)

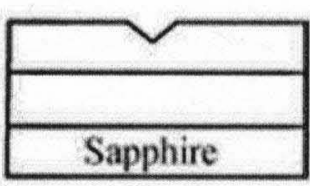

(c)

Figure 2.9 Observed morphology of InGaN growth on GaN substrates. a) Small pits formed due to the opening of dislocations at the InGaN/GaN interface. b) Large pits running all the way into the GaN layer, formed due to the opening of the dislocations at the GaN/Sapphire interface. c) pits formed due to thermal erosion ${ }^{142}$.

Various other manifestations of dislocations ${ }^{141,143,144,145,146}$ have been observed for the growth of InGaN films on different substrates and for different film parameters. Such a wide variety can be explained by the fact that each condition might make the formation 
of certain defects or dislocations energetically favorable. Such dislocations have also been shown to cause preferential drift of charge carriers ${ }^{147}$. Competing film distortion or formation of islands as a strain relaxing mechanism was observed to lead to a reduction in dislocations ${ }^{148}$.

Formation of quantum dots in InGaN layers is also a manifestation of strain relaxed growth ${ }^{149}$. Strong emission observed in InGaN/GaN multi quantum wells was shown to be due to the formation of indium rich quantum dots in the active layers due to phase segregation ${ }^{150}$. This is the only case where phase segregation is useful. In water splitting, the presence of indium rich regions can act as radiative recombination sites where the generated charge carriers will be annihilated, severely limiting conversion efficiency. The presence of indium rich phase on the surface can also cause degradation of the material in solution as high indium content alloys are unstable in solutions.

High indium content thick InGaN films (300-400 nm) on GaN have been studied to have high dislocation densities as well as planar defects ${ }^{151}$. Also, the InGaN films were observed to segregate into indium and gallium rich regions. In the same study, phase stable thin InGaN layers with high indium content $(53 \%)$ were synthesized via double heterostructure layer $(\mathrm{GaN} / \mathrm{InGaN} / \mathrm{GaN})$. The strain from $\mathrm{GaN}$ from both sides could be acting towards mitigating the phase segregation. However, such structures cannot be used for device applications as the top $\mathrm{GaN}$ layer will interfere. Other alternatives such as using buffer layers have been employed to reduce the lattice mismatch and hence phase segregation. This involves the synthesis of a thin buffer layer of GaN or $\mathrm{AlN}^{59,152}$. Stacks of buffer layers/graded $\mathrm{InGaN}^{153}$ have also been used for strain relaxed growth. However, 
the buffer layers can act as recombination centers affecting the charge transport across the interface.

Quantum wells can be grown without phase segregation while growing thick InGaN films leads to build up of strain that can induce dislocations, cause phase segregation or polycrystallinity. Kinetic controlled growth in MOCVD and MBE have been shown to improve phase stability in InGaN alloys. However, such methods have also been prone to phase segregation for thick layers.

There are numerous reports of growth of InGaN films and quantum well structures. The generic theme of these articles is to study the phase segregation or report the synthesis of phase stability. A careful classification of this leads to the observation that there have been no reports of thick "direct InGaN on a substrate" film synthesis without phase segregation. Calculations incorporating the effect of strain on the system show that strain can also depress phase segregation in the low indium content alloys ${ }^{154}$. The high indium content alloys needed for a PEC material are still prone to phase segregation. The reports of phase stable synthesis have been on quantum wells where the growth is pseudomorphic and/or the strain build up is not large enough to cause phase separation. The reports on thicker films growth are only at low indium compositions ${ }^{154}$ while phase segregation is observed at large indium compositions ${ }^{48}$. Phase segregation at the mid indium composition region is a consistent observation ${ }^{155}$.

Research has been shifted to alter the epitaxial growth where the stress on the growing layers can be reduced. In some cases, a high growth rate is employed to achieve phase stability. In this method, the critical thickness was found to increase with increasing growth rate providing a two-fold advantage in terms of growth time and 
stability. The other approach is the use of a buffer layer on the substrate usually GaN layer grown at low temperatures that acts as a strain relaxing layer. Most of the studies on heteroepitaxial growth of InGaN employ some kind of buffer layer. It is useful for growth studies but has major disadvantages. It is not desirable for device applications as it can enhance recombination due to its polycrystalline morphology with grain boundaries and reduced charge transport properties. Also, scientific studies to understand the morphology of heteroepitaxial growth cannot tolerate a layer between the substrate and the growth layer that will hinder a pure heteroepitaxial growth.

$\mathrm{ZnO}$ has been used as a close to lattice matching substrate for phase stable growth of thin InGaN layers $(\sim 80 \mathrm{~nm})$ with up to $43 \%$ indium ${ }^{156}$. Larger phase separation was observed for growth on sapphire compared to $\mathrm{ZnO}$. However, this study also used a buffer layer of $\mathrm{GaN}$ on the $\mathrm{ZnO}$ substrate. Moreover, synthesis of single crystalline $\mathrm{ZnO}$ substrates is a very expensive process. Lattice matched substrate obtained via growth of strained layers the growth of $200 \mathrm{~nm}$ thick InGaN layers on GaN and AlN layers ${ }^{157}$ on $\mathrm{Al}_{2} \mathrm{O}_{3}$ where the phase stability was attributed to strained substrates, fast growth rate and low temperature growth. It could be that the strained $\mathrm{GaN}$ and $\mathrm{AlN}$ substrates could act as close to lattice matched substrates for InGaN growth. This could be used as a way to develop lattice matched substrates for many heteroepitaxial systems. Strain has been surprisingly shown to mitigate phase segregation to some extent in low indium content films ${ }^{44}$. The strain effect increased with increase in indium content by shifting the immiscibility gap into areas of higher indium content.

Another approach to achieve high indium content InGaN layers without phase segregation is to use double heterostructure ${ }^{151}$ where $\mathrm{InGaN}$ is stacked between two GaN 
films. This is another manifestation of using strain to mitigate phase segregation. The presence of GaN however, would interfere with direct access to carriers generated in the InGaN layers.

\subsection{Heteroepitaxial growth on nanowires substrates}

Nanowires are the modern frontiers of materials technology. Apart from providing huge surface area enhancement for the same amount of material compared to planar films, nanowires also offer advantages in terms of providing a single crystalline channel for carrier transport. The interest in nanowires was only for the growth of quantum wells and growth as nanowires. However, the fact that compared to planar substrates where the adatoms diffusion is not spatially constrained nanowires constrains the adatom diffusion in two dimensions and could have interesting growth kinetics when used as substrates. Most of the work on nanowires has been focused on the growth of InGaN radial multi quantum wells for LED applications where the layers are 2-5 nm thick. They are not suitable for absorption and do not demonstrate the advantages of nanowire architectures.

Growth of a lattice mismatched shell on a nanowire with small diameters would induce a strain in the core. It has been estimated that the strain will be shared by the core and the shell which would increase the critical thickness for phase segregation to values

higher than that for growth on thin films ${ }^{50}$. Thick InGaN layers grown on $\mathrm{GaN}$ nanowires of diameters $>100 \mathrm{~nm}$ show strain dependent variation in indium content which leads to non-uniform InGaN composition ${ }^{49}$. Threading dislocations were observed in the InGaN 
films and the fact that indium content was not even in the mid composition regions $(<$ $35 \%)$ corroborates our idea that nanowires with diameters $(>50-100 \mathrm{~nm})$ act as planar substrates and do not aid in strain relaxation to reduce phase segregation. Phase separation via the formation of aluminum rich regions at the intersection of two facets was observed during the growth of thin $50 \mathrm{~nm}$ AlInP alloy films on GaAs nanowires ${ }^{158}$ and was attributed to variation in chemical potential and species diffusion.

Summarizing, there is very little work on heteroepitaxial growth on nanowire substrates and the work done has been mostly with very thin films or thick nanowires. Neither of which can be used to demonstrate the true potential of nanowires as substrates for heteroepitaxial growth. 
CHAPTER 3

EXPERIMENTAL

\subsection{Introduction}

This chapter includes details on the synthesis methods for $\mathrm{GaN}, \mathrm{GaSb}$ and $\mathrm{InSb}$ nanowires and InGaN layers on $\mathrm{GaN}$ nanowires. Bulk and nanoscale, structural and optical characterization techniques for identification of the phases as well as composition and uniformity are also discussed. The procedure employed for the measurement of photoactivity of the synthesized materials is also included.

All the binary ( $\mathrm{GaN}, \mathrm{GaSb}$ and $\mathrm{InSb})$ nanowires were synthesized in a CVD reactor using generic thermal CVD with material specific variations. The ternary InGaN layers were synthesized in a home built MOCVD reactor. All the synthesized nanowire based structures were characterized for phase, crystallinity and composition via electron microscopy, X-ray diffraction, Raman and Photoluminescence and diffuse reflectance measurements.

\subsection{Synthesis}


Synthesis in this study is divided into nanowire synthesis and synthesis on nanowire substrates. Binary materials ( $\mathrm{GaSb}$ and $\mathrm{InSb}$ ) synthesis via different controls was performed to study the growth mechanism of nanowires by controlling the experimental parameters deduced from the phase diagram. GaN nanowires with controlled growth orientation and diameters were synthesized to act as substrates for the growth of stable InGaN alloys. InGaN alloys with controlled composition were synthesized on the nanowire substrates to study the efficacy of nanowires as growth substrates.

\subsubsection{GaN nanowire synthesis}

$\mathrm{GaN}$ nanowires were synthesized in a thermal CVD reactor shown in Figure 3.1. It consists of a stainless steel chamber 6 inch in diameter and 12 inch long. The chamber has water cooled walls as well as ports for gas inlet, pressure measurement and connection to vacuum pump. The heating source is a serpentine tungsten filament $(0.035$ inch thick, from tungsten.com) that radiatively heats the silicon carbide coated graphite susceptor, placed $1 \mathrm{~cm}$ above the heater as shown in the figure 3.1 . The source and the substrate are placed directly on the susceptor and a thermocouple placed on the susceptor measures the temperature. Since the heating is primarily via radiation, the filament goes to temperatures in excess of $2300 \mathrm{C}$ for the susceptor (and the substrate) to reach the temperatures of $800-1000 \mathrm{C}$ for $\mathrm{GaN}$ synthesis. This has an added advantage as the filament cracks the ammonia gas into nitrogen and hydrogen species creating a pseudoplasma atmosphere that aids the reactions. 


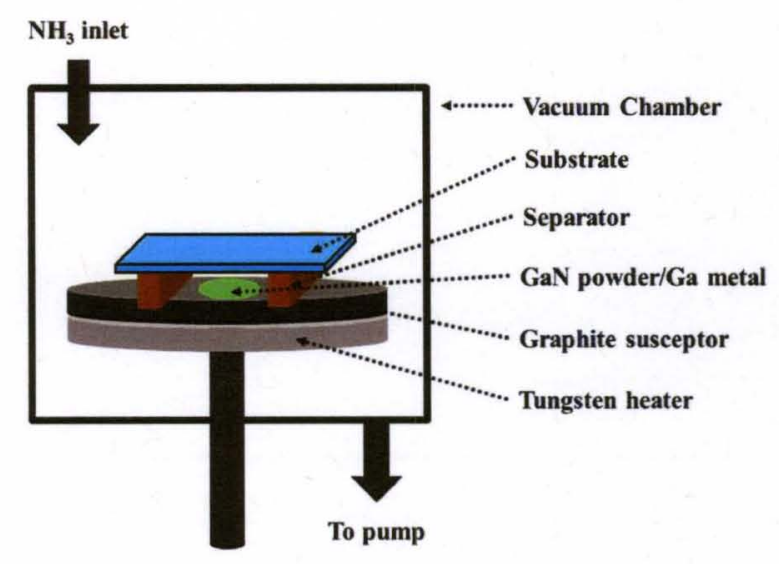

Figure 3.1 Schematic of the reactor illustrating the heater, substrate and source material position. In the case of $\mathrm{GaN}$ powder synthesis, only gallium metal was placed on the susceptor while for $\mathrm{c}-\mathrm{GaN}$ nanowires, $\mathrm{GaN}$ powder along with the substrate and for aGaN nanowires gallium metal and the substrate were used.

\section{“c" plane GaN nanowires}

$\mathrm{GaN}$ powder was used as the source material for the synthesis of c-GaN nanowires. Earlier, commercial GaN powder was used for the synthesis of $\mathrm{GaN}$ nanowires. However, it was observed that powder synthesized in the lab works better. 1-2 $\mathrm{mm}$ diameter gallium metal droplets are placed on the susceptor and heated to $600 \mathrm{C}$ at $300 \mathrm{mTorr}$ pressure under $200 \mathrm{sccm} \mathrm{NH}$ flow. Partial nitridation is achieved in $30 \mathrm{~min}$ where the gallium metal spreads onto the susceptor and dark grey colored GaN powder is obtained.

The setup used in c-GaN nanowire synthesis is illustrated in Fig 3.1. The required substrate (stainless steel, copper, quartz, silicon and $\mathrm{GaN}$ coated sapphire) is placed with the growth surface facing and at a distance of $2-3 \mathrm{~mm}$ from the GaN powder using 
graphite piece supports. The chamber is pressurized to 30 Torr using $200 \mathrm{sccm} \mathrm{NH}$ and heated to $950 \mathrm{C}$.

\section{"a" plane GaN nanowires}

Gallium metal was used as the gallium source along with $\mathrm{NH}_{3}$ for the synthesis of a-GaN nanowires. The experimental setup used is very similar to that used for the synthesis of c-GaN nanowires (Fig 3.1). Gallium metal is placed on the susceptor and the substrate is placed $2-3 \mathrm{~mm}$ above using graphite pieces as separators. The susceptor is heated to $725 \mathrm{C}$ under $200 \mathrm{sccm} \mathrm{NH}$ flow at $250 \mathrm{mTorr}$ pressure and maintained for 30 $\min$.

\subsubsection{GaSb nanowire synthesis}

GaSb nanowires were synthesized using two different methods 1) Antimony tip led and 2) Gallium tip led growth, to demonstrate the different types and control over the nanowire growth modes.

\section{Antimony tip led GaSb nanowires}

The reactor setup is a little different than the one used for GaN nanowire synthesis. The chamber is the same but instead of the tungsten filament a BN (boron nitride) heater was used. Also, the source materials (Gallium and Antimony) were placed in a $\mathrm{BN}$ (boron nitride) crucible resting on the $\mathrm{BN}$ heater as shown in the schematic in 
Fig 3.2. The substrate is placed on the crucible with the growth surface placed face down. The system is heated to $735 \mathrm{C}$ under $35 \mathrm{sccm} \mathrm{H} 2$ flow at $150 \mathrm{~m}$ Torr pressure.

\section{Gallium tip led GaSb nanowires}

A setup similar to that in Fig 3.2 is employed for synthesis of gallium tip led $\mathrm{GaSb}$ nanowires. Instead of using gallium droplets as gallium source, a gallium coated quartz piece is used as a substrate and is placed on a $\mathrm{BN}$ crucible with antimony powder in it. The system is heated to $700 \mathrm{C}$ under $50 \mathrm{sccm} \mathrm{H} 2$ at $200 \mathrm{mTorr}$ pressure. Gallium is smeared onto a quartz piece using another quartz piece until a film with mirror finish is obtained.

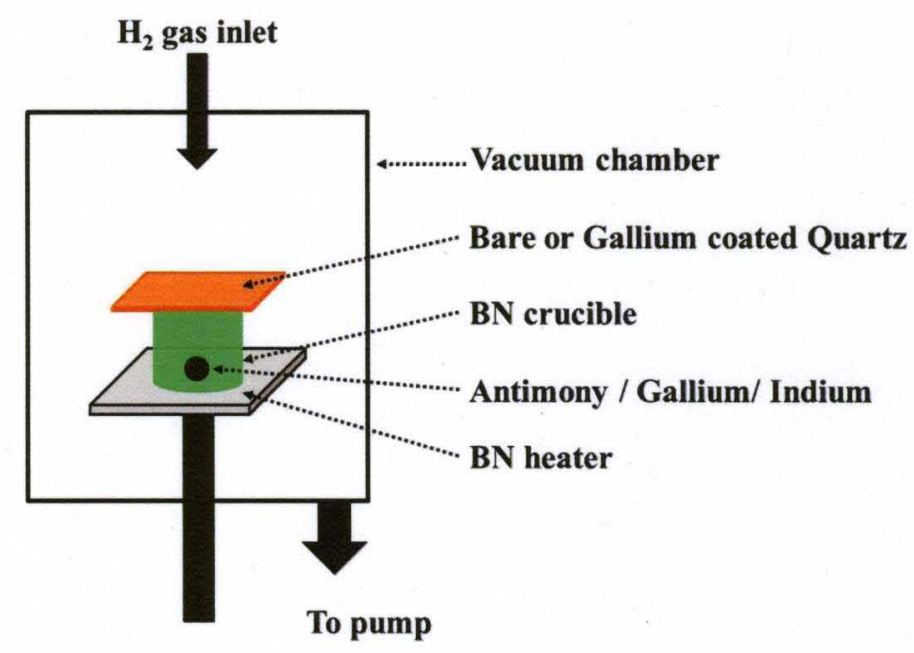

Figure 3.2 Schematic of the reactor illustrating the heater, source and substrate set up for III-Sb nanowire synthesis. 


\subsubsection{InSb nanowire synthesis}

InSb nanowire synthesis experiments were designed to be in the excess antimony conditions, reasons for which will be discussed later.

\section{Antimony tip led InSb nanowires}

InSb nanowires were synthesized using a similar setup shown in Fig 3.2. Indium metal and antimony powder, the source materials were placed in a BN crucible with a quartz piece on top. The crucible was placed on the $\mathrm{BN}$ heater. The system was heated to $535 \mathrm{C}$ under $50 \mathrm{sccm} \mathrm{H}_{2}$ flow at $150 \mathrm{mTorr}$ pressure. Grey deposition was observed on the quartz piece.

\subsubsection{Synthesis of InGaN alloys on GaN nanowire substrates}

InGaN layers were grown on $\mathrm{GaN}$ nanowires of controlled orientation to study the effect of nanowires as substrates as stress relaxing substrates.

Synthesis was performed via MOCVD process in a home built reactor. Details on the reaction design, assembly and operation for precursor flow control are provided in chapter 5. The reactor consists of a multi precursor delivery system where a carrier gas is used to transport the metal organic precursors into the reaction chamber where they react and deposit onto the desired substrate. TMGa (Trimethyl Gallium), TMIn (Trimethyl Indium) and $\mathrm{NH}_{3}$ were used as the gallium, indium and nitrogen sources respectively for InGaN synthesis. Composition control is obtained by controlling (the parameters that 
control the incorporation rates of gallium and indium species) temperature, pressure and material flux). GaN nanowires grown on stainless steel substrate are used as substrates for the growth of InGaN layers. InGaN growth conditions were maintained within the temperature ranges employed for $\mathrm{GaN}(900 \mathrm{C})$ and $\mathrm{InN}(550 \mathrm{C})$ synthesis. The system was maintained at high pressures of about 100 Torr to reduce indium losses via evaporation.

\subsection{Material Characterization}

Structural characterization tools used for the studies include scanning electron microscope (SEM), transmission electron microscope (TEM), X-ray diffraction (XRD) and Raman spectroscopy. Optical properties of the materials as well as compositions in the case of ternary InGaN were measured using diffuse reflectance (DR) and photoluminescence (PL) measurements. Elemental composition and uniformity was measured using energy dispersive spectroscopy (EDS) in SEM and TEM while convergent beam electron diffraction (CBED) and selected area electron diffraction (SAED) were used for localized composition and crystallinity studies. Energy filtered TEM maps were also performed on the InGaN growth to study the localized material distribution in the layers. These techniques will be introduced briefly in the following sections. 


\subsubsection{Diffuse Reflectance (DR)}

Diffuse reflectance is a variation of absorbance measurement. This is particularly useful for the case of measurement of materials on opaque substrates where absorbance and transmission measurements are simply not possible. The measured reflectance from the sample is converted to usable data format to measure the band gap of the material.

As shown in the schematic in fig 3.3, the sample is not placed normal but at a small tilt from the incident beam to eliminate all specular reflection. The sample, beam and detector are housed at the periphery of an integrating sphere coated with a standard reflecting material. Light falls on the material at an angle different that normal eliminating specular reflection. The diffuse reflectance rays that are generated at multitude of angles are then focused onto the detector by the reflective coating.

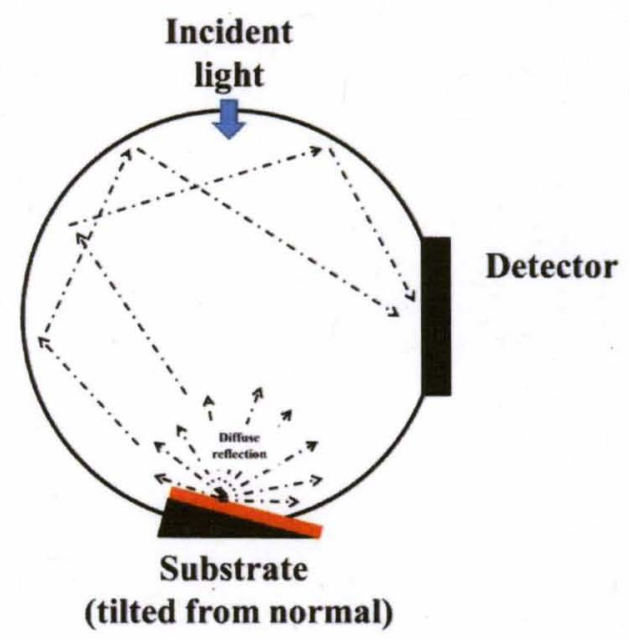

Figure 3.3 Schematic illustrating the instrument and principle of Diffuse reflectance measurement. 
The collected reflectance data $(R)$ is converted using the following relation

$$
C=\left[\ln \frac{\left(R-R_{\min }\right)}{\left(R_{\max }-R_{\min }\right)}\right]^{2}
$$

A plot of $\mathrm{C}$ vs energy is used to determine the band gap of the material ${ }^{159}$. Extrapolation of the vertical segment of the plot into the energy axis is the band gap and the plot being vertical indicates the band gap being direct.

\subsubsection{Selected area diffraction (SAED)}

SAED is a diffraction technique that is performed in a transmission electron microscope. The incident parallel beam of high energy electrons (has wavelength in sub angstrom lengths) shoots through the $\sim 100 \mathrm{~nm}$ thick specimen. The specimen here acts as a diffraction grating for the electron wave generating a spot pattern as shown in the schematic in figure 3.4. The generated pattern can be used to identify various properties of a crystal. The technique is termed as SELECTED area electron diffraction as very small parts of the sample can be selectively and independently studied under the electron beam. This is achieved using an aperture which is a metal sheet with holes of predetermined sizes that only send a small part of the beam making localized measurements possible. 


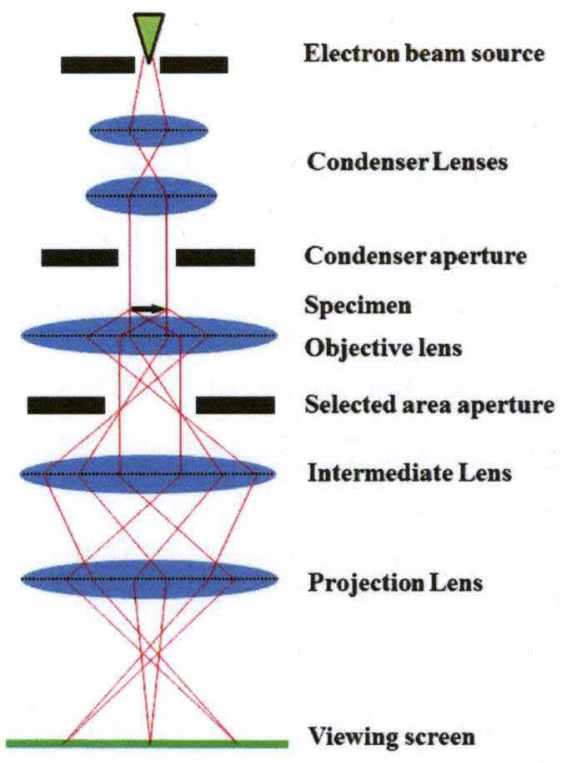

Figure 3.4 Schematic of the spot pattern formation in SAED.

Here, measuring a single crystal is the ideal case as polycrystalline material has crystals with random orientation that can give rise to a bunch of spot patterns which would be impossible to deconvolute.

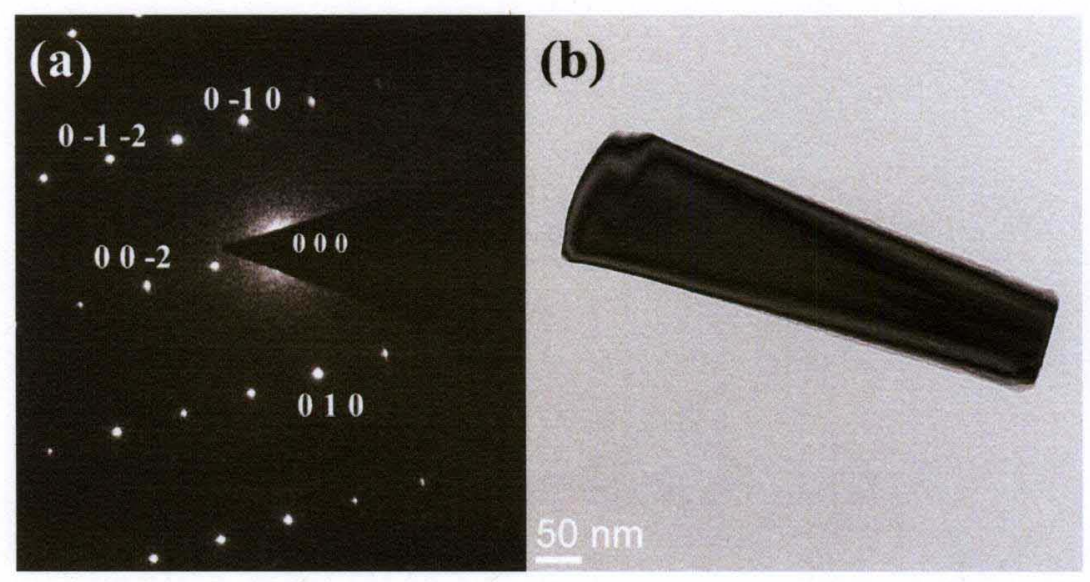

Figure 3.5 a) SAED spot pattern from the GaN nanowire in b) tilted to [100] zone axis 
A sample diffraction pattern is shown in fig 3.5 a generated from the $\mathrm{GaN}$ nanowires shown in figure $3.5 \mathrm{~b}$. The spots are the projections of the reciprocal lattice and lattice reflections show up as the diffraction spots. SAED can be used to measure lattice spacing and identify crystal structures by tilting the crystal to low index zone axes. SAED is also useful in the analysis of interfaces, twinning and defects. In this study, we use SAED to measure the lattice constant and correlate it to the composition of the InGaN alloy.

\subsubsection{Convergent beam electron diffraction (CBED)}

The main different between SAED and CBED is the manner of the beam hitting the sample. It is a parallel beam on the specimen limited by the aperture size in the case of SAED while the beam is focused on the sample in the case of CBED. Hence, CBED can be used for better resolution measurements. The difference in the two measurements is that SAED results in a spot pattern while the CBED results in a pattern of discs. The image formation is shown in the schematic in figure 3.5. 


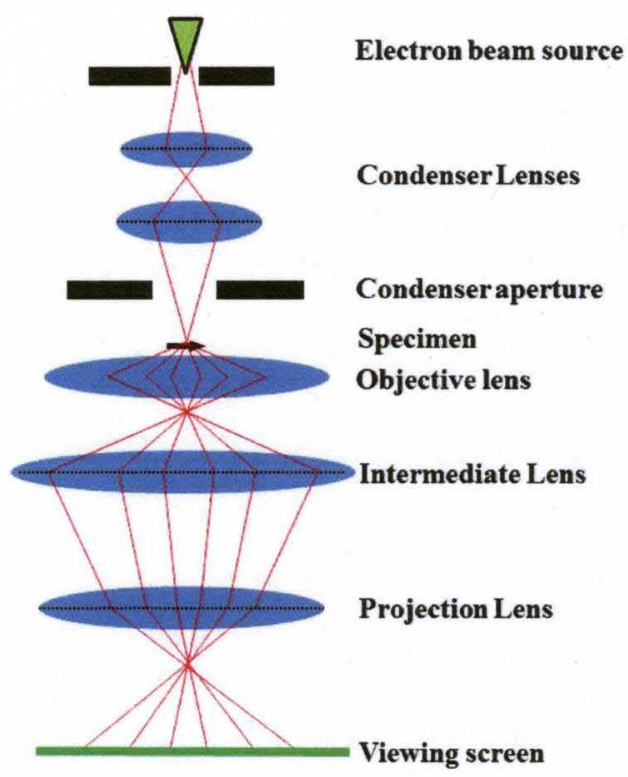

Figure 3.6 Schematic of the formation of discs in CBED

A representative CBED pattern from a GaN nanowire is shown in figure 3.7a. The bright spot on the nanowire in figure $3.7 \mathrm{~b}$ is the entire spot that was used to generate the disc pattern. The disc patterns can be used to calculate the lattice spacing to start with. CBED patterns can be used to deduce the polarity,

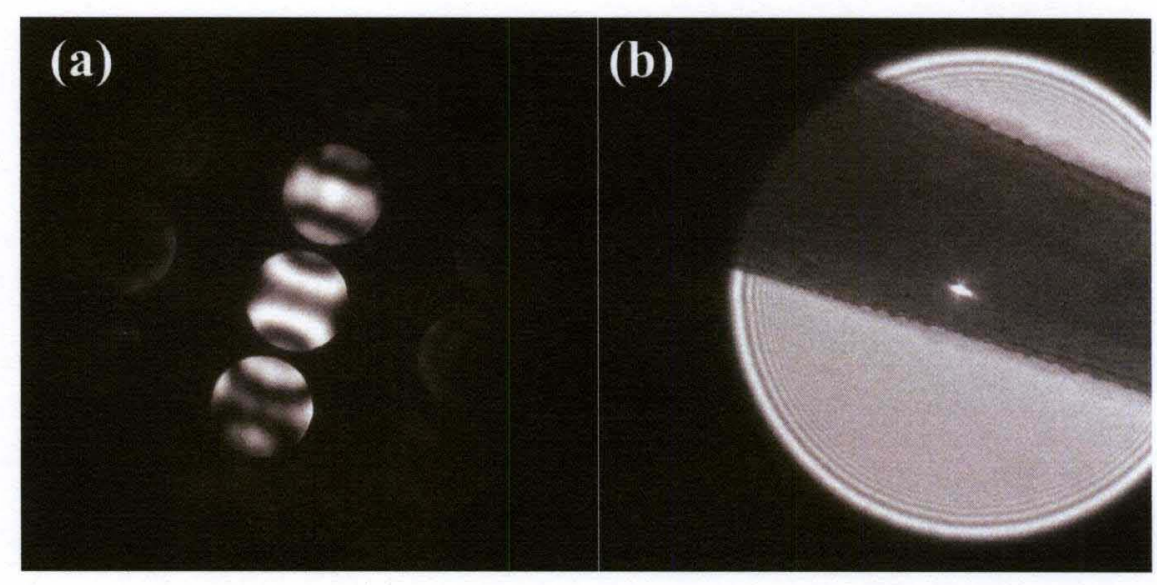

Figure 3.7a is a sample CBED pattern from the bright spot on a GaN nanowire in b). 
The advantages of a CBED pattern are that it is much more localized, the orientation is more accurate, and hence the data is more reliable. This can also be used to measure specimen thickness and strain in the material.

\subsection{Photoactivity measurements}

Photoactivity measurements involve demonstrating the generation of water splitting properties of the material. Towards this, photo-voltage and photo-current measurements were conducted.

\subsubsection{Electrode fabrication}

The stainless steel substrates with the $\mathrm{InGaN} / \mathrm{GaN}$ nanowires were attached to a copper wire and all the exposed metal surface was coated with epoxy to prevent any shorting as shown in figure 3.8

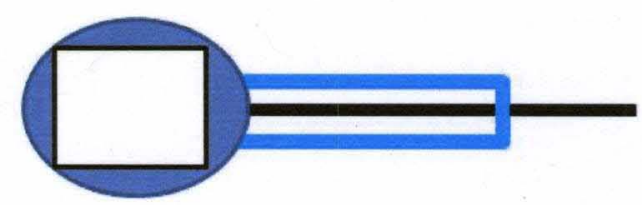

Figure 3.8 Schematic of the appearance of the electrode after the glass sheath is attached and all the epoxies are applied and cured. 


\subsubsection{Illuminated Open Circuit Potential measurement}

Apart from being an indicator of the material being photoactive, photo-voltage (illuminated OCP) measurements can be used to determine the material being n-type or p-type. This measurement can be performed in a two or three electrode setup. The two electrode setup has the counter and reference electrodes shorted. Figure 3.9 shows the schematic of the experimental setup used for photoactivity measurements.

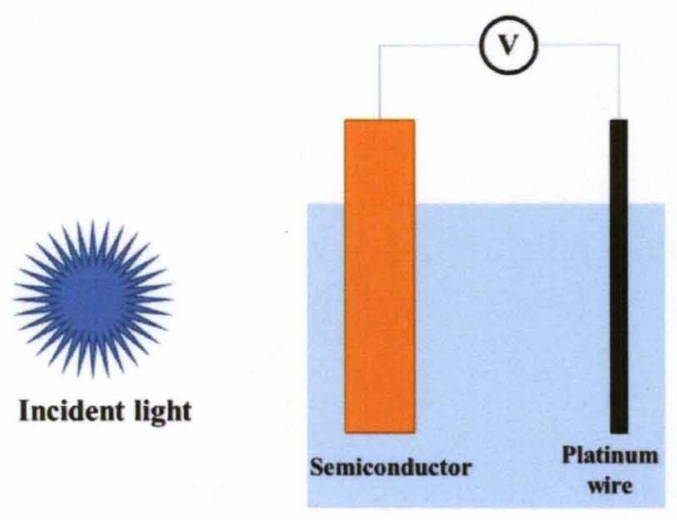

Figure 3.9 Schematic of the illuminated OCP measurement to check the photoactivity of the sample.

The change in potential is measured with time in uniform cycles of dark and illumination. A consistent switching to background values in both cases over long periods of time indicates a stable electrode. Power dependence of OCP can be studied and used to determine the flat-band potential of the electrode. 


\subsubsection{Photocurrent measurement}

Photocurrent measurements show whether the semiconductor actually performs as a photoactive material. In this study, a three electrode setup shown in figure 3.10 is preferred to have the voltages measured against a standard for making comparisons and measurements reliable.

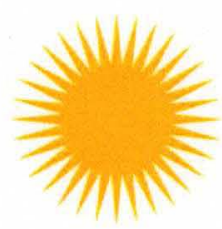

Simulated AM1.5 light

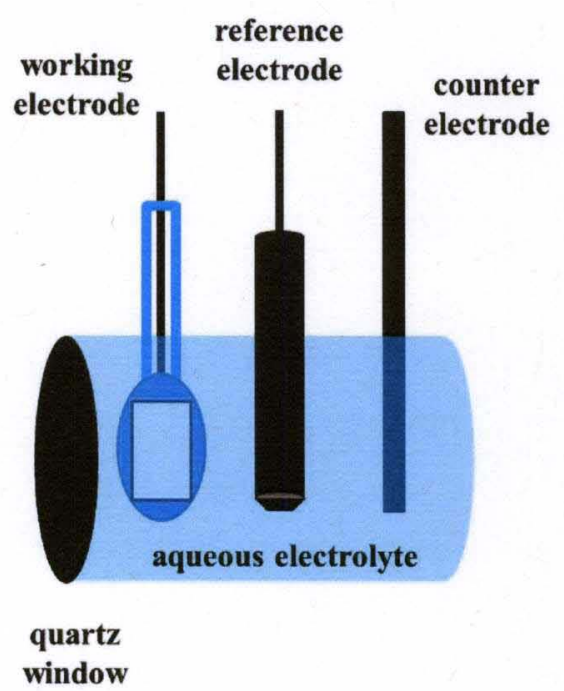

Figure 3.10 Schematic of the three electrode set up used for photocurrent measurements. InGaN/GaN on stainless steel is the working electrode and a platinum wire is the counter electrode with a $\mathrm{Ag} / \mathrm{AgCl}$ reference electrode.

Current is measured in dark and under illumination while potential is scanned from $-0.05 \mathrm{~V}$ vs OCP to $0.5 \mathrm{~V}$ to measure the change in current with illumination. In another sequence of measurements, light is chopped manually at regular intervals where one curve will show the current in dark as well as under illumination. 


\section{CHAPTER 4}

\section{MOCVD REACTOR DESIGN and MODELING}

\subsection{Introduction}

Composition controlled synthesis of ternary semiconductors need precise control over the individual constituent precursor supply. Thermal CVD systems can be modified to a dual heating zone type to synthesize ternary materials. However, the precision and scalability of the process is severely limited. A refined approach to this problem is to deploy vapor phase precursors where the flux can be independently and precisely controlled enabling composition controlled synthesis of ternary and higher order materials. MOCVD (metal organic chemical vapor deposition) and MBE (molecular beam epitaxy) are the two methods of choice. However, MBE requires very low

pressures and the growth rates are also low. MOCVD on the other hand, can be conducted at high pressures and the growth rates are much faster than in MBE. MOCVD was the preferred method for the growth of InGaN in our case.

A new reactor and precursor delivery system were designed and assembled to have a dedicated system for the synthesis of InGaN via MOCVD. This chapter includes 
the details on the design, assembly, modeling of the growth process, correlation with the experimental data and its implications on the InGaN growth.

The process of synthesis of any material via MOCVD consists of the following steps:

1. Controlled supply of precursor via a carrier gas

2. Delivery into the chamber via s showerhead

3. Control over the reactor conditions (substrate temperature, pressure) leading to the formation of the desired material.

The design parameters and criteria employed in the reactor design process in each step will be discussed. Figure 4.1 is a schematic of the MOCVD reactor system illustrating the various constituents of the system.

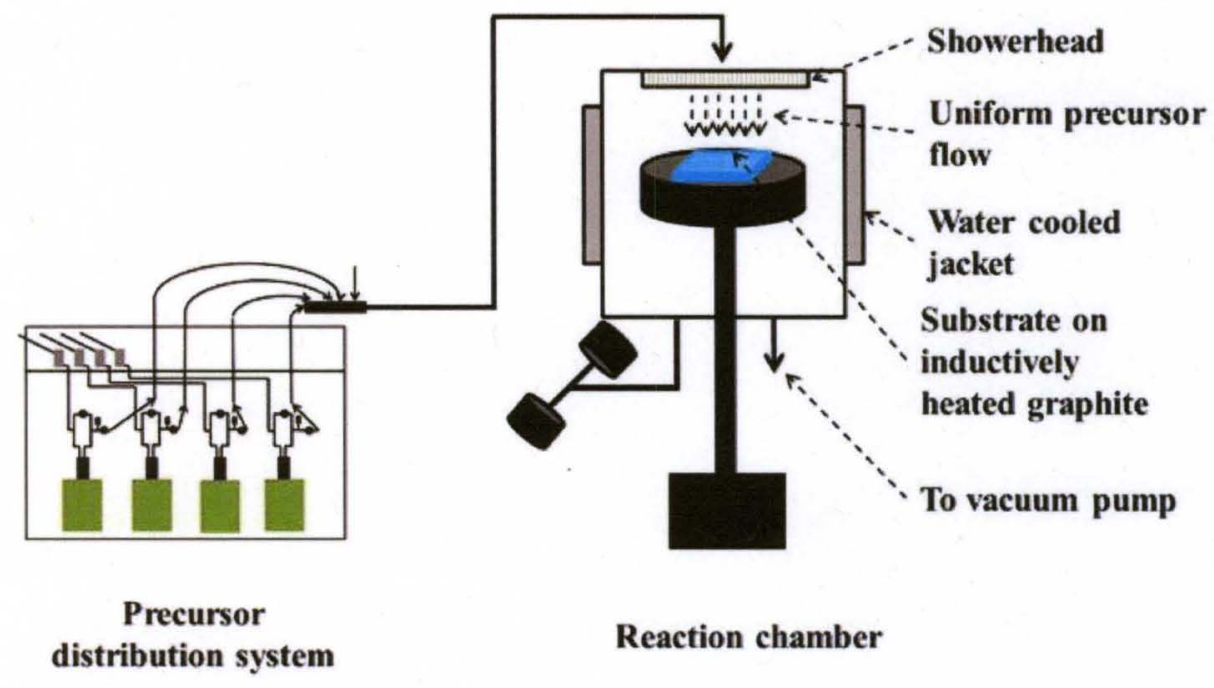

Figure 4.1 Schematic of the MOCVD reactor system. The precursor delivery system is equipped with independent controls for the supply of four precursors. The reaction 
chamber is a water cooled stainless steel vessel. The gases enter from the top via a showerhead for uniform precursor distribution onto a substrate heated using an induction heater.

\subsection{Design and assembly}

This section includes details on the design, assembly, experimental procedure and its influence on the design.

A typical experimental procedure for any MOCVD reaction is as follows:

1. The precursors have to be set to the calculated vapor pressures. It will be discussed later in the section, but each precursor has a different vapor pressure behavior which is controlled by stabilizing the bubbler in a constant controlled temperature bath. So, the precursors to be used are maintained at the desired temperatures.

2. After the sample is prepared and placed on the chamber at the right position, the chamber is pumped down to remove any moisture and oxygen. A two time cycle of pumping and purging with inert gas such as nitrogen is performed to make sure the chamber is free of oxidative species. Also, at this time, the inlet and the pump valves are closed to isolate the system and a leak check is done to make sure there are no leaks in the system. This step is also done by isolating the precursor bubbler. 
3. The reactor is pressurized to the desired value with ammonia gas and the susceptor is heated to the set temperature. Care should be taken so that the ammonia does not enter the bubbler. The system is allowed to stabilize for 15 $\min$.

4. Now, the bubbler is pressurized to the desired value using the carrier gas.

5. A known flow rate of the carrier gas is now sent through the bubbler. At a constant maintained bubbler pressure, the carrier gas now picks up the precursor vapors into the reactor.

6. The gases are sent into the reactor for a set time for the synthesis process.

7. The carrier gas flow is shut down, followed by the ammonia flow. Once the system reaches the base pressure, the heaters are turned off.

8. The reactor is vented to atmospheric pressure and sample is taken out for analysis.

Now it is this process which along with the requirements of the material synthesis that is used to guide the design process.

\subsubsection{Precursor delivery system}

The system was built with the ability to handle four precursors simultaneously for the synthesis and in situ doping of InGaN and GaSbN. Figure 4.2 is the schematic of the precursor delivery system. As shown in the figure 4.2, each delivery system is independent and all can be run simultaneously. Carrier gas is delivered independently to the inlet port of all four precursor cylinders using mass flow controllers. A bypass line is 
also configured over the bubbler for purging the lines. A control valve and a pressure gauge are attached downstream to the bubbler outlet as shown in the schematic in figure 4.3a. The carrier gas and the precursor vapors are carried into reaction chamber inlet.

Trimethyl gallium, Trimethyl Indium, Trimethyl Antimony and Bis-

cyclopentadienyl magnesium are the metalorganic precursors installed in the system.

Each material has a temperature dependent vapor pressure that can be controlled to achieve the desired flux. The vapor pressure equations for each material are shown in equations 4.1 to 4.4 .

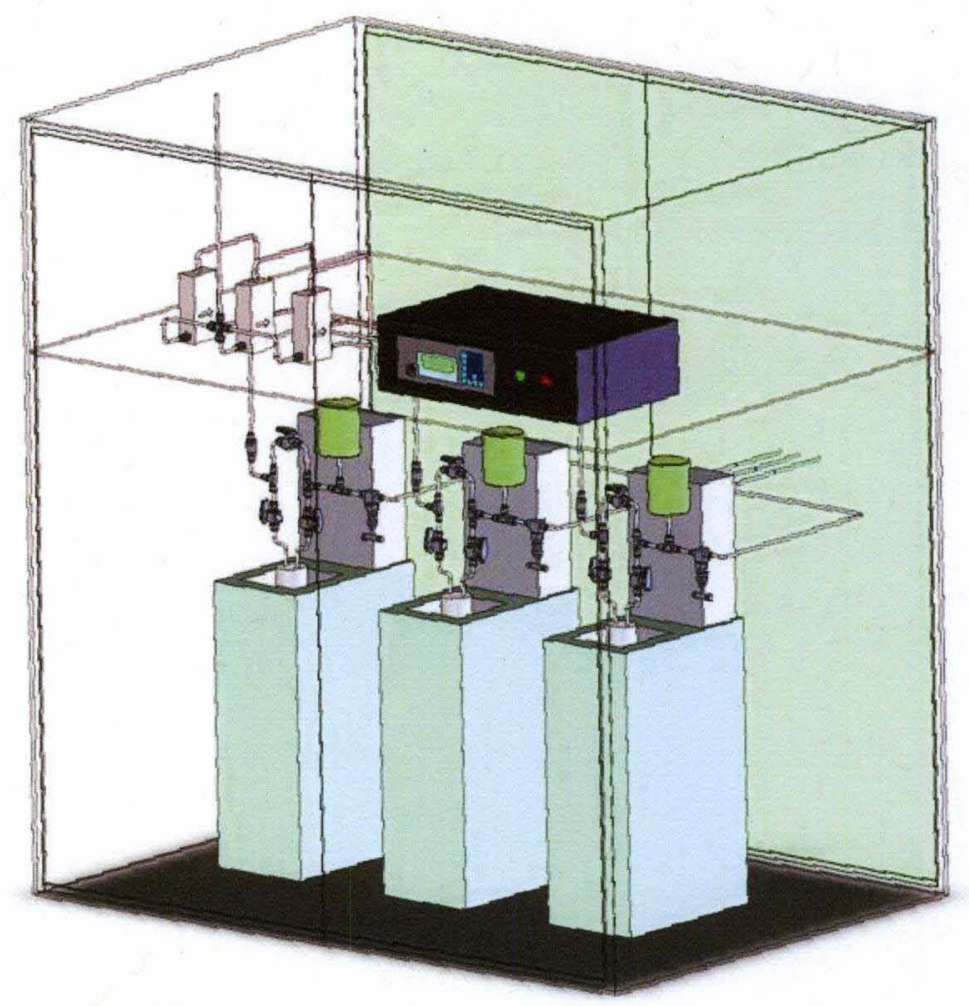

Figure 4.2 Schematic of the precursor delivery system. 
TMGa $\quad \log _{10} \mathrm{P}_{(\mathrm{mm} \mathrm{Hg})}=8.07-1703 / \mathrm{T}(\mathrm{K}) \quad 4.1$

TMIn $\quad \log _{10} \mathrm{P}_{(\mathrm{mm} \mathrm{Hg})}=10.52-3014 / \mathrm{T}(\mathrm{K}) \quad 4.2$

$\mathrm{TMSb} \quad \log _{10} \mathrm{P}_{(\mathrm{mm} \mathrm{Hg})}=7.73-1709 / \mathrm{T}(\mathrm{K})$

$\mathrm{Cp}_{2} \mathrm{Mg} \quad \log _{10} \mathrm{P}(\mathrm{mm} \mathrm{Hg})=10.00-3372 / \mathrm{T}(\mathrm{K})$

The generated vapors have to be sent to the reactor in a controlled manner with a known flux. Using direct precursor vapor delivery involves specialized and expensive mass flow controllers and back flow valves as the reactor would be at a higher pressure than the precursors. We employed a simple carrier gas based method to send the precursor into the chamber where the flux can be estimated. The precursors (solid or liquid) are contained in a bubbler to which valves and pressure measurement instruments are connected as shown in figure 4.3. The carrier gas is sent at a controlled flow rate through a mass flow controlled (independent for each precursor) to the inlet of the bubbler. A unidirectional valve is attached upstream to the bubbler. This along with the control valve downstream to the bubbler outlet is used to pressurize the bubbler with the carrier gas to attain the desired pressure.

A total bubbler pressure $\mathrm{P}$ includes contribution $\mathrm{P}_{\text {vap }}$ from the precursor that can be calculated from the equations 4.1 to 4.4 and the rest is the carrier gas. Hence, the mole fraction of the precursor in the bubbler then becomes

$$
\mathrm{X}_{\mathrm{vap}}=\left(\mathrm{P}_{\mathrm{vap}} / \mathrm{P}\right)
$$

Once the bubbler is pressurized to the set value wherein the This can be converted to molar flow rate knowing the flow rate of the carrier gas $(\mathrm{M} \mathrm{sccm})$ using the equation 4.6 ( 1 mole of any gas at standard conditions occupies $22400 \mathrm{cc}$ ) 


$$
\mathrm{M}_{\text {vap }}(\mathrm{mol} / \mathrm{min})=\mathrm{X}_{\text {vap }} * \mathrm{M} / 22400
$$

This point onwards, the precursors transported by the carrier gas can be used to send to any reactor via configurable connectors at the end of the stainless steel line. Specific to TMIn, the stainless steel lines have to be kept heated at $50 \mathrm{C}$ always as the indium can condense on the inner walls and eat away the stainless steel line over time. Heating the lines desorbs the indium leaving the tube intact.
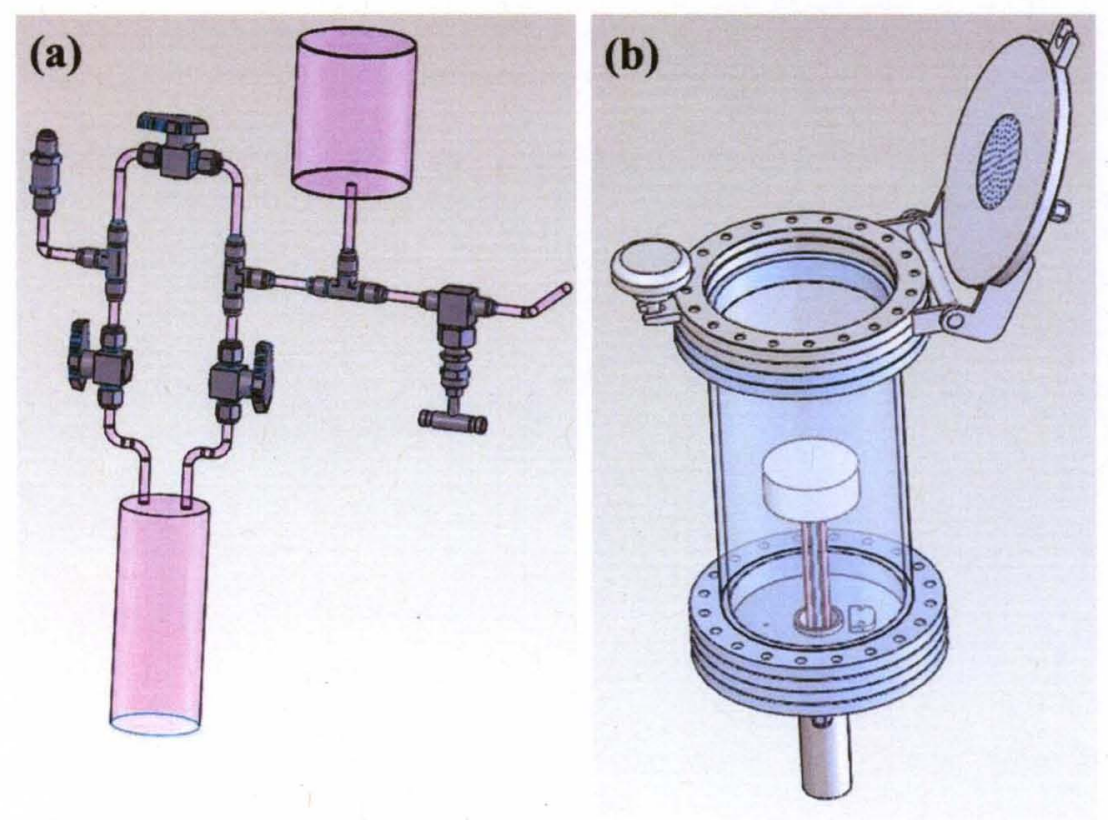

Figure 4.3 is the schematic of the bubbler and the gas pressure and flow control setup used to control the precursor flux.

Since all the metalorganic precursors are pyrophoric in nature, safety is of paramount importance. The bubblers valves should be always closed when not in use and the lines preferably should be in vacuum to maintain a clean un-oxidized inner tube 
surface as any residual precursor can oxidize. Also, the entire system is enclosed and constantly maintained under air suction into an exhaust system to contain leaks.

\subsubsection{Reaction chamber}

Figure $4.3 \mathrm{~b}$ is the schematic of the reaction chamber fabricated for the MOCVD synthesis. It consists of a top plate, the chamber enclosure, the bottom plate and the heater assembly. The top plate is a quick access door (from LDS vacuum) that was modified to include water cooling and a perforated stainless steel plate to act as the showerhead for uniform precursor distribution. The chamber is a stainless steel cylinder 8 inch in diameter and 12 inch length. The walls of the chamber were modified to add a 1 inch deep jacket for water cooling. The end connections of the chamber are 8 inch conflat flanges that match with the top and bottom plate connections. The bottom plate is a stainless steel 8 inch conflat blank plate that was modified to include access for the induction heater, pressure measurement and pumping lines. The bottom plate also has water cooling. Substrate heating is achieved using an induction heater. The induction coil also acts as a support for the graphite susceptor. The entire heater assembly can be moved up and down to change the separation between the showerhead and the substrate.

Figures $4.4 \mathrm{a}-\mathrm{b}$ are the schematics of the top plate of the reaction chamber. The plate has two opposite concentric isolated grooves. The central grove ( 2 inch diameter) opens up inside as shown in figure 4.4 a. At the end of the inner opening, a stainless steel disc with patterned $3 / 4$ inch holes is attached. This disc acts as the showerhead to break down the single stream of gas and provides a better distribution of the precursors on the 
substrate. The gas inlet connector is welded on the top plate as shown in figure $4.5 \mathrm{a}$. A 1 inch wide groove is made on the top plate for water cooling. This groove opens on the top to create a holding area for water. The opening at the top is covered with a ring that has attachments for water inlet and outlet. The two groves form two isolated chambers with a thin separation. The water on the outer ring cools the plate as well as the showerhead. The bottom plate as shown in figure $4.4 \mathrm{c}$ is modified from a solid $8 \mathrm{inch}$ stainless steel conflat blank. The plate has a grove for holding water as shown in figure $4.4 \mathrm{~d}$. There is a central 1 inch through hole for the induction heater. The pressure measurement and pumping connections are also shown in figure $4.4 \mathrm{c}$.

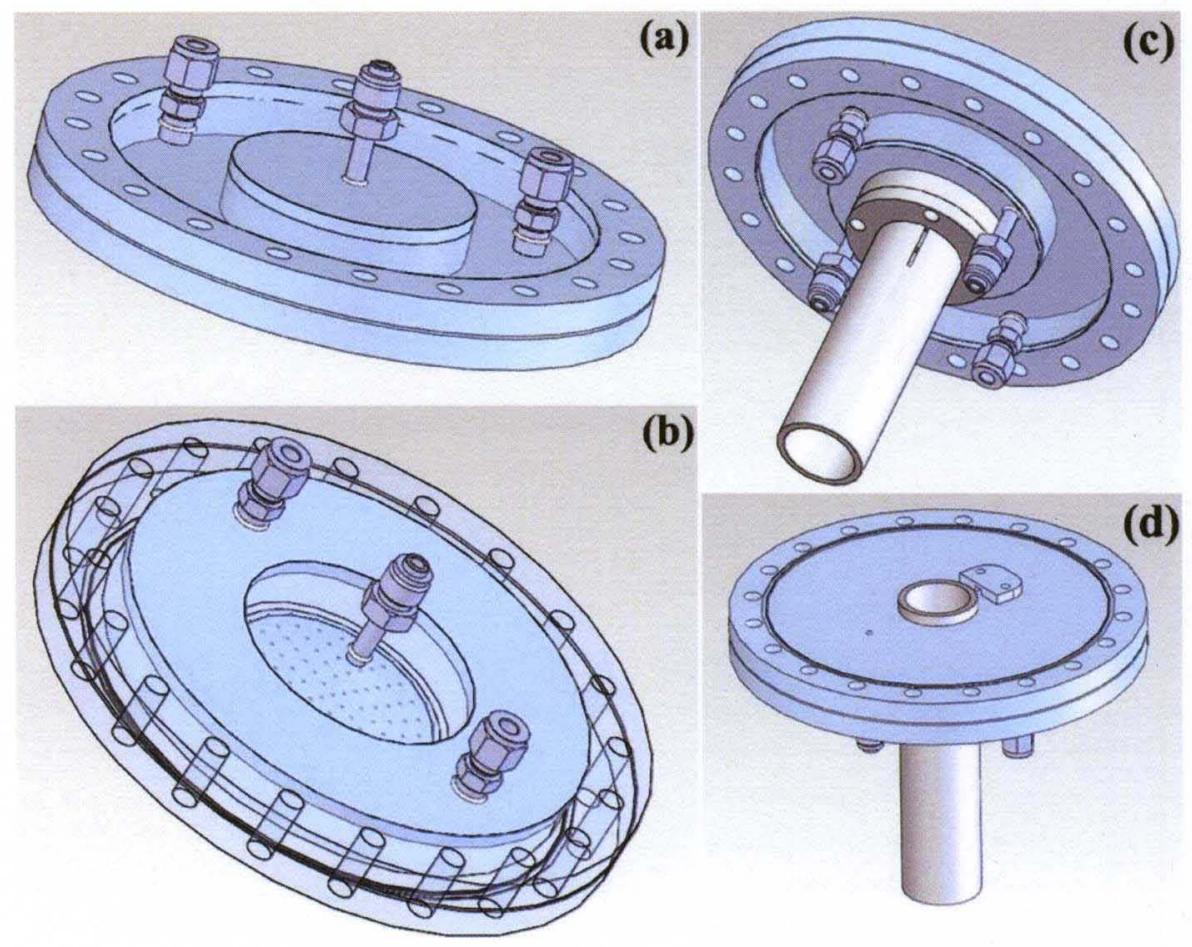

Figure 4.4 a)-b) Schematics of the top plate of the reaction chamber. Two isolated concentric groves are made in the plate. The plate has two isolated chambers for water cooling (connections at the top) and showerhead via a perforated stainless steel plate 
(opens at the bottom). c) and d) are the schematics of the bottom plate showing a similar water cooling chamber as well as the teflon sleeve for a vacuum tight sealing of the copper tube of the induction heater. The separation between the showerhead (fixed) and the substrate is changed by moving the heater assembly.

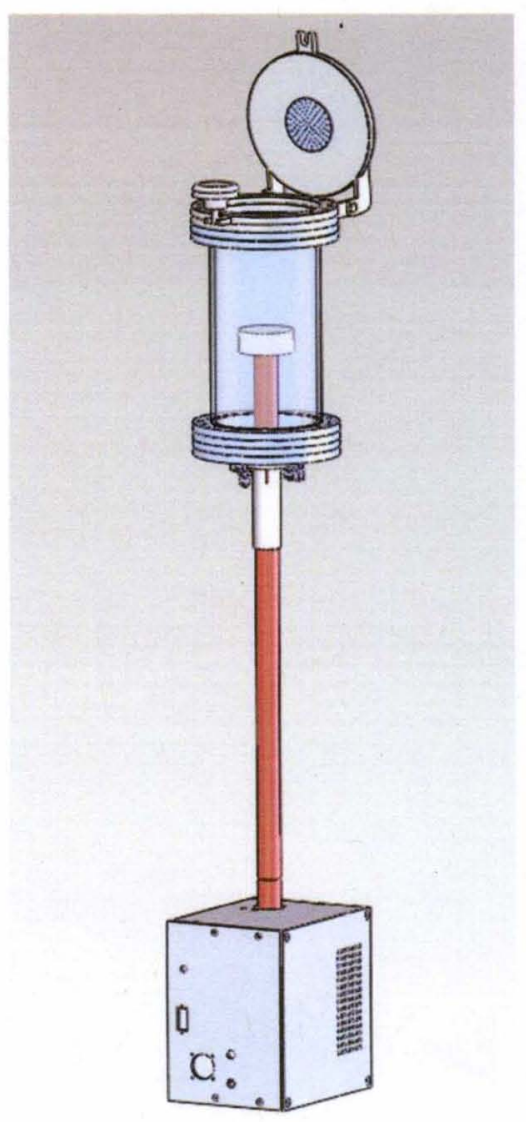

Figure 4.5 Schematic of the reaction chamber with the heater assembly showing the chamber, the showerhead, the substrate placement, the Teflon sleeve holding the copper tubing containing the copper coils for induction heating using the circuit box at the bottom. 
The gas manifold is the connection pathway for the precursor delivery system and the reaction chamber. Since there are four precursors and an ammonia line, a manifold is necessary. It has been observed that pre mixing the precursor and gases produces stray reactions that can produce unwanted nanoparticle formation in the gas phase interfering with the quality of film growth.

\subsection{Reactor modeling procedure}

The reactor setup is a typical example of a vertical stagnation flow reactor where gases enter the reactor from the showerhead at the top and spread out onto the substrate as shown in the schematic in figure 4.6.

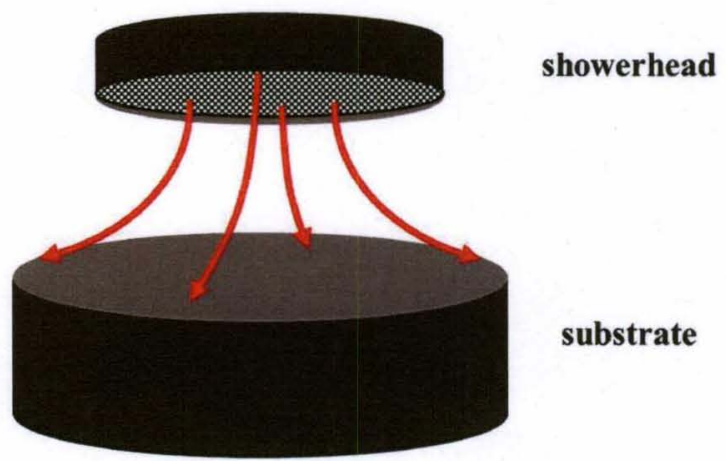

Figure 4.6 Schematic of the vertical stagnation flow system showing the flow pattern of the precursors from the showerhead to the substrate.

Modeling the fluid flow and reactions in the reactor was performed to study the effect of various parameters on the growth process aimed at achieving uniform growth over the largest area possible at reasonably fast growth rates. Commercially available 
Crosslight ProComm software was used for the modeling of fluid flow and material growth rate in the reactor. The software allowed for a 2 dimensional solution to the problem that improves the visualization of the parameters and also helps to understand the effect of various parameters on the growth and flow patterns thus aiding in better tuning of the reactor.

The modeling is done in two parts, the fluid flow and the reactions. The reactor geometry is converted into a mesh to start the process. Since all the parameters are supplied such as pressure, temperature and flow rates, fluid flow equations are solved to calculate the fluid characteristics at each point. Also, knowing the fluid profile, the reaction data is fed into to estimate the mole fractions and deposition rates on the substrate which can be used to study the uniformity of deposition.

\subsubsection{Reactions}

Only GaN reaction was modeled in the current study as the InGaN model has not been studied and the chemistry is not well developed. Also, the InGaN chemistry in the database uses $\mathrm{InN}$ chemistry and adds InN growth rate to $\mathrm{GaN}$ growth rate to get InGaN growth rate which is not the actual $\mathrm{InGaN}$ growth rate.

For any reaction, the forward rate was expressed using modified Arrhenius equation given by

$k_{j}^{f}=A_{j} \mathrm{~T}^{B_{j}} \exp \left[\frac{\left(-E_{j} * 4.182 * \frac{1000}{R}\right)}{T}\right],\left(j=1, \ldots, N_{r}\right.$ 
Where, the parameters $\mathrm{A}, \mathrm{B}$ and $\mathrm{E}$ are provided from experimental or theoretical calculations. $\mathrm{R}$ is the gas constant. The gas phase and the surface reactions for $\mathrm{GaN}$ along with the parameters to calculate the forward rate constants are shown in table 4.2.

Table 4.2 Gas phase and Surface reactions for $\mathrm{GaN}^{160}$

\begin{tabular}{|c|c|c|c|}
\hline Reactions & $\mathbf{A}$ & B & $\mathbf{E}$ \\
\hline \multicolumn{4}{|l|}{ Gas phase reactions } \\
\hline $\mathrm{Ga}\left(\mathrm{CH}_{3}\right)_{3}=\mathrm{Ga}\left(\mathrm{CH}_{3}\right)_{2}+\mathrm{CH}_{3}$ & $3.5 \mathrm{E}+15$ & 0 & $5.95 \mathrm{E}+4$ \\
\hline $\mathrm{Ga}\left(\mathrm{CH}_{3}\right)_{3}+\mathrm{NH} 3=\mathrm{TMG}^{*} \mathrm{NH}_{3}$ & $1 \mathrm{E}+12$ & 0 & 0 \\
\hline $\mathrm{TMG}^{*} \mathrm{NH}_{3} \Rightarrow \mathrm{Ga}\left(\mathrm{CH}_{3}\right)_{3}+\mathrm{NH}_{3}$ & $9.5 \mathrm{E}+9$ & 0 & 19 \\
\hline $\mathrm{TMG}^{*} \mathrm{NH}_{3} \Rightarrow\left(\mathrm{DMG}^{*} \mathrm{NH}_{2}\right)+\mathrm{CH}_{4}$ & $1 \mathrm{E}+13$ & 0 & 32 \\
\hline $3 \mathrm{DMG}^{*} \mathrm{NH}_{2} \Rightarrow\left(\mathrm{DMG}^{*} \mathrm{NH}_{2}\right)_{3}$ & $1 \mathrm{E}+21$ & 0 & 0 \\
\hline$\left(\mathrm{DMG}^{*} \mathrm{NH}_{2}\right)_{3} \Rightarrow 3 \mathrm{GaN}<\mathrm{g}>+6 \mathrm{CH}_{4}$ & $4 \mathrm{E}+15$ & 0 & 60 \\
\hline Surface reactions & $\mathrm{A}$ & B & $\mathrm{E}$ \\
\hline $\mathrm{Ga}\left(\mathrm{CH}_{3}\right)_{3}+\mathrm{SV}<\mathrm{s}>=>\mathrm{Ga}<\mathrm{s}>+3 \mathrm{CH}_{3}$ & 0 & 0 & 1 \\
\hline $\begin{array}{c}\mathrm{TMG}^{*} \mathrm{NH}_{3}+\mathrm{SV}<\mathrm{s}>\Rightarrow \mathrm{Ga}<\mathrm{s}>+3 \mathrm{CH}_{3}+ \\
\mathrm{NH}_{3}\end{array}$ & 0 & 0 & 1 \\
\hline $\mathrm{Ga}\left(\mathrm{CH}_{3}\right)+\mathrm{SV}<\mathrm{s}>\Rightarrow \mathrm{Ga}<\mathrm{s}>+\mathrm{CH}_{3}$ & 0 & 0 & 1 \\
\hline $\mathrm{DMG}^{*} \mathrm{NH}_{2}+\mathrm{SV}<\mathrm{s}>\Rightarrow \mathrm{GaN}<\mathrm{s}>+2 \mathrm{CH}_{4}$ & 0 & 0 & 1 \\
\hline $\mathrm{GaN}<\mathrm{g}>+\mathrm{SV}<\mathrm{s}>=>\mathrm{GaN}<\mathrm{s}>$ & 0 & 0 & 1 \\
\hline $\begin{array}{c}\left(\mathrm{DMG}^{*} \mathrm{NH}_{2}\right)_{3}+3 \mathrm{SV}<\mathrm{s}>=>3 \mathrm{GaN}<\mathrm{s}>+ \\
6 \mathrm{CH}_{4}\end{array}$ & 0 & 0 & 1 \\
\hline $\mathrm{Ga}<\mathrm{s}>+0.5 \mathrm{~N}_{2}=>\mathrm{GaN}<\mathrm{d}>+\mathrm{SV}<\mathrm{s}>$ & $1 \mathrm{E}+10$ & 0 & 32 \\
\hline $\mathrm{GaN}<\mathrm{s}>\Rightarrow \mathrm{GaN}<\mathrm{d}>+\mathrm{SV}<\mathrm{s}>$ & $2 \mathrm{E}+8$ & 0 & 22 \\
\hline $2 \mathrm{GaN}<\mathrm{d}>\Rightarrow \mathrm{N}_{2}+2 \mathrm{Ga}<\mathrm{g}>$ & $2 \mathrm{E}+3$ & 0 & 75 \\
\hline
\end{tabular}


The details of the reactions and the parameters used in the software Procom are

derived from the MIT thesis by T. G. Mihopoulos ${ }^{160}$.

\subsubsection{Modeling procedure}

1. The reactor geometry is created with all dimensions to scale.

2. Each section that requires different meshing (resolution of parameter calculation) is defined separately.

3. A mesh is generated for the geometry where parameters are estimated at each mesh point.

4. Parameters are varied and reaction outputs such as growth rate, temperature profile etc. can be plotted wrt reactor geometry.

The reactor parts are measured and translated into a 2D schematic as all the geometry is assumed to be cylindrical and symmetric about the central axis in the software. All the dimensions are used from figure $4.3 \mathrm{~b}$ which is drawn to scale. Each part of the system has to be defined in the geometry to match the actual system. The model requires inlet, outlet, substrate, hot wall and cold wall conditions that are defined appropriately. However, the difference in the actual reactor and the model is that the inlet is the entire top surface in the model while it is the showerhead in the actual system. The reason for that is that the meshing generates error when the top surface is denoted partially as inlet and partially as a cold wall. 


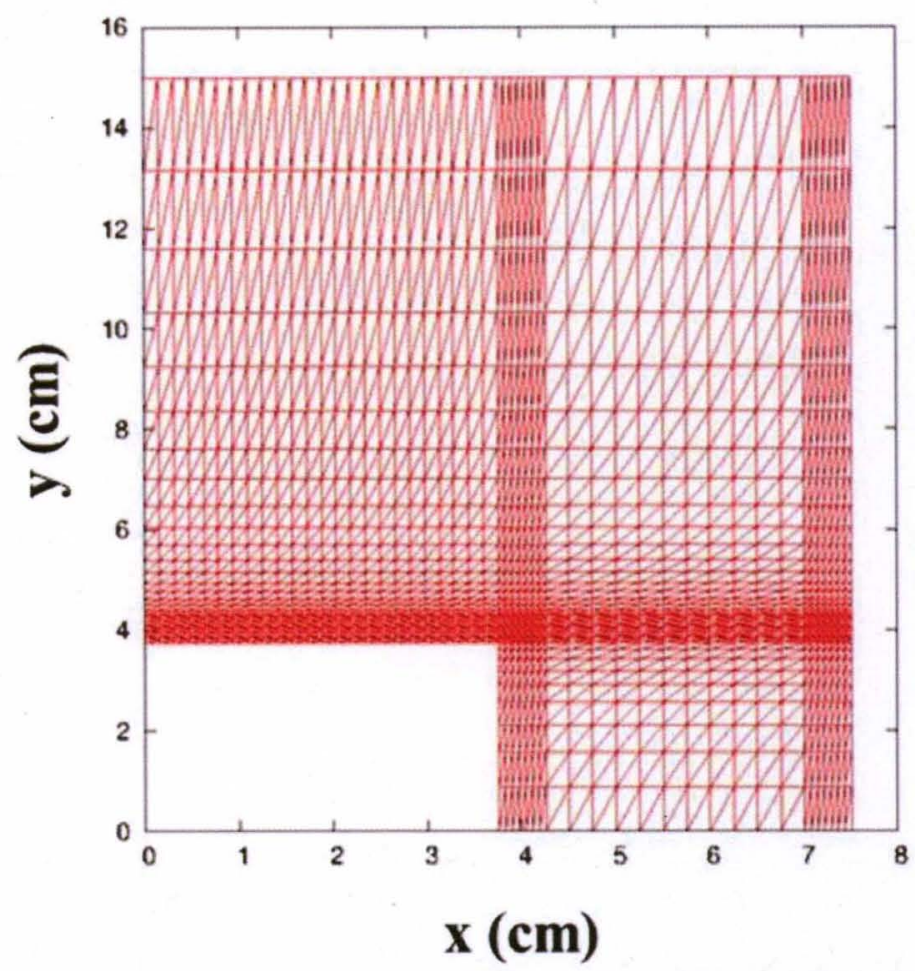

Figure 4.7 Meshing of the symmetric half of the reactor. The regions of interest (close to the substrate and walls) are meshed tightly and the volume is under a relaxed mesh.

Figure 4.7 is the reactor geometry after the mesh generation. The regions of interest i.e. close to the substrate, the walls and the boundary at the inlet and substrate are meshed tightly than the rest of the volume for accurate calculations. In this setup, temperature, pressure and flow rates can be modified without altering the geometry and meshing. However, to study the effect of substrate and showerhead spacing, new geometry and meshing has to be setup.

Table 4.2 is the list of all the parameters used to model the reactor to understand the effect of various parameters on the growth rate and uniformity. 
Table 4.2: List of all the parameters used in reactor modeling

\begin{tabular}{|cc|}
\hline parameter & Value \\
\hline Flow rate $\mathrm{N}_{2}(\mathrm{sccm})$ & 5,15 \\
\hline Pressure (Torr) & $8,80,400$ \\
\hline Temperature (K) & $823,923,1023,1123$ \\
\hline Separation (inch) & $0.5,1.5,3,4.5$ \\
\hline
\end{tabular}

\subsection{Reactor modeling: results}

Growth rate across the substrate from the center to the edge is measured as a function of the various parameters listed in table 4.4, as uniformity and reasonably fast growth rate is critical in evaluation of any growth process. The quality of the crystal cannot be ascertained which can only be quantified experimentally.

Each $3 \mathrm{~d}$ plot contains growth rate as a function of temperature and the position from the center of the substrate. A representative plot is shown in figure 4.8 with the axes labeled. All plots have the same arrangement of parameters with Temperature (K) in " $\mathrm{x}$ ", distance from the substrate center $(\mathrm{cm})$ on " $y$ " and growth rate $(\mu \mathrm{m} / \mathrm{hr})$ on the " $\mathrm{z}$ " axis. 


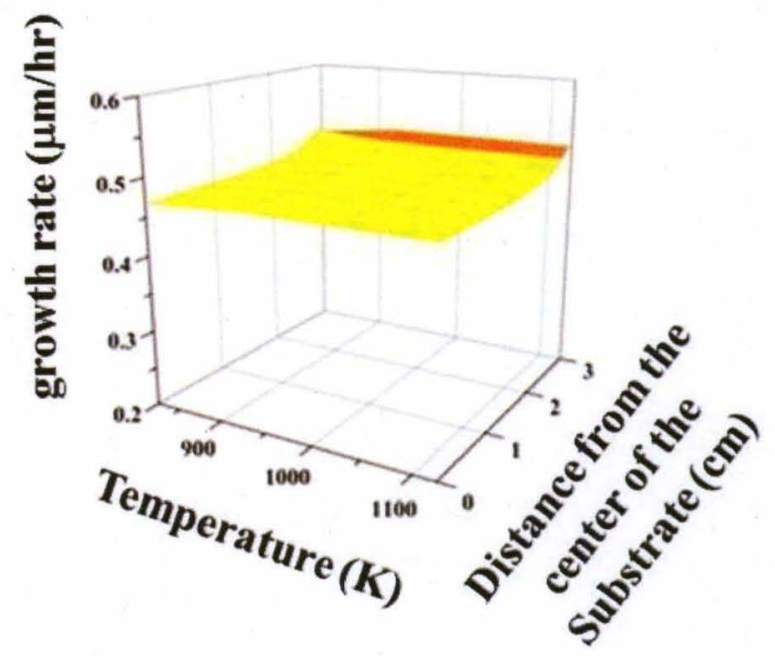

Figure 4.8 Representative 3-d plot showing the dependence of growth rate as a function of the parameters described in table 4.2. All the plots have temperature in the $\mathrm{x}$ and distance from the center of the substrate on the $y$ axis.

\subsubsection{Effect of precursor flux}

This is very intuitive as the growth rate is expected to increase with increasing precursor flux (flow rate). Modeling was run at $5 \mathrm{sccm}$ and $15 \mathrm{sccm} \mathrm{N} \mathrm{N}_{2}$ flow rates to ascertain the difference. It can be seen in figure 4.9 that there is almost a linear dependence of growth rate on the flux. 
$5 \mathrm{sccm} \mathrm{N}_{2}, 8$ Torr, 0.5 inch

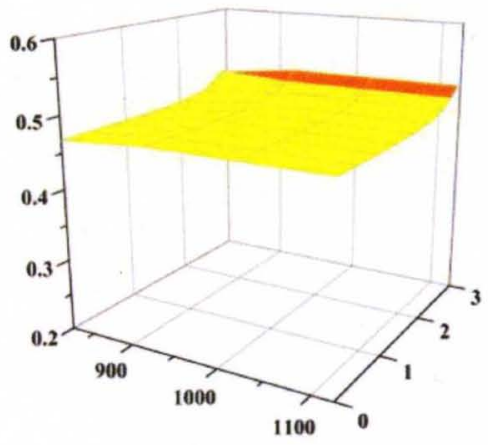

$5 \mathrm{sccm} \mathrm{N}, 400$ Torr; 4.5 inch

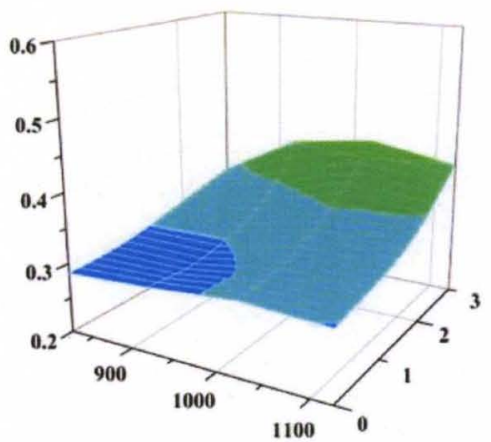

15 sccm $\mathrm{N}_{2}, 8$ Torr, 0.5 inch

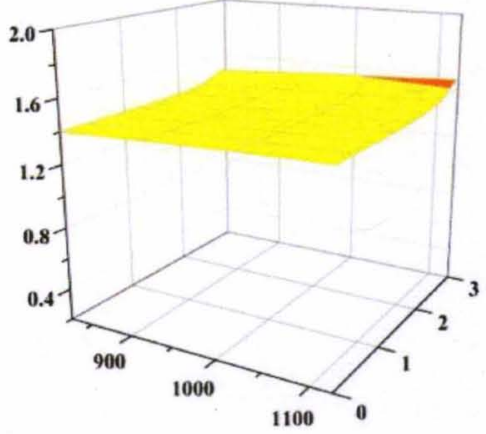

$15 \mathrm{sccm} \mathrm{N}_{2}, 400$ Torr, 4.5 inch

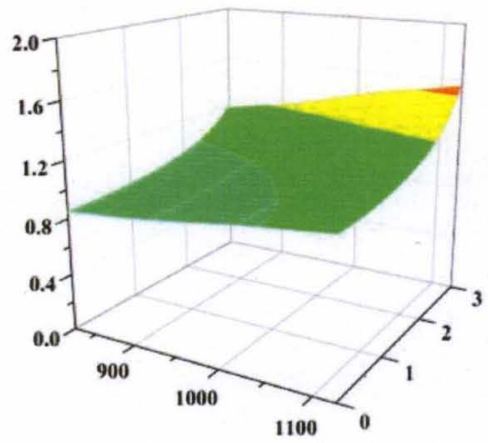

Figure 4.9: Comparison of growth rates of $\mathrm{GaN}$ as a function of material flux. The flow rate of carrier gas $\mathrm{N}_{2}$ was increased three times from $5 \mathrm{sccm}$ to $15 \mathrm{sccm}$. A linear change in growth rate is observed.

This behavior is consistent at different substrate distances, separation distances, temperatures and pressures. Flow rate or flux is a measure of the number of reactive species in the system. 


\subsubsection{Effect of substrate temperature}

Each plot includes the effect of temperature on the growth rate and hence uniformity of growth. The consistent observation is that the growth rate is almost constant with temperature. There is a very small increase in growth rate with temperature which is pronounced at higher pressures and at larger distances away from the center of the substrate as shown in figure 4.10 . The effect could also be pronounced due to the variation in the effect of other parameters at different pressures and substrate positions.

Temperature controls the rate of reaction as well as the adsorption and desorption dynamics of the adatoms. It could be that the distribution of species affects the adatom distribution and migration that can affect the growth rate.

\subsubsection{Effect of pressure}

Effect of pressures in the range of 8 Torr to 400 Torr was studied. A decrease in growth rate is observed with increasing pressure. It could be due to the fact that the decomposition reaction of the species into the adatoms is hampered at higher pressures. Higher pressures could also change the flow patterns altering the distribution of species in the chamber. Figure 4.10 is a compilation of the growth rate modeled to show the effect of reactor pressure. The reduction is significant from 8 Torr to 80 Torr and the reduction is very small from 80 Torr to 400 Torr indicating a non-linear pressure dependence of the decomposition reactions. 
$5 \mathrm{~N}_{2}, 8$ Torr, 0.5 inch

$5 \mathrm{~N}_{2}, 8$ Torr, 4.5 inch
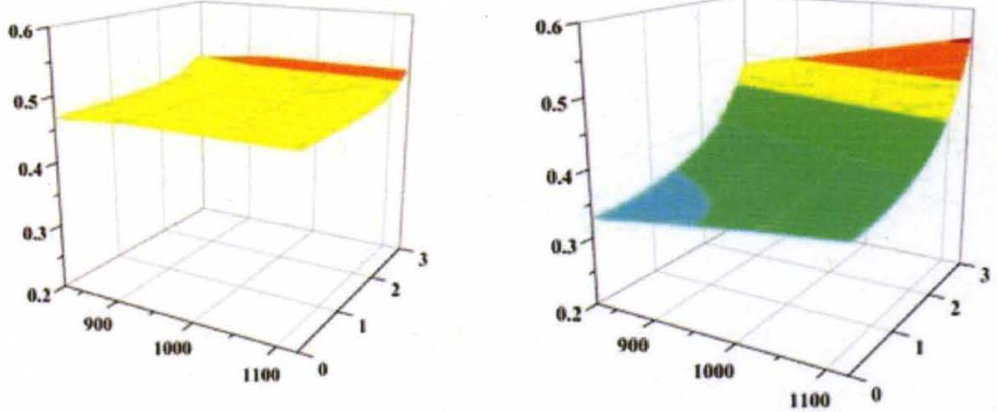

$5 \mathrm{~N}_{2}, 80$ Torr, 0.5 inch
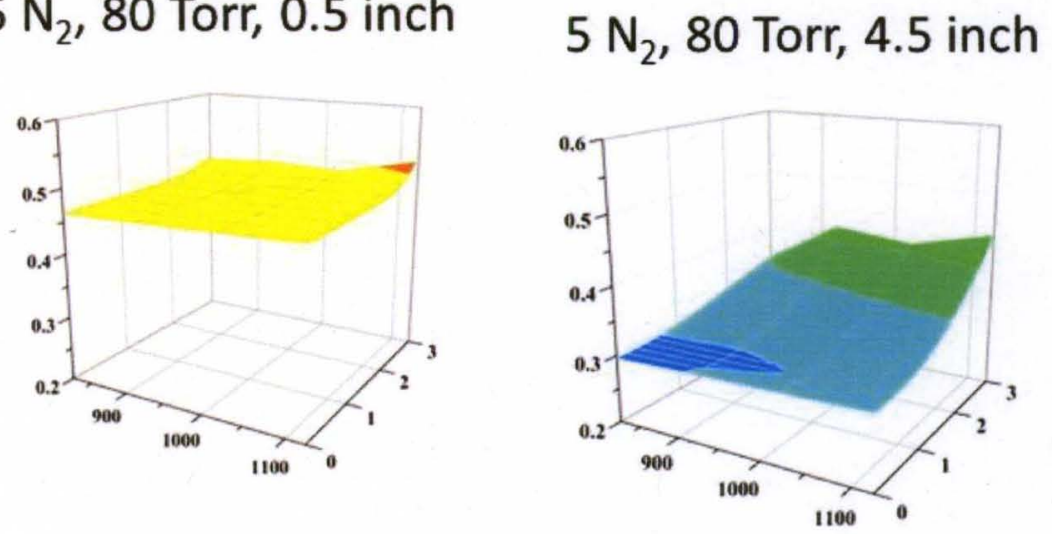

$5 \mathrm{~N}_{2}, 400$ Torr, 0.5 inch
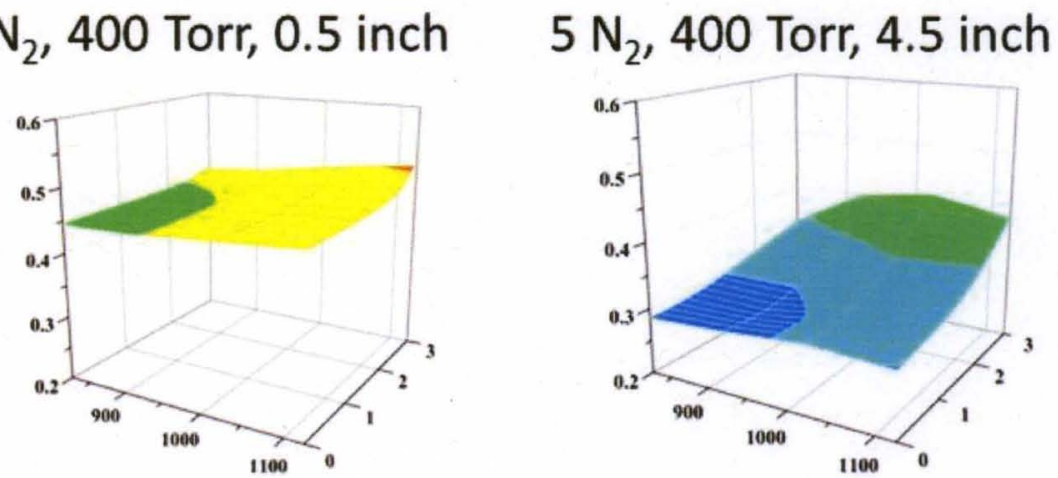

Figure 4.10 Comparison of growth rates as a function of temperature. The growth rate shows a very little increase with temperature (almost constant at small separation distances) with the effect being pronounced at higher pressures and at larger distances from the center of the substrate. 


\subsubsection{Effect of separation of the substrate and showerhead}

This is perhaps the most critical reactor parameter that requires extensive tuning to achieve better uniformity as the rest of the parameters are process parameters that control the crystal quality and composition but the separation actually can be used to achieve better uniformity as well as control over composition to a certain extent.

Figure 4.11 is a compilation of the modeling results to understand the effect of separation distance on the growth. Growth rate is highest and most uniform growth is obtained at 0.5 inch separation. As the separation increases, the growth rate at the center of the substrate drops by $50 \%$. However, the growth rate on the ends of the substrate actually remains constant. The reduction at the center and the high growth rate at the ends of the substrate could be due to the distribution of the precursors with distance. As the separation distance increases, the flow can form a channel from the inlet at the showerhead to the pumping line which moves the precursor away from the center of the substrate to the ends maintain the growth rate at the ends with a reduction at the center. A very small reduction in growth rate at the center of the substrate is observed when the distance is reduced from 1.5 inch to 4.5 inch which indicates that the channel flow is created as soon as the separation is increased from 0.5 inch to $1.5 \mathrm{inch}$. 
$15 \mathrm{~N}_{2}, 8$ Torr, 0.5 inch $15 \mathrm{~N}_{2}, 400$ Torr, 0.5 inch
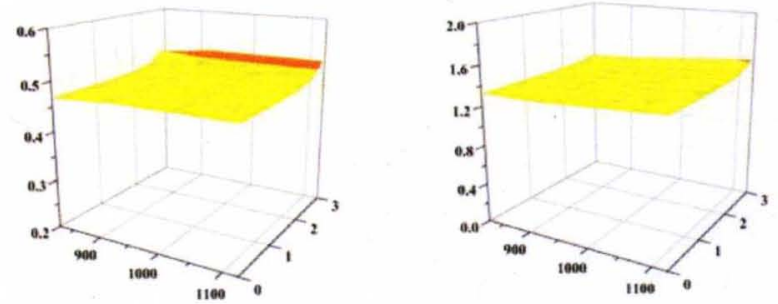

$15 \mathrm{~N}_{2}, 8$ Torr, 1.5 inch

$15 \mathrm{~N}_{2}, 400$ Torr, 1.5 inch
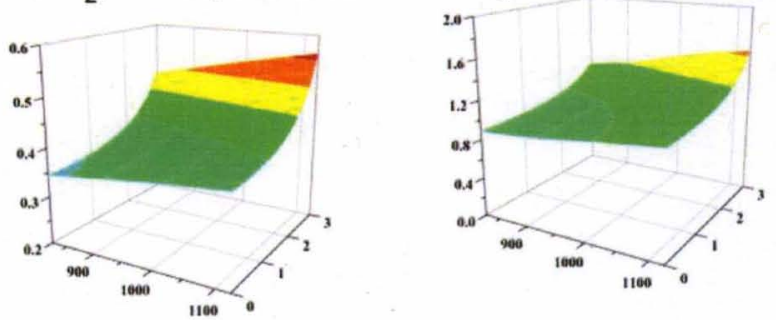

$15 \mathrm{~N}_{2}, 8$ Torr, 3.0 inch

$15 \mathrm{~N}_{2}, 400$ Torr, 3.0 inch
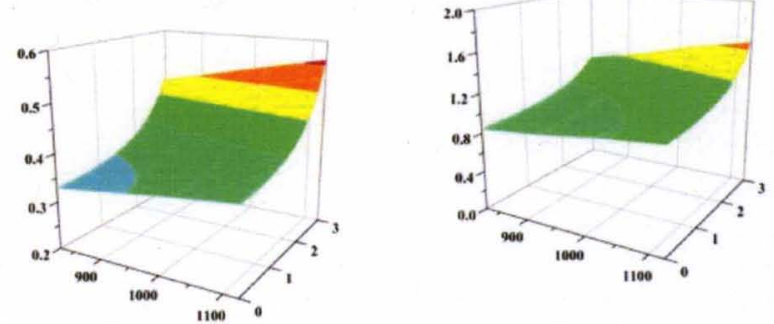

$15 \mathrm{~N}_{2}, 8$ Torr, 4.5 inch

$15 \mathrm{~N}_{2}, 400$ Torr, 4.5 inch
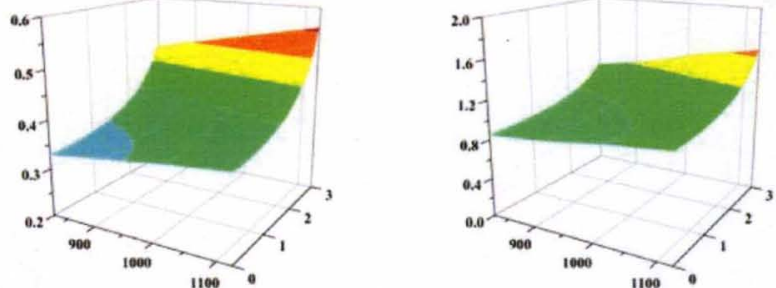

Figure 4.11: Effect of substrate showerhead separation on the growth rate and uniformity of deposition. The growth rate drastically drops from 0.5 inch to $1.5 \mathrm{inch}$ at the center but is almost constant at the ends of the substrate. 
The effect of separation of the substrate and the showerhead can be ascertained by the difference in growth rates observed as shown in figure 4.12. Growth for the same duration is about $50-100 \mathrm{~nm}$ when the separation is 3 inches while the growth is more uniform and thicker (150-200 nm) clearly showing that closer spacing leads to a faster growth rate. The underlying nanowires in both cases were about $15-20 \mathrm{~nm}$ in diameter.

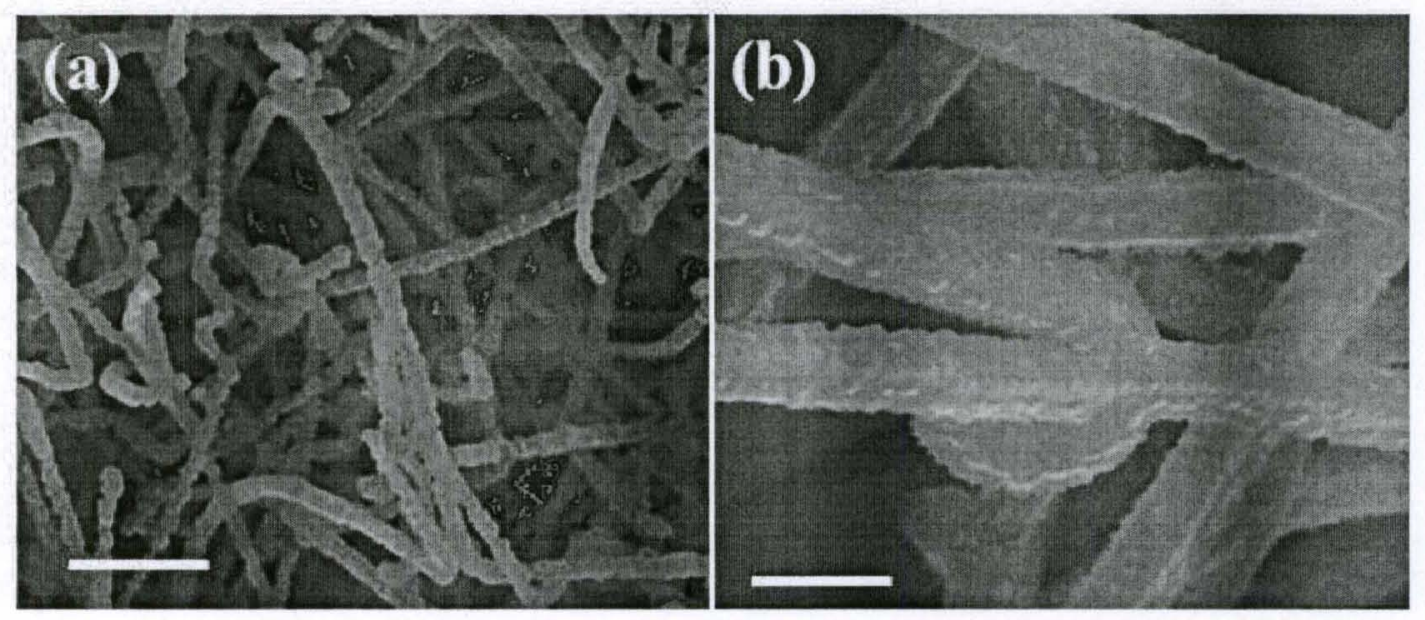

Figure 4.12 SEM images of the InGaN growth on $\mathrm{GaN}$ nanowires showing the effect of substrate and showerhead separation on the growth rate. The growth at a) 4 inch separation is only $100-125 \mathrm{~nm}$ in 15 minutes while the growth at b) 1 inch separation is about $250-350 \mathrm{~nm}$ thick for the same duration. The scale bar in the images is $500 \mathrm{~nm}$. 
Experimentally, it was observed that a 2 inch separation and 100 Torr pressure was the best for controlled uniform growth. If the model was used, a 0.5 inch separation and 8 Torr would be the best conditions. However, the actual reactor has to be modeled to use the parameters in designing experiments.

Figures 4.13 and 4.14 are the complete modeling data for various pressure, temperature, separation and flow rate combinations. Figure 4.13 has data for $5 \mathrm{sccm} \mathrm{N}_{2}$ carrier flow rate and figure 4.14 includes the results for $15 \mathrm{sccm} \mathrm{N} \mathrm{N}_{2}$ flow rate. The horizontal axis has data from three pressures $(8,80$ and 400 Torr) and the vertical axis has data from four separations $(0.5,1.5 .3$ and 4.5 inch). 


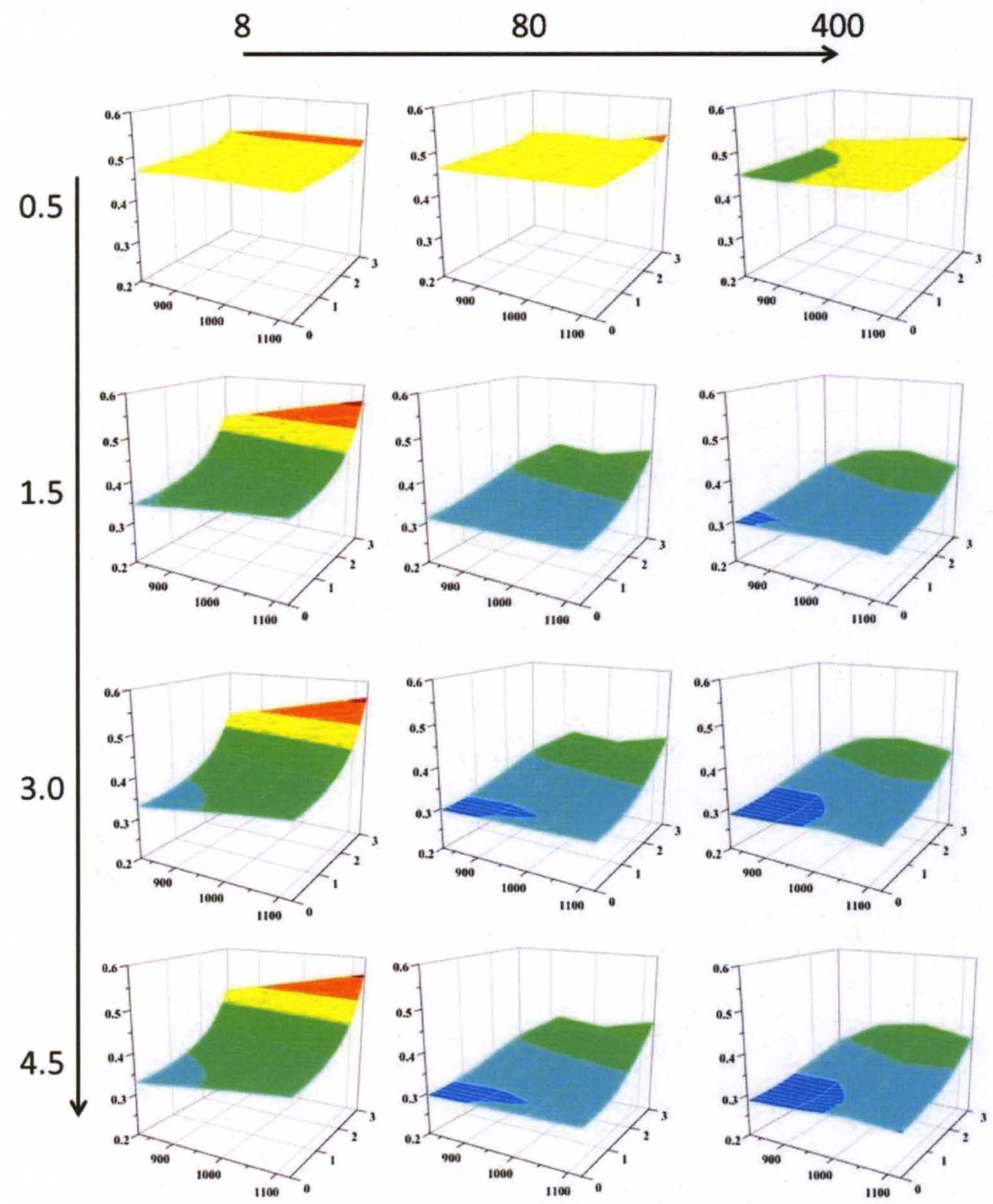

Figure 4.13 Growth rate plots at different separation, pressure and temperature for $5 \mathrm{sccm}$ $\mathrm{N}_{2}$ flow. 


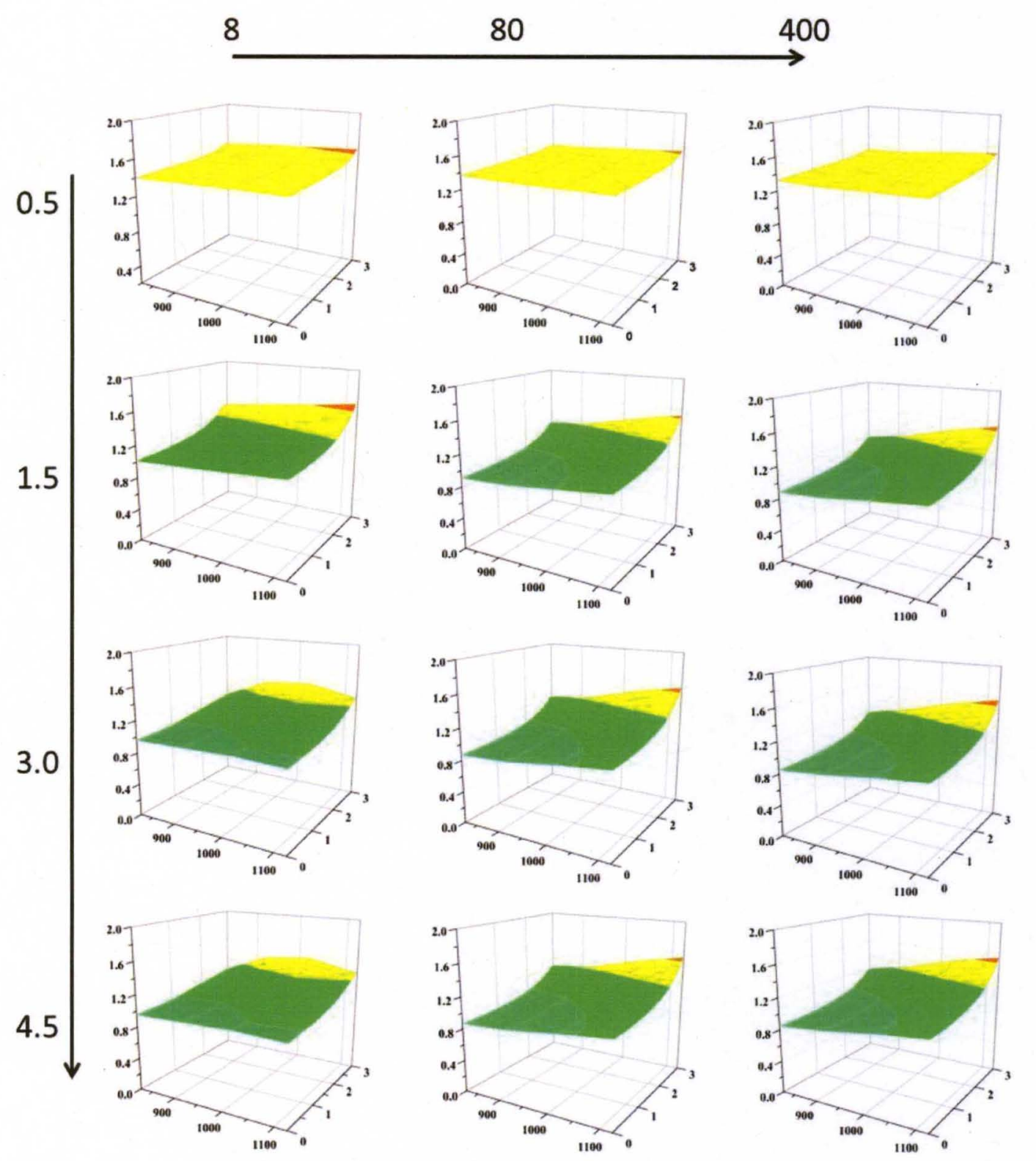

Figure 4.14 Growth rate plots at different separation, pressure and temperature for 15 sccm $\mathrm{N}_{2}$ flow. 
The model used for the reactor simulation is not an exact model of the reactor. This inlet port and the pump port are not the right sizes which could lead to a huge variation in the actual growth in the reactor. Some of the observations actually point to the fact. If the inlet is only via the showerhead, the flow would impinge on the substrate with uniformity at larger distances that that has been modeled as channeling would be reduced. The overall growth rate would also be reduced which would be an accurate modeling of the system as well.

Also, the system has been modeled for GaN film growth. InGaN growth would require InGaN reaction chemistry which has not been studied and need in situ measurements of the species to identify all the adatoms. 


\section{CHAPTER 5}

\section{SYNTHESIS OF GaN AND III-ANTIMONIDE NANOWIRES}

\subsection{Introduction}

This chapter includes the results and analysis of

1. III-Sb nanowire growth to understand the nanowire growth mechanism and the role of equilibrium solubility in controlling nanowire growth.

2. Orientation controlled synthesis of GaN nanowires on different substrates.

\subsection{Controlling nanowire growth mode}

There is a lot of interest in III-V semiconductor nanowires for possible applications in power electronics and opto-electronic devices ${ }^{161,162}$. Development of nanowire synthesis techniques is critical for the creation of optimal device structures. In this regard, control and understanding of nanowire growth mechanism is of paramount importance. 
Traditionally, catalyst assisted VLS growth has been the most widely used method for semiconductor nanowire synthesis ${ }^{163,164,165}$. In this method (illustrated in Fig 5.1), a foreign metal droplet is used as a catalyst which acts to crack the gas phase precursors and dissolve the growth species forming a eutectic alloy. Further dissolution leads to supersaturation causing precipitation. Precipitation has been observed to occur at the substrate droplet interface which causes the catalyst tip led growth of nanowires as shown in the schematic in fig 5.1.

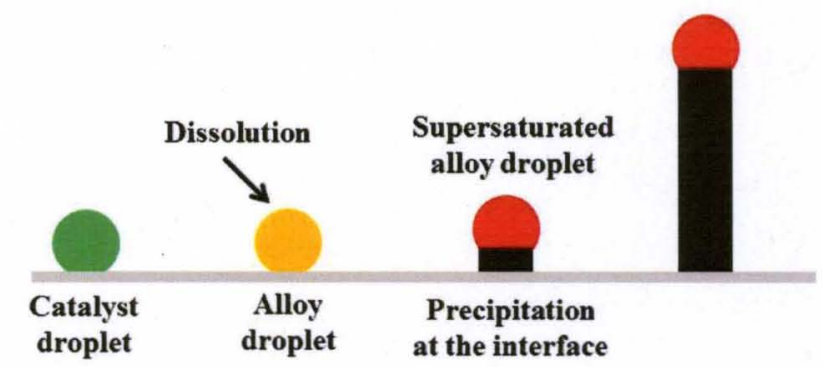

Figure 5.1 Schematic illustrating the process sequence of catalyst assisted VLS growth. The catalyst droplet causes the cracking of the gas phase species and dissolution of the growth species and further dissolution causes supersaturation. Precipitation occurs at the liquid substrate interface leading to the tip led growth of a nanowire.

Precise control over the nanowire morphology and epitaxy ${ }^{165}$ has been achieved via catalyst assisted process, the translation of this process into mainstream electronics/device assembly is an issue due to the detrimental effects of the catalyst metal on the semiconductor properties. Hence, there is a tremendous interest in catalyst free synthesis of III-V nanowires. There have been various reports of such synthesis using various methods such as $\mathrm{MOCVD}^{166}, \mathrm{MBE}^{167}$ and $\mathrm{HVPE}^{168}$. GaN nanorods have also been synthesized on masked substrates via $\mathrm{MOCVD}^{169}$ without any catalyst. MOCVD 
growth of indium tip led InP nanowires ${ }^{170,171,172}$ via MOCVD has also been reported. However, most of these methods fail to address the underlying mechanism controlling the growth and how to control the growth process.

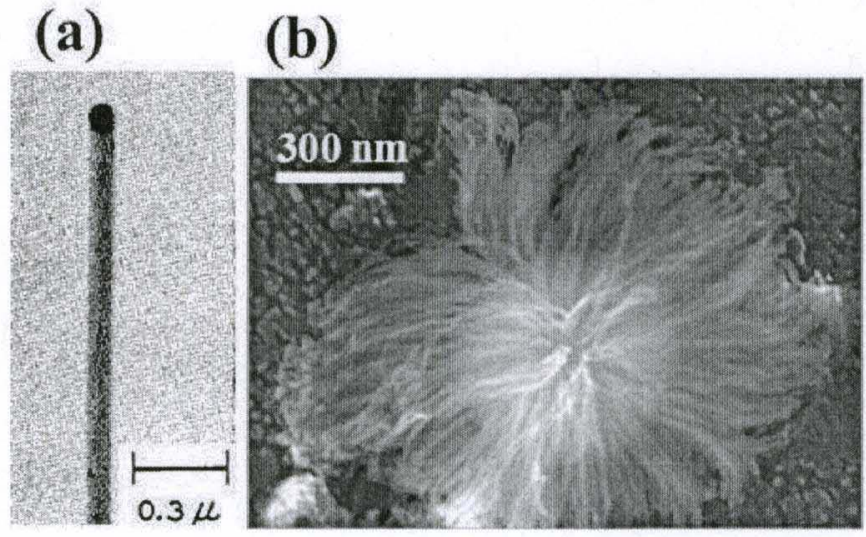

Figure 5.2: SEM micrographs of the two different nanowire growth modes. a) Tip led growth of silicon nanowires. b) Bulk nucleation of bismuth nanowires out of one gallium droplet.

\subsubsection{Low melting metal mediated nanowire growth}

Low melting metals (Gallium/Indium) have also used as mediators for the catalyst free synthesis of $\mathrm{GaN}^{173}, \mathrm{InN}^{174}, \mathrm{GaSb}^{175},{ }^{176}$ and $\mathrm{InSb}^{175},{ }^{176}$ nanowires. The metal droplet is not foreign but is one of the constituent materials, hence the term "self-catalysis". Here, apart from the generally observed tip-led growth shown in fig 5.2a, high density of nanowires has been observed to grow out of one droplet as shown in fig $5.2 \mathrm{~b}$.

The observed bulk nucleation growth mode has been attributed to the very low solubility eutectic of the growth species in the mediating droplet. Fig 5.3 shows a comparison between the Ga-N and Au-Si phase diagrams which clearly shows the very 
different eutectic composition. It is $<10^{-6}$ at $\%$ for Ga-N while the composition very large $\sim 20 \%$ for Au-Si system. The exact translation of the role of eutectic composition needs to be ascertained to have a better understanding of the nucleation process.

Almost all of the phase diagrams have a common feature that if the eutectic composition is small, the liquidus line is very steep (figure 5.3a) and the ratio $\mathrm{C} / \mathrm{C}^{*}$ is orders of magnitude higher than that in the case of a phase diagrams with large eutectic compositions (figure 5.3b). Critical nuclei size estimations indicate that it is $<20 \mathrm{~nm}$ for Ga-N system which will favor bulk nucleation out of a micron sized droplet while it is in microns for Au-Si system which will favor a tip led growth out of a sub-micron size droplet. Therefore, the role of critical nuclei size (the factors that govern the same) i.e. equilibrium solubility and hence the growth mode needs to be understood better.

(a)

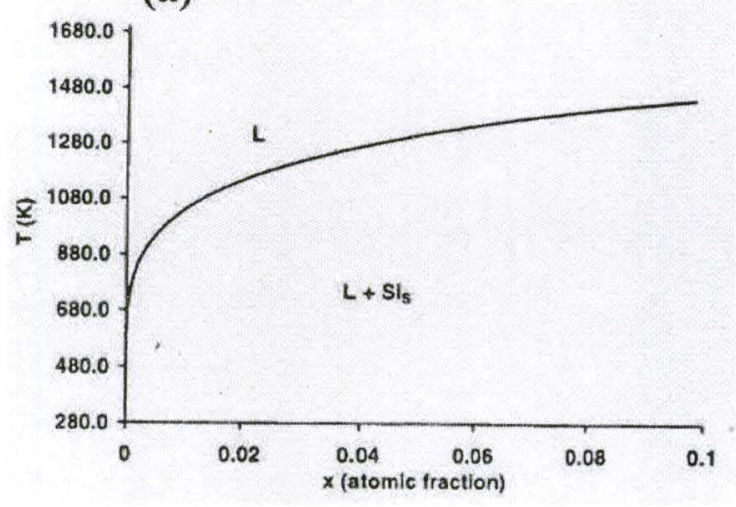

(b)

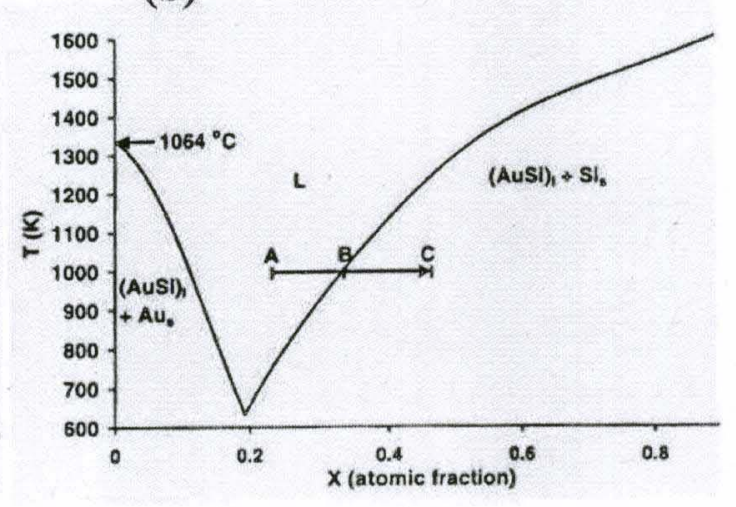

Figure 5.3 Binary phase diagrams of a) Ga-N and b) Au-Si depicting the very small and large eutectic compositions in the two systems.

Towards this objective, Ga-Sb system is chosen as a model system. Figure 5.4 shows the binary phase diagram of the Ga-Sb system. The unique feature of the phase 
diagram is the coexistence of two eutectics with different compositions at the extremes of the phase diagram. Region 1 is the eutectic with the very low solubility eutectic of $\mathrm{Sb}$ in $\mathrm{Ga}\left(<10^{-5}\right)$. On the other hand, region 2 is the high solubility eutectic of $\mathrm{Ga}$ in $\mathrm{Sb}(\sim$ $11 \%$ ). This gives an opportunity to study the role of equilibrium solubility on the nanowire growth process, eliminating any material specific discrepancies. Bulk nucleation and growth of GaSb nanowires, for the case of vapor transport of antimony source over gallium coated substrate (excel gallium conditions) at conditions close to regions 1 and 2 has already been reported ${ }^{175}$.

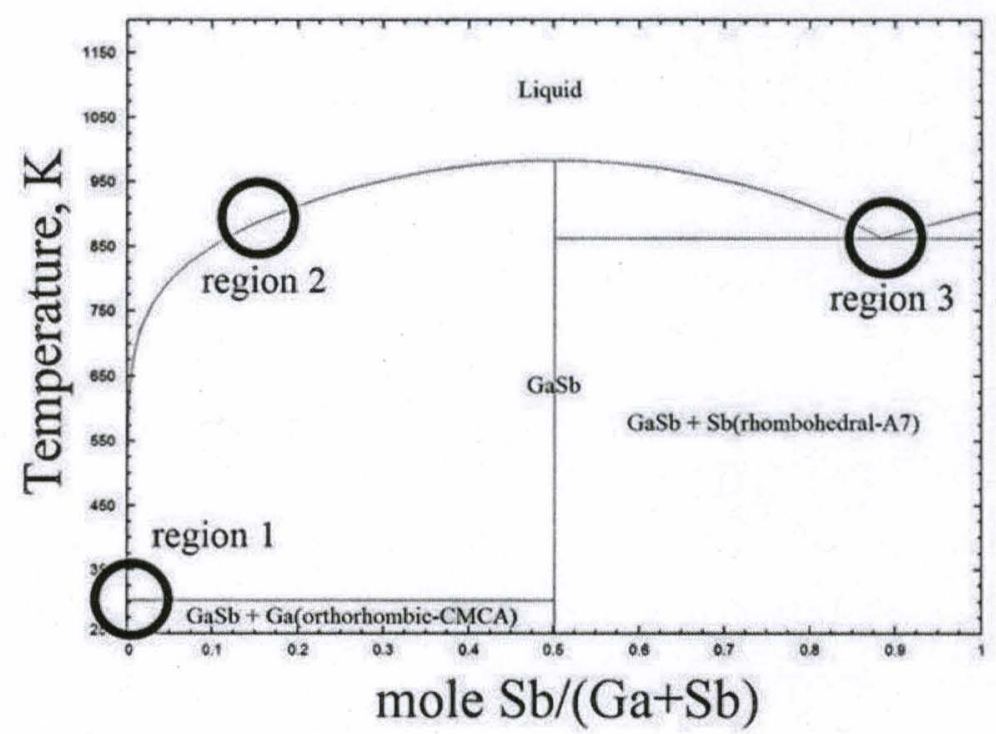

Figure 5.4 Phase diagram of the Ga-Sb system. The three regions are marked for being very different in eutectic composition and equilibrium solubility to study the role of the same in controlling nanowire growth mode. Region 1 and region 3 are the two eutectics at excess gallium and excess antimony regions. Region 2 is marked as the range for gallium tip led growth. 
It is hence useful to study if antimony can mediate $\mathrm{GaSb}$ nanowire growth analogous to gallium when operating under the conditions that lie within region 3 . For this, the experimental setup had to be designed to have excess antimony conditions. Gallium and antimony were used as the source materials. $\mathrm{SbCl}_{3}$ was not used as the antimony source as it would be a little tricky to crease excess antimony conditions with the vapor phase precursor. Antimony has a very high vapor pressure and the substrate should have a large excess of antimony to account for the losses due to evaporation at the synthesis temperatures. The experimental setup has been described in section 3.2.2. 


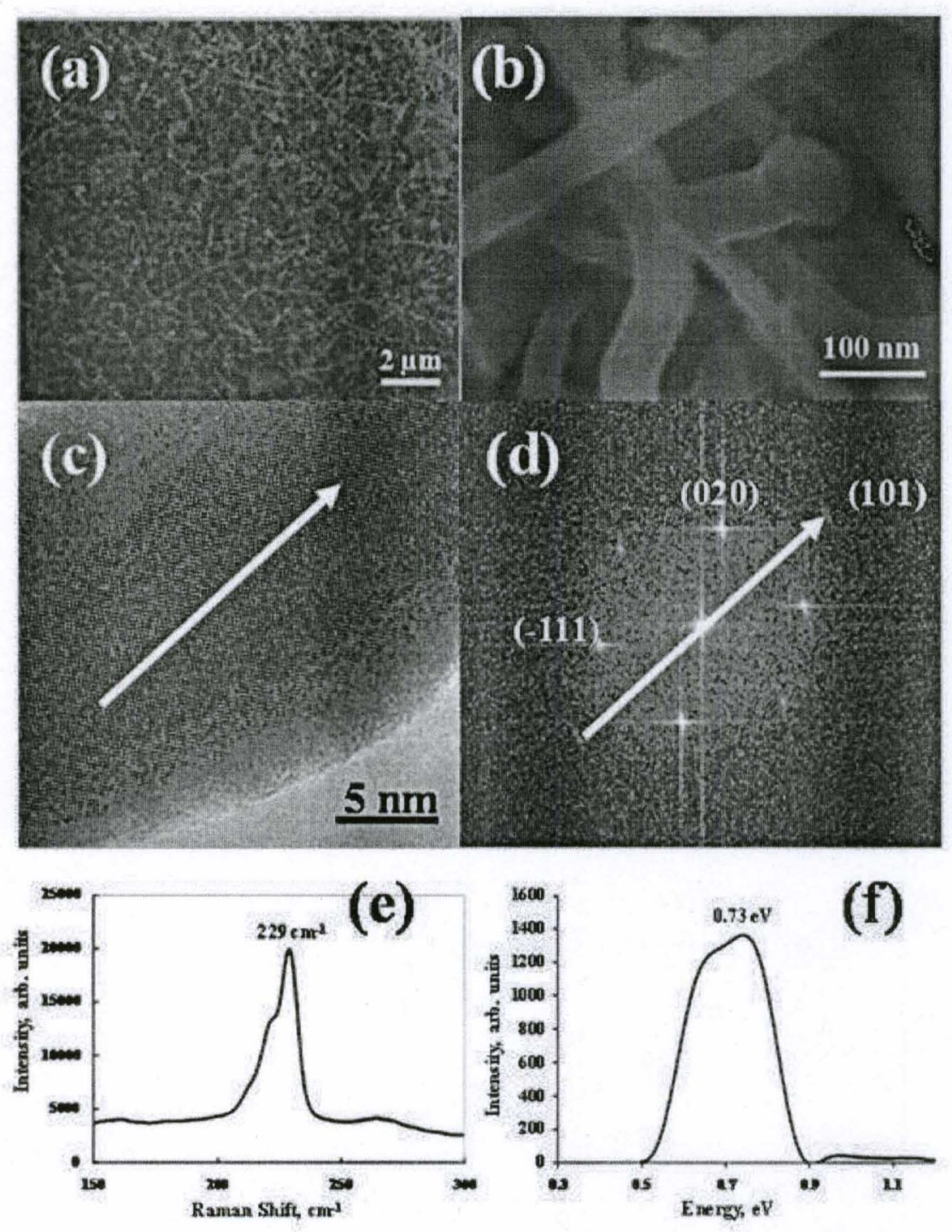

Figure 5.5 a) SEM micrograph of the GaSb nanowires synthesized on quartz substrates under antimony rich conditions. b) Magnified view of the GaSb nanowires showing the tip. c) High resolution TEM image of the nanowire showing the single crystalline nature of the nanowire. d) FFT pattern of the nanowire showing the $<101>$ growth direction. e) Raman spectrum of the GaSb nanowires showing the characteristic $229 \mathrm{~cm}^{-1}$ peak. f) NIR PL emission from the GaSb nanowires showing peak at $0.73 \mathrm{eV}$ the characteristic band edge emission of $\mathrm{GaSb}^{176}$. 
At the start of the heating process, antimony evaporates from the crucible and deposits on the substrate placed on the crucible. This creates the excess antimony condition required. However, there is also a constant loss of antimony when the system is heated to the desired temperature $735 \mathrm{C}$. It is very important to have excess antimony at this stage on the substrate. Now, gallium vapors reach the substrate dissolve into antimony and $\mathrm{GaSb}$ precipitates into nanowires. Figure 5.5a is the SEM micrograph of $\mathrm{GaSb}$ nanowires obtained from this experiment. The nanowires are $30-50 \mathrm{~nm}$ in diameter and microns long. The nanowires were observed to have tips indicating that it was a tip led growth process. The single crystalline nanowires grow in the $<10-11>$ direction as shown in the HRTEM images in figures $5.5 \mathrm{c}$ and $\mathrm{d}$ respectively. The material is confirmed to be GaSb as the Raman spectrum has the peak at $229 \mathrm{~cm}^{-1}$. Also, the band edge emission in the near infrared at $0.73 \mathrm{eV}$ is a characteristic of GaSb material system.

After confirming the nanowires to be of pure phase single crystalline $\mathrm{GaSb}$ nanowires grown via a tip led process, the next step is to investigate the tip composition to understand the mechanism of growth. Figure 5.6 is the TEM EDS line scan along the tip and the nanowire. It is clear that the nanowire contains a uniform distribution of $\mathrm{Ga}$ and $\mathrm{Sb}(\mathrm{GaSb})$ as shown in other characterization data in figure 4.4. However, the interesting thing to note is that the tip contains excess antimony and very little gallium. 

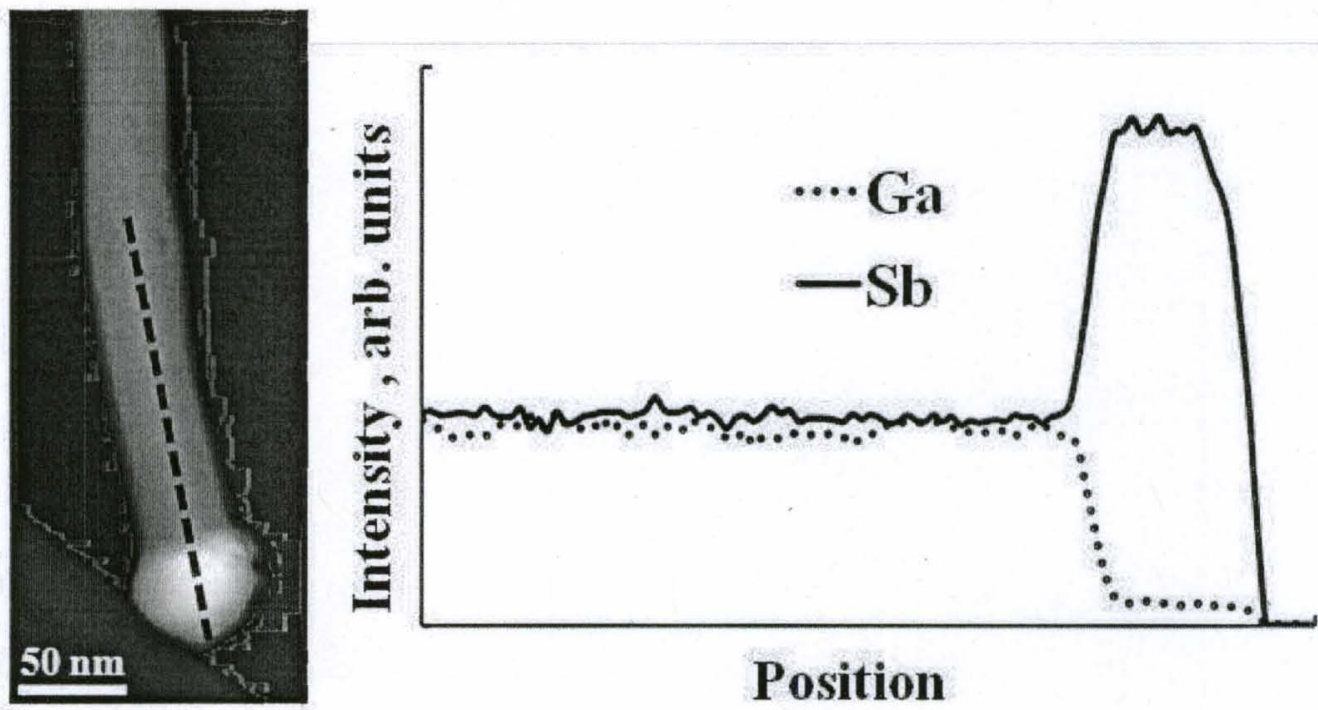

Figure 5.6 TEM EDS line scan along the GaSb nanowire and the tip. The nanowire is $\mathrm{GaSb}$ with equal and uniform distribution along the length while the tip is almost pure antimony and very little residual gallium in the same proportion as the eutectic composition.

The amount of gallium is similar to that would be left in the alloy due to the $11 \%$ eutectic composition. Also, analogous to the observed bulk nucleation in the case of excess gallium and antimony in the vapor phase, excess antimony should have resulted in bulk nucleation of GaSb nanowires out of an antimony droplet. This result is an important step in understanding the growth modes. Further, the reason for the tip led growth rather than bulk nucleation has to be investigated. Critical nuclei size is defined as the diameter of the largest possible stable nuclei precipitating out of a supersaturated solution and is estimated by 


$$
d_{c}=\frac{4 \sigma \Omega}{R T \ln \left(\frac{C}{c^{*}}\right)}
$$

$\Omega$ is the molar volume (cc/mol) of the nucleating material, $\sigma$ is the liquid-crystal interfacial energy $\left(\mathrm{J} / \mathrm{m}^{2}\right), R$ is gas constant $(\mathrm{J} / \mathrm{mol}-\mathrm{K}), \mathrm{T}$ is the system temperature $(\mathrm{K}), \mathrm{C}$ is the concentration of species in the supersaturated droplet governed by the operating conditions, $\mathrm{C}^{*}$ is the equilibrium concentration which is determined from the liquidus line of the phase diagram at the synthesis temperature. The ratio of the concentrations is the factor that controls the nuclei size to a large extent. The estimation of the interfacial energy is the critical part in these calculations. Interfacial energy can be calculated using equation 5.2

$$
\cos \theta=\frac{\gamma^{s v}-\gamma^{s L}}{\gamma^{L V}}
$$

Where $\theta$ is the contact angle, $\gamma$ is the interfacial energy between solid (s), liquid (L) and vapor (v) phases. The sv and Lv interfacial energies are considered as surface energies of the respective solid and liquid phases. The surface energies of $\mathrm{Ga}^{177}$ and $\mathrm{Sb}^{178}$ are available in literature. The interfacial energy of Ga-GaSb system has been estimated to be $1.14 \mathrm{~J} / \mathrm{m}^{2}$ for gallium rich conditions. Since the Ga-GaSb and $\mathrm{Sb}-\mathrm{GaSb}$ systems are nonwetting $(90<\theta<180$ ), a representative number of 160 (close to completely non-wetting) is used for the calculations. The surface energy of GaSb is estimated from equation 2 using gallium as the liquid and $\mathrm{GaSb}$ as the solid phase at $600 \mathrm{C}$. Comparison of the variation of critical nuclei size with antimony compositions is estimated in figure 5.7 for region 1 (Ga-GaSb system at 300C) and region 2 (Ga-GaSb system at 600C) considering gallium as the liquid and $\mathrm{GaSb}$ as the solid phase. In region 3, antimony is the liquid 
phase and $\mathrm{GaSb}$ is the solid phase as antimony is the phase mediating the growth of nanowires.

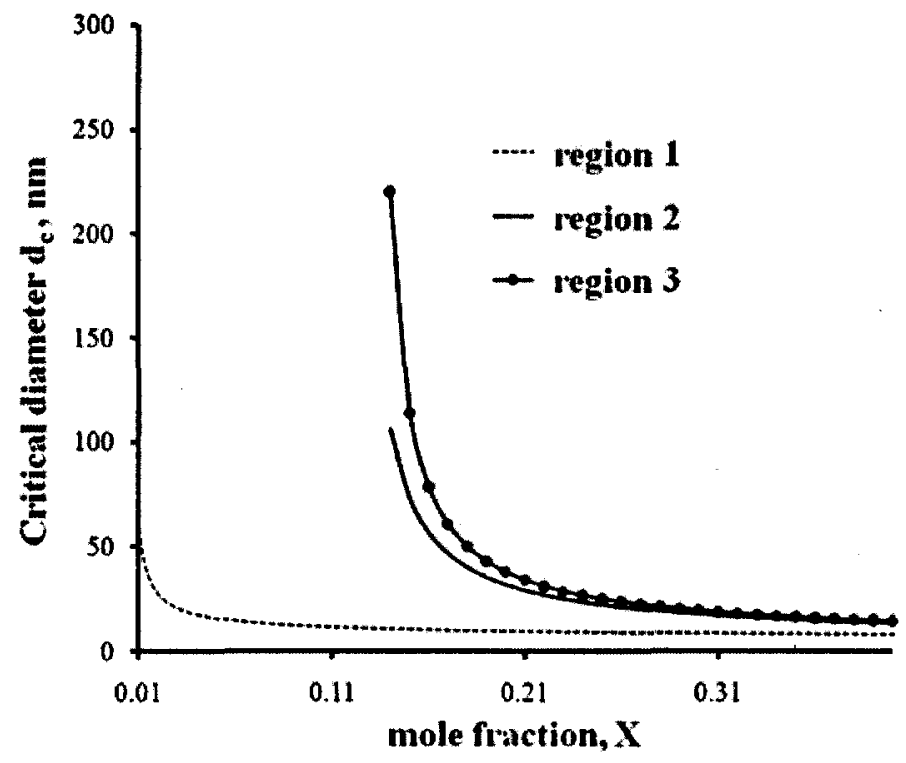

Figure 5.7 Estimated critical nuclei diameters for GaSb system for various antimony compositions. Regions 2 and 3 with the large equilibrium solubility have large critical nuclei sizes and region 1 with low solubility has much smaller critical nuclei size.

The diameter of the synthesized GaSb nanowires in figure $5.5 \mathrm{~b}$ is in the same order of magnitude as the antimony tip which matches reasonably well with the critical nuclei size estimations of around $100-150 \mathrm{~nm}$. The size of the mediating droplet is the difference in the observed antimony tip-led and the bulk nucleation out of a gallium droplet. Since the critical nuclei size is orders of magnitude smaller than the droplet, bulk nucleation is favored whereas when the critical nuclei size is in the same order of magnitude of the droplet, tip led growth is observed. 
If the proposed relation between the critical nuclei size and growth mode is true, gallium tip led growth of $\mathrm{GaSb}$ nanowires should be possible under excess gallium conditions with antimony being supplied in vapor phase for conditions that maintain the system in region 2 . The experimental setup is described in section 3 . The thin coating of gallium on the substrate breaks up into droplets as gallium has very low melting point. Figure 5.8 is the SEM EDS elemental map of a GaSb nanowire with a gallium tip led growth.

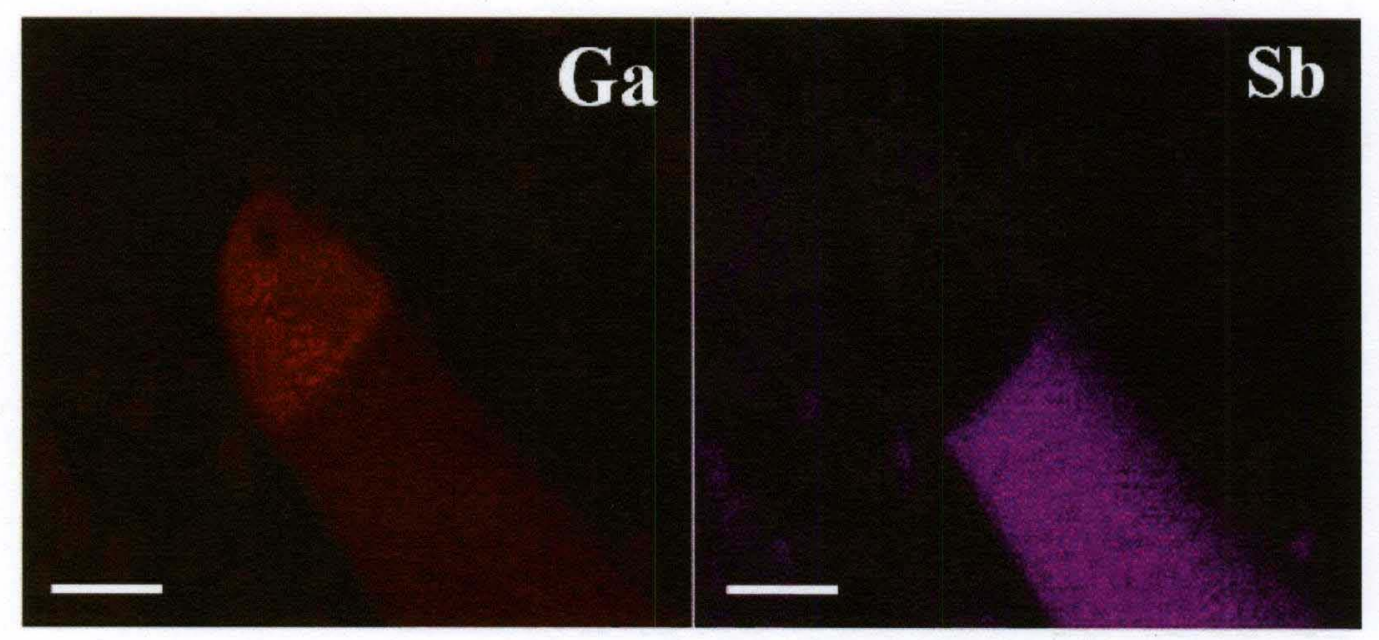

Figure 5.8 SEM EDS elemental map of GaSb nanowire. The absence of antimony at the tip and it showing up in the gallium map is a clear indication of the tip being pure gallium. The scale bar is $1 \mu \mathrm{m}$.

The tip can be clearly identified in gallium map while it is completely absent in the antimony map indicating a pure gallium tip led GaSb nanowire growth. Here, the critical nuclei size is in the order of $200 \mathrm{~nm}$ while the droplet is of the same size leasing to tip led growth. This corroborates the earlier proposed claim that equilibrium solubility 
can be used to design growth conditions to control the nanowire growth mode. The process can be tuned better by the controlled supply of the source materials via MOCVD rather than the use of bulk materials which lacks proper control over the supply.

It is very important to demonstrate the generic nature of the proposed mechanism of control over the growth mode. Figure 5.9 is the phase diagram of the In-Sb system with two eutectics like the Ga-Sb system. Following the same logic from GaSb growth results, it should be possible to synthesize antimony tip led InSb nanowires if the system is maintained in region 2 .

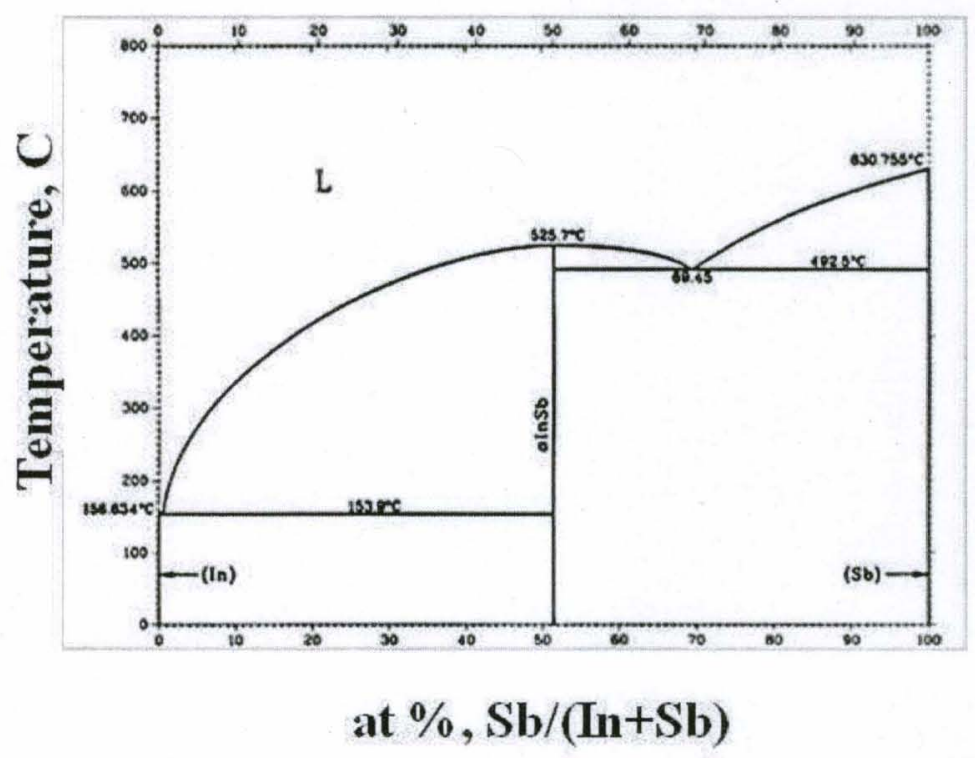

Figure 5.9 Phase diagram of the In-Sb system showing the two eutectics ( $\sim 1 \%$ solubility in indium rich conditions and $32 \%$ solubility in the antimony rich region)

Experimental set up similar to one described in section 3 was set up for the antimony tip led growth of InSb nanowires. The observed growth was analyzed for morphology and composition of the nanowire and the tip. Indeed, a tip led nanowire 
growth was observed. The nanowire had diameter in the 50-70 $\mathrm{nm}$ range and microns in length with tip in the same size range as shown in Fig 5.10.

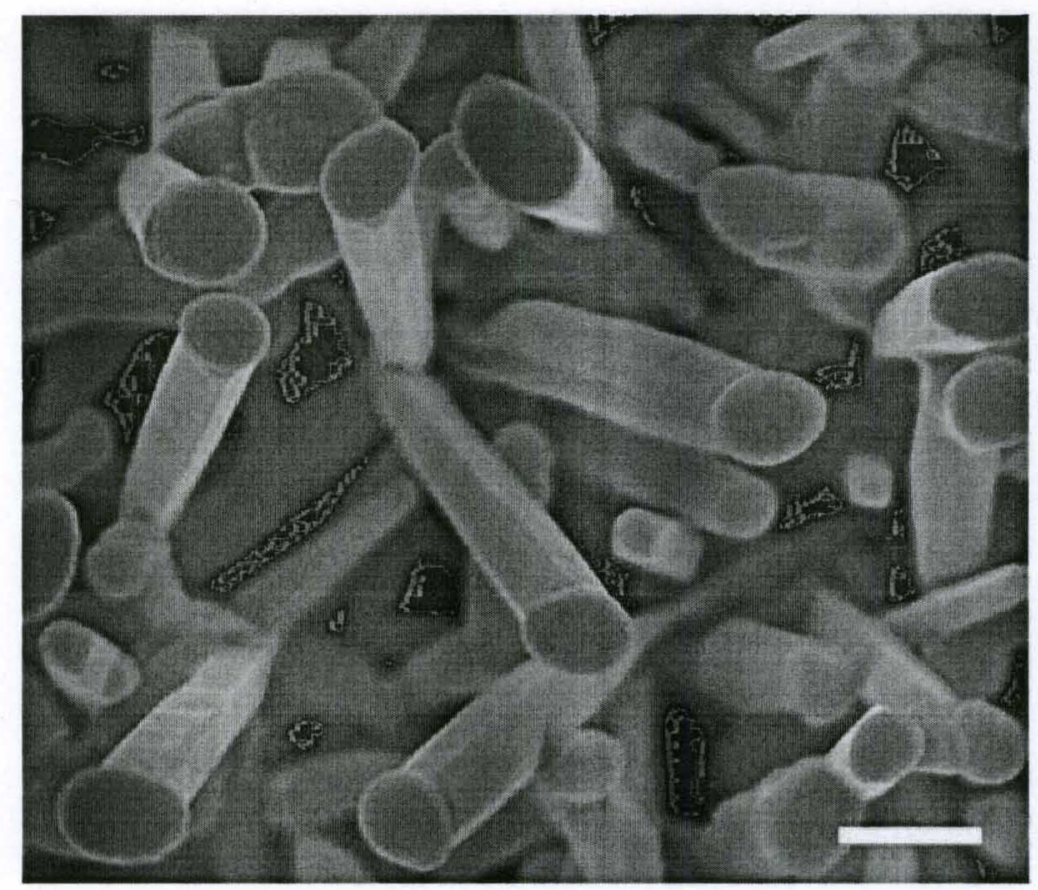

Figure 5.10 SEM micrographs of the as synthesized InSb nanowires where the tip led growth is clearly evident. The scale bar is $100 \mathrm{~nm}$.

SEM EDS elemental map of the wires shown in Fig 5.11 clearly indicates the tip being antimony rich with residual indium as the eutectic is about $32 \%$ indium. In contrast, the gallium tip led GaSb growth did not have any antimony at the tip as the eutectic is extremely small composition. 

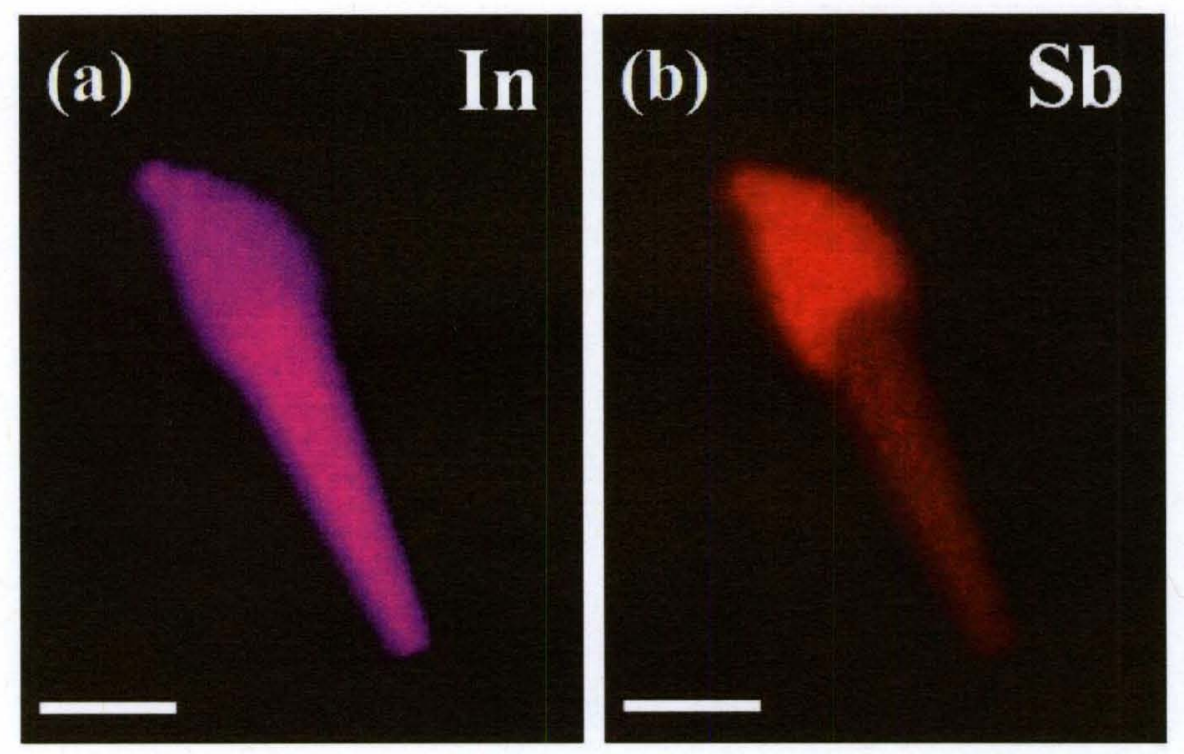

Figure 5.11 SEM EDS elemental map of InSb nanowire showing excess antimony at the tip indicating an antimony tip led growth of the nanowires. The scale bars are $100 \mathrm{~nm}$.

The proposed concept to address the role of equilibrium solubility in controlling nanowire growth mode can also be used to explain the observed tip led growth in $\mathrm{Au}$ assisted silicon nanowire growth as the large solubility would lead to a large critical nuclei size leasing to tip led growth ${ }^{163}$ if the Au droplets are in the 100 s of nm size range. Bulk nucleation has also been observed for nickel cluster assisted silicon nanowires where the large solubility leads to large critical nuclei size but the very large nickel catalyst droplet size leads to bulk nucleation ${ }^{179}$. 


\subsection{Synthesis of GaN nanowires}

\subsection{Self-catalyzed synthesis of GaN nanowires}

GaN nanowires were synthesized at different process conditions to achieve orientation controlled growth. Synthesis was also attempted on different substrates. Earlier work in our group has already shown that growth orientation control can be

obtained by controlling the growth mode $^{173}$ (tip led or bulk nucleation). The synthesis conditions devised for the orientation controlled synthesis are discussed in section 3.2.1.1, where it is the material flux that is the parameter to control the nanowire growth orientation. Another very important difference is the substrate employed in the two approaches. Quartz was used as the substrate when growth mode was the control ${ }^{173}$ where the wetting behavior of gallium droplets was the controlling parameter. Different substrates were used in this study ranging from metals (stainless steel, copper), to semiconductors (silicon and $\mathrm{GaN}$ ) and insulator (quartz). The primary reason is that stainless steel and copper can be used as direct back contact for device applications while the nanowires on quartz would have to be scraped off and deposited onto a conducting substrate. Nanowires grown on $\mathrm{GaN}$ and silicon substrates can be used only when there is a good ohmic contact between the substrate and the connector wires.

Two distinct process conditions were devised to understand the role of material flux and type of substrate on the nanowire growth process. Figure 5.12 is a compilation of the resulting growth obtained on various substrates at each growth condition. One set of process conditions used gallium metal source heated to $750 \mathrm{C}$ at $300 \mathrm{~m}$ Torr with 200 
sccm $\mathrm{NH}_{3}$ flow and other used $\mathrm{GaN}$ powder at $900 \mathrm{C}$ at 20 Torr with $200 \mathrm{sccm} \mathrm{NH}_{3}$ flow. A clear dependence of the nanowire growth as well as orientation of the synthesized nanowires on the substrate and the process parameters was observed. It was observed that experiments using gallium metal at $900 \mathrm{C}$ resulted in the growth of only $\mathrm{GaN}$ crystals on the substrates and no growth was observed on the substrate when using GaN powder at the $750 \mathrm{C}$ conditions.

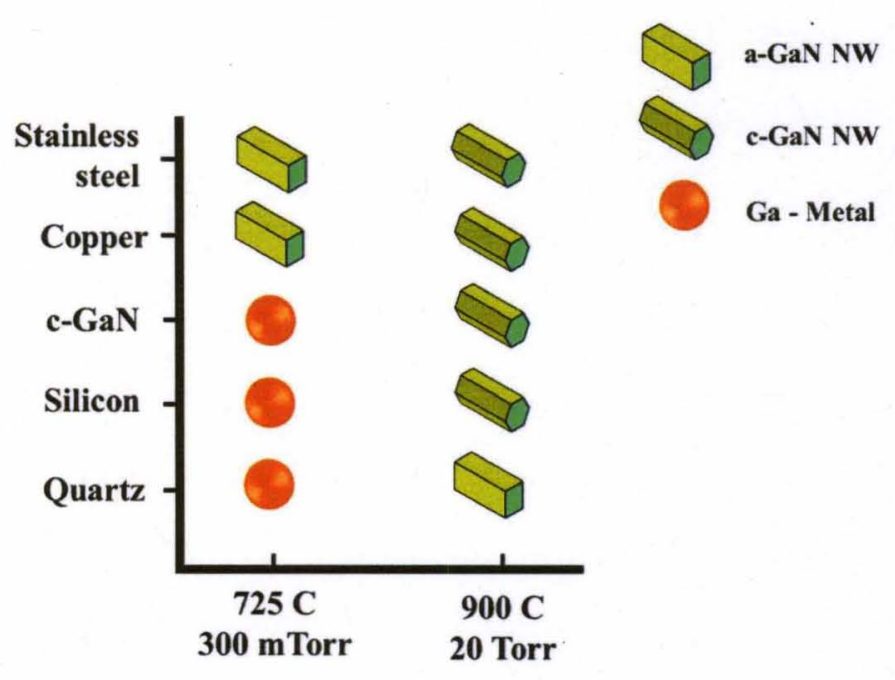

Figure 5.12 Template for process and substrate selection for controlled growth of $\mathrm{GaN}$ nanowires. Metal substrates can always be used to grow nanowires using the developed process window whereas nanowire growth on semiconductor or insulators is heavily affected by the wetting behavior of gallium.

Based on the process conditions and substrate, the nanowire growth and orientation can be controlled. When employing the $750 \mathrm{C}$ conditions, the gallium flux is high from the direct evaporation of the metal and gallium deposition was observed on the non-metallic substrates. This could be due to the fact that at the low substrate temperatures, $\mathrm{GaN}$ reaction might be activated only at the metal surfaces which might 
catalyze the crystal formation to start the nanowire growth. Also, at lower temperatures, the higher order planes might be energetically favorable, leading to the growth of a-plane oriented GaN nanowires.

At the high temperature conditions $(900 \mathrm{C})$, the surface temperatures on all but the quartz substrate would be high enough and the low gallium flux from the GaN powder results in the formation of small crystal nuclei on the surface. Since at very high temperatures, c-plane is the most stable, c-plane oriented nanowires are formed. Gallium vapor preferentially condenses on the preexisting nuclei leading to a tip led growth. However, since quartz is an insulator, the surface temperature would be lower than the other substrates. This although is sufficient to growth GaN crystals, a plane oriented nanowires grow as they are stable at lower temperatures.

As synthesized " $\mathrm{c}$ " plane and "a" plane oriented GaN nanowires synthesized on stainless steel substrates are shown in figure 5.13. This is a clear demonstration of the role of process conditions in controlling the nanowire growth orientation.

(a)

(b)

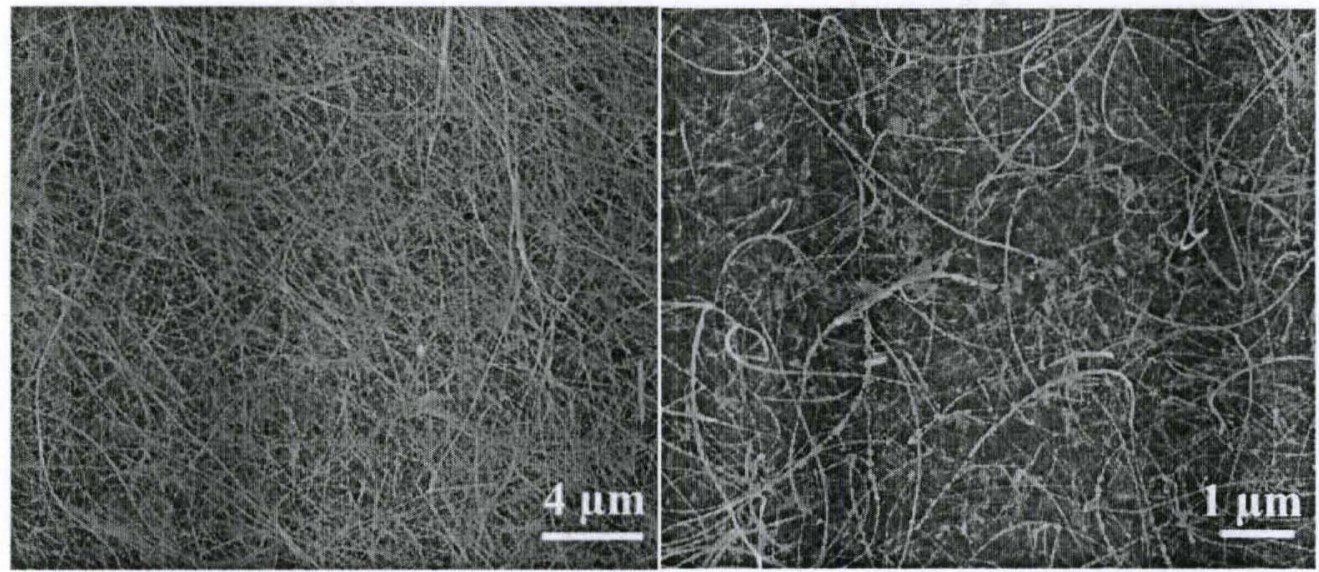

Figure 5.13 SEM micrograph of a) "c" plane and b) "a" plane oriented GaN nanowires synthesized on stainless steel. 
Figure 5.14 shows the as synthesized "a" plane oriented GaN nanowires grown on quartz substrates. The growth is very uniform and the close up image shows the nanowires with $25-50 \mathrm{~nm}$ diameter and 100s of microns length.

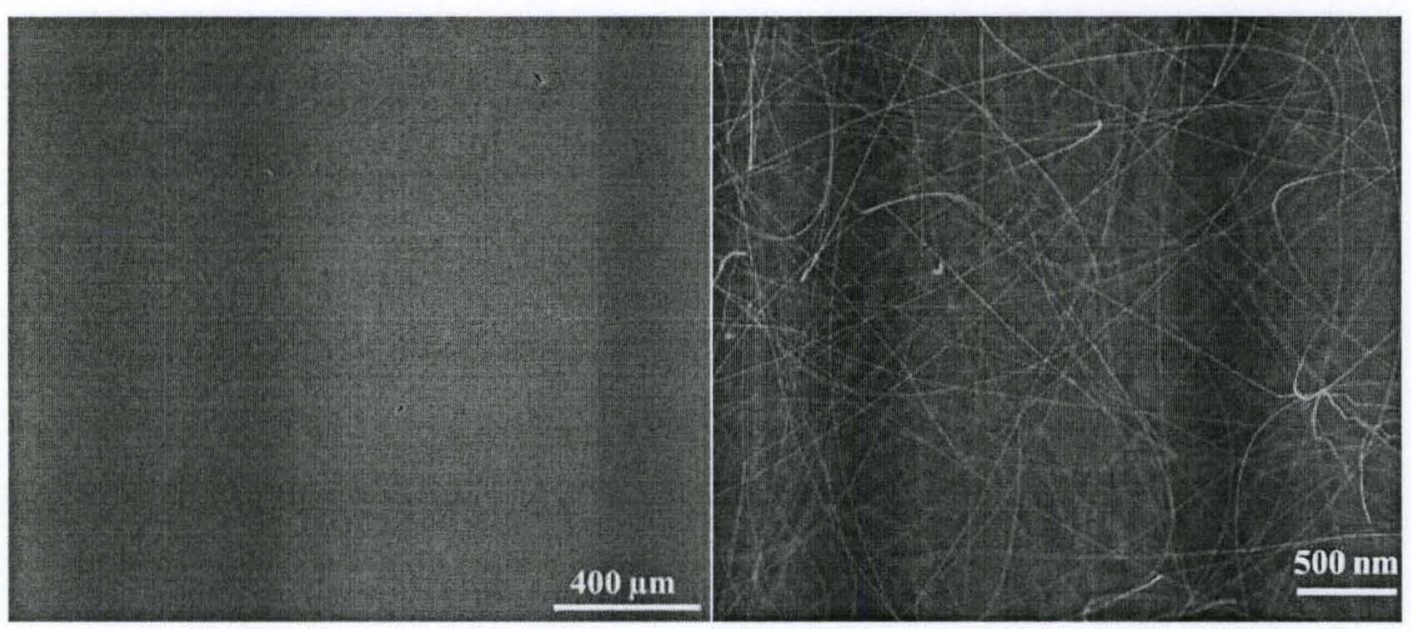

Figure 5.14 SEM micrographs of "a" plane oriented GaN nanowires synthesized on quartz substrates.

Figure 5.15 through 5.17 are the images of the nanowires grown on various substrates. All the nanowires were synthesized at 900C conditions and resulted in "c" plane oriented nanowires.

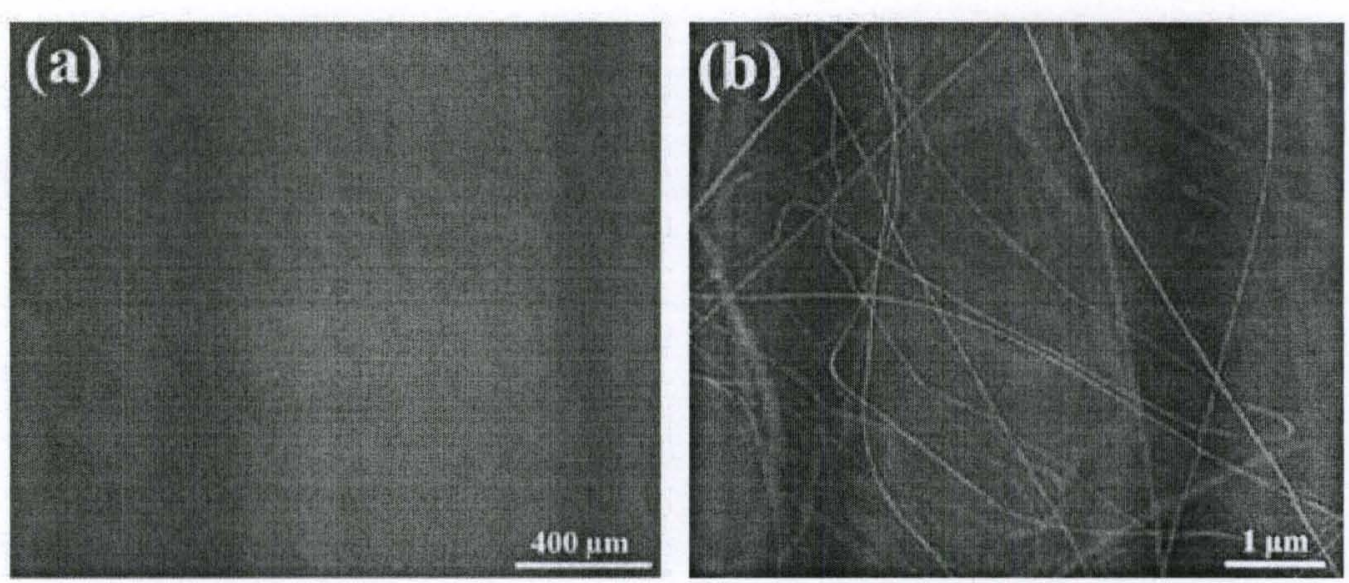

Figure 5.15 SEM micrographs of "c" plane oriented $\mathrm{GaN}$ nanowires grown on copper substrate. 

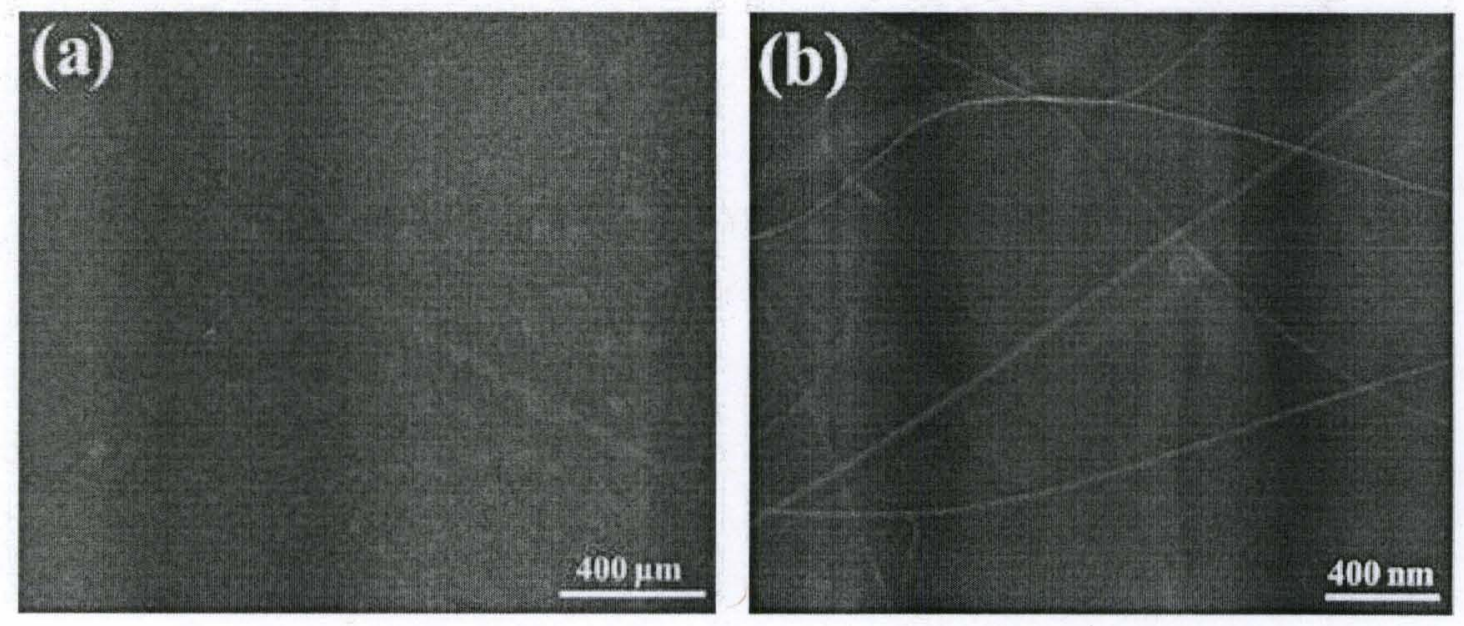

Figure 5.16 SEM micrographs of "c" plane oriented GaN nanowires synthesized on GaN substrate.
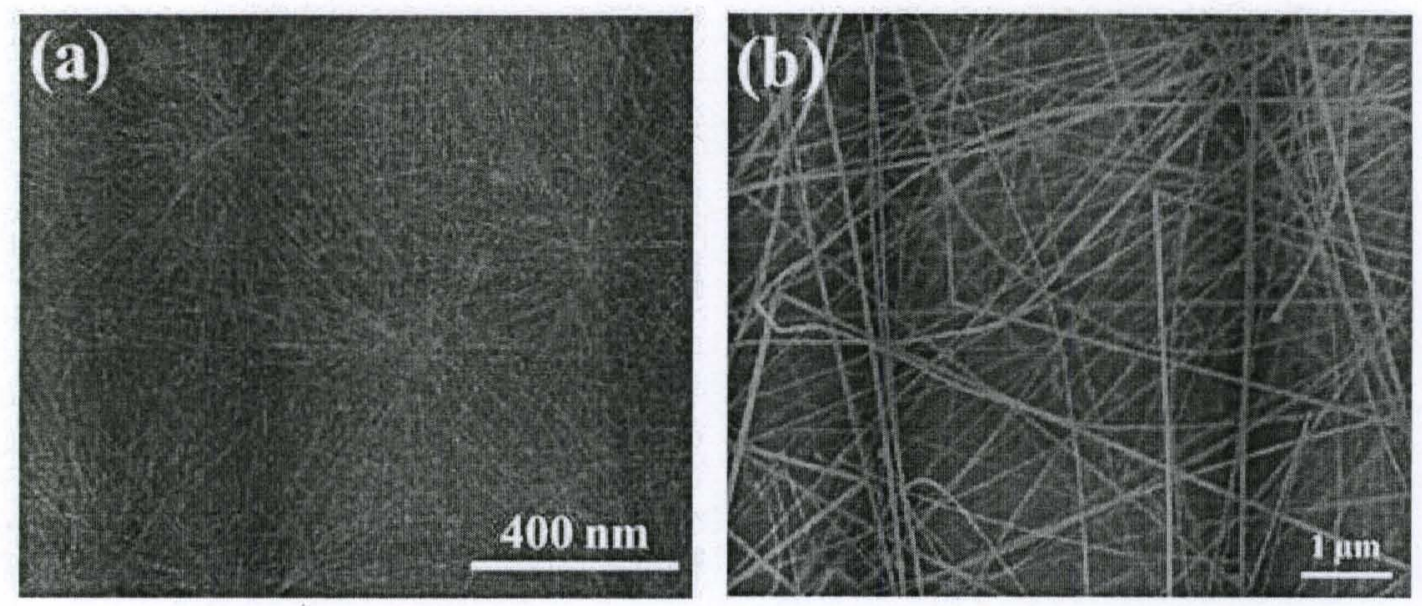

Figure 5.17 SEM micrographs of "c" plane oriented GaN nanowires synthesized on silicon substrate.

The implications of such control are tremendous in device applications where vertical arrays are a preferred mode compared to bulk nucleation. 


\subsubsection{Copper catalyzed synthesis of GaN nanowires}

Synthesis of GaN nanowires without any catalyst led to the growth of long nanowires in a mat like morphology. Synthesis of vertical arrays of nanowires is

challenging by this method as there is not precise control over the density and spacing of the nanowires. Using a catalyst can help to direct the growth of nanowires. Various catalysts such as gold, nickel etc have been used for the synthesis of $\mathrm{GaN}$ nanowires and it has been shown that they can create trap states in the material causing deterioration of the material properties.

Copper nanoparticles (diameter $<100 \mathrm{~nm}$ ) were used as catalyst for the synthesis of $\mathrm{GaN}$ nanowires. Under similar conditions described for the synthesis of $\mathrm{c}-\mathrm{GaN}$ nanowires i.e. $900 \mathrm{C}$ with $200 \mathrm{sccm} \mathrm{NH} \mathrm{NH}_{3}$ at 20 Torr the bare substrate was replaced with the ones coated with copper nanoparticles.

Tip led growth of GaN nanowires with three distinct morphologies was observed. In one case, the nanowires were coiled with 10 s of microns long with diameters in the 20$50 \mathrm{~nm}$ range as shown in figure 5.18. The coiled growth could be due to a constant instability of the droplet on the nanowire surface. Further characterization on the droplet/nanowire interface is needed to study the phenomenon. 

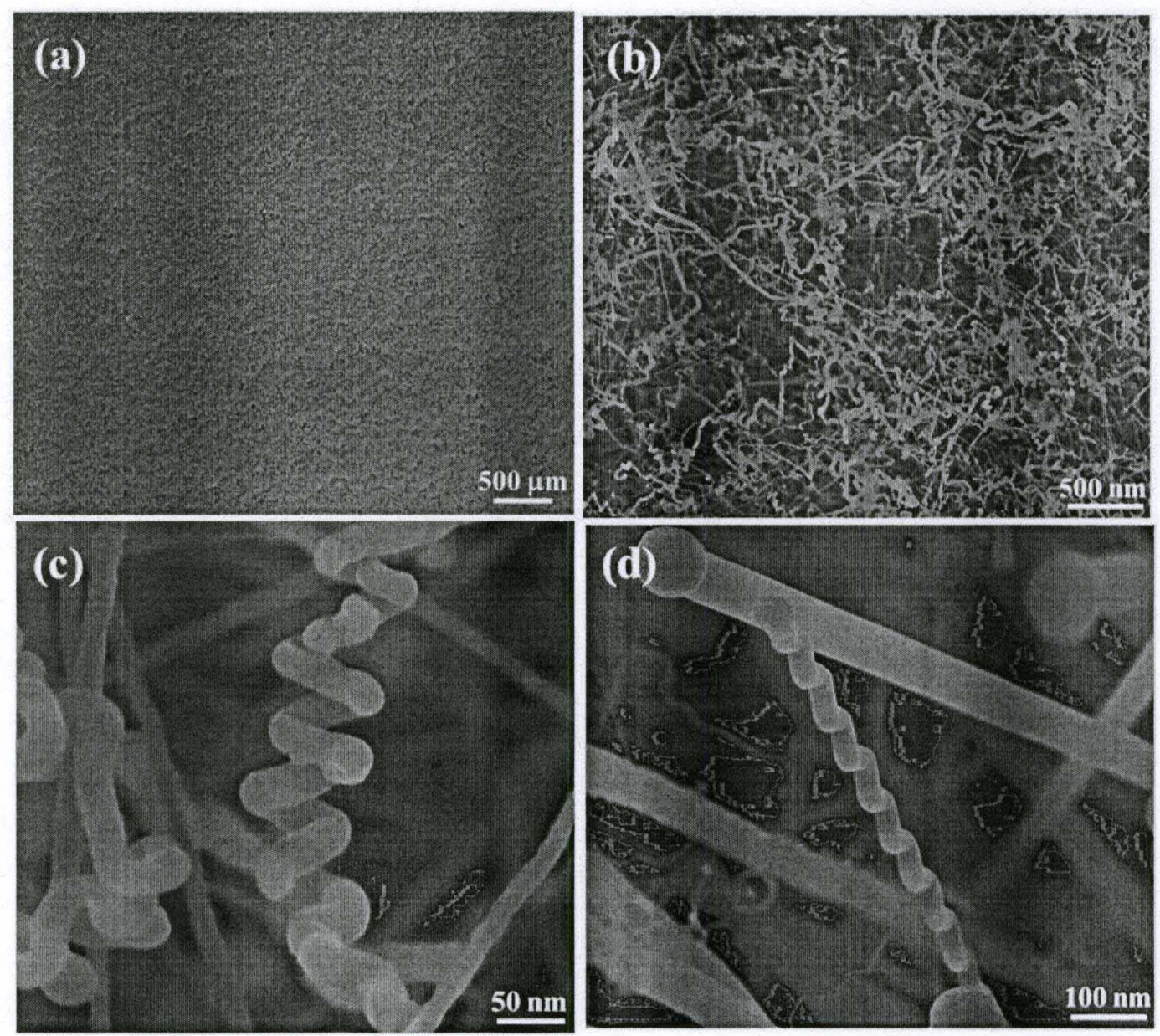

Figure 5.18 SEM images of the coiled GaN nanowire morphology of GaN grown using copper tips. The nanowires are $10 \mathrm{~s}$ of microns in length and $10-50 \mathrm{~nm}$ in diameter. The coil could be the result of constant copper droplet instability on the growing nanowire.

Straight GaN nanowires with copper tips were also observed. The nanowires were 2-5 $\mu \mathrm{m}$ in length and $\sim 100 \mathrm{~nm}$ in diameter. Faceting of the nanowire and the copper droplet at the tip is also evident. This is the kind of growth needed to get to vertical arrays. 


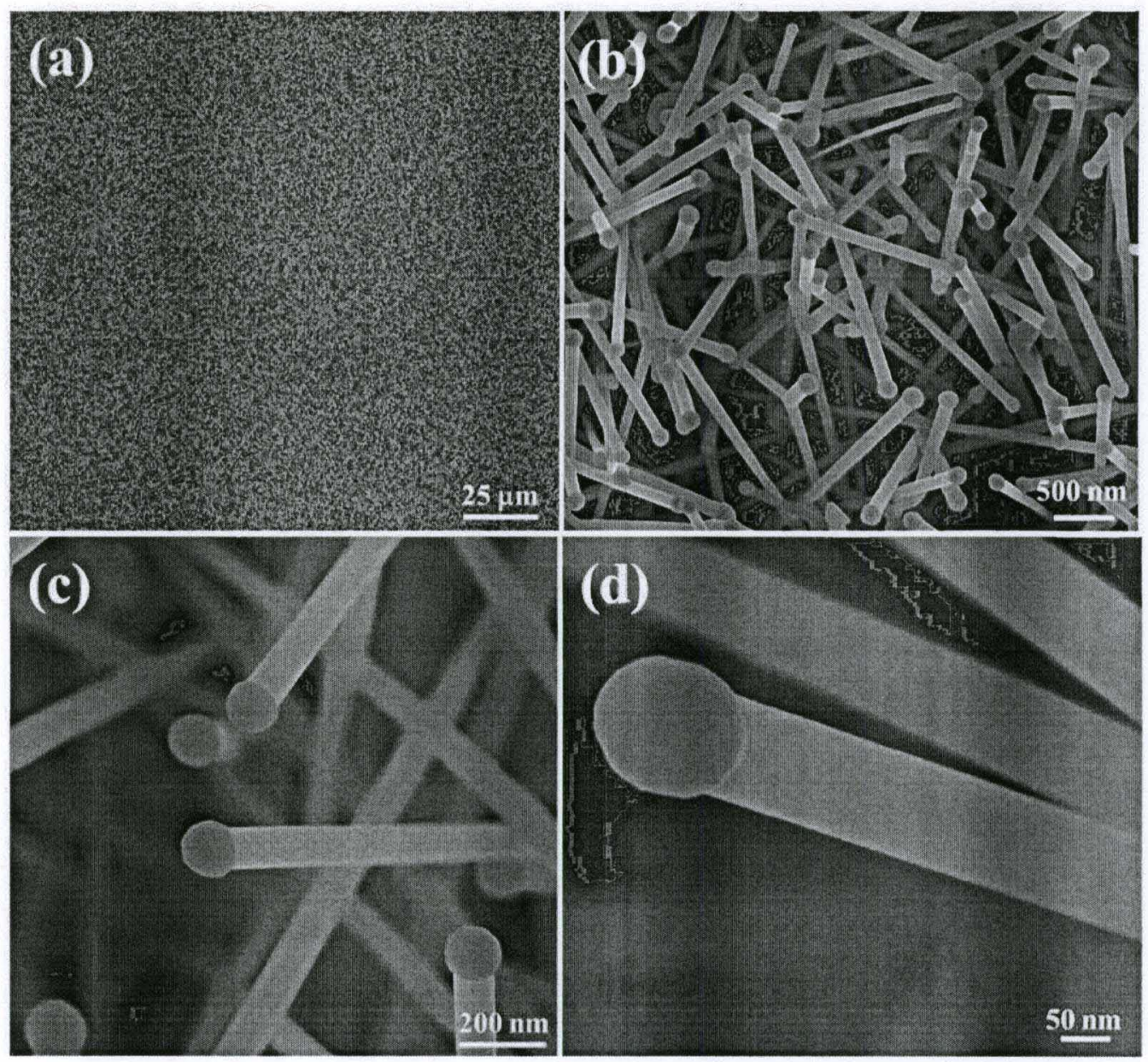

Figure 5.19 SEM images of the copper catalyzed GaN nanowires showing the straight nanowire growth suitable for vertical array format. The nanowires are $\sim 100 \mathrm{~nm}$ in diameter and 2-5 $\mu \mathrm{m}$ in length. Faceting of the tip is clearly evident.

The most interesting morphology however, shown in figures 5.20. The nanowires grow as branches on the straight nanowires. The branched nanowires are single crystalline, have a copper tip as well but bend $120^{\circ}$ periodically. HRTEM images of the nanowires shown in figure 5.21, show that the nanowires are single crystalline and 
orientation of the nanowires is maintained by the six fold symmetry of GaN. The bend does not have any kinks or dislocations. This could be caused by the instability of the copper droplet on the nanowire tip and the mechanism needs further investigation.
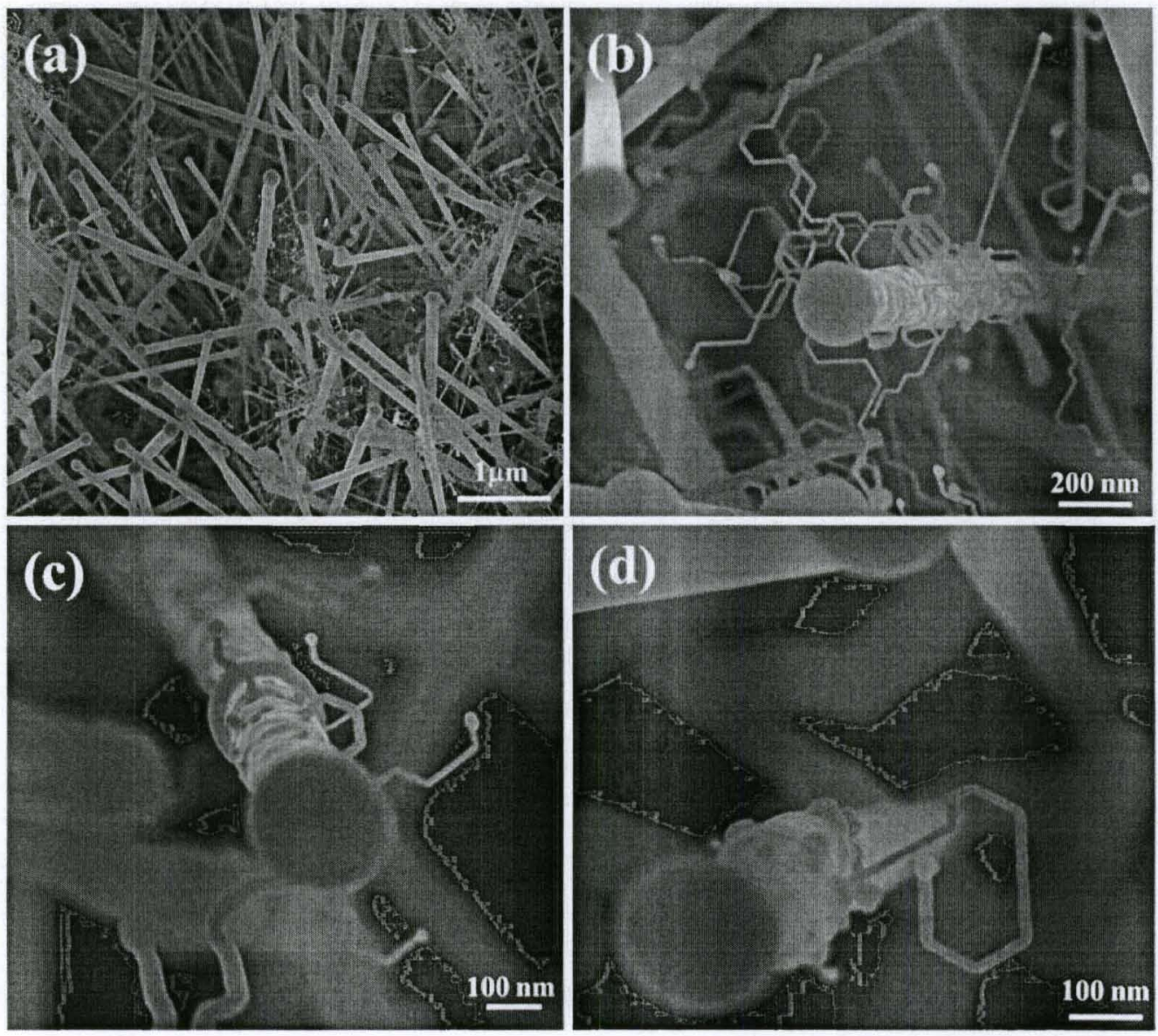

Figure 5.20 SEM images of the branched growth on straight GaN nanowires. The branches are also copper tip led with diameters in the 10-20 nm range. The branches are straight to some length and then bend $120^{\circ}$ possibly due to droplet instability. 

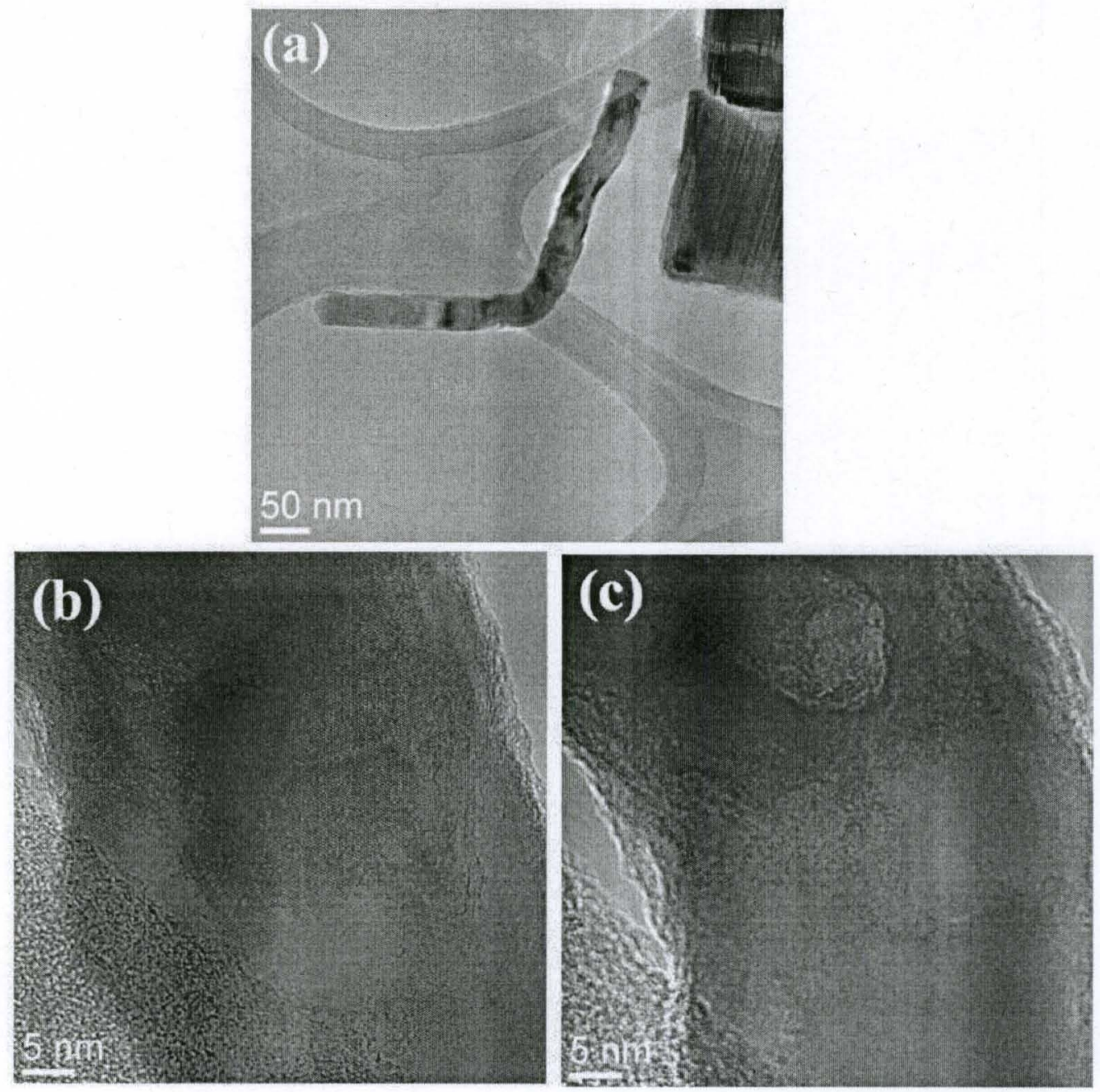

Figure 5.21 HRTEM images of the $120^{\circ}$ bent GaN nanowire branch. The arm as well as the bend does not have any stacking faults/kinks. The orientation of the nanowire is preserved as $\mathrm{GaN}$ has a six fold symmetry which also could be the reason for the observed bend angle of the nanowire. 
CHAPTER 6

\section{SYNTHESIS OF InGaN ON GaN NANOWIRES}

\subsection{Introduction}

This chapter includes the results from the observed morphology of InGaN layers growth on GaN nanowire substrates. Characterization of the InGaN films in bulk and nanoscale for crystallinity, composition and composition uniformity is included. Analysis of the observed dependence of the heteroepitaxial growth on the orientation of the nanowire substrate is also discussed. Preliminary results on the photoactivity measurements on the bare $\mathrm{GaN}$ nanowires and $\mathrm{InGaN} / \mathrm{GaN}$ structures are also presented.

\subsection{InGaN growth on GaN nanowire substrates}

The synthesized GaN nanowires are now used as substrates for the synthesis of InGaN alloys with controlled composition. Since a very precise control over the precursor flux can be achieved using gas phase supply, MOCVD was employed. This section includes a discussion on the observed growth, results from the composition, 
crystallinity and band gap measurements along with some interesting growth and processing issues.

\subsubsection{Morphology}

The main idea of this work is that nanowires can act as strain relaxing substrates and help in mitigating phase segregation. This will be proved only by the growth of single crystalline InGaN with controlled composition on GaN nanowires. Also, the ability to synthesize GaN nanowires of controlled orientation allowed for the study of any orientation dependence of InGaN growth on GaN nanowire substrates.

Figure 6.1 is the compilation of $\mathrm{InGaN}$ growth on $\mathrm{GaN}$ nanowires. Growth evolution of InGaN on "c" plane and "a" plane GaN nanowires is shown in the figures ac and d-f respectively. Since it was possible to synthesize GaN nanowires with controlled orientation on stainless steel, comparative studies of orientation dependence would be free of any substrate led variations. 

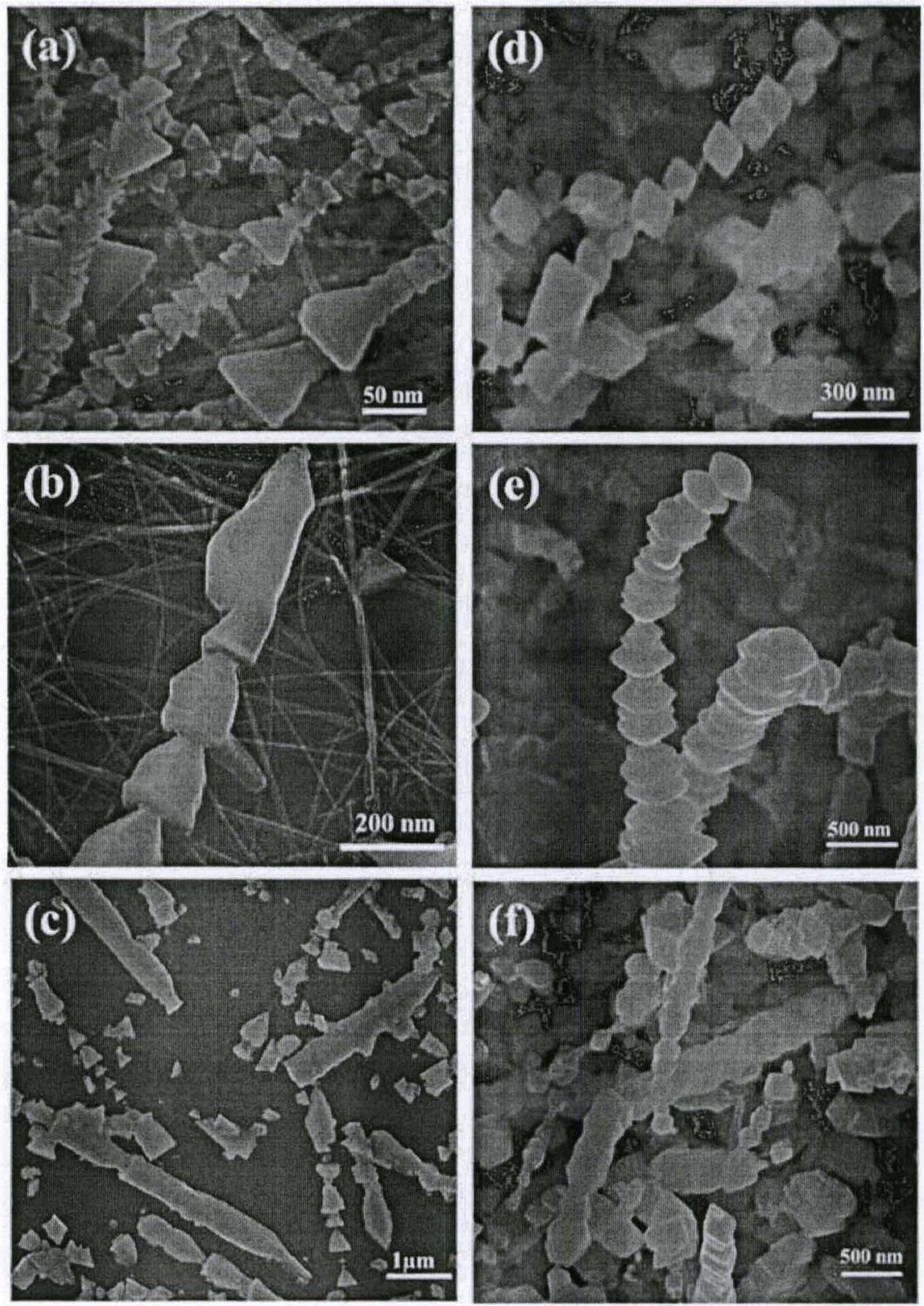

Figure 6.1 SEM micrograph of the evolution of $\mathrm{InGaN}$ growth a)-c) c-GaN and d)-f) $\mathrm{a}-\mathrm{GaN}$ nanowires. Island growth, coalescence followed by a shell formation is the general observation. The hexagonal islands grow along the length of the c-GaN nanowires while growth is perpendicular to the length in the case of a-GaN nanowires. 
Composition controlled synthesis of InGaN alloys on $\mathrm{GaN}$ nanowires was attempted via control of the various process parameters such as precursor flux, substrate temperature etc. Details of the process window developed will be discussed later in the chapter. The synthesis process follows a feedback system where after one synthesis experiment the material is extensively characterized to understand the growth. Single parameter variation is pursued deriving from the previous result to attain the desired composition and crystallinity.

The evolution of the growth of InGaN layers grown on c-GaN and a-GaN nanowires is shown in figures 6.1 a)-c) and d)-f) respectively. The common feature of the growth is the hexagonal island formation, coalescence and finally the formation of a epitaxial shell on the nanowires. The orientation dependence of the heteroepitaxial growth on the nanowires is reflected in the orientation of the islands and the nature of the shell formation. A thorough investigation into the mechanism of the shell formation will be presented later in the section. This result shows that crystalline growth can be obtained on the nanowires. The formation of crystalline islands coalescing into a uniform shell on the nanowires is the main result for this section.

\subsubsection{Crystallinity from XRD}

After the growth is analyzed for morphology confirming it is a crystalline looking growth. The most important step is to check for the composition of the synthesized alloy which has to be measured and corroborated using a number of techniques for a consistent and accurate measurement. From a synthesis point of view, it is important to find out the 
nature of growth. Irrespective of the composition, if the InGaN layer is not a single crystal, it is not really a useful result as the grain boundaries will act as trap sites for the charge carriers destroying any electronic charge transport properties. Hence, the samples were analyzed in XRD for crystallinity. The XRD peak as well as the variation in the lattice parameter indicative of composition of the InGaN alloy is shown in figure 6.2. Here, XRD is used as a bulk characterization tool to investigate the crystallinity of the synthesized alloy.

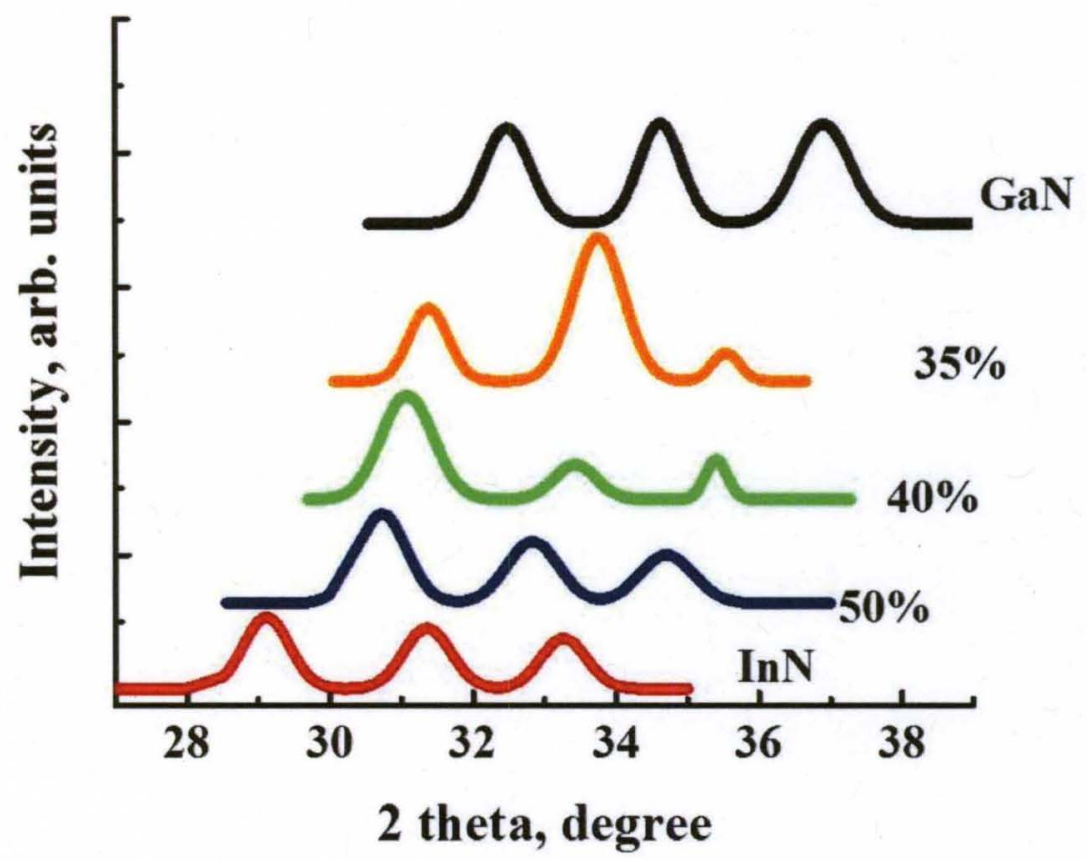

Figure 6.2 Compilation of the XRD peaks obtained from the synthesized InGaN alloys. XRD peaks from $\mathrm{GaN}$ and $\mathrm{InN}$ are included to show that the peaks lie within the two composition extremities.

Presence of peaks is indicative of the crystallinity of the material. Also, since the peaks are a direct measure of lattice parameters, spectra with shifted peaks are indicative 
of a different lattice parameter i.e. composition. The InGaN peaks lie between the GaN and InN peaks which should be the case and confirms that the material is InGaN. Following Vegard's law ${ }^{180}$ the composition of the InGaN layers is determined using the corresponding $\mathrm{GaN}$ and InN peaks. The presence of a single set of peaks is a clear indication that only a single phase is present. If there was phase segregation into a gallium or indium rich phases, two or more (multiple) peaks would show up on the XRD spectra. Hence, this is a demonstration of the proposed concept that nanowires do in fact act as strain relaxing substrates as the highest strain is caused at the mid compositions and the XRD data on the mid composition InGaN shows no phase segregation.

\subsubsection{Band gap from diffuse reflectance}

Continuing with the theme of bulk characterization tools, optical measurements were performed to check for the band gap of the synthesized InGaN alloys. InGaN alloys should have band gaps between $3.4 \mathrm{eV}(\mathrm{GaN})$ and $0.8 \mathrm{eV}(\mathrm{InN})$. This would be another indication of the compositions synthesized. However, standard transmission or absorbance measurements were not possible for our samples due to the stainless steel substrate. Diffuse reflectance measurements were performed to check for the band gap. The reflectance signal (R) has to be converted into usable numbers that can be used to deduce the band gap of the alloys ${ }^{159}$. Analogous to the Tauc plot of $(\alpha h v)^{2} v s(h v)$ used to determine the direct band gap of materials, the new conversion parameter from the

diffuse reflectance signal (R) is used. A plot of $\quad\left[\ln \left(\frac{R_{\max }-R_{\min }}{R-R_{\min }}\right)\right]^{2}$ vs Energy is used to determine the direct band gap of the material ${ }^{159}$. Figure 6.3 is the compilation of the band 
gap measured from the diffuse reflectance from various InGaN compositions synthesized. The straight lines from the spectra indicate a direct band gap which is very important for the material to be a good absorber and generate electron hole pairs efficiently.

The range of band gaps synthesized from $0.7 \mathrm{eV}$ to $3.4 \mathrm{eV}$ and all the compositions indicate that nanowires can be used as strain relaxed substrate for all compositions. However, the region of interest is in the $40-60 \%$ indium content where the band gap is $\sim 2 \mathrm{eV}$.

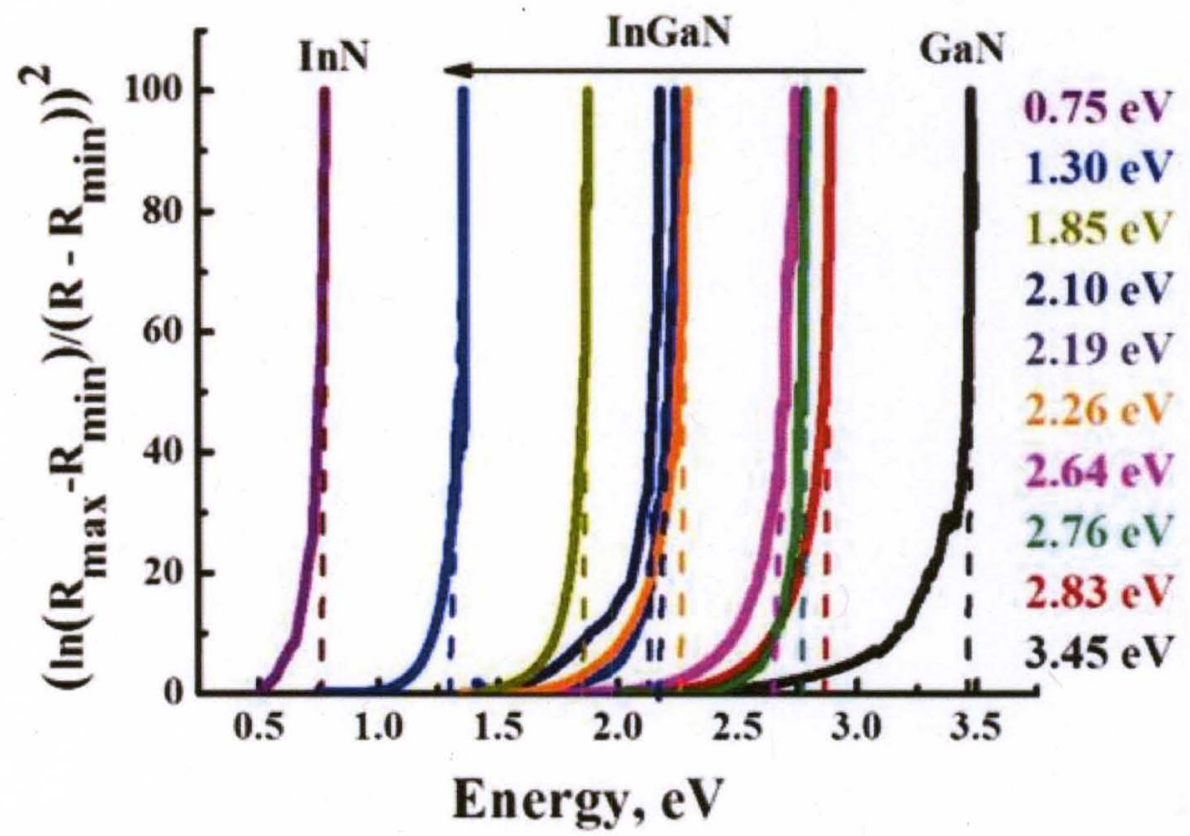

Figure 6.3 Compilation of all the band gaps measured on the InGaN samples measured via diffuse reflectance the band gaps of all the $\mathrm{InGaN}$ alloy compositions from $0.7 \mathrm{eV}$ to $3.4 \mathrm{eV}$. 


\subsubsection{Epitaxy and composition from electron diffraction}

Having ascertained the crystallinity and bulk composition of the alloys, now it is important to determine the crystal quality and material distribution in the nanoscale since bulk measurements can sometimes average out the nanoscale localized composition modulation. Epitaxial relation to the underlying substrate also has to be understood to determine the growth mechanism. HRTEM was employed to study the crystallinity and epitaxy of the $\mathrm{InGaN}$ growth on GaN nanowires. Figure 6.4 is a representative HRTEM image of InGaN layer along with the GaN nanowire for an alloy with $90 \%$ indium content (almost the maximum lattice separation from $\mathrm{GaN}$ ). This sample is very useful to study the heteroepitaxial growth behavior on nanowires as the stress related effects will be at the maximum amplitude due to the almost maximum variation in the lattice parameter of the growth species and the substrate. 


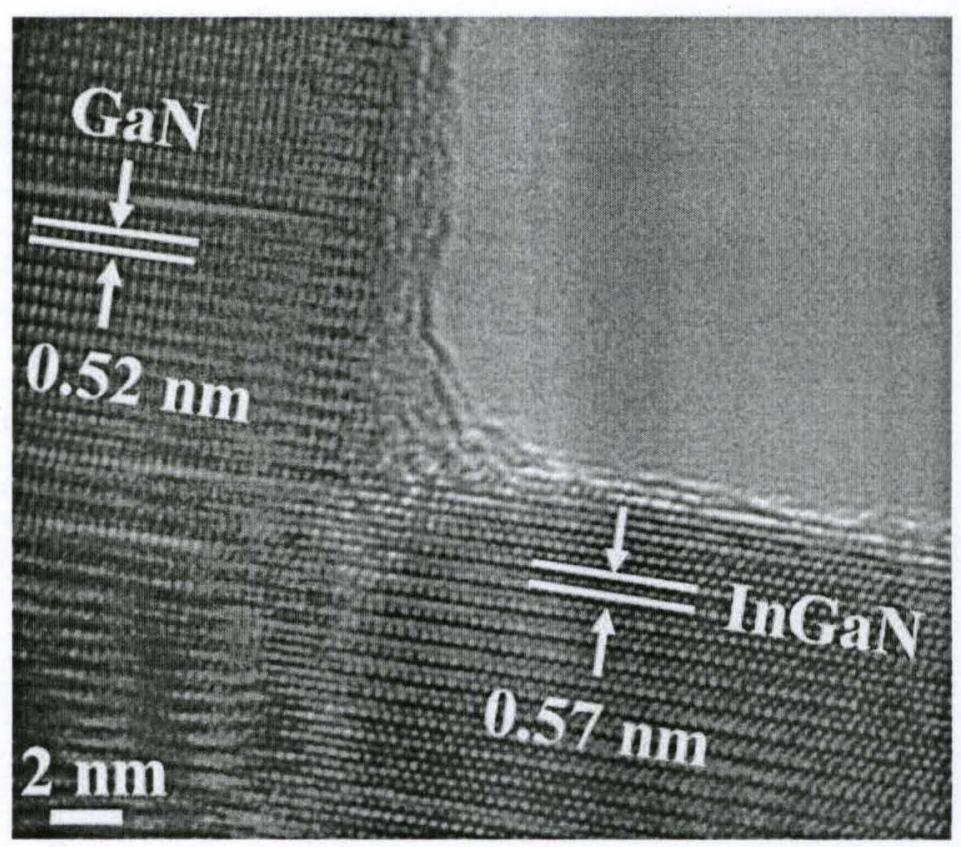

Figure 6.4 Representative HRTEM image of the InGaN growth on GaN nanowire. InGaN layer has a larger lattice constant that the GaN nanowire as indicated in the figure.

As shown in the figure 6.5 , the InGaN layer is single crystalline with few dislocations that is a reflection on the strain relaxed heteroepitaxial growth. No new defects or misfit dislocations are created. Epitaxy leads to the translation of the substrate orientation to the growing layers and that is the reason for the observed difference in the arrangement of the hexagonal islands on the $\mathrm{c}-\mathrm{GaN}$ and a-GaN nanowires. Details of this process will be discussed later in the chapter.

Localized study of the crystallinity and epitaxy can be conducted using electron diffraction analogous to XRD (bulk). Figures 6.5a and b show two representative SAED spot patterns obtained from InGaN/GaN structures with different InGaN compositions. 
(a)

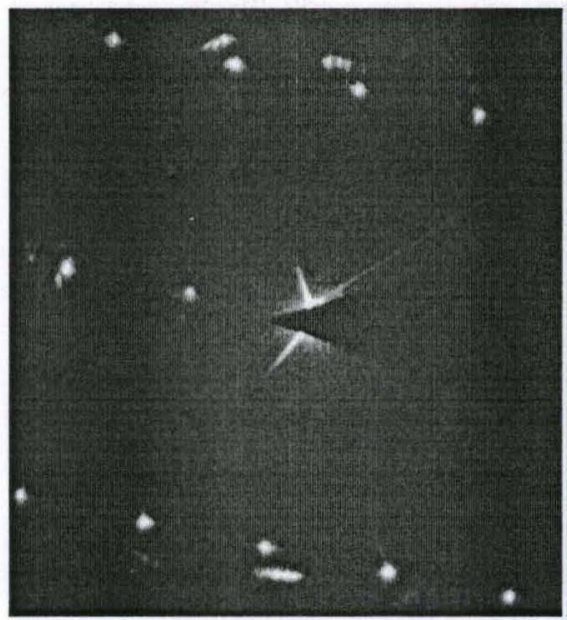

$\operatorname{In}_{0.9} \mathrm{Ga}_{0.1} \mathrm{~N}$ (b)

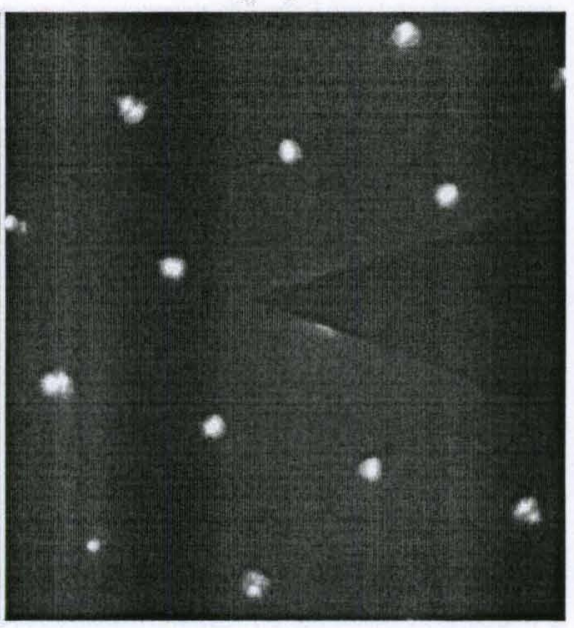

$\mathrm{In}_{0.5} \mathrm{Ga}_{0.5} \mathrm{~N}$

Figure 6.5 Sample SAED spot patterns obtained from $\mathrm{InGaN}$ layers on $\mathrm{GaN}$ nanowires. The difference in the patterns is the relative separation of the spots which is indicative of heteroepitaxial structures with different lattice parameters. Presence of only two identical symmetric sets of spot patterns shows single crystalline growth.

One set of spots is from GaN while the other is from InGaN layer. The distance of the spots from the center of the pattern is a measure of the lattice spacing of the material. In figure $6.5 \mathrm{a}$ and $\mathrm{b}$ there are two symmetric sets of spots with different separation distance from the center. This indicates two single crystalline specimens with different lattice parameters but same crystal structure are present (SINGLE CRYSTALLINE HETEROEPITAXIAL GROWTH). The absence of any other or stray peaks marks the absence of any other phases that means NO PHASE SEGREGATION. There is no POLYCRYSTALLINE GROWTH also which would lead to a ring like pattern. The 
distance from the center is inversely proportional to the lattice parameter. This means any InGaN layer on $\mathrm{GaN}$ will have spots closer to the center and the $\mathrm{GaN}$ lattice spots will be the ones farther from the center. So, for the InGaN system, GaN will have the spots farthest from the center while InN with the largest lattice spacing will have the spots closest to the center. Since GaN peaks are always present in the analysis, they can be used as internal calibration for all the lattice spacing measurements. The intensity profiles of the spot patterns can be used to measure the distances, hence the lattice parameters.

A representative intensity profile measured from an SAED spot pattern in shown in figure 6.6. The intensity profile of the set of spots chosen by the rectangle shown in the SAED pattern is presented. The is used to estimate the composition using Vegard's law $^{180}$.

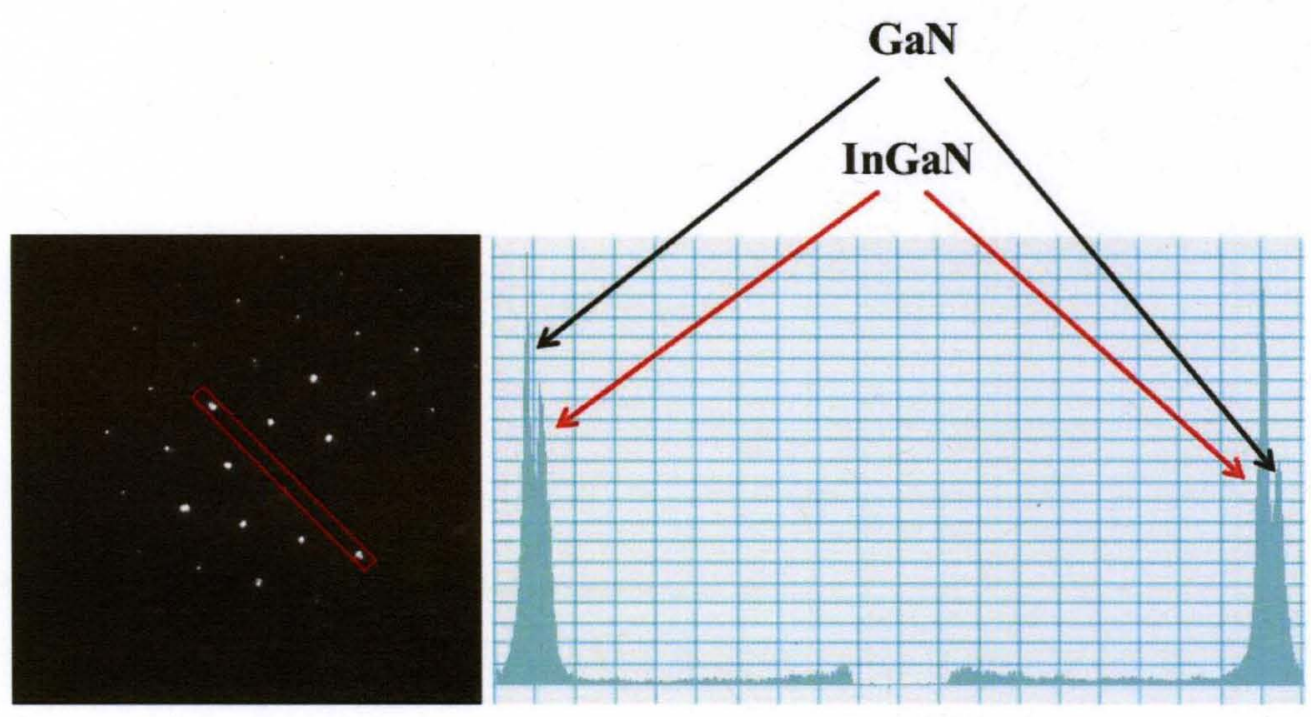

Figure 6.6 Representative SAED pattern with an intensity profile showing the two sets of peaks. Peaks closer to the center are $\mathrm{InGaN}$ and $\mathrm{GaN}$ peaks are farther from the center. Spots chosen are within the red rectangle in the SAED pattern. 
Similar intensity profiles are measured for different InGaN compositions synthesized and used to estimate all the synthesized compositions. Figure 6.7 shows the compiled intensity profiles. All the profiles are normalized wrt the GaN separation wherein the indium content can be measured by the ratio of the separations.

Each normalized InGaN peak position is used to estimate the indium content weighed against the maximum separation of $\mathrm{InN}$ from $\mathrm{GaN}$. Also, elemental composition is measured using TEM EDS spot measurement and is included in the peak label. The two numbers match within measurement error limits.

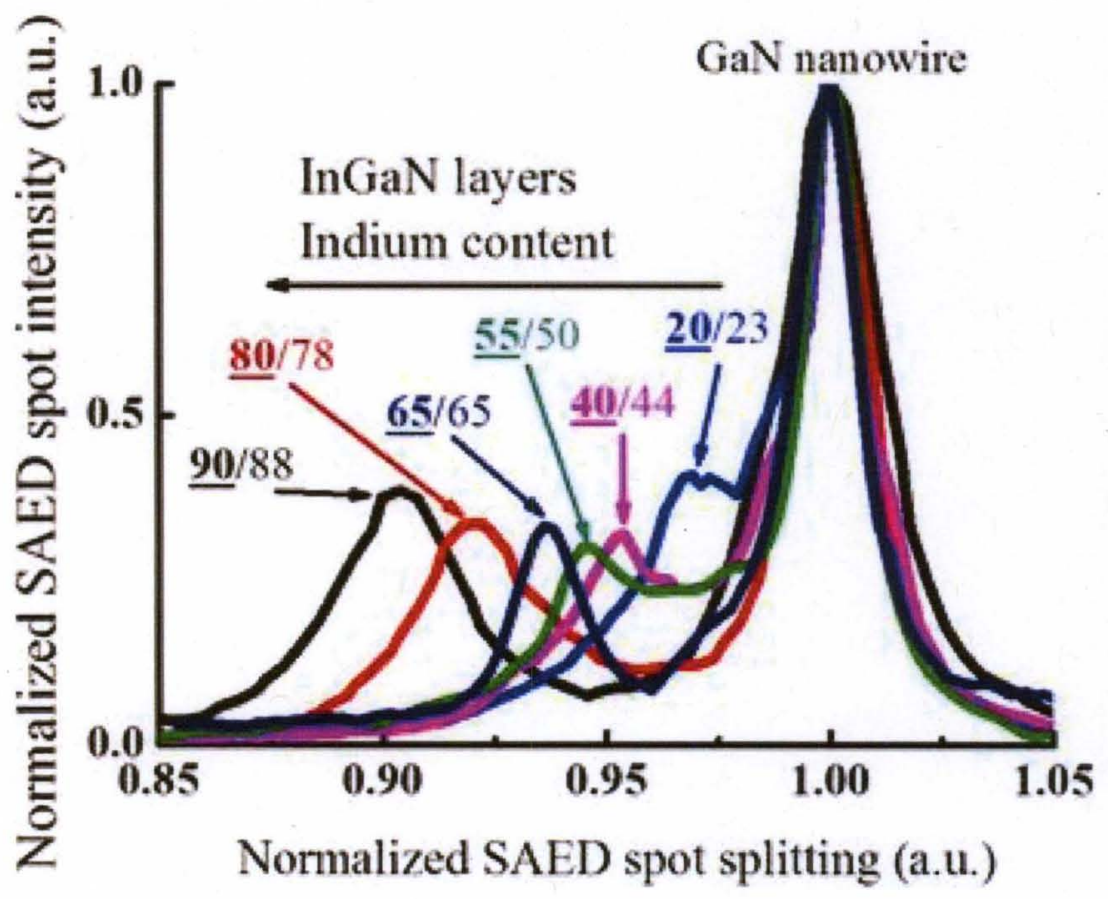

Figure 6.7 Compilation of the intensity profiles from the SAED patterns. All the measurements are normalized wrt GaN peak. The increased spacing of the InGaN peaks from the GaN peak is a measure of the increasing shift in lattice constant translating to increasing indium content InGaN alloys. We have synthesized InGaN alloys from InN all 
the way to GaN. Each InGaN peak is labeled with two numbers. The first is the estimated Indium content measured from the peak positions and the second number is the composition measured from TEM EDS.

\subsubsection{Uniformity of Indium/Gallium distribution in InGaN growth}

Once the compositions are analyzed, the focus is to determine the uniformity of composition (distribution of the indium and gallium species) to ascertain the presence of phase segregation. Uniformity of material distribution was imaged via EDS. EDS is a useful to determine identify and quantify the individual elements in a sample. This can be measured in an SEM (macro scale over a lot of nanowires) as well as in a TEM where a localized measurement is possible. EDS mapping in an SEM was not employed as the resolution is not high enough and for the spot analysis underlying $\mathrm{GaN}$ nanowire adds to the gallium signal which will always underestimate the indium content. Hence, TEM EDS was employed. Figures 6.9 and 6.10 shows EDS elemental maps of InGaN growth on "c" plane and "a" plane oriented GaN nanowires respectively.

In both the images, the GaN nanowire shows up in the gallium map as a thin line but is missing from the indium map and shows up as region of no growth. 


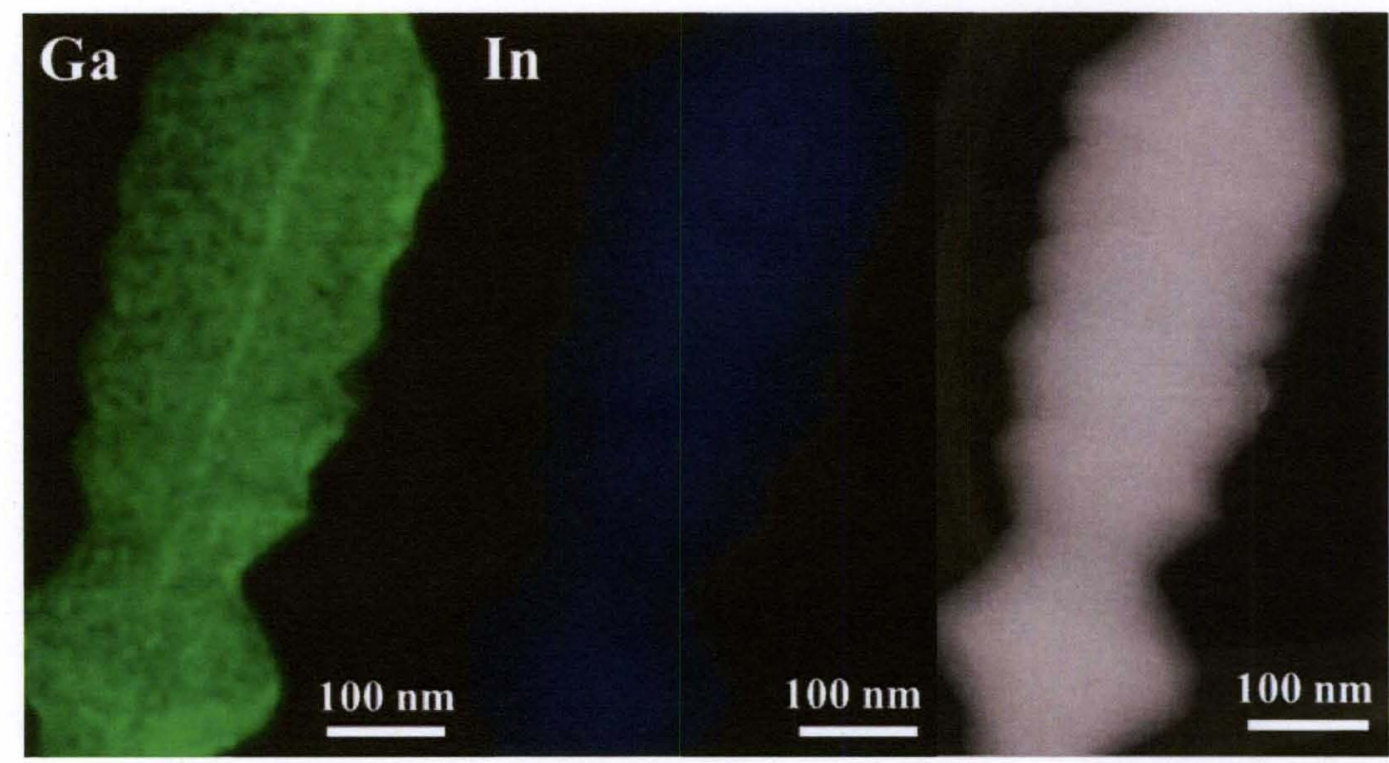

Figure 6.8 TEM EDS elemental map of InGaN growth on "c" plane oriented GaN nanowire. The nanowire is clearly visible in the gallium map and leaves a conspicuously blank pattern in the indium map. The uniform distribution of gallium and indium is clear in the maps. An image of the $\mathrm{InGaN}$ islands on $\mathrm{GaN}$ nanowire is also included for comparison. 


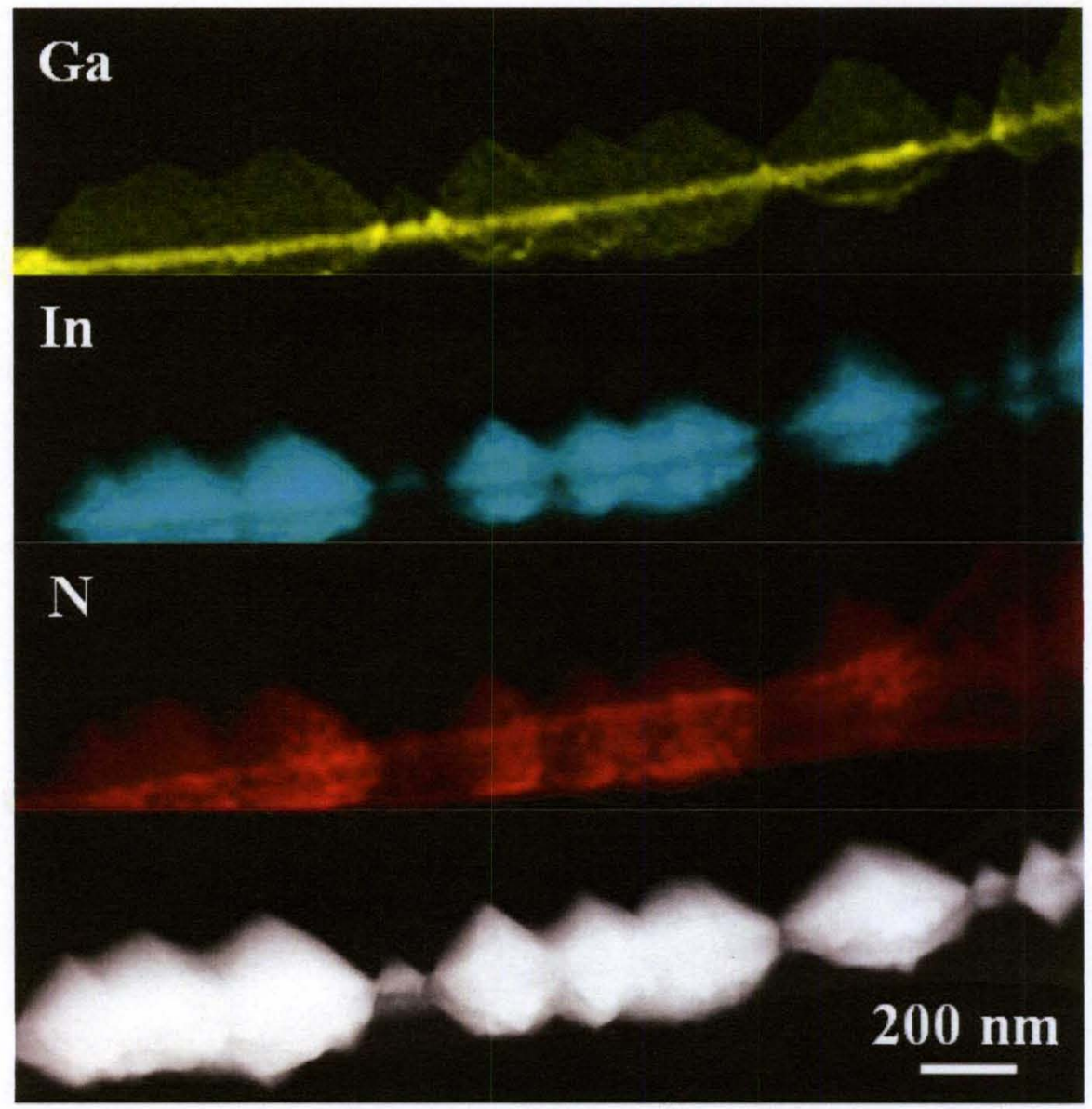

Figure 6.9 TEM EDS elemental map of InGaN growth on "a" plane oriented GaN nanowire. The nanowire is clearly visible in the gallium map and leaves a conspicuously blank pattern in the indium map. The uniform distribution of gallium and indium is clear in the maps. An image of the InGaN islands on $\mathrm{GaN}$ nanowire is also included for comparison.

In the elemental maps, the material distribution is uniform within the measurement limits of the TEM EDS mapping which is in the nm scale. 
As discussed earlier, SAED collects signals from 10 s of $\mathrm{nm}$ large areas. To determine if the material segregation is in the nanoscale, CBED measurements were performed. CBED patterns collected from $<5 \mathrm{~nm}$ spots which would be sufficient to detect composition segregation in the nanoscale. The discs obtained from CBED measurements can be used to measure the lattice spacing analogous to the spots in SAED. Figure 6.10 shows the lattice spacing measurements at the same [010] zone axis for all samples. The measurements are in pixels as $\mathrm{GaN}$ and $\mathrm{InN}$ lattice parameters are also included in pixels and ratios were used to estimate the composition. Measurements were made on multiple nanowires from one sample and repeated for samples with varying indium content.

It is clear from the figure that although there were a few variations in compositions between wires from one sample, the composition numbers were consistent. This was true for different samples with different InGaN compositions as well as shown in figure 6.10. The variation in the composition for different nanowires from one sample is either due to non-uniform precursor distribution in the chamber that can be addressed by reaction tuning or due to the nature of the $\mathrm{GaN}$ nanowires grown on stainless steel substrates. 


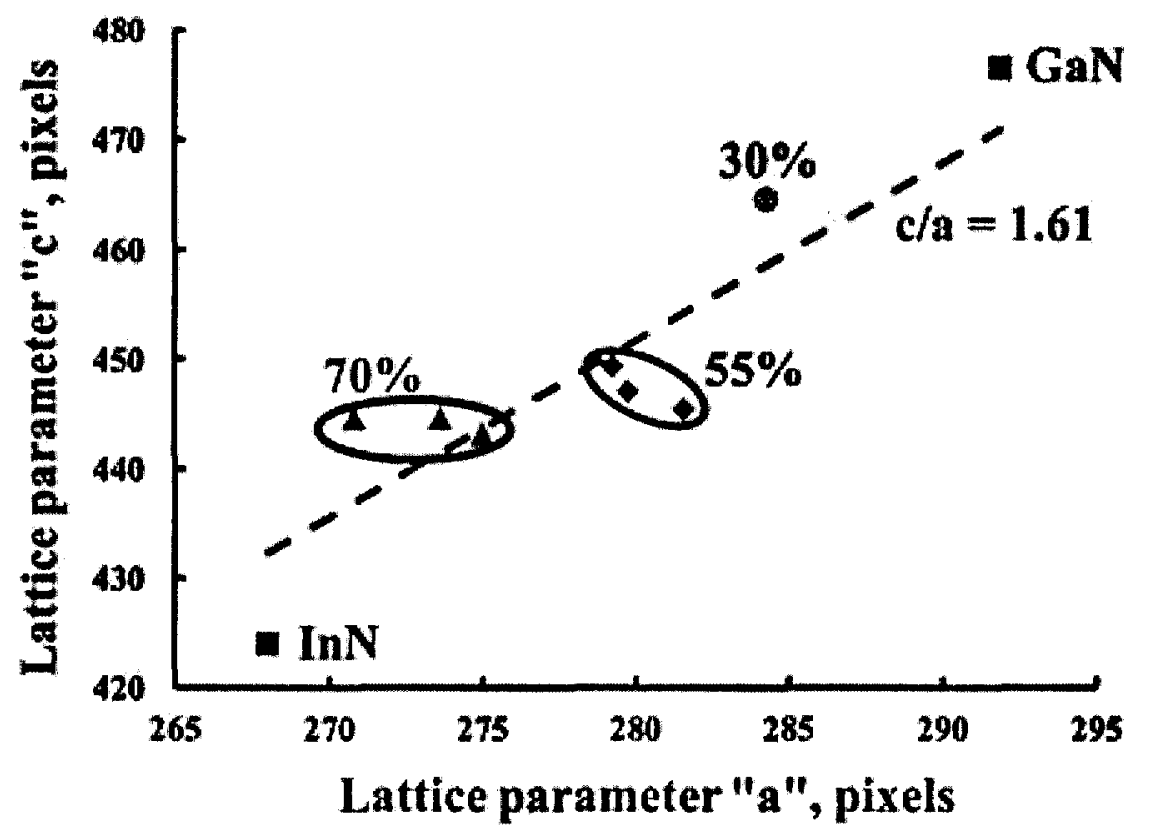

Figure 6.10 Measured lattice parameters of $\mathrm{InGaN}, \mathrm{GaN}$ and $\mathrm{InN}$ via CBED. The measured compositions show that on nanowires from one sample there is a minor composition variation and overall it matched with that measured from other techniques. All the measurements are consistent with the characteristic wurtzite c/a ratio 1.6.

\subsubsection{Precursor shielding effect}

In the CBED measurements, some composition variation was observed on the same wire along the length. This could be attributed to the non-uniform precursor distribution in the chamber from the showerhead and can be handled via tuning of the structural components. However, there is another very interesting parameter at work here. Figure 5.28 shows the extremely high density of the GaN nanowires. The density is 
similar on all the substrates. Although this high density is good for applications that need enhanced surface area, it is a problem when trying to grow uniform layers on the nanowires. The gradually increasing density from the top towards the substrate surface is clearly evident.

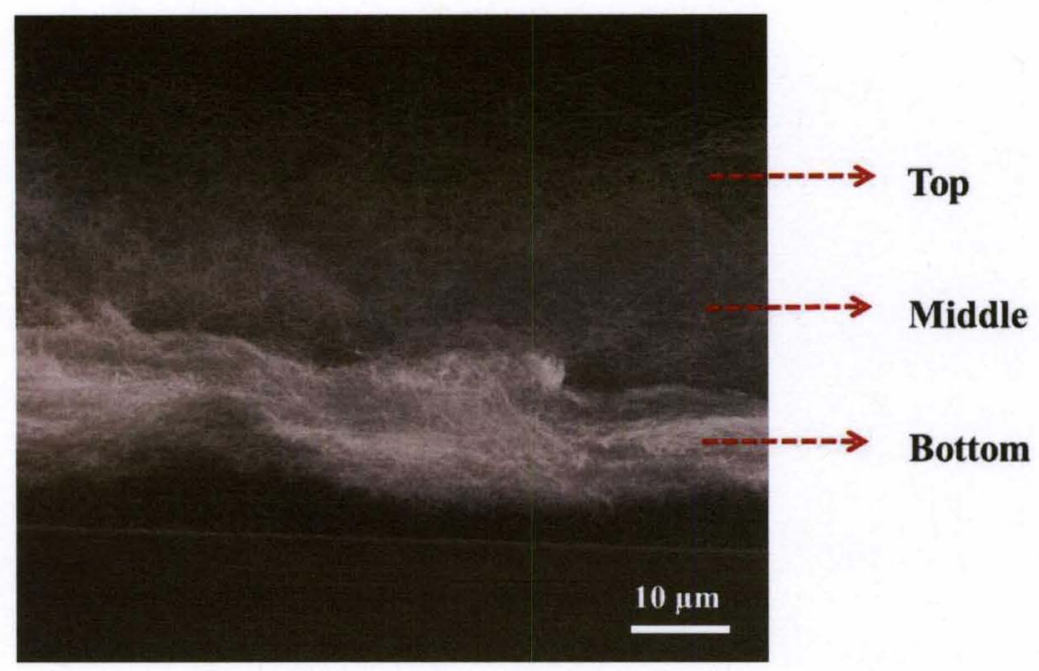

Figure 6.11 SEM micrograph of the extremely high density of GaN nanowires. The density gradient increasing from the top to the bottom (substrate surface) is also shown.

The extremely high density of nanowires can cause a shielding of the precursors to the nanowires in the layers below the surface. Growth at different heights was examined to study if there is in fact any precursor shielding by the nanowires. Figure 6.11 shows the growth on nanowires on the same substrate at different regions of the growth. Growth is completely conformal smooth shell like growth on the nanowires on the top as shown in figure 6.12a. Partial coverage is observed on the nanowires in the middle region as seen in figure $6.12 \mathrm{~b}$. Growth is almost non-existent with clean surface for nanowires at the bottom as shown in figure $6.12 \mathrm{c}$. 


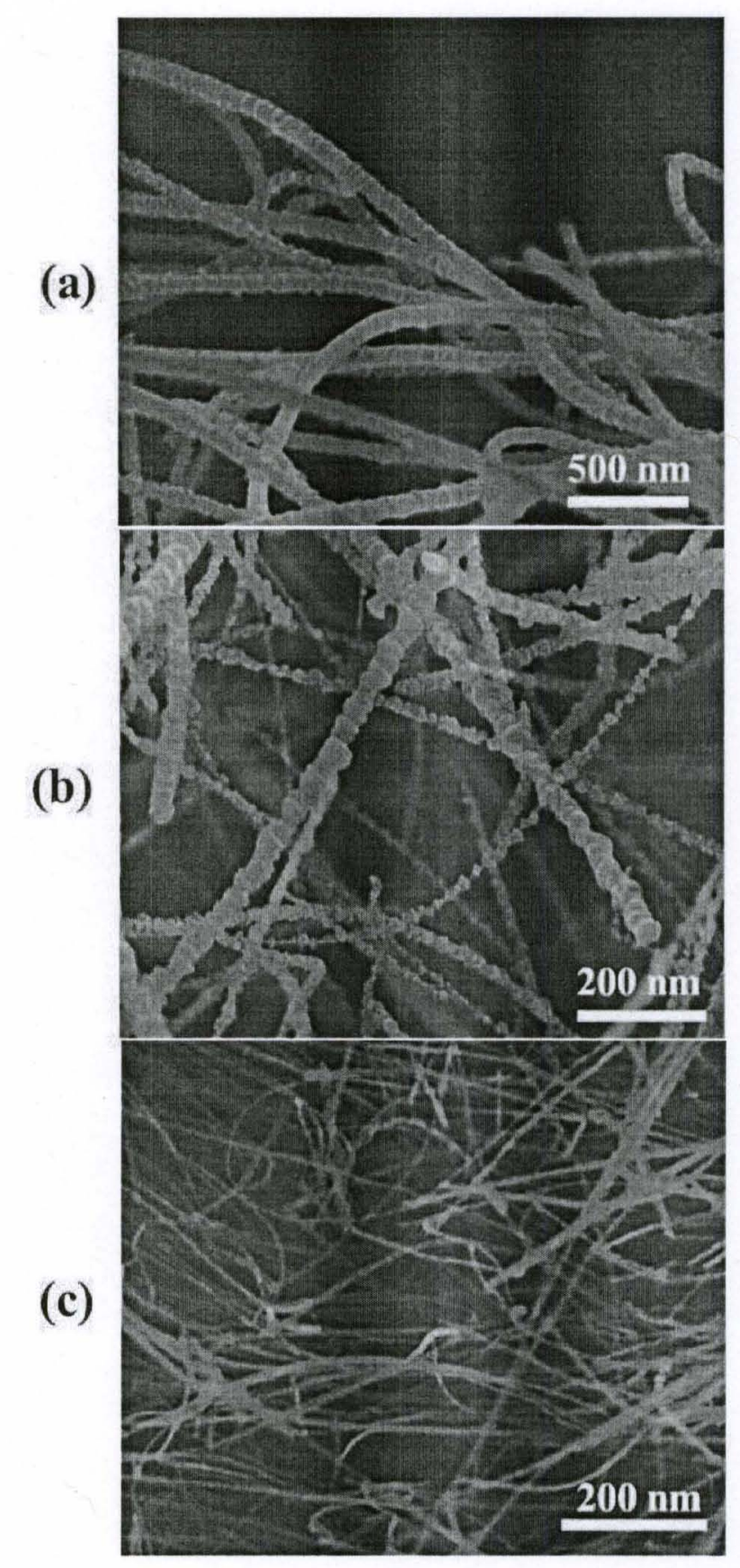

Figure 6.12 SEM micrographs of the variation in InGaN growth as a function of the distance from the top demonstrating the shielding of the precursor flux reaching the lower layers due to the high density of the nanowires. The wires on the top layer have a uniform conformal coating but the growth tends to be more scattered and island like in the middle 
regions. At the lower region, close to the substrate, there is practically no growth of InGaN.

Also, it can be seen that the nanowires do not remain in one plane but form a $3 \mathrm{~d}$ matrix. The wires bend and dive down from the top to the lower zones which along with the shielding effect could cause non uniform precursor supply causing the observed composition fluctuation along one wire. This is almost like a manifestation of the time evolution of growth shown in figure 6.1 where time was the control to reach full coverage starting from island formation. Here, the high density of nanowires cause shielding of the precursors and lead to a pseudo time delay in growth which as a window for understanding the evolution of the growth process. An interesting observation nonetheless!

\subsubsection{Orientation dependent heteroepitaxial growth of InGaN on GaN NWs}

The morphology of InGaN layers on c-GaN and a-GaN nanowires is distinctly unique as seen in figure 6.1. The time evolution shows the formation shows the formation of the scattered islands at the initial stages. The islands grow, coalesce forming a corrugated shell at first. Further growth occurs to fill up the valleys giving the shell a much smoother appearance. 


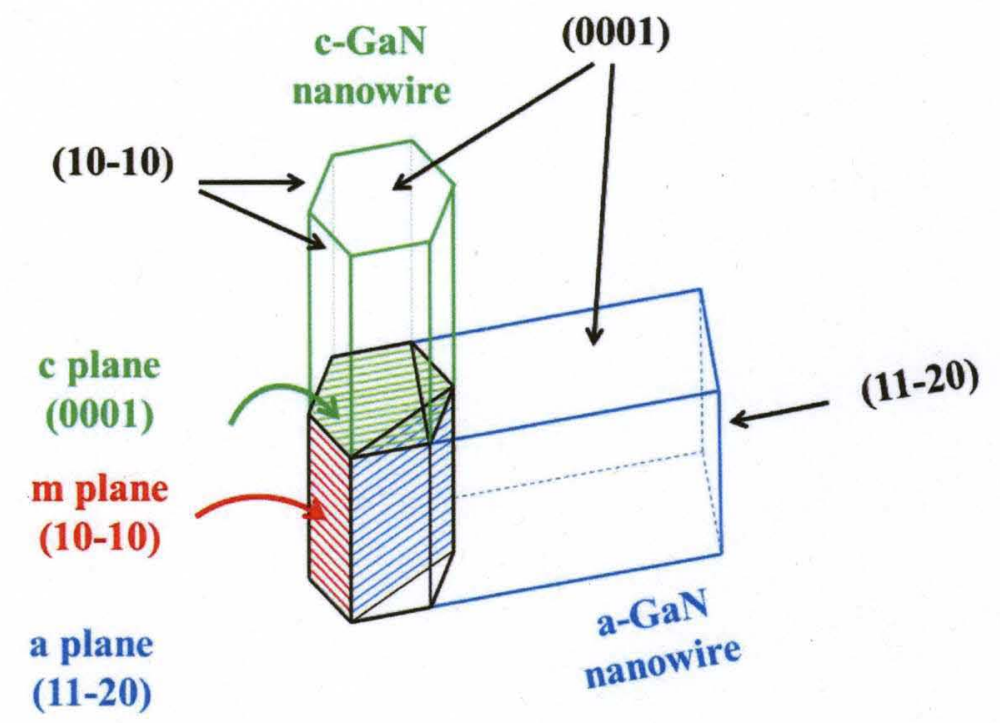

Figure 6.13 Schematic of the hexagonal GaN crystal structure. c-GaN nanowires have a hexagonal cross section bounded by six non polar (10-10) facets while a-GaN nanowires have a rectangular cross section bounded by alternating pairs of polar (0001) and nonpolar (10-10) facets.

Explaining the mechanism starts with a review of the crystal structure of GaN. As shown in figure 6.13, the $\mathrm{c}-\mathrm{GaN}$ nanowires (growing in the $<0001>$ direction) have a hexagonal cross section and are bound by the six nonpolar facets (10-10) while the a$\mathrm{GaN}$ nanowires (growing along the (11-20) plane) have a rectangular cross section bound by alternating polar (0001) and non-polar (10-10) facets. The difference in structure and polarity is expected to play a part in the observed morphological difference in InGaN growth.

InGaN growth on $\mathrm{GaN}$ is initiated by the formation of hexagonal islands. The base of the island is the polar (0001) plane while the six inclined facets are the semi-polar 
(10-11) planes. The epitaxial growth of c-planes is favored on c-planes of GaN nanowires. Hence, on c-GaN nanowires, the islands are aligned along the length of the nanowire (c-plane of the island grows epitaxially to the c-plane of GaN nanowire). It also explains the alignment of islands perpendicular to the length and also only on two opposite facets of the nanowire, as shown in figure $6.1 \mathrm{~d}$, in a-GaN nanowires that have only two (0001) facets.

Now that the orientation behavior is clarified, it is important to understand the growth mechanism of $\mathrm{InGaN}$ on $\mathrm{GaN}$ nanowires in order to determine the role of nanowires as strain relaxing substrates. Figure 6.14 shows the proposed growth mechanism of InGaN layers on GaN nanowires. The high flux is the reason for the island nucleation else growth would be layered at low flux conditions like in MBE. High flux conditions are also responsible for the small inter-island spacing of 30-50 $\mathrm{nm}$ whereas low flux conditions would result in large (microns scale) inter-island spacing ${ }^{173}$. The small spacing is also a reflection on the small adatom diffusion length on the nanowire surface as the diffusion process is constrained by the small diameters of the nanowires unlike thin films where the large unconstrained 2-D surfaces lead to larger diffusion lengths. As shown in figure $6.14 \mathrm{a}$, on a $\mathrm{c}-\mathrm{GaN}$ nanowire, since all the facets are similar, formation of a uniform symmetric step on all the facets starts the growth process. Since the step is epitaxial to the surface, it too has gallium and nitrogen terminated surfaces with different reactivity. The high reactivity of the gallium terminated surface ${ }^{181}$ causes material addition on that surface much faster than the nitrogen terminated surface. This leads to the growth of the step along one direction only. Also, the polarity effect from the nanowires leads to a directional adatom diffusion. Both these factors contribute to the 
observation of the islands being aligned in the same direction as shown in figure 6.1a. Similar directional growth of islands has been observed for homoepitaxial GaN growth on m-plane and (10-11) oriented substrates ${ }^{182}$. The directionality could be due to anisotropy originating from polarization created due to the $+\mathrm{c}$ and $-\mathrm{c}$ facets which would be accentuated in the case of nanowires.

(a)

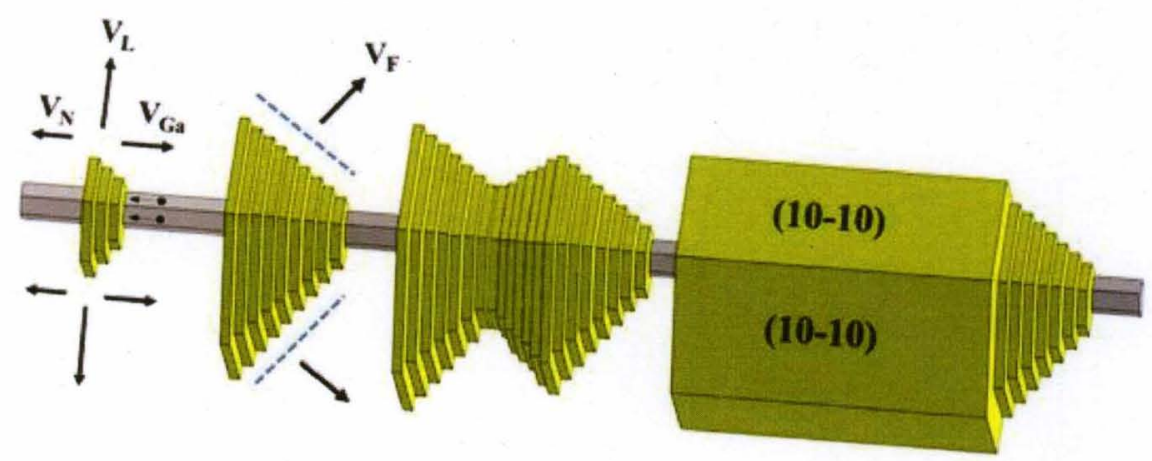

(b)

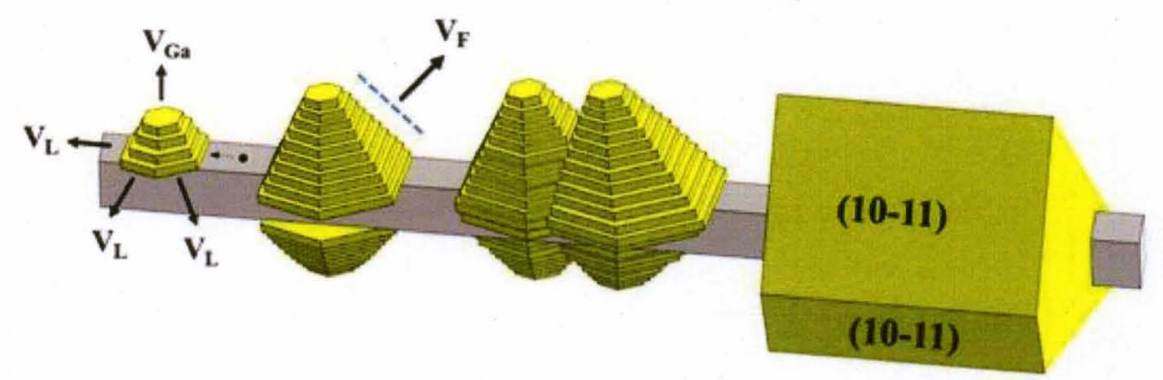

Figure 6.14 Schematic of the growth mechanism of InGaN growth on $\mathrm{GaN}$ nanowires. a) InGaN growth on a-GaN nanowires leads to a nonpolar hexagonal shell on the nanowire while b) growth on a-GaN nanowire leads to the formation of a semi-polar shell on the nanowire. 
Once a step is formed, growth in the axial direction occurs via adatom addition to the gallium terminated surface $\left(V_{G a} \gg V_{N}\right)$. The rate of nucleation of new steps on the existing layers competes with the rate of the step growth along the axis. The lateral growth rate of the base of the pyramid is comparable to the growth rate of the tip of the pyramid. The adatoms can hop from the new steps down to the older steps towards the base of the pyramid but cannot hop to the nitrogen terminated surface due to its low reactivity. The SE (Schwoebel Ehlrich) barrier ${ }^{183,184}$ prevents adatoms from hopping from the base towards the tip of the pyramid. The SE barrier has been defined as "the energetic unwillingness of atoms to descend step edges" ${ }^{185}$. In semiconductors, the barrier however has also been observed for adatoms moving up the steps ${ }^{186}$. The energetics of the material and the facets control the barrier direction. It has been observed that the direction of ES barrier can also affect the step flow to cause uniform steps or lead to step bunching ${ }^{186}$. The constant growth rates maintain the pyramidal shape of the growing islands. The formation of islands has been observed also in the case of heteroepitaxial growth of GaN growth on planar sapphire and AlN substrates and attributed to strain relaxed growth ${ }^{187}$. The difference between the growth on planar substrates and nanowires is the spatial constraint induced due to the small nanowire diameters. Hence, the effective adatom diffusion lengths are much smaller $(30-50 \mathrm{~nm})$ compared to those on planar substrates (microns).

Our data shown in figure 6.15 suggests that the island formation happens at the very initial stages of the growth with dimensions $<5 \mathrm{~nm}$. The small pyramids are also observed in the EDS maps in figure 6.9. Once a pyramid forms, the growth of the steps in the lateral and axial directions maintains the pyramidal aspect ratio and leads to an 
increase in the size of the islands that can be illustrated as the growth of the inclined facet with a constant velocity $\mathrm{V}_{\mathrm{F}}$. The islands grow unhindered till they come in contact with a neighboring island. Once the tip of an island comes in contact with the base of another island the axial growth process stops. Now, the adatoms act to fill up the valley leading to the coalescence of the islands. This causes the observation of growth to evolve from scattered islands to coalescence, finally leading to the formation of a smooth shell on the nanowire. Since, the growth is symmetric on the nanowire the InGaN shell also has a six fold symmetry bound by $(10-10)$ facets.

As shown in figure $6.14 \mathrm{~b}$, growth on $\mathrm{a}-\mathrm{GaN}$ is a little different. The "a" plane nanowires are bound by a pair of (0001) and (10-10) surfaces. Growth occurs on the two c planes as the islands have a hexagonal cross section due to the epitaxy with the underlying (0001) plane. The islands grow perpendicular to the length of the nanowire as shown in figure 6.1d. Analogous to growth on $\mathrm{c}-\mathrm{GaN}$ nanowires, step formation starts the nucleation process. The hexagonal step grows laterally via adatoms attaching on all the six nonpolar facets and new steps nucleate on the preexisting steps. The SE barrier prevents the adatoms from hopping up the steps leading to the formation of the pyramidal shape. The adatoms from the vapor phase as well as those diffusing on the nanowire surface contribute to the growth. The constant axial and lateral growth velocities lead to the growth of the pyramid and it seems that the inclined facet is growing at a constant velocity. As the size of the pyramid increases, the inter-pyramid distance decreases and finally the bases of adjacent pyramids come in contact. Now, the lateral growth stops. The adatoms act to fill up the valleys reducing the corrugated appearance and finally leading to the formation of a smooth shell. This shell is a result of the merging of the 
inclined (10-11) facets. Hence, the shell is bound by four semi-polar (10-11) surfaces. The formation of pyramids as mirror images on either side of the nanowires is very interesting. Growth starts via the formation of pyramid on the more reactive (0001) plane. The mirror pyramid grows via the formation of a pyramid on the underside of this pyramid. In both the cases, the final step where valleys get filled up is via homoepitaxial growth as the underlying layer is already InGaN. It has been shown that island growth has been shown to be a form of strain relaxed growth ${ }^{187}$ indicating that InGaN growth on GaN nanowires is strain relaxation process.

Although a smooth shell forms on the a-GaN and c-GaN nanowires with a similar island growth mechanism, the characteristics are very different. The shell on the c-GaN nanowires is hexagonal and is bounded by six non polar (10-10) facets. However, the InGaN shell on a-GaN nanowires has a square cross section and is bounded by four semi-polar (10-11) facets. This approach can be developed for polarity controlled synthesis of nanowires. InGaN as a material system has many applications and semi-polar nanowire architecture could lead to some interesting applications ${ }^{188}$.

Figure 6.15 is the SEM image of InGaN growth on "c" GaN nanowires showing the very small pyramids to show that it was indeed the observation. In depth analysis on the pyramidal island growth was performed to prove that the inclined facet indeed grew at a constant velocity. If this is the case, the aspect ratio of the islands irrespective of the size should be the same and equal to the wurtzite GaN aspect ratio of 1.63 . The aspect ratio of many islands on different samples was measured. However, the geometry of the crystals being measured has to be accounted for the correct measurement of the aspect ratio. The aspect ratio "c/a" is a measure of the length of one side of the hexagon to the 
height of the hexagon. As shown in the schematic in figure 6.16, the actual lengths measured are projections that change with the perspective. Geometrical correlations are made between the measured lengths and the actual aspect ratio. From the perspective where only two facets can be completely seen, the aspect ratio becomes 0.92 and is 0.81 when the three facets of the hexagonal pyramid can be seen.

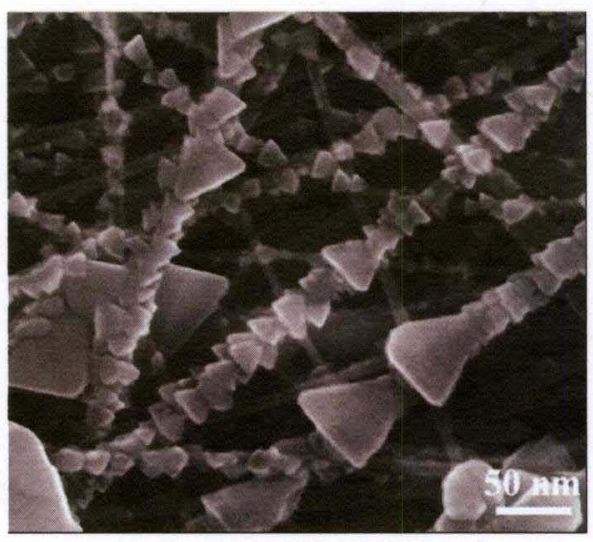

Figure 6.15 SEM micrograph of the InGaN growth on $\mathrm{GaN}$ nanowires. Islands with different sizes are shown indicating that the islands form at the very initial stages followed by the island growth at constant aspect ratio.

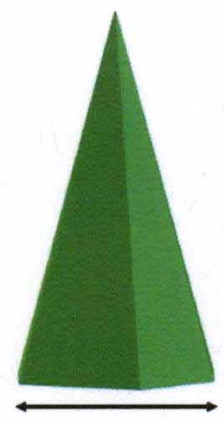

1.73 a

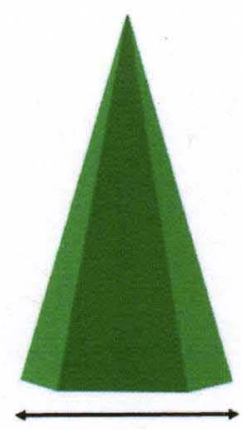

2 a

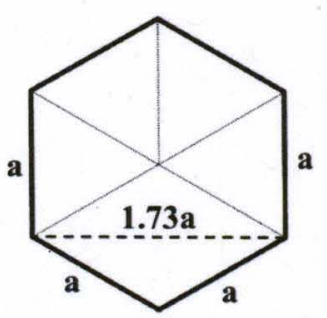

Figure 6.16 Schematic of the two possible views of the hexagonal pyramidal islands. The base is equal to 1.72 times the lattice constant "a" when only two facets are visible 
whereas the measured base would be twice the lattice constant "a" when three facets are visible.

The measured aspect ratios of the islands of various sizes are plotted in figure 6.17. All the islands fall into one of the two geometries indicating that the islands have a constant aspect ratio irrespective of the size.

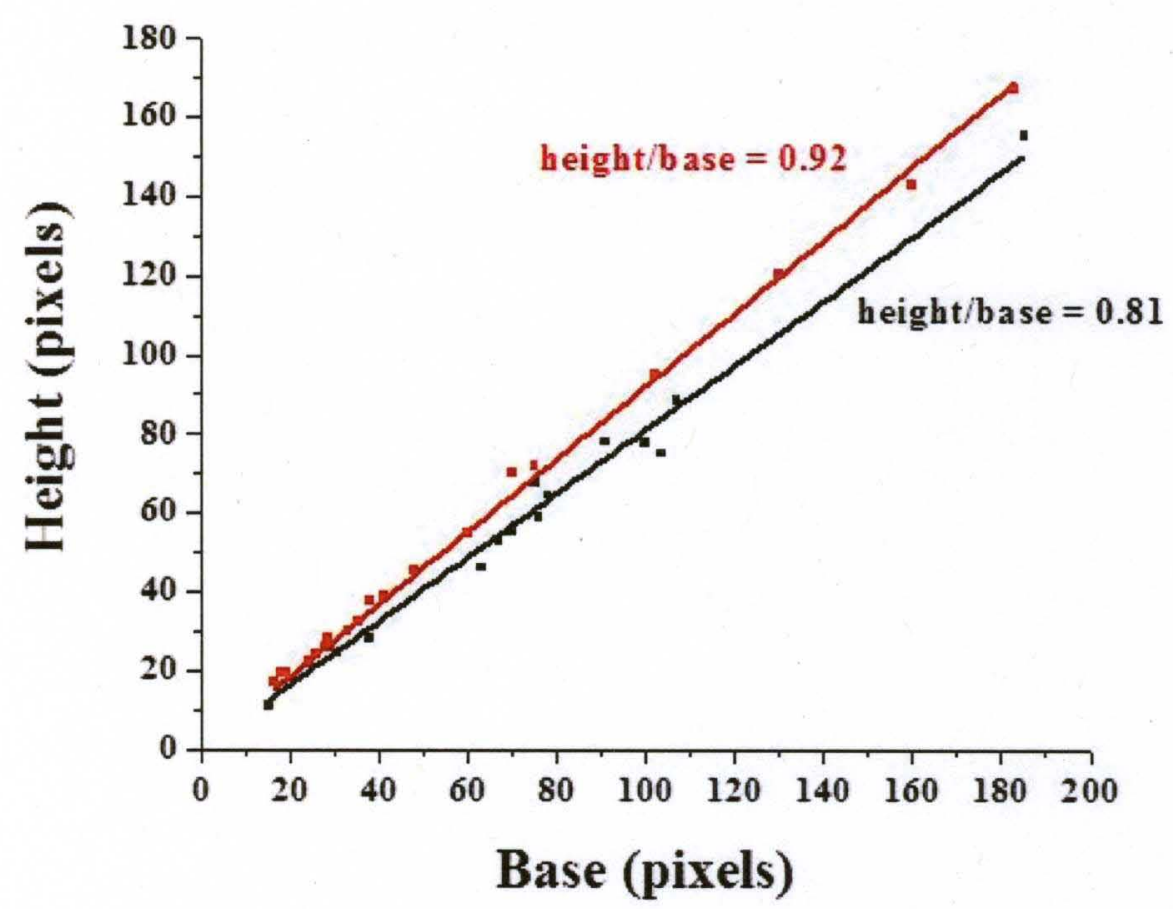

Figure 6.17 Aspect of the pyramidal islands of different sizes and different InGaN compositions. Number of facets of the pyramid visible in the plan view controls the ratio. The steps form a pyramidal shape at very initial stages and grow in size maintain the aspect ratio. The size of the pyramids has been measured from $<5 \mathrm{~nm}$ (smallest) to $>100$ $\mathrm{nm}$ (largest), corroborating our proposed growth mechanism. 


\subsection{Photoactivity of InGaN layers}

The final step in this study was to measure the photoactivity of the InGaN layers. GaN nanowires only absorb light in the UV range which is a small fraction of the solar spectrum. InGaN with a band gap of $2 \mathrm{eV}$ can absorb a large fraction of sun light increasing the amount of charge carriers generated and aiding in more efficient water splitting process. Here, we measure the voltage and current generated when the material absorbs sunlight.

\subsubsection{Illuminated OCP}

This is the simplest measure of the photoactivity of the material. Figure 6.17 shows the measured photo-voltage on the $\mathrm{GaN}$ nanowires and $\mathrm{InGaN}$ coated $\mathrm{GaN}$ nanowires. Upon illumination, in both cases, the potential becomes more negative which is an indication of the material being n-type. In figure $6.18 \mathrm{a}$, the generated photo-voltage is $\sim 20 \mathrm{mV}$ which is small. GaN is a wide band gap material and the AM1.5 sunlight has small amount of UV that is required for band edge absorption. Hence, the low photovoltage generation as the incident energy is very small to start with. The measurements were cycled from light to dark for about 10 minutes and the potentials remained stable indicating that there is no fast degradation of the material in solution.

The photovoltage in the InGaN coated GaN is also in the same order of magnitude. Since the precursor shielding did not allow for a complete coverage of the nanowires with InGaN, the system still is only GaN nanowires connected to the substrate 
which would result in the same voltage generation. InGaN has a lower conduction band than $\mathrm{GaN}$ which would prevent electron injection into $\mathrm{GaN}$ from $\mathrm{InGaN}$. We need a completely coated $\mathrm{GaN}$ nanowire with InGaN shell contacting the substrate where the electrons can go from InGaN to the back contact.
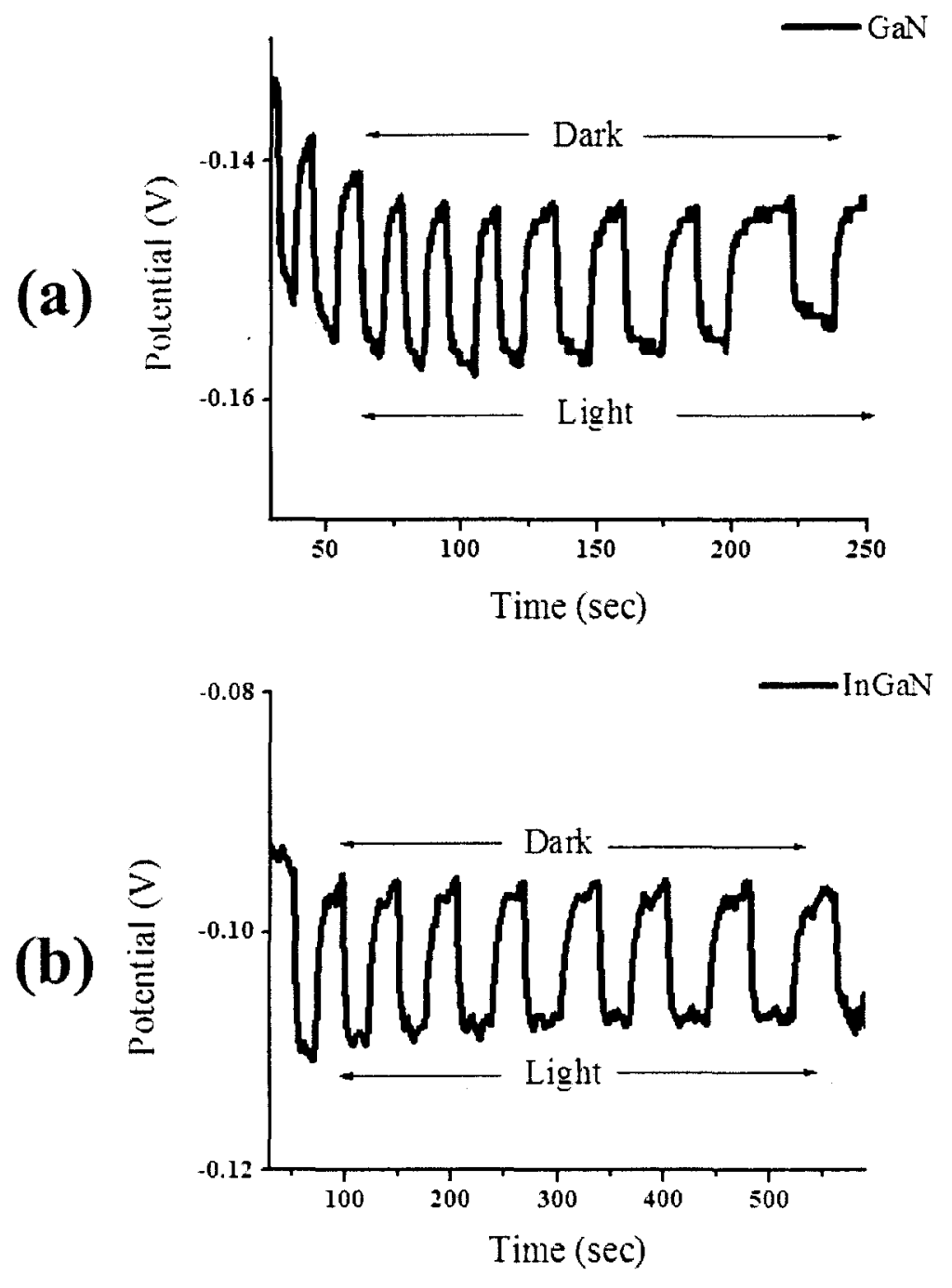

Figure 6.18 Illuminated open circuit potential (photo-voltage) measurements on GaN nanowires a) and GaN nanowires coated with $\mathrm{InGaN}$ layers b). Both show the potentials 
become more negative upon illumination which indicates the materials being n-type. The measured $\mathrm{OCP}$ is constant reflecting on the stability of the material.

It is clear that the 3-d mat like nanowire growth with high density is good when using GaN nanowires directly for applications. However, this is not the right architecture for $\mathrm{InGaN} / \mathrm{GaN}$ structures. Vertical arrays of $\mathrm{GaN}$ nanowires with reduced density are needed for our water splitting applications. The time of experiments and flux need to be tuned to get to vertical GaN nanowire arrays.

\subsubsection{Photocurrent}

Figure 6.19 is a plot of the current in dark and under illumination $\left(100 \mathrm{~mW} / \mathrm{cm}^{2}\right)$ measured on $\mathrm{InGaN}$ coated $\mathrm{GaN}$ nanowires. There is a definite increase in current with illumination which is a proof of the photoactivity of the InGaN/GaN. The photocurrent onset moved from about $0.35 \mathrm{~V}$ to $0.15 \mathrm{~V}$. The photocurrent is in the order of $0.07 \mathrm{~mA}$

(the electrode area was $0.9 \mathrm{~cm}^{2}$ ). This low current also points to the fact that this could be a response from $\mathrm{GaN}$ nanowires only. It is hence imperative to grow $\mathrm{InGaN}$ as a complete layer on $\mathrm{GaN}$ nanowire arrays to achieve the right device architecture. 


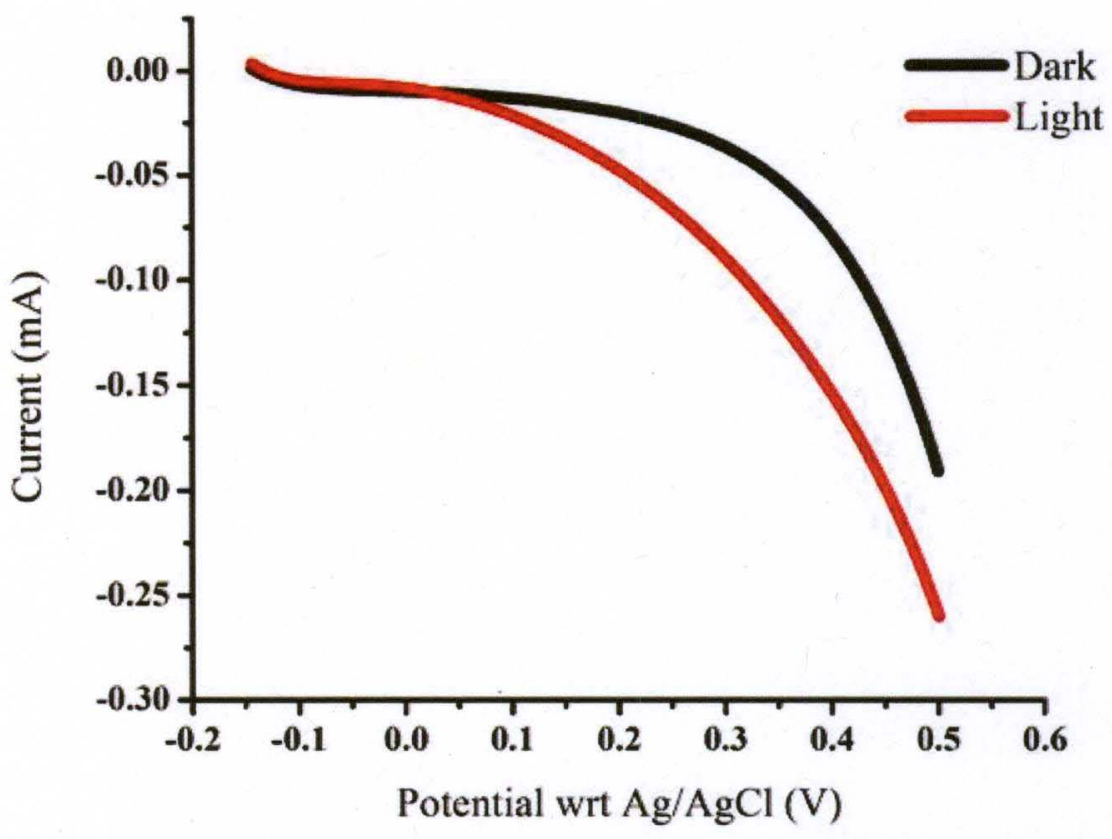

Figure 6.19 Photocurrent measurements on the $\mathrm{InGaN} / \mathrm{GaN}$ electrode. Potential scan was performed from $-0.05 \mathrm{~V}$ vs $\mathrm{OCP}$ to $0.5 \mathrm{~V}$ at a scan rate of $25 \mathrm{mV} / \mathrm{sec}$. Photocurrent onset occurs at about $0.1 \mathrm{~V}$ wrt $\mathrm{Ag} / \mathrm{AgCl}$. All the measurements were conducted at an illumination intensity of $100 \mathrm{~mW} / \mathrm{cm}^{2}$ 


\section{CHAPTER 7}

\section{GaSbN: A NOVEL MATERIAL SYSTEM}

\subsection{Introduction}

The right material for spontaneous photoelectrochemical water splitting has to satisfy the band gap, band edge positions and the stability criteria. To date, none of the materials being investigated have satisfied all the criteria. Apart from development of tandem cells, another approach is to search/design a new material that would be a potential PEC material. Recently, a $\left(\mathrm{Ga}_{1-\mathrm{x}} \mathrm{Zn}_{\mathrm{x}}\right)\left(\mathrm{N}_{1-\mathrm{x}} \mathrm{O}_{\mathrm{x}}\right)$ with $0.05<\mathrm{x}<0.22$ has been shown to have promising PEC characteristics. This system fares better than superlattice structures in terms of absorption in the long wavelength energies. The material has shown reasonable stability as well. However, theoretical estimations have shown that the

maximum band gap reduction was $2.6 \mathrm{eV}^{189}$, also measured experimentally ${ }^{94}$ indicating the material to be an inefficient absorber. Studies have also suggested than increasing $\mathrm{Zn}$ content in the alloy increases its photocatalytic activity ${ }^{63.190}$. Band gap reduction to the tune of $1 \mathrm{eV}$ has been demonstrated in the case of $\mathrm{ZnO}_{1-\mathrm{x}} \mathrm{Se}_{\mathrm{x}}$ with substitutional Se addition $\mathrm{x}<0.12$ but the stability of this alloy has not been studied. Analogous to $\mathrm{ZnO}$, 
GaN provides the right basis to start with for the development of ternary alloys for water splitting due to the stability and favorable band edge energetics.

$\mathrm{In}_{\mathrm{x}} \mathrm{Ga}_{1-\mathrm{x}} \mathrm{N}$ is one of the most widely studied materials for photoelectrochemical water splitting where about $40-60 \%$ indium content is desirable to get the right band gap. It has been shown theoretically ${ }^{43}$ and experimentally ${ }^{191}$ that such high indium content leads to instability in the alloy causing phase segregation. Interestingly, not much work has been focused on antimony based GaN alloys. There are reports on dilute nitrogen containing $\mathrm{GaSb}_{1-\mathrm{x}} \mathrm{N}_{\mathrm{x}}$ alloys where huge band gap bowing has been reported ${ }^{192,193}$ running into negative band gaps at $8 \% \mathrm{~N}^{194}$. There are no reports on the dilute antimony content alloys that are likely to show similar band gap bowing with interesting implications. The only studies in a similar concentration region were focused towards using antimony as a surfactant in GaN growth ${ }^{195,196}$ where some new below band gap emissions were observed. No systematic study of the change in band gap at dilute antimony compositions in $\mathrm{GaSb}_{\mathrm{x}} \mathrm{N}_{1-\mathrm{x}}$ alloys has been reported.

Theoretical modeling was employed to determine the electronic structure of the $\mathrm{GaSb}_{\mathrm{x}} \mathrm{N}_{1-\mathrm{x}}$ alloy at dilute antimony compositions ${ }^{197}$.

\subsection{Computational Model}

First principle density functional theory (DFT) calculations are performed using Cambridge Serial Total Energy Package (CASTEP) ${ }^{198}$ as implemented in the Materials Studio Software Package (Accelrys Software Inc.), based on local density approximation (LDA) and generalized gradient approximation (GGA). Ultrasoft pseudopotential ${ }^{199}$ is 
used for electron-ion interaction. The incorporation of the Hubbard parameter $U$ (which is an on-site Coulomb repulsion parameter) in the formalism (LDA/GGA $+U$ ) helps compensate for the well-known "gap problem" for the LDA/GGA formalism by incorporating a part of the electron correlation absent in LDA/GGA. The $U$ values are tuned by calculating the bulk GaN and GaSb band gap. This method GGA + U was used to calculate the band gap, density of states (DOS) and optical properties. The geometric structures in all calculations were optimized using GGA and the Perdew-Burke-Ernzerof (PBE) correlation function prior to performing the GGA + U calculations. Spin-polarized GGA + U calculations were performed using an energy cutoff of $600 \mathrm{eV}$. The imaginary part of the complex dielectric function from the matrix elements of the position operator between occupied and unoccupied states within the spin-polarized GGA $+U$ method implemented in CASTEP are used to calculate the optical spectra and the allowed transitions determined by the nonzero matrix elements of the position operator. For accurate determination of the optical properties, sufficient number of points is needed. Hence we used $5 \times 5 \times 5$ k-point grid. Also, we used 100 empty bands for accurate calculations of conduction bands. Convergence criteria were tested by changes in optical properties with respect to the number of $\mathrm{k}$ points and conduction bands.

A relatively large 72-atom supercell with periodic boundary conditions as shown in figure 7.1a was used for the bulk GaN wurtzite structure simulation. $\mathrm{GaSb}_{x} \mathrm{~N}_{1-\mathrm{x}}$ structures are obtained by substituting up to three $\mathrm{N}$ atoms with $\mathrm{Sb}$. In each case, both the cell volume and atom positions are optimized without any symmetry constraints. A 48atom supercell as shown in figure $7.1 \mathrm{~b}$ was considered for zinc blende bulk $\mathrm{GaSb}$ 
structure to study the effect of $\mathrm{N}$ incorporation into the lattice and also validate the model.

(a)

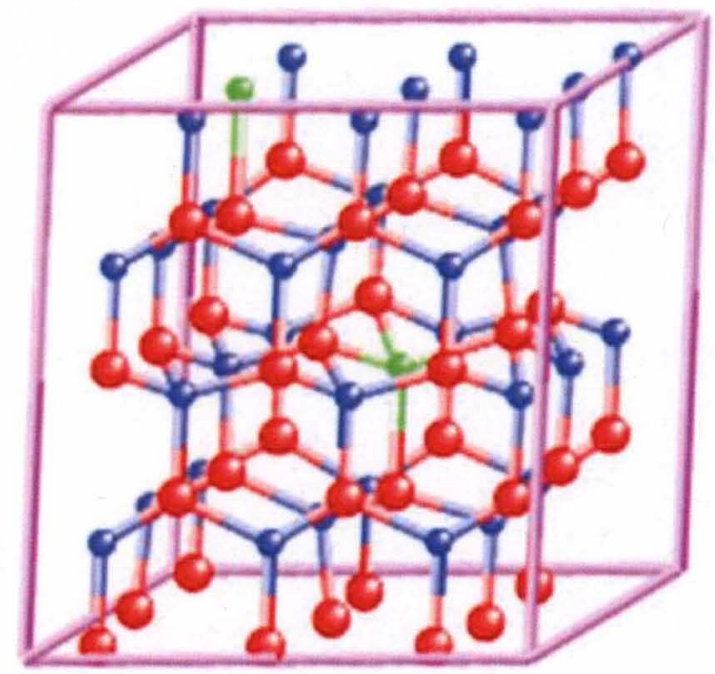

(b)

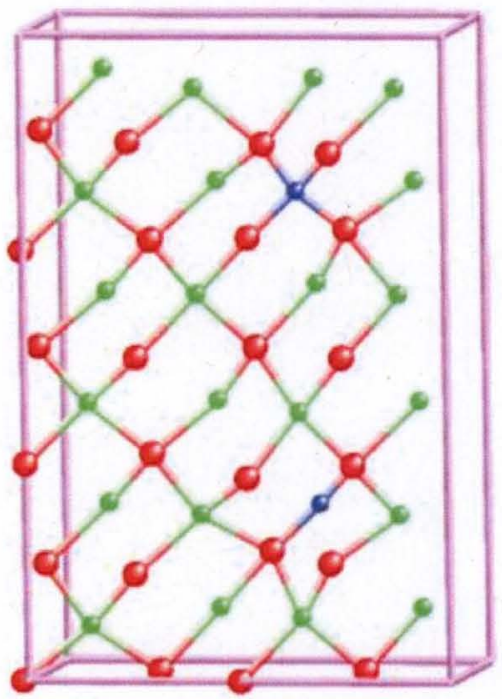

Figure 7.1 a) Optimized 72-atom wurtzite $\mathrm{GaN}$ supercell with two substitutional $\mathrm{Sb}$ atoms (in green). b) Optimized 48-atom zinc blende $\mathrm{GaSb}$ supercell with two substitutional $\mathrm{N}$ atoms (in blue) ${ }^{197}$.

The thermal stability analysis of the $\mathrm{GaSb}_{\mathrm{x}} \mathrm{N}_{1-\mathrm{x}}$ structures was performed using $a b$ initio molecular dynamic (MD) simulations on the Born-Oppenheimer potential surface using the Plane-Wave Self-Consistent Field (PWSCF) code of the QUANTUMESPRESSO package ${ }^{200}$. The interatomic forces were calculated using the HellmanFeynman theorem on the self-consistent electronic ground state at each time step, described by the DFT electronic structure, by solving the equations of motion with an integration time step of $1 \mathrm{fs}$. The simulation time of each trajectory is more than $2 \mathrm{ps}$, 
enough to explore structural changes. Since these alloys will be synthesized at temperatures close to $500 \mathrm{C}$, constant-temperature MD simulations were performed using the Berendsen thermostat ${ }^{201}$ at $800 \mathrm{~K}$ starting from the structures optimized in CASTEP.

\subsection{Simulation results}

The optimized structures shown in in figure 6.1 were optimized. Then, the high temperature MD simulations conducted to investigate the stability of the alloy showed no dissociation of the material indicating that the structure is completely stable at the temperatures of study.

Electronic structures of the optimized structures of $\mathrm{GaSb}_{x} \mathrm{~N}_{1-\mathrm{x}}$ and $\mathrm{GaSb}_{1-x} \mathrm{~N}_{\mathrm{x}}$ alloys were calculated. For the dilute antimony containing alloys $\mathrm{GaSb}_{x} \mathrm{~N}_{1-x}$ there is a monotonous increase in the lattice parameter while there is a monotonous decrease for dilute nitrogen containing $\mathrm{GaSb}_{1-\mathrm{x}} \mathrm{N}_{\mathrm{x}}$ alloys. Considerable local distortions in the lattice of the alloys occur due to the size and electronegativity mismatch between $\mathrm{N}$ and $\mathrm{Sb}$ atoms. Figure 7.2 shows the change in band gap observed with dilute additions of $\mathrm{N}$ in $\mathrm{GaSb}$ and $\mathrm{Sb}$ in $\mathrm{GaN}$ and the reported experimental band gaps for $\mathrm{GaSb}_{1-\mathrm{x}} \mathrm{N}_{\mathrm{x}}$ alloys $(\mathrm{x}=$

$0.015^{192}$, 202 and $\mathrm{x}=0.017^{203}$ ) to validate the calculations. The $\mathrm{GaSb}_{\mathrm{x}} \mathrm{N}_{1-\mathrm{x}}$ alloys show rapid decrease in the band gap with increasing $\mathrm{Sb}$ incorporation 


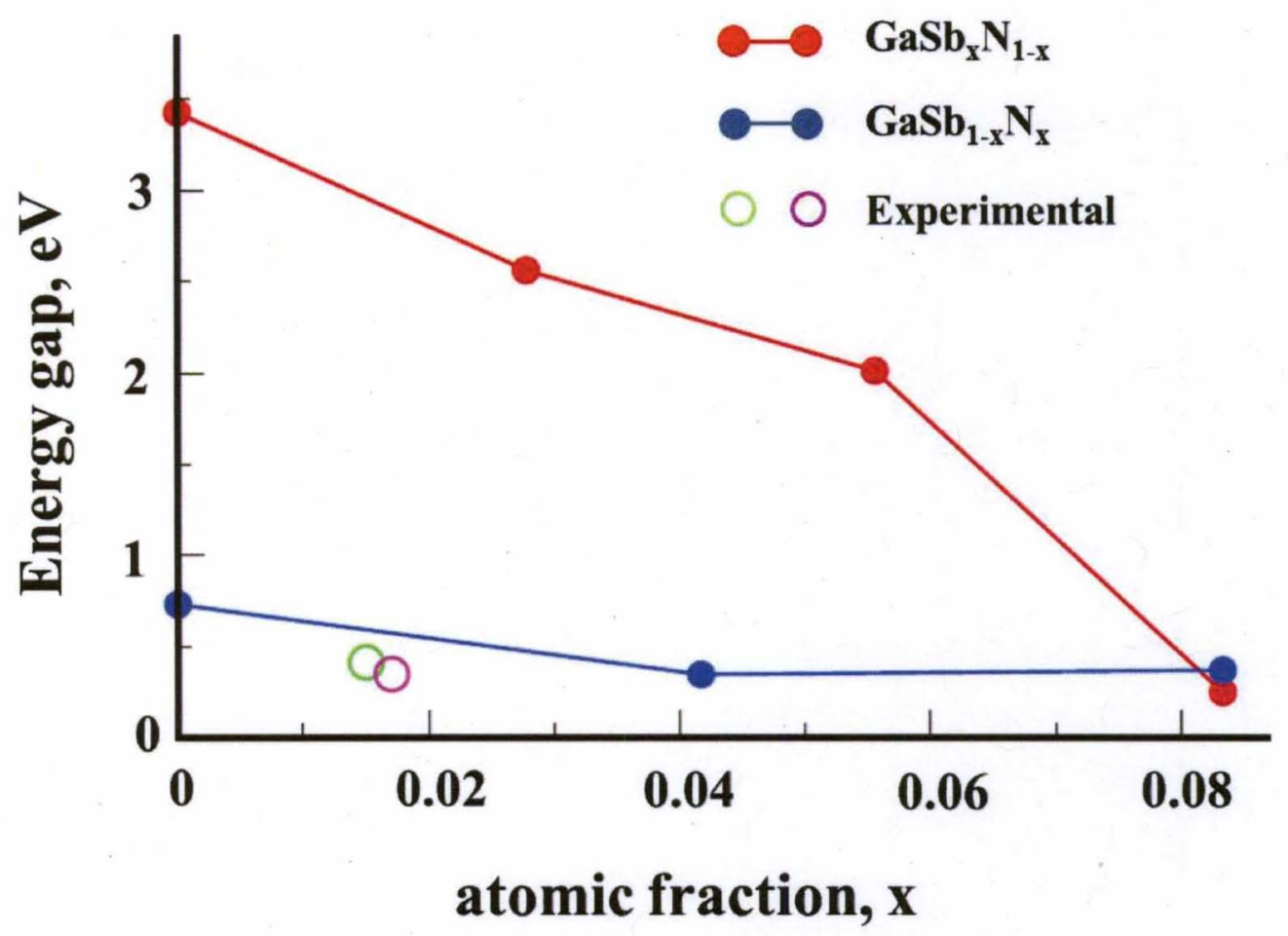

Figure 7.2 Calculated band gaps for $\mathrm{GaSb}_{\mathrm{x}} \mathrm{N}_{1-\mathrm{x}}$ and $\mathrm{GaSb}_{1-\mathrm{x}} \mathrm{N}_{\mathrm{x}}$ alloys as a function of concentration ${ }^{197}$. Experimentally reported band gap values for $\mathrm{GaSb}_{1-\mathrm{x}} \mathrm{N}_{\mathrm{x}}$ alloys are also included for comparison.

The large band gap reduction has also been observed for other highly mismatched alloys (HMAs) ${ }^{204}$. III-V hosts with isovalent $\mathrm{N}$ and As substituting group $\mathrm{V}$ anions are the most widely studied HMAs. The most successful model to explain this phenomenon is the band anticrossing (BAC) model. The BAC model incorporates an anticrossing interaction between localized states of the substitutional isovalent ions and the extended states of the host III-V semiconductor host. The BAC model is referred to as the conduction-BAC (CBAC) or valence-BAC (VBAC) depending on whether the impurity 
level is coupled to the conduction or the valence band respectively. The VBAC model has widely been employed to explain the band gap reduction for number of $\mathrm{HMAs}^{205}$.

Large sized impurities with first ionization energies less than that of the host anion have their defect states lay near the valence band edge of the host semiconductor. Antimony is among the largest of the group V species and its energies are near and below the valence band maximum (VBM) of GaN. The anticrossing interaction causes the splitting up of bands, restructuring the VBM i.e. shifting it upwards leading to a reduction in the band gap. The VBM shifts upwards at a rapid rate with increasing Sb concentration while conduction bands move down at a much slower rate.
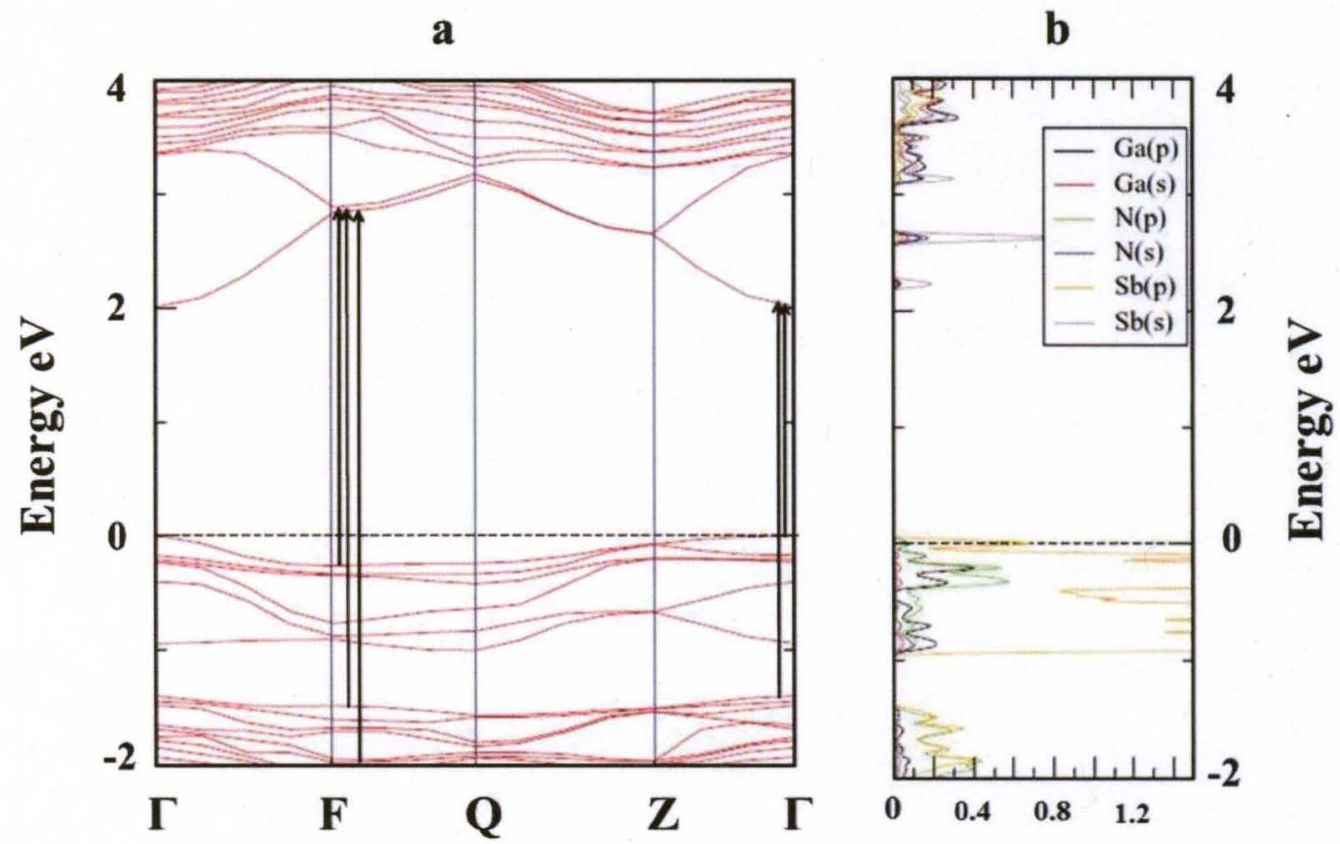

\section{PDOS}

Figure 7.3 a) Calculated band structure, allowed optical transitions and b) PDOS for the $\mathrm{GaSb}_{\mathrm{x}} \mathrm{N}_{1-\mathrm{x}}(0.05<\mathrm{x}<0.06)$ alloy ${ }^{197}$. 
Figure 7.3a shows the calculated band structure for the GaSbN alloys within the composition 0.05 and 0.06 . Splitting of the valence band is clearly evident. Also, as shown in the projected density of states (PDOS) plot in figure 7.4b, p states of $\mathrm{Sb}$ and the extended $s$ and $p$ states of the host are responsible for the narrowing of the band gap.

\subsection{Implications of the band gap reduction}

The band gap of $2 \mathrm{eV}$ obtained for $0.05<\mathrm{x}<0.06$ is has significant implications in the visible light absorption properties of the $\mathrm{GaSb}_{x} \mathrm{~N}_{1-x}$ alloys. Apart from the fact that the calculated band gaps are direct in nature, the huge band gap reduction to $2 \mathrm{eV}$ from $3.4 \mathrm{eV}$ with very little antimony incorporation makes this material system very interesting for light harvesting applications. However, for the material system to be suitable for PEC water splitting, the most important characteristic is the band energy positions.

Figure 7.4 shows the band edge positions of $\mathrm{GaSb}_{x} \mathrm{~N}_{1 \cdot x}$ alloy compositions along with those for $\mathrm{GaSb}^{34}$ and $\mathrm{InN}^{34}$ compared to the hydrogen and oxygen evolution potentials $(\mathrm{pH}=4)$. Since most of the bowing occurs in the valence band, the conduction band remains almost at the same energy. In the plot, the vacuum and electrochemical energy scales had to be unified. The relation used for the conversion from (standard hydrogen electrode) SHE to vacuum (vac) was

$$
E(S H E)+E(v a c)=-4.44
$$




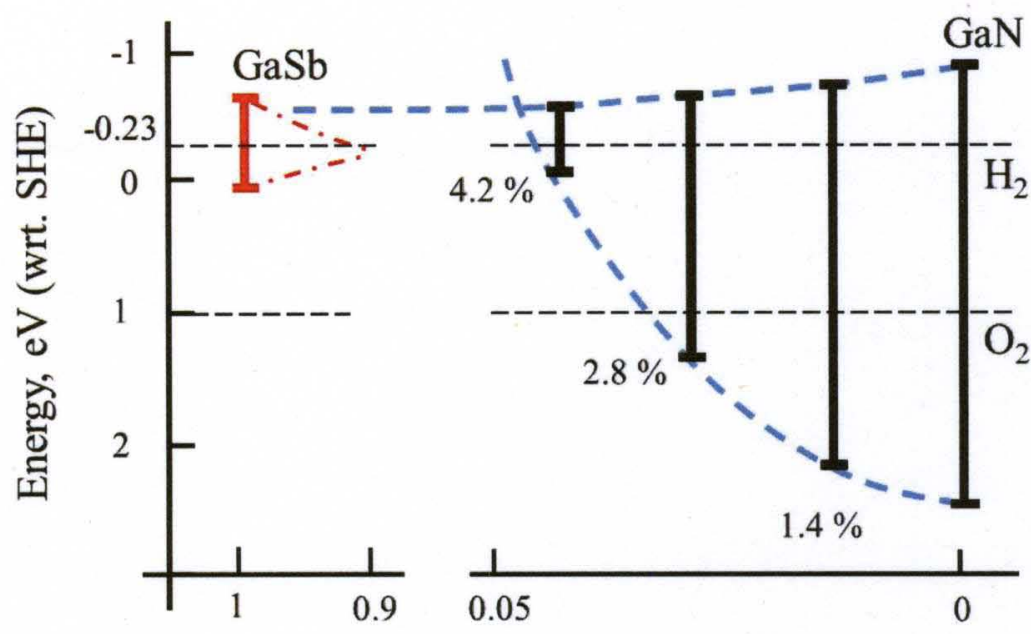

$\mathrm{x}$, alloying fraction $\left(\mathrm{In}_{\mathrm{x}} \mathrm{Ga}_{1-\mathrm{x}} \mathrm{N}\right.$ and $\left.\mathrm{GaSb}_{\mathrm{x}} \mathrm{N}_{1-\mathrm{x}}\right)$

Figure 7.4 A qualitative comparison of the band edge of $\mathrm{GaSbN}$ alloys wrt the hydrogen and oxygen water splitting potentials. The $\mathrm{GaSb}_{1-\mathrm{x}} \mathrm{N}_{\mathrm{x}}$ band edge positions are plotted using values from literature whereas the $\mathrm{GaSb}_{\mathrm{x}} \mathrm{N}_{1-\mathrm{x}}$ values are the calculated values in this study ${ }^{197}$. It is evident that the $\mathrm{GaSb}_{\mathrm{x}} \mathrm{N}_{1-\mathrm{x}}$ alloy straddles the water splitting reaction potentials.

The composition dependent band gap plot of $\mathrm{GaSb}_{\mathrm{x}} \mathrm{N}_{1-\mathrm{x}}$ clearly indicates that at $~$ $\mathrm{x}=0.06$, the band gap is $2 \mathrm{eV}$ and that the band edges straddle the $\mathrm{H}_{2} / \mathrm{O}_{2}$ potentials. The straddle is significant in both ends making this a very significant result. The material system is one of the most promising systems for spontaneous water splitting. The almost equal straddle should provide a uniform driving force for the water splitting reaction. Estimates show that the material should straddle the potentials at moderate (8) to low (1) $\mathrm{pH}$ values. 
The idea of this study was to see if novel alloys could be designed deriving from the excellent properties of $\mathrm{GaN}$. The addition of $6 \% \mathrm{Sb}$ is not expected to alter the stability of the alloy from GaN. Also, this system could be an alternative to the $(\mathrm{GaN})_{\mathrm{x}}(\mathrm{ZnO})_{1-\mathrm{x}}$ system which could suffer from charge imbalance resulting from non isovalent substitutions of $\mathrm{Ga}$ with $\mathrm{Zn}$ and $\mathrm{N}$ with $\mathrm{O}$. More importantly, local defect centers would be created that could act as charge recombination sites adversely affecting the PEC water splitting efficiency if the $\mathrm{N}$ atom is far away from $\mathrm{Ga}$ atom in the matrix.

Hence, GaSbN system is definitely promising and the results show it to be one of the more promising material systems to pursue for PEC water splitting. 


\section{CHAPTER 8}

\section{CONCLUSIONS}

In this dissertation, the role of nanowires to act as strain relaxing substrates for ternary material systems prone to phase segregation was investigated. GaN nanowires with diameters 15-30 nm with controlled orientation were synthesized to be used as nonrigid substrates. InGaN layers with controlled composition were synthesized on the GaN nanowires in a home built MOCVD reactor. Flow and reaction rate modeling was performed on the reactor to compare with the observed growth rates. Theoretical simulations were also performed on dilute antimony containing $\mathrm{GaSbN}$ alloys to check for the band gap and band edge positions.

\subsection{GaN nanowire synthesis}

- High density of GaN nanowires with controlled "a" plane and "c" plane orientation was synthesized on various metal (stainless steel and copper), insulator (quartz) and semiconductor (silicon and c-GaN) substrates with the same reaction parameters. 
- "a" plane nanowires were obtained during low temperature synthesis on metals (copper and stainless steel) and high temperature synthesis on insulating (quartz) substrates.

- "c" plane nanowires were obtained during high temperature synthesis on metals (copper and stainless steel) and semiconducting (silicon and c-GaN) substrates.

- Substrate temperature influences the wetting and nucleation behavior leading to the formation of nanowires.

- GaN nanowires can also be synthesized with copper catalyst with interesting morphologies. Continuous instability of the catalyst droplet leads to a coiled morphology and bent branches. Directed nanowire growth allows for the formation of straight nanowires for the growth of nanowire arrays.

\subsection{Reactor modeling}

- Growth rate increases with increase in temperature and precursor flow rates while a reduction in growth rate was observed with increase in pressure and separation of the substrate from the showerhead.

- A rapid decrease in growth rate was observed with increase in spacing from 0.5 to 1.5 inch and almost remains constant even at larger distances of 3 and 4.5 inches.

- A spacing of 1.5-2.5 inch between the showerhead and substrate was optimal in terms of growth rate and uniformity.

- Growth rate observed experimentally $(\sim 1 \mu \mathrm{m} / \mathrm{hr})$ was very similar to the $\mathrm{GaN}$ growth rate measured in the simulations $(\sim 600 \mathrm{~nm} / \mathrm{hr})$. 


\subsection{InGaN growth on GaN nanowires}

- InGaN growth started as scattered islands that coalesced into a uniform shell on the GaN nanowires.

- Single crystalline shell of InGaN with controlled composition was obtained only on $\mathrm{GaN}$ nanowires of diameters $<30 \mathrm{~nm}$. Thicker nanowires act as rigid planar substrates leading to polycrystalline growth.

- The very high density of GaN nanowires creates a screen, preventing growth on the nanowires in the lower levels.

\subsection{Photoactivity measurements}

- Bare GaN nanowires as well as InGaN on GaN show photovoltage.

- The InGaN layer is not continuous over the entire length of the nanowire. This could prevent carriers generated in the InGaN layers from reaching the back contact.

\subsection{Theoretical simulations of GaSbN}

- GaSbN alloy with $<10 \%$ antimony shows a large reduction in band gap from $3.4 \mathrm{eV}$ to $<2 \mathrm{eV}$.

- The band edges straddle the water redox potentials at moderate and low $\mathrm{pH}$.

- GaSbN alloys could be potential materials for PEC water splitting. 


\section{CHAPTER 9}

\section{RECOMMENDATIONS}

Growth of high density of GaN nanowire arrays is useful for studies involving GaN nanowires only. A low density growth of short GaN nanowire array is the ideal architecture for the epitaxial growth of $\mathrm{InGaN}$ on $\mathrm{GaN}$ nanowires. This would also be useful if $\mathrm{ALD}$ coating of $\mathrm{TiO}_{2}$ is needed to protect the metal from the electrolyte.

Automatic precursor flow controls would be ideal as manual controls can induce pressure variations that can alter the composition of the alloy. Also, in situ doping as well as use of more than two precursors at once would be possible. InGaN growth on short vertical arrays of GaN nanowires would be the ideal architecture for PEC water splitting.

GaSbN needs a very small antimony content $(<10 \%)$ to achieve the right band gap. Antimony has high vapor pressures which makes high temperature synthesis an issue where antimony desorbs out very easily. High pressure diamond anvil cells where GaN powder and antimony powder will be reacted at high temperatures and high pressures for the incorporation of antimony into GaN.

MOCVD growth of GaSbN using a low temperature nitrogen precursor can be employed. Since ammonia has high activation temperatures for GaN growth, alternative precursors which readily react at low temperatures to form GaN (hence InGaN) can be 
used. This would allow for a low temperature synthesis of the GaSbN alloy where antimony could be retained without evaporation. 


\section{REFERENCES}

1. Smil, V., Energy in the twentieth century: Resources, Conversions, Costs, Uses, and Consequences. Annual Review of Energy and the Environment 2000, 25, 31.

2. Energy, Published by the Office of Sciences and US Department of Energy, April 18 - 25. Basic Research needs for Solar Energy Utilization 2005.

3. Government, E. I. A.-O. E. S. f. t. U. G., International Energy Outlook 2008, Energy Information Brochures, DOE-EIA-X042.

4. http://www.worldcoal.org/coal/where-is-coal-found/.

5. http://www.bp.com/sectionbodycopy.do?categoryld=7500\&contentId=7068481 .

6. http://www.theglobaleducationproject.org/earth/energy-supply.php.

7. http://www.census.gov/main/www/popclock.html.

8. http://www.census.gov/population/international/data.

9. http://en.wikipedia.org/wiki/World_energy_consumption.

10. http://www.epa.gov/climatechange/emissions/co2_human.html.

11. http://www.epa.gov/climatechange/emissions/globalghg.html.

12. http://en.wikipedia.org/wiki/File:Global_Carbon_Emission_by_Type.png.

13. http://en.wikipedia.org/wiki/Greenhouse_gas.

14. Energy, P. b. t. O. o. S. a. U. D. o. E., Basic Research needs for Solar Energy Utilization, April 18-21, 2005.

15. http://en.wikipedia.org/wiki/Geothermal_energy. 
16. Exergy (available energy) Flow Charts.

17. Valcav Smil - Energy at the Crossroads.

18. Renewable Resource Data Center - PV Correction Factors.

19. http://en.wikipedia.org/wiki/Concentrated_solar_power.

20. Turner, J. A., Sustainable Hydrogen Production. Science 2004, 305 (5686), $972-$ 974.

21. Verne, J., The Mysterious Island (available at http://www.literatureweb.net/verne/mysteriousisland) 1874.

22. P, H., The Forever Fuel: The Story of Hydrogen (Westview Press, Boulder,CO) 1981.

23. Gregory, D., Sci. Am. 228, (1), 131973.

24. A realistic look at hydrogen price projections.

25. Vehicle Technologies Program: Fact \# 205: Frbruary 25, 2002 Hydrogen Cost and Worldwide Production eere.energy.gov.

26. Navarro Yerga, R. M.; Alvarez Galvan, M. C.; del Valle, F.; Villoria de la Mano, J. A.; Fierro, J. L. G., Water Splitting on Semiconductor Catalysts under Visible-Light Irradiation. Chemsuschem 2009, 2 (6), 471-485.

27. Navarro, R. M.; del Valle, F.; Villoria de la Mano, J. A.; Álvarez-Galván, M. C.; Fierro, J. L. G., Photocatalytic Water Splitting Under Visible Light: Concept and Catalysts Development. In Advances in Chemical Engineering, Hugo, I. d. L.; Benito Serrano, R., Eds. Academic Press: 2009; Vol. Volume 36, pp 111-143.

28. www.h2powersystems.com. 
29. Bak, T.; Nowotny, J.; Rekas, M.; Sorrell, C. C., Photo-electrochemical hydrogen generation from water using solar energy. Materials-related aspects. International Journal of Hydrogen Energy 2002, 27 (10), 991-1022.

30. Fujishima, A.; Honda, K., Electrochemical Photolysis of Water at a Semiconductor Electrode. Nature 1972, 238 (5358), 37-38.

31. http://en.wikipedia.org/wiki/File:Solar_Spectrum.png.

32. Nozik, A. J., PHOTOELECTROCHEMISTRY - APPLICATIONS TO SOLARENERGY CONVERSION. Annual Review of Physical Chemistry 1978, 29, 189-222.

33. Xu, Y.; Schoonen, M. A. A., The absolute energy positions of conduction and valence bands of selected semiconducting minerals. Am. Miner. 2000, 85 (3-4), 543-556.

34. Van de Walle, C. G.; Neugebauer, J., Universal alignment of hydrogen levels in semiconductors, insulators and solutions. Nature 2003, 723 (6940), 626-628.

35. Deutsch, T. G.; Koval, C. A.; Turner, J. A., III-V nitride epilayers for photoelectrochemical water splitting: GaPN and GaAsPN. Journal of Physical Chemistry B 2006, 110 (50), 25297-25307.

36. Bolton, J. R.; Strickler, S. J.; Connolly, J. S., Limiting and realizable efficiencies of solar photolysis of water. Nature 1985, 316 (6028), 495-500.

37. Walter, M. G.; Warren, E. L.; McKone, J. R.; Boettcher, S. W.; Mi, Q. X.; Santori, E. A.; Lewis, N. S., Solar Water Splitting Cells. Chem. Rev. 2010, 110 (11), 6446-6473.

38. Khaselev, O.; Turner, J. A., A Monolithic Photovoltaic-Photoelectrochemical Device for Hydrogen Production via Water Splitting. Science 1998, 280 (5362), 425-427. 
39. Beach, J. D.; Collins, R. T.; Turner, J. A., Band-Edge Potentials of n-Type and pType GaN. Journal of the Electrochemical Society 2003, 150 (7), A899-A904.

40. Nozik, A. J., P-N PHOTOELECTROLYSIS CELLS. Applied Physics Letters 1976, $29(3), 150-153$.

41. Liu, C.; Hwang, Y. J.; Jeong, H. E.; Yang, P., Light-Induced Charge Transport within a Single Asymmetric Nanowire. Nano Letters 2011, 11 (9), 3755-8.

42. Kayes, B. M.; Atwater, H. A.; Lewis, N. S., Comparison of the device physics principles of planar and radial p-n junction nanorod solar cells. Journal of Applied Physics 2005, 97 (11).

43. Ho, I.-h.; Stringfellow, G. B., Solid phase immiscibility in GaInN. Applied Physics Letters 1996, 69 (18), 2701-2703.

44. Karpov, S. Y., Suppression of phase separation in InGaN due to elastic strain. MRS Internet Journal of Nitride Semiconductor Research 1998, 3 (16).

45. Cho, H. K.; Yang, G. M., Generation of misfit dislocations in high indium content InGaN layer grown on GaN. Journal of Crystal Growth 2002, 243 (1), 124-128.

46. Tsao, J. Y., Materials Fundamentals of Molecular Beam Epitaxy. Adademic press: 1992; p 301 .

47. Tessarek, C.; Figge, S.; Aschenbrenner, T.; Bley, S.; Rosenauer, A.; Seyfried, M.; Kalden, J.; Sebald, K.; Gutowski, J.; Hommel, D., Strong phase separation of strained In_ $\{\mathrm{x}\} \mathrm{Ga} \_\{1-\mathrm{x}\} \mathrm{N}$ layers due to spinodal and binodal decomposition: Formation of stable quantum dots. Physical Review B 2011,83 (11), 115316. 
48. El-Masry, N. A.; Piner, E. L.; Liu, S. X.; Bedair, S. M., Phase separation in InGaN grown by metalorganic chemical vapor deposition. Applied Physics Letters 1998, $72(1), 40-42$.

49. Li, Q.; Wang, G. T., Strain influenced indium composition distribution in GaN/InGaN core-shell nanowires. Applied Physics Letters 2010, 97 (18).

50. Ertekin, E.; Greaney, P. A.; Chrzan, D. C.; Sands, T. D., Equilibrium limits of coherency in strained nanowire heterostructures. AIP: 2005; Vol. 97, p 114325.

51. Xiang, H. J.; Wei, S.-H.; Da Silva, J. L. F.; Li, J., Strain relaxation and band-gap tunability in ternary $\operatorname{In}(\mathrm{x}) \mathrm{Ga}(1-\mathrm{x}) \mathrm{N}$ nanowires. Physical Review B 2008, 78 (19).

52. Boettcher, S. W.; Warren, E. L.; Putnam, M. C.; Santori, E. A.; Turner-Evans, D.; Kelzenberg, M. D.; Walter, M. G.; McKone, J. R.; Brunschwig, B. S.; Atwater, H. A.; Lewis, N. S., Photoelectrochemical Hydrogen Evolution Using Si Microwire Arrays. Journal of the American Chemical Society 2011, 133 (5), 1216-1219.

53. Wang, G.; Ling, Y.; Wheeler, D. A.; George, K. E. N.; Horsley, K.; Heske, C.; Zhang, J. Z.; Li, Y., Facile Synthesis of Highly Photoactive alpha-Fe(2)O(3)-Based Films for Water Oxidation. Nano Letters 2011, 11 (8), 3503-3509.

54. Shi, J.; Hara, Y.; Sun, C.; Anderson, M. A.; Wang, X., Three-Dimensional HighDensity Hierarchical Nanowire Architecture for High-Performance Photoelectrochemical Electrodes. Nano Letters 2011, 11 (8), 3413-3419.

55. Skold, N.; Karlsson, L. S.; Larsson, M. W.; Pistol, M. E.; Seifert, W.; Tragardh, J.; Samuelson, L., Growth and optical properties of strained GaAs-GaxIn1-xP core-shell nanowires. Nano Letters 2005, 5 (10), 1943-1947. 
56. Kuykendall, T.; Ulrich, P.; Aloni, S.; Yang, P., Complete composition tunability of InGaN nanowires using a combinatorial approach. Nat Mater 2007, 6 (12), 951-956.

57. Hahn, C.; Zhang, Z.; Fu, A.; Wu, C. H.; Hwang, Y. J.; Gargas, D. J.; Yang, P., Epitaxial Growth of InGaN Nanowire Arrays for Light Emitting Diodes. ACS Nano 2011, $5(5), 3970-3976$.

58. Kim, H. M.; Lee, H.; Kim, S. I.; Ryu, S. R.; Kang, T. W.; Chung, K. S., Formation of InGaN nanorods with indium mole fractions by hydride vapor phase epitaxy. Physica Status Solidi B-Basic Research 2004, 241 (12), 2802-2805.

59. Johnson, M. A. L.; Hughes, W. C.; Rowland, W. H.; Cook, J. W.; Schetzina, J. F.; Leonard, M.; Kong, H. S.; Edmond, J. A.; Zavada, J., Growth of GaN, InGaN, and AlGaN films and quantum well structures by molecular beam epitaxy. Journal of Crystal Growth 1997, 175, 72-78.

60. Nakamura, S., InGaN-based violet laser diodes. Semiconductor Science and Technology 1999, 14 (6), R27-R40.

61. Qian, F.; Gradecak, S.; Li, Y.; Wen, C. Y.; Lieber, C. M., Core/multishell nanowire heterostructures as multicolor, high-efficiency light-emitting diodes. Nano Letters 2005, 5 (11), 2287-2291.

62. Maeda, K.; Takata, T.; Hara, M.; Saito, N.; Inoue, Y.; Kobayashi, H.; Domen, K., GaN:ZnO Solid Solution as a Photocatalyst for Visible-Light-Driven Overall Water Splitting. Journal of the American Chemical Society 2005, 127 (23), 8286-8287.

63. Chen, H.; Wang, L.; Bai, J.; Hanson, J. C.; Warren, J. B.; Muckerman, J. T.; Fujita, E.; Rodriguez, J. A., In Situ XRD Studies of ZnO/GaN Mixtures at High Pressure 
and High Temperature: Synthesis of $\mathrm{Zn}-\mathrm{Rich}(\mathrm{Ga} 1-\mathrm{xZnx})(\mathrm{N1} 1-\mathrm{xOx})$ Photocatalysts. The Journal of Physical Chemistry C 2010,114 (4), 1809-1814.

64. Aryal, K.; Pantha, B. N.; Li, J.; Lin, J. Y.; Jiang, H. X., Hydrogen generation by solar water splitting using p-InGaN photoelectrochemical cells. Applied Physics Letters 2010, $96(5), 052110$.

65. Kambe, S.; Fujii, M.; Kawai, T.; Kawai, S.; Nakahara, F., Photocatalytic hydrogen production with $\mathrm{Cd}(\mathrm{S}, \mathrm{Se})$ solid solution particles: Determining factors for the highly efficient photocatalyst. Chemical Physics Letters 1984, 109 (1), 105-109.

66. Frame, F. A.; Carroll, E. C.; Larsen, D. S.; Sarahan, M.; Browning, N. D.; Osterloh, F. E., First demonstration of CdSe as a photocatalyst for hydrogen evolution from water under UV and visible light. Chemical Communications 2008, (19), 22062208.

67. Sivula, K.; Le Formal, F.; Graetzel, M., WO(3)-Fe(2)O(3) Photoanodes for Water Splitting: A Host Scaffold, Guest Absorber Approach. Chemistry of Materials 2009, 21 (13), 2862-2867.

68. Balberg, I.; Pinch, H. L., The optical absorption of iron oxides. Journal of Magnetism and Magnetic Materials 7 (1-4), 12-15.

69. Kennedy, J. H.; Frese, K. W., PHOTO-OXIDATION OF WATER AT ALPHAFE2O3 ELECTRODES. Journal of the Electrochemical Society 1978, 125 (5), 709-714.

70. Chouhan, N.; Yeh, C. L.; Hu, S. F.; Huang, J. H.; Tsai, C. W.; Liu, R. S.; Chang, W. S.; Chen, K. H., Array of CdSe QD-Sensitized ZnO Nanorods Serves as Photoanode for Water Splitting. Journal of the Electrochemical Society 2010, 157 (10), B1430B1433. 
71. Chouhan, N.; Yeh, C. L.; Hu, S.-F.; Liu, R.-S.; Chang, W.-S.: Chen, K.-H., Photocatalytic CdSe QDs-decorated ZnO nanotubes: an effective photoelectrode for splitting water. Chemical Communications 2011, 47 (12), 3493-3495.

72. Knappenberger, K. L.; Wong, D. B.; Xu, W.; Schwartzberg, A. M.; Wolcott, A.; Zhang, J. Z.; Leone, S. R., Excitation-Wavelength Dependence of Fluorescence Intermittency in CdSe Nanorods. ACS Nano 2008, 2 (10), 2143-2153.

73. Wang, G.; Yang, X.; Qian, F.; Zhang, J. Z.; Li, Y., Double-Sided CdS and CdSe Quantum Dot Co-Sensitized ZnO Nanowire Arrays for Photoelectrochemical Hydrogen Generation. Nano Letters 2010, 10 (3), 1088-1092.

74. Smotkin, E. S.; Cervera-March, S.; Bard, A. J.; Campion, A.; Fox, M. A.; Mallouk, T.; Webber, S. E.; White, J. M., Bipolar cadmium selenide/cobalt(II) sulfide semiconductor photoelectrode arrays for unassisted photolytic water splitting. The Journal of Physical Chemistry 1987, 91 (1), 6-8.

75. Cho, S.; Jang, J.-W.; Kim, J.; Lee, J. S.; Choi, W.; Lee, K.-H., Three-Dimensional Type II $\mathrm{ZnO} / \mathrm{ZnSe}$ Heterostructures and Their Visible Light Photocatalytic Activities. Langmuir 2011, 27 (16), 10243-10250.

76. Sivula, K.; Le Formal, F.; Gratzel, M., Solar Water Splitting: Progress Using Hematite (alpha-Fe(2)O(3)) Photoelectrodes. Chemsuschem 2011, 4 (4), 432-449.

77. Kleiman-Shwarsctein, A.; Huda, M. N.; Walsh, A.; Yan, Y. F.; Stucky, G. D.; Hu, Y. S.; Al-Jassim, M. M.; McFarland, E. W., Electrodeposited Aluminum-Doped alpha$\mathrm{Fe}(2) \mathrm{O}(3)$ Photoelectrodes: Experiment and Theory. Chemistry of Materials 2010, 22 (2), 510-517. 
78. Kay, A.; Cesar, I.; Graetzel, M., New benchmark for water photooxidation by nanostructured alpha-Fe2O3 films. Journal of the American Chemical Society 2006, 128 (49), 15714-15721.

79. Ling, Y.; Wang, G.; Wheeler, D. A.; Zhang, J. Z.; Li, Y., Sn-Doped Hematite Nanostructures for Photoelectrochemical Water Splitting. Nano Letters 2011, II (5), 2119-2125.

80. Mao, A.; Park, N.-G.; Han, G. Y.; Park, J. H., Controlled growth of vertically oriented hematite/Pt composite nanorod arrays: use for photoelectrochemical water splitting. Nanotechnology 2011, 22 (17), 175703.

81. Le Formal, F.; Tetreault, N.; Cornuz, M.; Moehl, T.; Graetzel, M.; Sivula, K., Passivating surface states on water splitting hematite photoanodes with alumina overlayers. Chemical Science 2011, 2 (4), 737-743.

82. Yang, X.; Wolcott, A.; Wang, G.; Sobo, A.; Fitzmorris, R. C.; Qian, F.; Zhang, J. Z.; Li, Y., Nitrogen-Doped ZnO Nanowire Arrays for Photoelectrochemical Water Splitting. Nano Letters 2009, 9 (6), 2331-2336.

83. Khan, S. U. M.; Al-Shahry, M.; Ingler, W. B., Efficient Photochemical Water Splitting by a Chemically Modified n-TiO2. Science 2002, 297 (5590), 2243-2245.

84. Cole, B.; Marsen, B.; Miller, E.; Yan, Y.; To, B.; Jones, K.; Al-Jassim, M., Evaluation of nitrogen doping of tungsten oxide for photoelectrochemical water splitting. Journal of Physical Chemistry C 2008, 112 (13), 5213-5220.

85. Huda, M. N.; Al-Jassim, M. M.; Turner, J. A., Mott insulators: An early selection criterion for materials for photoelectrochemical H2 production. AIP: 2011; Vol. 3, p 053101. 
86. Mo, C. L.; Fang, W. Q.; Pu, Y.; Liu, H. C.; Jiang, F. Y., Growth and characterization of InGaN blue LED structure on Si(111) by MOCVD. Journal of Crystal Growth 2005, 285 (3), 312-317.

87. Sheu, J. K.; Chang, S. J.; Kuo, C. H.; Su, Y. K.; Wu, L. W.; Lin, Y. C.; Lai, W. C.; Tsai, J. M.; Chi, G. C.; Wu, R. K., White-light emission from near UV InGaN-GaN LED chip precoated with blue/green/red phosphors. IEEE Photonics Technol. Lett. 2003, $15(1), 18-20$.

88. Neufeld, C. J.; Toledo, N. G.; Cruz, S. C.; Iza, M.; DenBaars, S. P.; Mishra, U. K., High quantum efficiency InGaN/GaN solar cells with $2.95 \mathrm{eV}$ band gap. Applied Physics Letters 2008, 93 (14).

89. Jani, O.; Ferguson, I.; Honsberg, C.; Kurtz, S., Design and characterization of GaN/InGaN solar cells. Applied Physics Letters 2007, 91 (13).

90. Aryal, K.; Pantha, B. N.; Li, J.; Lin, J. Y.; Jiang, H. X., Hydrogen generation by solar water splitting using p-InGaN photoelectrochemical cells. Applied Physics Letters $2010,96(5)$

91. Li, J.; Lin, J. Y.; Jiang, H. X., Direct hydrogen gas generation by using InGaN epilayers as working electrodes. AIP: 2008; Vol. 93, p 162107.

92. Khaselev, O.; Turner, J. A., Electrochemical stability of p-GaInP2 in aqueous electrolytes toward photoelectrochemical water splitting. Journal of the Electrochemical Society 1998, $145(10), 3335-3339$.

93. Wang, H.; Deutsch, T.; Turner, J. A., Direct water splitting under visible light with nanostructured hematite and WO3 photoanodes and a GaInP2 photocathode. Journal of the Electrochemical Society 2008, 155 (5), F91-F96. 
94. Maeda, K.; Teramura, K.; Takata, T.; Hara, M.; Saito, N.; Toda, K.; Inoue, Y.; Kobayashi, H.; Domen, K., Overall Water Splitting on $($ Gal-xZnx $)(N 1-x O x)$ Solid Solution Photocatalyst: Relationship between Physical Properties and Photocatalytic Activity. The Journal of Physical Chemistry B 2005, 109 (43), 20504-20510.

95. http://www.ioffe.ru/SVA/NSM/Semicond/GaN/index.html.

96. Cai, X. M.; Ye, F.; Jing, S. Y.; Zhang, D. P.; Fan, P.; Xie, E. Q., CVD growth of InGaN nanowires. Journal of Alloys and Compounds 2009, 467 (1-2), 472-476.

97. Cai, X. M.; Leung, Y. H.; Cheung, K. Y.; Tam, K. H.; Djurisic, A. B.; Xie, M. H.; Chen, H. Y.; Gwo, S., Straight and helical InGaN core-shell nanowires with a high In core content. Nanotechnology 2006, 17 (9), 2330-2333.

98. Ye, F.; Cai, X. M.; Wang, X. M.; Xie, E. Q., The growth and field electron emission of InGaN nanowires. Journal of Crystal Growth 2007, 304 (2), 333-337.

99. Ok, J. E.; Jo, D. W.; Jeon, H. S.; Lee, A. R.; Lee, G. S.; Kim, K. H.; Ahn, H. S.; Yang, M., Structural Change of InGaN Nanostructures Grown by Mixed-Source Hydride Vapor Phase Epitaxy. Japanese Journal of Applied Physics 2011, 50 (1).

100. Nanishi, Y.; Saito, Y.; Yamaguchi, T.; Hori, M.; Matsuda, F.; Araki, T.; Suzuki, A.; Miyajima, T., MBE-growth, characterization and properties of $\operatorname{InN}$ and InGaN. Physica Status Solidi a-Applied Research 2003, 200 (1), 202-208.

101. Kurouchi, M.; Araki, T.; Naoi, H.; Yamaguchi, T.; Suzuki, A.; Nanishi, Y., Growth and properties of In-rich InGaN films grown on (0001) sapphire by RF-MBE. Physica Status Solidi B-Basic Research 2004, 241 (12), 2843-2848.

102. Pozina, G.; Bergman, J. P.; Monemar, B.; Mamutin, V. V.; Shubina, T. V.; Vekshin, V. A.; Toropov, A. A.; Ivanov, S. V.; Karlsteen, M.; Willander, M., Optical and 
structural characterization of $\mathrm{Ga}(\mathrm{In}) \mathrm{N}$ three-dimensional nanostructures grown by plasma-assisted molecular beam epitaxy. Physica Status Solidi B-Basic Research 1999, $216(1), 445-450$.

103. Kikuchi, A.; Kawai, M.; Tada, M.; Kishino, K., InGaN/GaN multiple quantum disk nanocolumn light-emitting diodes grown on (111)Si substrate. Japanese Journal of Applied Physics Part 2-Letters \& Express Letters 2004, 43 (12A), L1524-L1526.

104. Kouno, T.; Kikuchi, A.; Kishino, K., Growth of high-In-content InGaN multiple quantum disk nanocolumns on $\mathrm{Si}(111)$ by $\mathrm{RE}$ plasma-assisted molecular-beam epitaxy. Physica Status Solidi B-Basic Solid State Physics 2006, 243 (7), 1481-1485.

105. Morel, A.; Gallart, M.; Taliercio, T.; Lefebvre, P.; Gil, B.; Allegre, J.; Mathieu, H.; Damilano, B.; Grandjean, N.; Massies, J., Time-resolved spectroscopy of MBEgrown InGaN/GaN self-formed quantum dots. Physica Status Solidi a-Applications and Materials Science 2000, 180 (1), 375-380.

106. Egawa, T.; Zhang, B.; Ishikawa, H., High performance of InGaN LEDs on (111) silicon substrates grown by MOCVD. Ieee Electron Device Letters 2005, 26 (3), 169171.

107. Keller, S.; Abare, A. C.; Minsky, M. S.; Wu, X. H.; Mack, M. P.; Speck, J. S.; Hu, E.; Coldren, L. A.; Mishra, U. K.; DenBaars, S. P., MOCVD growth and properties of InGaN/GaN multi-quantum wells. In Silicon Carbide, Iii-Nitrides and Related Materials, Pts 1 and 2, Pensl, G. M. H. M. B. J. E., Ed. 1998; Vol. 264-2, pp 1157-1160. 108. Yang, P.-F.; Jian, S.-R.; Lai, Y.-S.; Yang, C.-S.; Chen, R.-S., Morphological, structural, and mechanical characterizations of InGaN thin films deposited by MOCVD. Journal of Alloys and Compounds 2008, 463 (1-2), 533-538. 
109. Moon, Y. T.; Kim, D. J.; Song, K. M.; Lee, I. H.; Yi, M. S.; Noh, D. Y.; Choi, C. J.; Seong, T. Y.; Park, S. J., Optical and structural studies of phase separation in InGaN film grown by MOCVD. Physica Status Solidi B-Basic Research 1999, 216 (1), 167-170. 110. Yam, F. K.; Hassan, Z., InGaN: An overview of the growth kinetics, physical properties and emission mechanisms. Superlattices and Microstructures 2008, 43 (1), 123.

111. Zhou, Y. G.; Zhang, R.; Li, W. P.; Shen, B.; Chen, P.; Chen, Z. Z.; Gu, S. L.; Shi, Y.; Zheng, Y. D.; Huang, Z. C., Gas-phase parasitic reactions and Al incorporation efficiency in light radiation heating, low-pressure metal-organic chemical vapor deposition of AlGaN. Materials Letters 2000, 45 (6), 331-335.

112. Theodoropoulos, C.; Mountziaris, T. J.; Moffat, H. K.; Han, J., Design of gas inlets for the growth of gallium nitride by metalorganic vapor phase epitaxy. Journal of Crystal Growth 2000, 217 (1-2), 65-81.

113. Nagatomo, T.; Kuboyama, T.; Minamino, H.; Omoto, O., PROPERTIES OF GA1-XINXN FILMS PREPARED BY MOVPE. Japanese Journal of Applied Physics Part 2-Letters 1989, 28 (8), L1334-L1336.

114. E.L. Piner, F. G. M., J.C. Roberts, M.E. Aumer, V.A. Joshkin, S.M. Bedair and N.A. El-Masry, MRS Internet J. Nitride Semicond. Res 1996, I.

115. Waltereit, P.; Brandt, O.; Ploog, K. H.; Tagliente, M. A.; Tapfer, L., Indium Surface Segregation during Growth of (In,Ga)N/GaN Multiple Quantum Wells by Plasma-Assisted Molecular Beam Epitaxy. physica status solidi (b) 2001, 228 (1), 49-53. 
116. Matsuoka, T.; Yoshimoto, N.; Sasaki, T.; Katsui, A., Wide-gap semiconductor InGaN and InGaAln grown by MOVPE. Journal of Electronic Materials 1992, 21 (2). 157-163.

117. Off, J.; Kniest, A.; Vorbeck, C.; Scholz, F.; Ambacher, O., Influence of buffer layers on the In-content of GaInN layers. Journal of Crystal Growth 1998, 195 (1-4), 286-290.

118. W. Van der Stricht, I. M., P. Demeester, L. Considine, E.J. Thrush and J.A. Crawley, MRS Internet J. Nitride Semicond. Res 1997, 2.

119. Kim, H.-M.; Lee, H.; Kim, S. I.; Ryu, S. R.; Kang, T. W.; Chung, K. S., Formation of InGaN nanorods with indium mole fractions by hydride vapor phase epitaxy. physica status solidi (b) 2004, $2+1$ (12), 2802-2805.

120. Oliver, R. A.; Kappers, M. J.; Humphreys, C. J.; Briggs, G. A. D., The influence of ammonia on the growth mode in InGaN/GaN heteroepitaxy. Journal of Crystal Growth 2004, 272 (1-4), 393-399.

121. Kim, D. J.: Moon, Y. T.; Song, K. M.; Lee, I. W.; Park. S. J., Effect of growth pressure on indium incorporation during the growth of InGaN by MOCVD. Journal of Electronic Materials 2001, 30 (2), 99-102.

122. Mahajan, S., materials, properties and preparation. amsterdam, New York: North-Holland, 1994.

123. Zhang, Q. L.; Meng, F. Y.; Crozier, P. A.; Newman, N.; Mahajan, S., Effects of stress on phase separation in $\operatorname{In}(\mathrm{x}) \mathrm{Ga}(1-\mathrm{x}) \mathrm{N} / \mathrm{GaN}$ multiple quantum-wells. Acta Mater. 2011, $59(10), 3759-3769$.

124. http://www.webelements.com/indium/atom_sizes.html. 
125. http://www.webelements.com/gallium/atom_sizes.html.

126. http://www.mt-berlin.com/frames_cryst/descriptions/sapphire.htm.

127. M.B.Santos, W. K. L. a., Thin Films: Heteroepitaxial System. World Scientific Publishing Co. Pte.Ltd: 1999.

128. Johnson, H. T.; Freund, L. B., Mechanics of coherent and dislocated island morphologies in strained epitaxial material systems. Journal of Applied Physics 1997, 81 (9), 6081-6090.

129. Joyce, P. B.; Krzyzewski, T. J.; Bell, G. R.; Joyce, B. A.; Jones, T. S., Composition of InAs quantum dots on $\mathrm{GaAs}(001)$ : Direct evidence for (In,Ga)As alloying. Physical Review B 1998, 58 (24), R15981.

130. Chen, H. J.; Feenstra, R. M.; Northrup, J.; Neugebauer, J.; Greve, D. W., Indium incorporation and surface segregation during InGaN growth by molecular beam epitaxy: experiment and theory. MRS Internet Journal of Nitride Semiconductor Research 2001, 6 (11), art. no.-11.

131. Rao, M.; Kim, D.; Mahajan, S., Compositional dependence of phase separation in InGaN layers. Applied Physics Letters 2004, 85 (11), 1961-1963.

132. K. Hiramatsu; Y. Kawaguchi; M. Shimizu; N. Sawaki; T. Zheleva; R. F. Davis; H. Tsuda; W. Taki; Kuwano, N.; and; Oki, K., MRS Internet Journal of Nitride Semiconductor Research 1997, 2 (6).

133. Lee, S.-N.; Sakong, T.; Lee, W.; Paek, H.; Seon, M.; Lee, I.-H.; Nam, O.; Park, Y., Characterization of optical and crystal qualities in InxGa1-xN/InyGal-yN multiquantum wells grown by MOCVD. Journal of Crystal Growth 2003, 250 (1-2), 256-261. 
134. Srinivasan, S.; Geng, L.; Liu, R.; Ponce, F. A.; Narukawa, Y.; Tanaka, S., Slip systems and misfit dislocations in InGaN epilayers. Applied Physics Letters 2003, 83 (25), 5187-5189.

135. Cho, H. K.; Lee, J. Y.; Yang, G. M.; Kim, C. S., Formation mechanism of V defects in the InGaN/GaN multiple quantum wells grown on $\mathrm{GaN}$ layers with low threading dislocation density. Applied Physics Letters 2001, 79 (2), 215-217.

136. Jahnen, B.; Albrecht, M.; Dorsch, W.; Christiansen, S.; Strunk, H. P.; Hanser, D.; Davis, R. F., Pinholes, dislocations and strain relaxation in InGaN. MRS Internet Journal of Nitride Semiconductor Research 1998, 3 (39), art. no.-39.

137. Sugahara, T.; Hao, M.; Wang, T.; Nakagawa, D.; Naoi, Y.; Nishino, K.; Sakai, S., Phase separation mechanism around dislocation in an $\mathrm{InGaN} / \mathrm{GaN}$ quantum well structure. In Compound Semiconductors 1998, Sakaki, H. W. J. C. Y. N. H. Y., Ed. 1999; pp 645-650.

138. Lei, H. P.; Chen, J.; Ruterana, P., Influences of the biaxial strain and c-screw dislocation on the clustering in InGaN alloys. Journal of Applied Physics 2010, 108 (10).

139. F. A. Ponce, S. S., R. Liu and J. Mei, Lattice mismatch and misfit dislocations in hexagonal GaN-based heterostructures for solid state lighting applications. Acta Microscopica 2007, $16(1-2), 2$.

140. Mei, J.; Liu, R.; Ponce, F. A.; Omiya, H.; Mukai, T., Basal-plane slip in InGaN/GaN heterostructures in the presence of threading dislocations. Applied Physics Letters 2007, $90(17), 171922$.

141. Liu, R.; Mei, J.; Srinivasan, S.; Omiya, H.; Ponce, F. A.; Cherns, D.; Narukawa, Y.; Mukai, T., Misfit dislocation generation in $\mathrm{InGaN}$ epilayers on free-standing GaN. 
Japanese Journal of Applied Physics Part 2-Letters \& Express Letters 2006, 45 (20-23), L549-L551.

142. Tao Tao, Z. Z., Liu Lian, Su Hui, Xie Zili, Zhang Rong, Liu Bin. Xiu Xiangqian. Li Yi, Han Ping, Shi Yi and Zheng Youdou, Surface morphology and composition studies in InGaN/GaN film grown by MOCVD. Journal of Semiconductors 2011, 32 (8), 4.

143. Abell, J.; Moustakas, T. D., The role of dislocations as nonradiative recombination centers in InGaN quantum wells. Applied Physics Letters 2008, 92 (9).

144. Kim, Y. W.; Suh, E. K.; Lee, H. J., Dislocation behavior in InGaN/GaN multiquantum-well structure grown by metalorganic chemical vapor deposition. Applied Physics Letters 2002, 80 (21), 3949-3951.

145. Lei, H.; Chen, J.; Ruterana, P., Role of c-screw dislocations on indium segregation in InGaN and InAlN alloys. Applied Physics Letters 2010, 96 (16).

146. Vanishing critical thickness in asymmetric ferroelectric tunnel junctions: First principle simulations. Journal of Applied Physics 2011, 109 (2), 024103.

147. Shi, L.; Poweleit, C. D.; Ponce, F. A.; Menendez, J.; Chow, W. W., Anisotropic diffusion and drift of photogenerated carriers near coreless dislocations in InGaN quantum well. Applied Physics Letters 2001, 79 (1), 75-77.

148. Keller, S.; Parish, G.; Speck, J. S.; DenBaars, S. P.; Mishra, U. K., Dislocation reduction in GaN films through selective island growth of InGaN. AIP: 2000; Vol. 77, p $2665-2667$. 
149. Seifert, W.; Carlsson, N.; Miller, M.; Pistol, M. E.; Samuelson, L.; Wallenberg, L. R., In-situ growth of quantum dot structures by the Stranski-Krastanow growth mode. Prog. Cryst. Growth Charact. Mater. 1996, 33 (4), 423-471.

150. (a) Cho, H. K.; Lee, J. Y.; Sharma, N.; Humphreys, C. J.; Yang, G. M.; Kim, C. S.; Song, J. H.; Yu, P. W., Effect of growth interruptions on the light emission and indium clustering of InGaN/GaN multiple quantum wells. Applied Physics Letters 2001, 79 (16), 2594-2596; (b) Moon, Y.-T.; Kim, D.-J.; Song, K.-M.; Choi, C.-J.; Han, S.-H.; Seong, T.-Y.; Park, S.-J., Effects of thermal and hydrogen treatment on indium segregation in InGaN/GaN multiple quantum wells. AIP: 2001; Vol. 89, p 6514-6518.

151. Singh, R.; Doppalapudi, D.; Moustakas, T. D.; Romano, L. T., Phase separation in InGaN thick films and formation of $\operatorname{InGaN} / \mathrm{GaN}$ double heterostructures in the entire alloy composition. Applied Physics Letters 1997, 70 (9), 1089-1091.

152. Schulze, F.; Dadgar, A.; Blasing, J.; Diez, A.; Krost, A., Metalorganic vapor phase epitaxy grown InGaN/GaN light-emitting diodes on $\mathrm{Si}(001)$ substrate. Applied Physics Letters 2006, 88 (12).

153. Song, T. L.; Chua, S. J.; Fitzgerald, E. A.; Chen, P.; Tripathy, S., Strain relaxation in graded InGaN/GaN epilayers grown on sapphire. AIP: 2003; Vol. 83, p $1545-1547$

154. Karpov, S. Y., MRS Internet Journal of Nitride Semiconductor Research 1998, 3. 155. Guo, Y.; Liu, X. L.; Song, H. P.; Yang, A. L.; Xu, X. Q.; Zheng, G. L.; Wei, H. Y.; Yang, S. Y.; Zhu, Q. S.; Wang, Z. G., A study of indium incorporation in In-rich InGaN grown by MOVPE. Applied Surface Science 2010, $256(10), 3352-3356$. 
156. Li, N.; Wang, S. J.; Park, E. H.; Feng, Z. C.; Tsai, H. L.; Yang, J. R.; Ferguson, I., Suppression of phase separation in InGaN layers grown on lattice-matched $\mathrm{ZnO}$ substrates. Journal of Crystal Growth 2009, 311 (22), 4628-4631.

157. Pantha, B. N.; Li, J.; Lin, J. Y.; Jiang, H. X., Single phase $\operatorname{In}(x) \mathrm{Ga}(1-\mathrm{x}) \mathrm{N}(0.25<=$ $\mathrm{x}<=0.63$ ) alloys synthesized by metal organic chemical vapor deposition. Applied Physics Letters 2008, 93 (18).

158. Skold, N.; Wagner, J. B.; Karlsson, G.; Hernan, T.; Seifert, W.; Pistol, M.-E.; Samuelson, L., Phase segregation in AllnP shells on GaAs nanowires. Nano Letters 2006, $6(12), 2743-2747$.

159. Kumar, V.; Kr. Sharma, S.; Sharma, T. P.; Singh, V., Band gap determination in thick films from reflectance measurements. Optical Materials 1999, 12 (1), 115-119.

160. Mihopoulos, T. G. MIT, 1997.

161. Schubert, E. F.; Kim, J. K., Solid-State Light Sources Getting Smart. Science 2005, 308 (5726), 1274-1278.

162. Kung P; Razeghi M, III - Nitride wide bandgap semiconductors : a survey of the current status and future trends of the material and device technology. Opto-Electronics Review 2000, 8 (3).

163. S, W. R.; C, E. W., Appl. Phys. Lett. 1964, 4 (5), 89.

164. Duan, X.; Lieber, C. M., Laser-Assisted Catalytic Growth of Single Crystal GaN Nanowires. Journal of the American Chemical Society 1999, 122 (1), 188-189.

165. Kuykendall, T.; Pauzauskie, P. J.; Zhang, Y.; Goldberger, J.; Sirbuly, D.; Denlinger, J.; Yang, P., Crystallographic alignment of high-density gallium nitride nanowire arrays. Nat Mater 2004, 3 (8), 524-528. 
166. Lin, C.; Yu, G.; Wang, X.; Cao, M.; Lu, H.; Gong, H.; Qi, M.; Li, A., CatalystFree Growth of Well Vertically Aligned GaN Needlelike Nanowire Array with LowField Electron Emission Properties. The Journal of Physical Chemistry C 2008, 112 (48), 18821-18824.

167. Calarco, R.; Meijers, R. J.; Debnath, R. K.; Stoica, T.; Sutter, E.; Lüth, H., Nucleation and Growth of GaN Nanowires on Si(111) Performed by Molecular Beam Epitaxy. Nano Letters 2007, 7 (8), 2248-2251.

168. Kim, H. M.; Kim, D. S.; Park, Y. S.; Kim, D. Y.; Kang, T. W.; Chung, K. S., Growth of GaN nanorods by a hydride vapor phase epitaxy method. Advanced Materials 2002, $14(13-14), 991-+$.

169. Hersee, S. D.; Sun, X.; Wang, X., The Controlled Growth of GaN Nanowires. Nano Letters 2006, 6 (8), 1808-1811.

170. Gao, L.; Woo, R. L.; Liang, B.; Pozuelo, M.; Prikhodko, S.; Jackson, M.; Goel, N.; Hudait, M. K.; Huffaker, D. L.; Goorsky, M. S.; Kodambaka, S.; Hicks, R. F., SelfCatalyzed Epitaxial Growth of Vertical Indium Phosphide Nanowires on Silicon. Nano Letters 2009, 9 (6), 2223-2228.

171. Woo, R. L.; Gao, L.; Goel, N.; Hudait, M. K.; Wang, K. L.; Kodambaka, S.; Hicks, R. F., Kinetic Control of Self-Catalyzed Indium Phosphide Nanowires, Nanocones, and Nanopillars. Nano Letters 2009, 9 (6), 2207-2211.

172. Novotny, C. J.; Yu, P. K. L., Vertically aligned, catalyst-free InP nanowires grown by metalorganic chemical vapor deposition. AIP: 2005; Vol. 87, p 203111.

173. Li, H.; Chin, A. H.; Sunkara, M. K., Direction-Dependent Homoepitaxial Growth of GaN Nanowires. Advanced Materials 2006, 18 (2), 216-220. 
174. Vaddiraju, S.; Mohite, A.; Chin, A.; Meyyappan, M.; Sumanasekera, G.; Alphenaar, B. W.; Sunkara, M. K., Mechanisms of 1D Crystal Growth in Reactive Vapor Transport: Indium Nitride Nanowires. Nano Letters 2005, 5 (8), 1625-1631.

175. Vaddiraju, S.; Sunkara, M. K.; Chin, A. H.; Ning, C. Z.: Dholakia. G. R.: Meyyappan, M., Synthesis of Group III Antimonide Nanowires. The Journal of Physical Chemistry C 2007, 111 (20), 7339-7347.

176. Pendyala, C.; Vaddiraju, S.; Kim, J. H.; Jacinski, J.; Chen, Z. Q.; Sunkara, M. K., Self-nucleation and growth of group III-antimonide nanowires. Semiconductor Science and Technology 2010, 25 (2).

177. Tanaka, T.; Nakamoto, M.; Oguni, R.; Lee, J.; Hara, S., Measurement of the surface tension of liquid $\mathrm{Ga}, \mathrm{Bi}, \mathrm{Sn}, \mathrm{In}$ and $\mathrm{Pb}$ by the constrained drop method. Zeitschrift Fur Metallkunde 2004, 95 (9), 818-822.

178. Moser, Z.; Gasior, W.; Debski, A., Database of Pb - free soldering materials, surface tension and density, experiment vs. Modeling. Data Science Journal 2005, 4, 195-208.

179. Wagner R S, E. W. C., Jackson K A; M, A. S., J. Appl. Phys. 1964, 35 (10), 2993.

180. Denton, A. R.; Ashcroft, N. W., Vegard's law. Physical Review: A 1991, +3 (6), 3161.

181. LilientalWeber, Z.; Kisielowski, C.; Ruvimov, S.; Chen, Y.; Washburn, J.; Grzegory, I.; Bockowski, M.; Jun, J.; Porowski, S., Structural characterization of bulk GaN crystals grown under high hydrostatic pressure. Journal of Electronic Materials 1996, $25(9), 1545-1550$. 
182. Wernicke, T.; Ploch, S.; Hoffmann, V.; Knauer, A.; Weyers, M.; Kneissl, M., Surface morphology of homoepitaxial GaN grown on non- and semipolar GaN substrates. Physica Status Solidi B-Basic Solid State Physics 2011, 248 (3), 574-577.

183. Ehrlich, G.; Hudda, F. G., Atomic View of Surface Self? Diffusion: Tungsten on Tungsten. AIP: 1966; Vol. 44, p 1039-1049.

184. Schwoebel, R. L.; Shipsey, E. J., Step Motion on Crystal Surfaces. AIP: 1966; Vol. 37, p 3682-3686.

185. Fichthorn, K.; Scheffler, M., Nanophysics: A step up to self-assembly. Nature 2004, 429 (6992), 617-618.

186. Myslivecek, J.; Schelling, C.; Schaffler, F.; Springholz, G.; Smilauer, P.; Krug, J.; Voigtlander, B., On the microscopic origin of the kinetic step bunching instability on vicinal Si(001). Surface Science 2002, 520 (3), 193-206.

187. Shahedipour-Sandvik, F.; Grandusky, J.; Alizadeh, A.; Keimel, C.; Ganti, S. P.; Taylor, S. T.; LeBoeuf, S. F.; Sharma, P., Strain dependent facet stabilization in selective-area heteroepitaxial growth of GaN nanostructures. Applied Physics Letters 2005, $87(23)$.

188. Strittmatter, A.; Northrup, J. E.; Johnson, N. M.; Kisin, M. V.; Spiberg, P.; ElGhoroury, H.; Usikov, A.; Syrkin, A., Semi-polar nitride surfaces and heterostructures. physica status solidi (b) 2010, 248 (3), 561-573.

189. Huda, M. N.; Yan, Y.; Wei, S.-H.; Al-Jassim, M. M., Electronic structure of ZnO:GaN compounds: Asymmetric bandgap engineering. Physical Review B 2008, 78 (19), 195204. 
190. Jensen, L. L.; Muckerman, J. T.; Newton, M. D., First-Principles Studies of the Structural and Electronic Properties of the (Ga1-xZnx)(N1-xOx) Solid Solution Photocatalyst. The Journal of Physical Chemistry C 2008, 112 (9), 3439-3446.

191. Van de Walle, C. G.; McCluskey, M. D.; Master, C. P.; Romano, L. T.; Johnson, N. M., Large and composition-dependent band gap bowing in InxGal-xN alloys. Materials Science and Engineering B 1999, 59 (1-3), 274-278.

192. Veal, T. D.; Piper, L. F. J.; Jollands, S.; Bennett, B. R.; Jefferson, P. H.; Thomas, P. A.; McConville, C. F.; Murdin, B. N.; Buckle, L.; Smith, G. W.; Ashley, T., Band gap reduction in GaNSb alloys due to the anion mismatch. AIP: 2005; Vol. 87, p 132101.

193. Nunna, K.; Iyer, S.; Wu, L.; Li, J.; Bharatan, S.; Wei, X.; Senger, R. T.; Bajaj, K. K., Nitrogen incorporation and optical studies of GaAsSbN/GaAs single quantum well heterostructures. AIP: 2007; Vol. 102, p 053106.

194. Belabbes, A.; Ferhat, M.; Zaoui, A., Giant and composition-dependent optical band gap bowing in dilute GaSbI?xNx alloys. AIP: 2006; Vol. 88, p 152109.

195. Pei, C. W.; Turk, B.; Héroux, J. B.; Wang, W. I., GaN grown by molecular beam epitaxy with antimony as surfactant. AVS: 2001; Vol. 19, p 1426-1428.

196. Zhang, L.; Tang, H. F.; Schieke, J.; Mavrikakis, M.; Kuech, T. F., The addition of $\mathrm{Sb}$ as a surfactant to $\mathrm{GaN}$ growth by metal organic vapor phase epitaxy. Journal of Applied Physics 2002, 92 (5), 2304-2309.

197. Sheetz, R. M.; Richter, E.; Andriotis, A. N.; Lisenkov, S.; Pendyala, C.; Sunkara, M. K.; Menon, M., Visible-light absorption and large band-gap bowing of GaN(1-x)Sb(x) from first principles. Physical Review B 2011, 84 (7). 
198. Segall, M. D.; Lindan, P. J. D.; Probert, M. J.; Pickard, C. J.; Hasnip, P. J.; Clark, S. J.; Payne, M. C., First-principles simulation: ideas, illustrations and the CASTEP code. Journal of Physics-Condensed Matter 2002, 14 (11), 2717-2744.

199. Vanderbilt, D., SOFT SELF-CONSISTENT PSEUDOPOTENTIALS IN A Generalized EIGENVALUe FORMALISM. Physical Review B 1990, tl (11). $7892-7895$.

200. Giannozzi, P.; Baroni, S.; Bonini, N.; Calandra, M.; Car, R.; Cavazzoni, C.; Ceresoli, D.; Chiarotti, G. L.; Cococcioni, M.; Dabo, I.; Dal Corso, A.; de Gironcoli, S.: Fabris, S.; Fratesi, G.; Gebauer, R.; Gerstmann, U.; Gougoussis, C.; Kokalj, A.; Lazzeri. M.; Martin-Samos, L.; Marzari, N.; Mauri, F.; Mazzarello, R.; Paolini, S.; Pasquarello, A.; Paulatto, L.; Sbraccia, C.; Scandolo, S.; Sclauzero, G.; Seitsonen, A. P.; Smogunov, A.; Umari, P.; Wentzcovitch, R. M., QUUANTUM ESPRESSO: a modular and opensource software project for quantum simulations of materials. Journal of PhysicsCondensed Matter 2009, 21 (39).

201. Berendsen, H. J. C.; Postma, J. P. M.; Vangunsteren, W. F.; Dinola, A.; Haak, J. R., MOLECULAR-DYNAMICS WITH COUPLING TO AN EXTERNAL BATH. Journal of Chemical Physics 1984, 81 (8), 3684-3690.

202. Buckle, L.; Bennett, B. R.; Jollands, S.; Veal, T. D.; Wilson, N. R.; Murdin, B. N.; McConville, C. F.; Ashley, T., Growth of dilute GaNSb by plasma-assisted MBE. Journal of Crystal Growth 2005, 278 (1-4), 188-192.

203. Mondal, A.; Das, T. D.; Halder, N.; Dhar, S.; Kumar, J., Growth of dilute GaSbN layers by liquid-phase epitaxy. Journal of Crystal Growth 2006, 297 (1), 4-6. 
204. Wu, J.; Shan, W.; Walukiewicz, W., Band anticrossing in highly mismatched IIIV semiconductor alloys. Semiconductor Science and Technology 2002, 17 (8), 860-869.

205. Shan, W.; Walukiewicz, W.; Yu, K. M.; Wu, J.; Ager, J. W.; Haller, E. E.; Xin, H. P.; Tu, C. W., Nature of the fundamental band gap in GaNxP1-x alloys. Applied Physics Letters 2000, 76 (22), 3251-3253. 


\section{CURRICULUM VITAE}

\section{Education}

Doctor of Philosophy (Chemical Engineering)

University of Louisville, Louisville, KY.

Master of Science (Chemical Engineering),

Cleveland State University, Cleveland, $\mathrm{OH}$.

Bachelor of Technology (Chemical Engineering),

G. V. P College of Engineering, Visakhapatnam, INDIA.

\section{Professional experience}

Dr. Reddy's Laboratories (Bulk actives unit V, Srikakulam, India): Training in the operation of pharmaceutical bulk drug manufacturing unit (operation of stainless steel and glass lined reactor and clean room crystallization unit)

Reliance Industries Ltd (Jamnagar Refinery, Jamnagar): Training in the operational units and processes in the world's largest grassroots oil refinery.

Indian Petrochemical Corporation ltd (IPCL, Baroda): Training in the Linear Alkyl Benzene (LAB) manufacturing unit.

\section{Publications}

Composition controlled synthesis of $\operatorname{In}_{\mathrm{x}} \mathrm{Ga}_{1-\mathrm{x}} \mathrm{N}$ alloys on $\mathrm{GaN}$ nanowire substrates, C. Pendyala, J. H. Kim, J. B. Jacinski and M. K. Sunkara, (Under Review, Nano Letters).

New materials search for solar hydrogen using nanowires, 14 February 2011, SPIE Newsroom.

Inorganic nanowires: a perspective about their role in energy conversion and storage applications, M.K. Sunkara, C. Pendyala, D. Cummins, P. Meduri, V. Kumar, H.B. Russell, E. L. Clark, and J-H. Kim, invited article, Journal of Physics D: Applied Physics, 44, 174032 (2011) 
A post-synthesis decomposition strategy for Group III-nitride quantum wires, L. Brockway, C. Pendyala, M. K. Sunkara and S. Vaddiraju, Crystal growth and design, 11 (10), 4559-4564 (2011)

Visible light absorption and large band gap bowing in dilute alloys of gallium nitride with antimony, R. M. Sheetz, E. Richter, A. N. Androitis, C. Pendyala, M. K. Sunkara and M. Menon, Physical Review B 84, 075304 (2011)

Self-nucleation and growth of group III antimonide nanowires, C. Pendyala, S. Vaddiraju, J. H. Kim, J. B. Jacinski, Z. Chen and M. K. Sunkara, "', Semiconductor Science and Technology, 25, 024014 (2010), invited article for special issue on nanowires

Hybrid Tin Oxide Nanowires as Stable and High Capacity Anodes for Li-ion Batteries, P. Meduri, C. Pendyala, V. Kumar, G. U. Sumanasekera, and M. K. Sunkara, Nano letters, $9(2), 612(2009)$.

Gas-Phase, Bulk Production of Metal Oxide Nanowires and Nanoparticles Using a Microwave Plasma Jet Reactor, V. Kumar, J. H. Kim, C. Pendyala, B. Chernomordik and M. K. Sunkara, Journal of Physical Chemistry C, 17750 (2008)

Electrochemical pinning of the Fermi level: mediation of photoluminescence from gallium nitride and zinc oxide, V. Chakrapani, C. Pendyala, K. Kash, A. B. Anderson, M. K. Sunkara and J. C. Angus, Journal of the American Chemical Society, 130(39), 12944 (2008).

\section{Presentations}

Poster presentation "Composition controlled synthesis and growth mechanism of ternary $\mathrm{In}_{\mathrm{x}} \mathrm{Ga}_{1-\mathrm{x}} \mathrm{N}$ alloys on GaN nanowires" Gordon Research Seminar on Renewable Energy Solar Fuels, 2011.

Oral presentation "Composition controlled synthesis and growth mechanism of InGaN alloys on GaN nanowire substrates" AIChE annual meeting, 2010.

Poster presentation on "Composition controlled synthesis and growth mechanism of ternary $\operatorname{In}_{x} \mathrm{Ga}_{1-x} \mathrm{~N}$ alloys on GaN nanowires", at International workshop on Nitride Semiconductors 2010 .

Poster presentation on "Theoretical estimation of the electronic structure and properties of $\mathrm{GaSb}_{\mathrm{x}} \mathrm{N}_{1-\mathrm{x}}$ alloys", at $15^{\text {th }}$ annual KY EPSCoR conference, 2010.

Oral presentation on "Controlling the Nucleation and Growth of III-V Semiconductor Nanowires", at AIChE annual meeting 2009.

Oral presentation on "Self-Catalysis Schemes for Group III-Antimonide Nanowires: Antimony Versus Group III Metal", at AIChE annual meeting 2008.

\section{Membership/Affiliations}

Student member of AIChE and MRS 